Science Synthesis, Analysis and Research Program

Earth Monitoring, Analysis, and Prediction (EarthMAP) - A Road Map for Future Integrated Predictive Science at the U.S. Geological Survey

Open-File Report 2021-1108

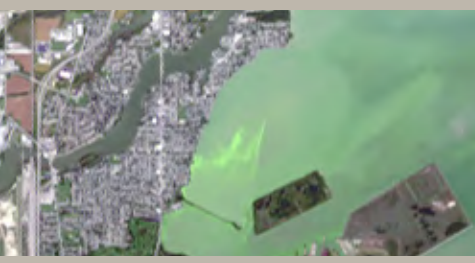

U.S. Department of the Interior U.S. Geological Survey
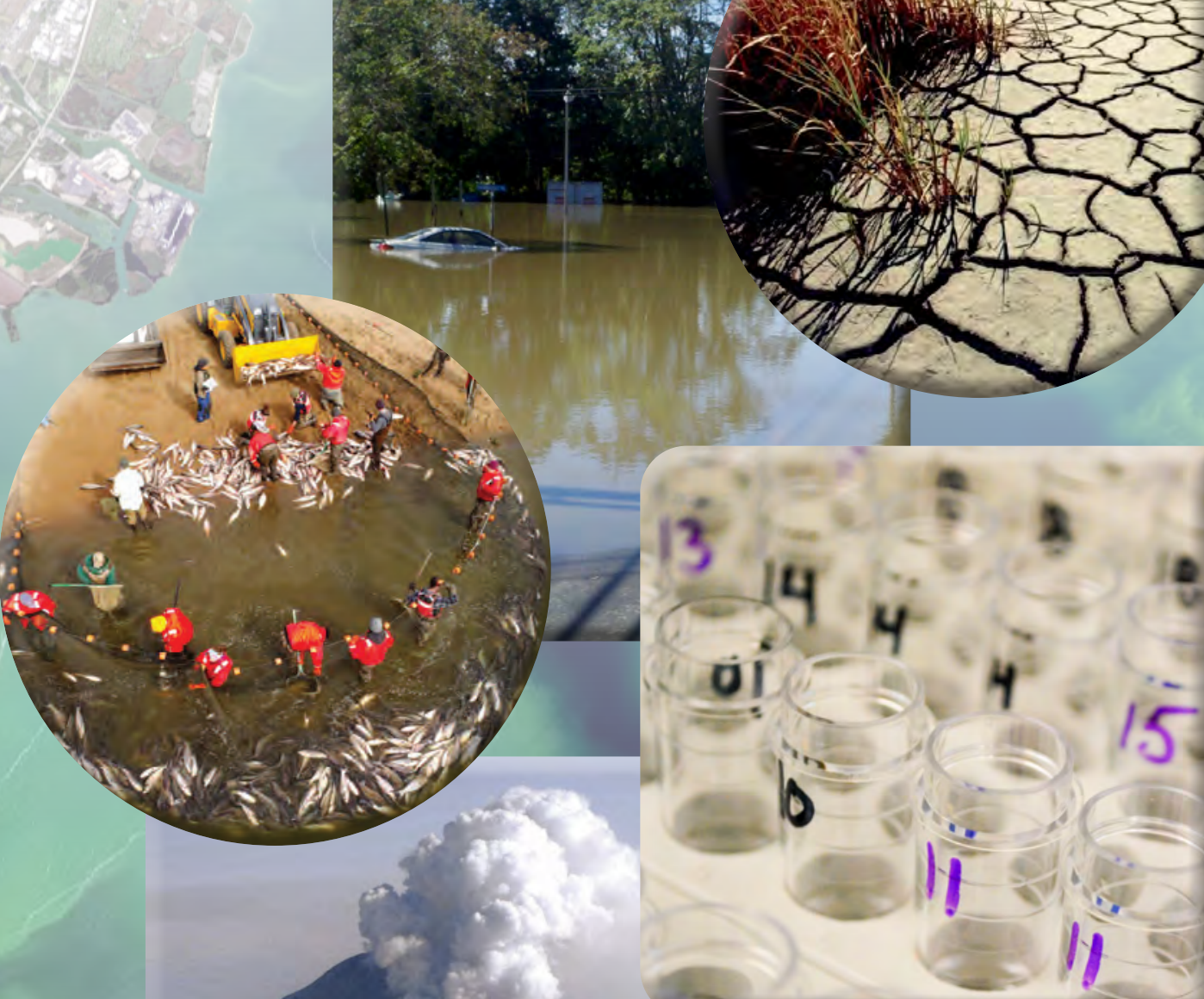

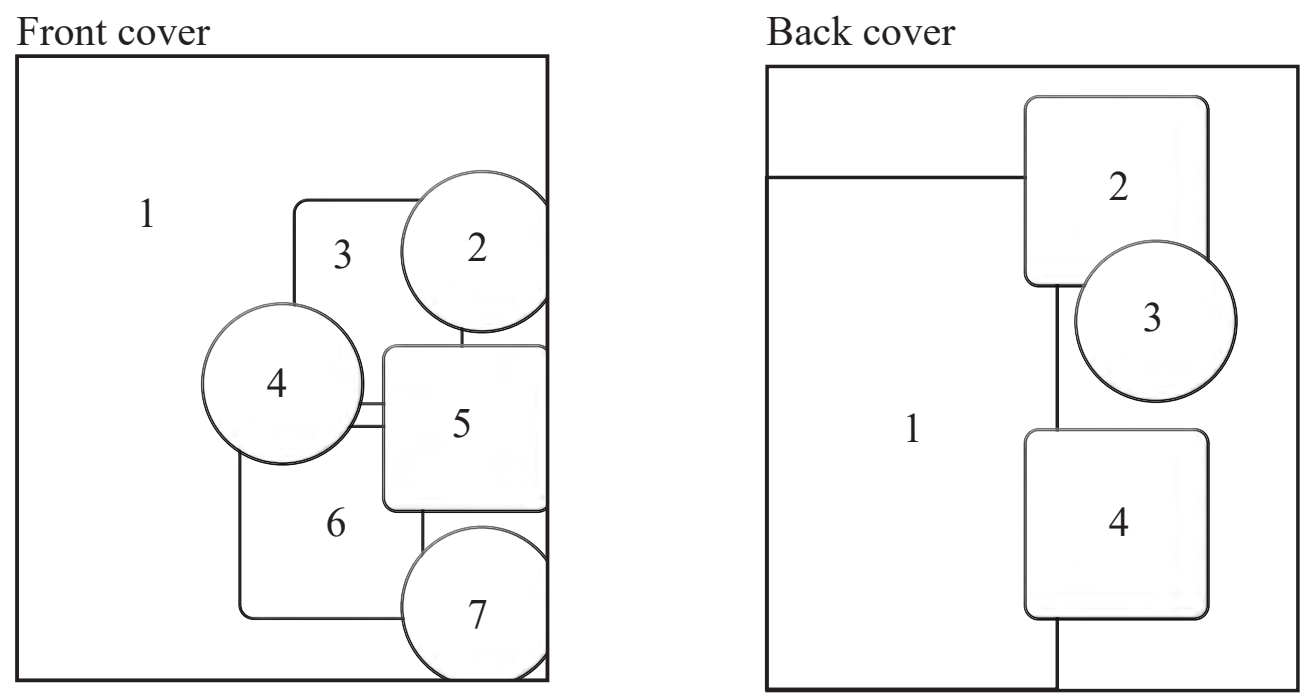

\section{Cover photographs:}

(Front) 1. Satellite imagery showing Lake Erie during a harmful algal bloom (HAB). Photograph from U.S. Geological Survey Multimedia Gallery, November 5, 2019. 2. Drought, September 27, 2001. U.S. Geological Survey photograph. 3. Road ending in submerged car in Bound Brook New Jersey. U.S. Geological Survey photograph, September 2011. 4. Driving invasive carp from a network of nets, U.S. Geological Survey photograph, April 12, 2021. 5. Researchers from the Maryland Department of Natural Resources (DNR) taking water-quality measurements, including dissolved oxygen, aboard the R/V Rachel Carson in the main stem of the Chesapeake Bay. Photograph by Matt Rath, U.S. Geological Survey Chesapeake Bay Program, August 23, 2010. 6. Volcano slider. Photograph from the U.S. Geological Survey Multimedia Gallery. 7. Otter displaying the next generation wildlife tracking device that is affixed to its paw. Photograph by Nicole LaRoche, U.S. Geological Survey.

(Back) 1. Glen Canyon Dam on the Colorado River in Arizona. Photograph by Anne Phillips, U.S. Geological Survey, 2008. 2. Wildland Fire Safety. U.S. Geological Survey Multimedia Gallery, July 9, 2021. 3. Surface-water discharge measuring. U.S. Geological Survey photograph, August 20, 2020. 4. National Water Quality Lab. U.S. Geological Survey Multimedia Gallery, March 30, 2020. 


\section{Use Case Development for Earth Monitoring, Analysis, and Prediction (EarthMAP)_A Road Map for Future Integrated Predictive Science at the U.S. Geological Survey}

By Tamara S. Wilson, Mark T. Wiltermuth, Karen E. Jenni, Robert J. Horton, Randall J. Hunt, Dee M. Williams, Vivian P. Nolan, Nicholas G. Aumen, David S. Brown, Kyle W. Blasch, and Peter S. Murdoch

Science Synthesis, Analysis and Research Program

Open-File Report 2021-1108 


\section{U.S. Geological Survey, Reston, Virginia: 2022}

For more information on the USGS - the Federal source for science about the Earth, its natural and living resources, natural hazards, and the environment—visit https://www.usgs.gov or call 1-888-ASK-USGS.

For an overview of USGS information products, including maps, imagery, and publications, visit https://store.usgs.gov/.

Any use of trade, firm, or product names is for descriptive purposes only and does not imply endorsement by the U.S. Government.

Although this information product, for the most part, is in the public domain, it also may contain copyrighted materials as noted in the text. Permission to reproduce copyrighted items must be secured from the copyright owner.

Suggested citation:

Wilson, T.S., Wiltermuth, M.T., Jenni, K.E., Horton, R.J., Hunt, R.J., Williams, D.M., Nolan, V.P., Aumen, N.G., Brown, D.S., Blasch, K.W., and Murdoch, P.S., 2022, Use case development for earth monitoring, analysis, and prediction (EarthMAP) — A road map for future integrated predictive science at the U.S. Geological Survey: U.S. Geological Survey Open-File Report 2021-1108, 137 p., https://doi.org/10.3133/ofr20211108.

ISSN 2331-1258 (online) 


\section{Contents}

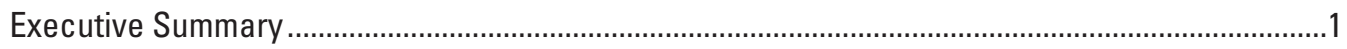

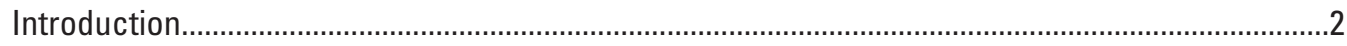

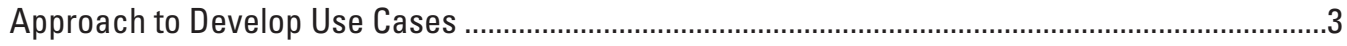

Use Case Development..................................................................................................

Consistency in Use Case Submissions from Scientists across the Nation .......................4

Regional Approaches to Use Case Submissions …….......................................................

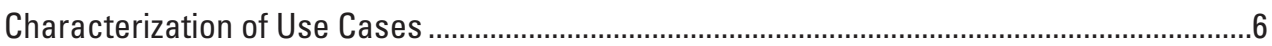

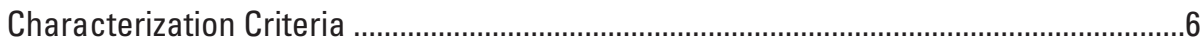

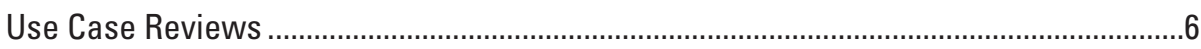

Characterizing Use Cases for Decision Support ............................................................

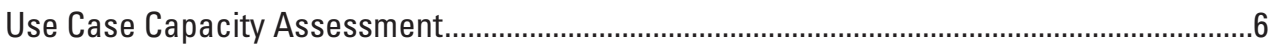

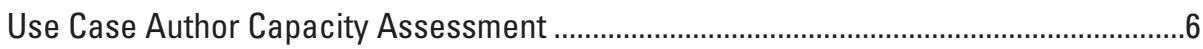

Gap Analysis of Use Cases and Bureau-wide Capacity Assessment ................................7

Common Themes ..........................................................................................................

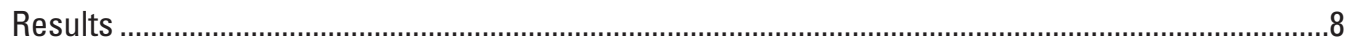

Representation of Use Case Submissions..........................................................................

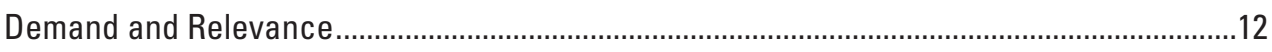

Specificity of the Identified Decisions and Decision Makers ........................................12

Common Types of Decisions Supported ....................................................................12

Opportunities for Additional Integration and Collaboration from the Specific Use Cases Submitted .........................................................................................12

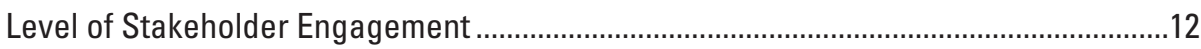

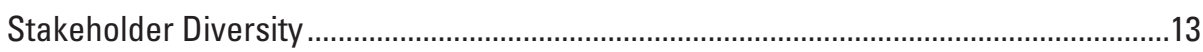

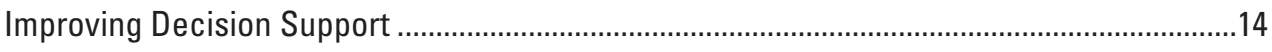

Nature of Decision Support Provided .......................................................................14

Science Capabilities and Advancement Opportunities..........................................................14

Use Case Author Capacity Assessment ........................................................................14

Science and Technology Capacity Gap Analysis ..............................................................14

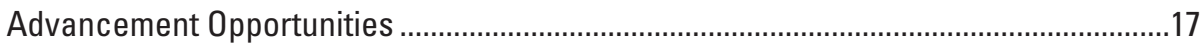

Operational Implementation and Technical Considerations ..................................................17

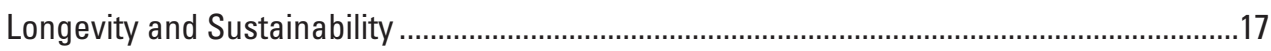

Geographic and Scientific Convergences........................................................................19

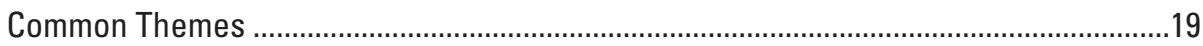

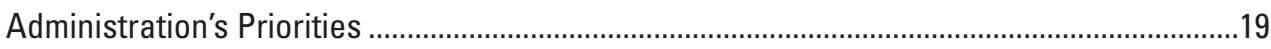

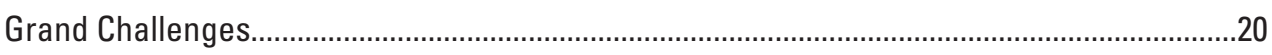

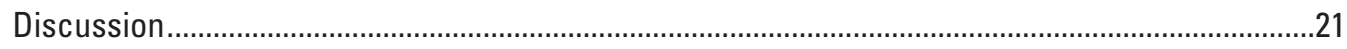

Recommendations for Improving the Use Case Solicitation Process ....................................21

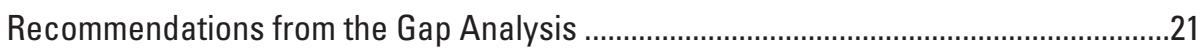

Potential Collaborative Opportunities ........................................................................22

Recommendations from Characterizing Use Cases Through the Lens of

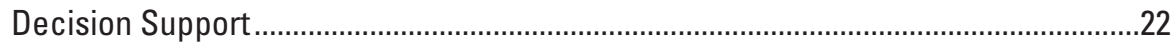

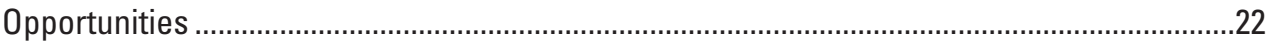

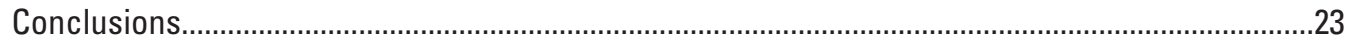

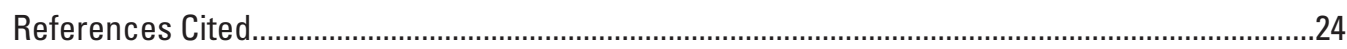


Appendix 1. Earth Monitoring, Analysis, and Prediction (EarthMAP) Use Case

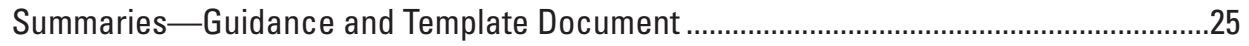

Appendix 2. Use Case Development by Region .....................................................................

Appendix 3. EarthMAP Use Case Characterization Form..........................................................31

Appendix 4. Use Case Author Capacity Assessment Survey ………...........................................34

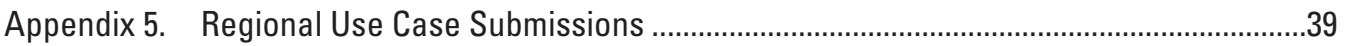

Appendix 6. Use Case Decision Support Statements ..............................................................130

\section{Figures}

1. Pie chart showing number of Use Case submissions by U.S. Geological Survey Regions

2. Graph showing Use Case implementation potential across U.S. Geological Survey Regions

3. Graph showing U.S. Geological Survey Mission Areas represented in Use Cases........10

4. Conceptual illustration of Earth system characterization themes .................................11

5. Graph showing characterization of the 36 Use Cases in terms of the topic type of decision support provided.

6. Graph showing stakeholder engagement by type of activity and level specified within Use Case summary...

7. Graph showing stakeholder diversity expressed by number of Use Cases having different stakeholder categories.....

8. Graph showing distribution of number of stakeholder categories for submitted Use Cases.

9. Graph showing Use Case author assessment of whether current known capacity was sufficient for data and information integration capabilities

10. Graph showing Use Case author assessment of whether current known capacity was sufficient for applicable modeling and prediction capabilities.

11. Graphs showing percent of Capacity Assessment Team Survey responses indicating some level of experience averaged for capabilities within groupings by the three EarthMAP components and percentage of Use Case authors indicating known capacity is insufficient to implement their Use Case.

12. Image showing summary of terms related to capacities for Use Case Predictive Science identified by Use Case authors

13. Image showing first-order keywords and phrases, shown as a word cloud ..................20

14. Pie chart showing common primary objective motivators based on data or information to be developed for submitted Use Cases 


\section{Tables}

1. Approaches to Use Case development by the U.S. Geological Survey Region, number of Use Cases and their application across regions and mission areas represented by the Use Cases received.......................................................................5

2. Metrics used to characterize the specificity with which the target decisions and decision makers for each Use Case were described. (1)

3. Metrics used to characterize the nature of the decision support provided by a

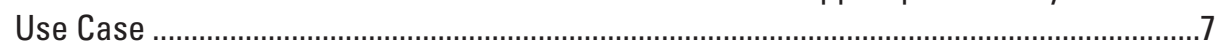

4. Resources available to inform science and technology gap analysis .............................8

5. Top five keywords or phrases identified across Use Cases...............................................

6. Use Case Titles by U.S. Geological Survey Region ............................................................9

7. Listing of data and information capabilities with Use Case Author Capacity Assessment survey results and Capacity Assessment Team's Bureau-wide survey.....18

\section{Conversion Factors}

U.S. customary units to International System of Units

\begin{tabular}{lcl}
\hline \multicolumn{1}{c}{ Multiply } & By & \multicolumn{1}{c}{ To obtain } \\
\hline inch (in.) & \multicolumn{1}{c}{ Length } & \\
inch (in.) & 2.54 & centimeter $(\mathrm{cm})$ \\
foot (ft) & 25.4 & millimeter $(\mathrm{mm})$ \\
mile (mi) & 0.3048 & meter (m) \\
\hline & 1.609 & kilometer $(\mathrm{km})$ \\
\hline acre & Area & \\
acre & 4,047 & square meter $\left(\mathrm{m}^{2}\right)$ \\
acre & 0.4047 & hectare (ha) \\
acre & 0.4047 & square hectometer $\left(\mathrm{hm}^{2}\right)$ \\
square mile $\left(\mathrm{mi}^{2}\right)$ & 259.0 & square kilometer $\left(\mathrm{km}^{2}\right)$ \\
square mile $\left(\mathrm{mi}^{2}\right)$ & 2.590 & hectare (ha) \\
\hline
\end{tabular}




\section{Abbreviations}

$\begin{array}{ll}\text { Al/ML } & \text { Artificial Intelligence/Machine Learning } \\ \text { AK } & \text { USGS Alaska Region } \\ \text { AOOS } & \text { Alaska Ocean Observing System } \\ \text { BLM } & \text { U.S. Bureau of Land Management } \\ \text { CAT } & \text { EarthMAP Capacity Assessment Team }\end{array}$

COVID-19 Novel coronavirus disease-2019 (SARS-CoV-2; severe acute respiratory syndrome coronavirus 2)

CHS Cloud Hosting Solutions Team

CRB Colorado River Basin

CTDEEP Connecticut Department of Energy and Environmental Protection

DEO Department of Environmental Quality

DNA deoxyribonucleic acid

DNR Department of Natural Resources

DOD U.S. Department of Defense

DOI U.S. Department of the Interior

DOT U.S. Department of Transportation

EarthMAP Earth Monitoring, Analysis, and Prediction

eDNA environmental DNA

EPA U.S. Environmental Protection Agency

ERP USGS Energy Resources Program

FEMA Federal Emergency Management Agency

FWS U.S. Fish and Wildlife Service

FY fiscal year

HABMAP Harmful Algal Bloom Monitoring and Prediction

HPC High-Performance Computing

HUD U.S. Housing and Urban Development

IWAA USGS Integrated Water Availability Assessments

LCMAP Land Change Monitoring, Assessment, and Projection

LiDAR Light detection and ranging

LTET Land Treatment Exploration Tool

NASA National Aeronautics and Space Administration

NE USGS Northeast Region

NGO Nongovernmental organization

NGMDB National Geologic Map Database 


$\begin{array}{ll}\text { NGWOS } & \text { USGS Next Generation Water Observing System } \\ \text { NOAA } & \text { National Oceanic and Atmospheric Administration } \\ \text { NPS } & \text { National Park Service } \\ \text { NWIS } & \text { USGS National Water Information System } \\ \text { NWPI } & \text { USGS Northwest-Pacific Islands Region } \\ \text { PMT } & \text { USGS EarthMAP Project Management Team } \\ \text { RM } & \text { USGS Rocky Mountain Region } \\ \text { SE } & \text { USGS Southeast Region } \\ \text { SW } & \text { USGS Southwest Region } \\ \text { UCDT } & \text { EarthMAP Use Case Development Team } \\ \text { UCRB } & \text { Upper Colorado River Basin } \\ \text { USACE } & \text { U.S. Army Corp of Engineers } \\ \text { USDA } & \text { U.S. Department of Agriculture } \\ \text { USFS } & \text { U.S. Forest Service } \\ \text { USFWS } & \text { U.S. Fish and Wildlife Service } \\ \text { USGS } & \text { U.S. Geological Survey } \\ \text { VSC } & \text { USGS Volcano Science Center } \\ \text { WMA } & \text { USGS Water Resources Mission Area }\end{array}$





\title{
Use Case Development for Earth Monitoring, Analysis, and Prediction (EarthMAP)—A Road Map for Future Integrated Predictive Science at the U.S. Geological Survey
}

\author{
By Tamara S. Wilson, Mark T. Wiltermuth, Karen E. Jenni, Robert J. Horton, Randall J. Hunt, Dee M. Williams, \\ Vivian P. Nolan, Nicholas G. Aumen, David S. Brown, Kyle W. Blasch, and Peter S. Murdoch
}

\section{Executive Summary}

The U.S. Geological Survey (USGS) 21st-century science strategy 2020-30 promotes a bureau-wide strategy to develop and deliver an integrated, predictive science capability that works at the scales and timelines needed to inform societally relevant resource management and protection and public safety and environmental health decisions (U.S. Geological Survey, 2021). This is the overarching goal of the USGS Earth Monitoring, Analysis, and Prediction (EarthMAP) vision, which consists of three components: (1) integrated data and information, (2) integrated predictive science, and (3) actionable information - all designed and delivered to respond to user needs. To launch this vision and help shape the design and implementation of integrated predictive science, the USGS Regional Offices each developed a set of use cases (hereafter Use Cases) - short descriptions of potential science applications that could clearly address high priority decision-making needs of our stakeholders and that align with an integrated science focus. Use Cases are not actionable science planning documents, nor stand-alone scholarly works, but should be considered as innovative, next-generation science ideas that can be considered as potential components of science plans still under development. The goal of Use Case development was to (1) identify and characterize existing USGS scientific capacities and expertise that can support science goals and products, (2) identify opportunities to leverage current capacities for next-generation science, and (3) foster engagement across the entire Bureau to further refine the USGS strategy for EarthMAP and integrated predictive science.

The Use Case development effort documented in this report was coordinated by the Use Case Development Team (UCDT), consisting of representatives from each region. The UCDT undertook five tasks: (1) develop a unified approach to engage bureau scientists consistently across all regions in aspirational thinking about what can be accomplished; (2) work with the regions and their Science Centers to generate an initial set of Use Cases, authored directly by scientists; (3) characterize, summarize, and document the initial set of Use Case submissions from authors to illuminate bureau-level demand for integrated science; (4) compare existing and needed capacities from the Use Case descriptions with preliminary results of the EarthMAP Capacity Assessment (Keisman and others, 2021); and (5) describe lessons learned from the Use Case development process and provide recommendations to inform future efforts to generate integrated science activities. This report outlines the approach the UCDT developed to solicit Use Cases from the regions and summarizes the high-level qualitative findings from this first-round effort.

The UCDT received 36 Use Cases from the regions and identified potential points of convergence and commonalities considered useful in making connections among the participating scientists. The Southwest (SW) Region and the Rocky Mountain (RM) Region asked scientists to give special consideration to Use Cases with applicability to the Colorado River Basin, and seven of the Use Cases specifically named that geographic area as a focus. Coastal hazards and coastal resilience were identified in Use Cases from the Alaska (AK), Northeast (NE), and Southeast (SE) Regions. Aspects of wildfire and post-wildfire response were part of Uses Cases from AK, RM, and SW Regions. The greatest convergence of Use Case themes was related to conservation of public lands and waters, which is a powerful linkage lending strength to future collaborative efforts.

The most common type of stakeholder decisions that would be informed by the Use Case science applications were related to adaptation, mitigation, and response (for example, how to increase the resilience of coastal communities to climate-related stressors and how to prevent or respond to harmful algal blooms). Other common types of decisions included water and land management decisions (including operational water management decisions such as reservoir operations and land use planning in the sagebrush biome), decisions about how to manage and conserve habitats and species, and risk management decisions (such as managing the post-wildfire flood risks). These decision types are not exclusive because many Use Cases cross categories. 
Use Case authors identified existing and needed science and technology capabilities required for Use Case implementation, which were then aligned to capabilities assessed in the EarthMAP Capacity Assessment (Keisman and others, 2021). Strong alignment was found for data and information integration approaches, modeling and prediction approaches, and capabilities related to delivery of actionable information. A majority of Use Cases indicated insufficient current capacity for needed data collection methods, data integration, and modeling and prediction approaches, whereas only 25 percent indicated insufficient capacity for actionable information delivery. Overall, many Use Case capacity demand gaps could potentially be met by existing bureau-wide capacity. In addition, nearly half of the Use Cases could potentially be implemented within 3 years if funding, capabilities, and personnel impediments were removed and science priorities were realigned.

Several challenges emerged during the Use Case development process. The first challenge was developing an approach that was flexible enough to accommodate regional differences in planning and implementation, while also ensuring enough guidance to promote meaningful summary analyses. The UCDT encountered a strong demand for continuous communication and education to improve overall understanding of the integrated predictive science strategy. Another challenge was managing expectations about EarthMAP activities as a design effort that was not aligned to an immediate funding opportunity. Connecting the Use Cases to stakeholder needs without the opportunity for direct stakeholder engagement was also challenging. The last notable challenge was in obtaining consistent interpretation and characterization of the qualitative data housed in the narrative descriptions of Use Cases, written in different styles.

Overall, the 36 Use Cases can serve as components of a road map for advancing integrated monitoring and predictive science throughout the USGS by revealing opportunities to (1) encourage cross-region initiatives that address shared interests in common themes by integrating similar Use Cases and through direct involvement of stakeholders in identifying needs and designing effective responses, (2) leverage the Use Cases to target investments that are aligned with the Bureau and Department of the Interior (DOI) priorities, (3) connect Use Cases and the results of the companion EarthMAP Capacity Assessment (Keisman and others, 2021) to identify potential priorities for capacity building investments, and (4) raise awareness of common integrated and interdisciplinary science interests within and across the regions through Use Case and Capacity Assessment summary outreach activities.

\section{Introduction}

How can U.S. Geological Survey (USGS) science better inform societal responses to 21 st-century challenges? Such a question is fundamental to strategic and innovative science planning and associated discussions with USGS stakeholders, partners, and the public. In 2017, a Grand Challenges workshop convened by senior scientists in the USGS articulated a need for enhanced interdisciplinary predictive capabilities targeting a range of earth-science issues (Jenni and others, 2017). The workshop attendees recognized that many factors were converging, including increasingly available, sophisticated technology for monitoring and understanding the Earth system. The opportunity created by rapidly advancing technology was described in an April 1, 2019, communication to all USGS staff:

"A revolution is underway in ground, air, and space-borne sensors, and we now have access to expanding crowd-sourced data capable of providing essential natural science data at unprecedented spatial and temporal resolutions. On-demand storage, processing hardware, and software are changing the paradigm for scientific computing and analysis, allowing scientists to adapt to the age of Big Data. The historical and contemporary natural science data and targeted research for which we are known will be paired with the ongoing explosion in information technology (IT) capabilities to catalyze new types of analysis and enhance our knowledge" (James F. Reilly, U.S. Geological Survey, unpub. data, 2019).

The 2017 workshop also served as the basis for a science planning effort, spearheaded by USGS senior leadership, to envision new and cutting edge integrated predictive science capabilities. Those capabilities for Earth Monitoring, Analyses, and Predictions (EarthMAP) form the foundation of the current USGS Science Strategy (U.S. Geological Survey, 2021). 
EarthMAP incorporates a forward looking, innovative USGS science portfolio capable of delivering actionable information to our stakeholders through 21st-century approaches in monitoring, analysis, and prediction. EarthMAP is a mechanism the USGS will develop to facilitate and apply integrated, multidisciplinary science and technological advancements to meet decision-maker and stakeholder needs. The importance of interdisciplinary approaches to science has long been recognized. Notable in this approach is the inherent need to revisit how the USGS has traditionally performed its work and to identify and elucidate opportunities to increase integrated science activities across the agency. A primary goal of this revisitation is to overcome existing institutional barriers to integrated science and to develop a collaborative culture where integration is considered in the earliest phases of research formulation and includes stakeholder engagement in the initial scoping of work.

To effect such institutional change, USGS leadership formed the EarthMAP Project Management Team (PMT) to facilitate development of integrated predictive science strategy approaches and development. The PMT undertook a bureau-wide Use Case development activity, with the aim of creating a portfolio of the most promising integrated science topics distilled directly from our scientists in the field. Use Cases - as building blocks for integrated predictive scienceidentify high-priority science applications that advance the three major components of EarthMAP: (1) data collection and information integration, (2) integrated predictive science, and (3) actionable information delivered at the speed and scale of decision making. Use Cases are defined here as descriptions of potential science applications that address high priority decision-making needs relevant to stakeholders and require an integrated science focus. Use Cases are not stand-alone scholarly works, nor are they actionable science planning documents. Use Cases should be considered as innovative, next-generation science ideas that can be considered as potential components of science plans still under development. Overall, Use Cases are driven by the needs of stakeholders and serve to identify existing and future USGS (and partner) science capacity and technological innovations that will meet those needs. By co-developing Use Cases directly with stakeholders, the USGS will be better positioned to deliver science at the speed and scales that those stakeholders need.

The Use Case Development Team (UCDT), composed of personnel nominated by the USGS Regional Directors, was formed to lead the effort to create a set of clear and compelling Use Cases representing the diversity of stakeholder needs, science topics, and expertise across the USGS regions. The charge of the UCDT was to (1) identify and characterize existing USGS scientific capacities and expertise able to support EarthMAP goals and products, (2) identify opportunities to leverage current capacities for next-generation science, and (3) foster engagement across the Bureau to further refine the integrated predictive science concept. The objective was to synthesize potential Use Cases into common themes, capacities, and opportunities, leveraging existing expertise along with next-generation advancements to increase USGS predictive science capacity. The Use Cases were intended to give specificity to high-level concepts through their description of potential USGS work applied to real-world Earth system monitoring, analyses, and prediction challenges and to represent examples of future-looking, aspirational, integrated predictive science. The broad scope of the UCDT effort was "to take stock of where USGS science will go in the next decade to ensure that we respond to 21 st-century challenges with 21 st-century science and technology"

(James F. Reilly, U.S. Geological Survey, unpub. data, 2019).

The purpose of the UCDT's national Use Case development effort was to ensure a level of consistency in communication, information requests, and delivery to facilitate comparison and summary reporting, while also being flexible enough to accommodate regional variation in approach. This report documents (1) regional processes used to solicit Use Cases, (2) the national approach in characterizing the Use Cases, (3) the capacities needed for those Use Cases to move from concept to operation, (4) national and regional Use Case results, (5) lessons learned, and (6) opportunities and next steps. This development effort can serve as a template for future, iterative efforts at science innovation and provide a road map for leveraging cross-regional capacities and collaborative opportunities and more broadly, as outlining potential pathways for long-term programmatic investment.

\section{Approach to Develop Use Cases}

Since fall 2019, the EarthMAP PMT has been communicating an integrated predictive science concept to engage scientists and stakeholders in aspirational thinking about what the USGS can accomplish with a fully realized EarthMAP-type approach. This communication and outreach effort served as the foundation for the Use Case development activities, which were initiated by the UCDT in summer 2020 to solicit Use Case examples that the USGS and our partners and stakeholders might use as planning targets for integrated predictive science. 


\section{Use Case Development}

The primary objective of the UCDT was to identify high-priority national and regional Use Cases that reflect EarthMAP goals, desired outcomes, and products by using a bottom-up approach. An overarching goal emphasized in the Use Case development process was that ideas were founded upon data, analysis, and integrated and predictive science information needs that were previously identified by key local, state, and regional stakeholders who had existing relationships with USGS staff and centers. By building a shared sense of ownership and investment in the future of integrated predictive science and a 21st-century USGS, we attempted to foster employee level buy-in to the organizational concept of integrated predictive science.

\section{Consistency in Use Case Submissions from Scientists across the Nation}

To ensure consistency in the Use Case development process and subsequent evaluation, the UCDT developed a Use Case Summary template, accompanied by associated guidance documentation (appendix 1). The template defined and outlined five key elements for Use Case contributors (that is, USGS scientists) to address in their Use Case submission:

\section{Demand and Relevance}

Use Cases define the question(s) to be addressed, relevance as a societal issue, and who benefits from any proposed science. Contributors described why an EarthMAP approach is necessary to advance and deliver relevant USGS science. Furthermore, Use Cases describe alignment with existing regional and partner or stakeholder priorities, extensibility in geographic scale, topical integration, and applicability for multiple decision-support applications.

\section{Improving Decision Support}

Contributors identified what aspect of the decision process would be targeted for improvement (for example, information accuracy and resolution, information scope and value, timeliness of delivery, accessibility, and useability) and how it fits into existing decision and action frameworks. Use Case summaries included a description of the co-development process with stakeholders and implementation approach of the resulting information product.

3. Science Capabilities and Advancement Opportunities
Use Cases identified and described current USGS expertise and data collection mechanisms, while also defining opportunities for science advancement, new data needs, and integration. These opportunities included interdisciplinary approaches, inter-regional collaboration, as well as improving partnerships with local, state, federal, non-profit, or other entities. Use Cases build upon existing data streams and research programs, while also outlining how the USGS might more accurately and quickly deliver actionable, integrated scientific information through partnership and stakeholder engagement.

4. Operational Implementation and Technical Considerations

Contributors were asked to identify challenges, including gaps in current capabilities, existing bottlenecks of data access, organization, integration, and delivery, as well as to describe implementation challenges given current and potential organizational capacity and partnerships. Opportunities to co-produce knowledge and technological processes through deliberate external partnerships in addressing the Use Case were provided, along with existing and potential partners who could work with the USGS toward solutions for improved decision making. The need for any new metadata standards to support tracking of data provenance and lineage also were identified. Use Case alignment with current or potential computational resources was encouraged to manage data production, processing, and delivery, or identify where partnerships could assist with such efforts.

\section{Longevity and Sustainability}

Use Case summaries address whether the earth science information product(s) have long-term viability and sustained relevance to identified stakeholders and offer opportunities to extend the work in a sustainable way to benefit other stakeholders. Use Cases identified existing and needed requirements to attain long-term viability of the approach. Use Cases also defined the activities to be sustained throughout the life cycle of the decision/action framework as necessary to ensure continuity of decision support. Contributors described the transferability and scalability of deliverables across geographies, disciplines, and other applications. The UCDT developed the Use Case Summary Guidance document (appendix 1) to serve as a screening tool to help regional leaders identify high priority Use Cases and for the UCDT to evaluate and summarize findings across all submissions. 


\section{Regional Approaches to Use Case Submissions}

Region-led efforts in communicating, coordinating, and requesting Use Case submissions for this initial effort varied significantly as regions exercised their existing planning models and brought their current strategic interest and opportunities into play. Some regions held multi-day workshops on the EarthMAP concept, some established working groups, some allowed submissions from all interested scientists, some identified focal science topics and utilized writing teams and workshops, and still others prioritized only achievable and fundable projects. The unifying theme was a bottom-up regional approach to requesting next-generation science ideas from the field, relying on scientists with direct knowledge of stakeholder needs.

To ensure uniform communication to the UDCT, each region assigned a representative to the UCDT (region lead). Appendix 2 contains detailed descriptions of the process and steps taken by each region, which provides a menu of options for future Use Case development or any enterprise-level initiative requiring ground-level input from USGS scientists for the development of next-generation science applications. Table 1 summarizes the processes used in each region.

Table 1. Approaches to Use Case development by the U.S. Geological Survey Region, number of Use Cases and their application across regions and mission areas represented by the Use Cases received.

[EarthMAP, Earth Monitoring, Analysis, and Prediction; UCDT, Use Case Development Team; PI, Principal Investigator]

\begin{tabular}{|c|c|c|}
\hline Use Case development process & $\begin{array}{l}\text { Number of Use Cases } \\
\text { with connections to } \\
\text { other regions }\end{array}$ & $\begin{array}{l}\text { Mission areas } \\
\text { represented } \\
\text { in Use Cases }\end{array}$ \\
\hline \multicolumn{3}{|l|}{ Alaska } \\
\hline $\begin{array}{l}\text { Initial selection by Regional Office and Center Directors based on alignment of exist- } \\
\text { ing regional priorities with EarthMAP; additional Use Cases identified through } \\
\text { meetings of regional management }\end{array}$ & $3($ of 5$)$ & $\begin{array}{l}\text { Core Science Systems, } \\
\text { Ecosystems, Natural } \\
\text { Hazards, Water Resources }\end{array}$ \\
\hline $\begin{array}{l}\text { Region leadership (Director, and Science Coordinators) selected five focal topics, } \\
\text { and then engaged their science leadership through a Science Chief Forum. Science } \\
\text { chiefs refined ideas for Use Case within each focal topic, and the Region Science } \\
\text { Coordinators integrated ideas into five to be developed further for submission to the } \\
\text { UCDT }\end{array}$ & $1($ of 5$)$ & $\begin{array}{l}\text { Core Science Systems, } \\
\text { Ecosystems, Natural } \\
\text { Hazards, Water Resources }\end{array}$ \\
\hline \multicolumn{3}{|l|}{ Northeast } \\
\hline \multicolumn{3}{|l|}{ Northwest-Pacific Islands } \\
\hline $\begin{array}{l}\text { Centers and the Northwest Climate Adaptation Science Center identified capabilities } \\
\text { and potential science topics aligned with EarthMAP. A selected team of mid-career } \\
\text { scientists were charged with developing initial interdisciplinary use cases, all of } \\
\text { which were put forward by regional management }\end{array}$ & 4 (of 5) & $\begin{array}{l}\text { Core Science Systems, } \\
\text { Ecosystems, Energy } \\
\& \text { Minerals, Water } \\
\text { Resources }\end{array}$ \\
\hline \multicolumn{3}{|l|}{ Southeast } \\
\hline $\begin{array}{l}\text { Annual Regional Science Workshop focused on EarthMAP; one activity during that } \\
\text { workshop focused on identifying potential Use Cases }\end{array}$ & 0 (of 2) & All \\
\hline \multicolumn{3}{|l|}{ Southwest } \\
\hline
\end{tabular}




\section{Characterization of Use Cases}

\section{Characterization Criteria}

The UCDT characterized Use Case submissions to provide a basis for communicating to USGS leadership about commonalities across regions, how the science applications aligned to the EarthMAP concept, the types of decisions they supported, and project maturity. Characterization criteria also assist in identifying patterns within the Use Case summaries, potential synergies, opportunities for further refinement, and to determine how the Use Cases could be leveraged to further develop integrated predictive science opportunities. Data summaries from the Use Cases were organized by major criteria representing the theme, scope, readiness, and advancement within each Use Case. The five key elements outlined in the Use Case guidance and template (appendix 1) were used to form an initial 18-point characterization.

\section{Use Case Reviews}

Use Case reviews were iterative and involved three stages. Reviews were initially conducted by two UCDT members as primary reviewers who were from outside the region of submission and tasked with evaluating whether each of the characterization criteria had been addressed. Additional categorical data were collected, including the region, mission area, and scientific discipline, and also the capabilities and capacities planned for use and needed, and the number of identified stakeholders. A second-pass evaluation garnered additional information, which led to the development of a characterization form (appendix 3) to capture information relevant to the PMT and the EarthMAP Executive Oversight Committee. The characterization form has different evaluation categories chosen to provide basic topical information to determine how well each Use Case supported EarthMAP objectives. New information was obtained and summarized on the degree of specificity in the decision being informed, the aspect of decision process being improved, the estimated level of stakeholder engagement, the degree of aspiration or advancement from existing approaches, the degree of specificity in relation to the advancement opportunity, estimated implementation timeline, scalability, degree of feasibility, execution challenges, and major impediments to success. Though based on UCDT professional expertise, much of the characterization is qualitative and subjective. Any major review discrepancies were reconciled by the region lead.

The final review stage involved small group work to better determine the type and level of stakeholder engagement, the issue and decision being informed, project feasibility and maturity, science and technology capacity assessment, and areas of synergy and convergence among Use Cases. Each new information category was assigned a working group of four to five UCDT members to collectively define, evaluate, and summarize the topical category. This approach allowed for more in-depth, discussion-based analyses, improved understanding of the characterization objective, cooperative design in the topical approach, and agreement upon a consistent interpretation of results. This approach also led to more consistent, comparable results and greater confidence in Use Case interpretation and characterization.

\section{Characterizing Use Cases for Decision Support}

The decision-support focus of each Use Case was extracted and summarized by defining the bracketed elements in the following Decision Support Statement:

This Use Case will produce (product or result) that (will/can) be used by (decision-maker[s]) to support (one or more decisions).

Each Use Case was characterized for the specificity and clarity of the defined target decision(s) - that is, the decisions that the science application was intended to support-and in how clearly, they specified the decision makers who would use the science output. The metrics described in table 2 were used in the decision support characterization. The types of decisions supported were also characterized: whether the information was judged to be directly useful for the identified target decisions or whether it was indirectly useful (metrics in table 3).

\section{Use Case Capacity Assessment}

\section{Use Case Author Capacity Assessment}

The UCDT requested completion of a follow-up Use Case Capacity Assessment Survey from the Use Case authors (appendix 4) to assess the capabilities and capacities needed for the proposed science application. The goal of the follow-up survey was to determine linkages and discrepancies between the Use Case Author Capacity Assessment and the EarthMAP Capacity Assessment Team's (CAT) Bureau-wide Capacity Assessment Survey (Keisman and others, 2021). Survey design was simple with consistently formatted responses grouped by EarthMAP Concepts: (1) Data and Information Integration, (2) Predictive Modeling, and (3) Actionable Information, similar to the approach of Keisman and others (2021). For each capability defined in the CAT Bureau-wide Capacity Assessment Survey, the follow-up survey asked Use Case authors to assess needed capacity using one of three response options: (1) existing capacity is sufficient for the requirements of the Use Case, (2) existing capacity is insufficient for the Use Case requirements, and (3) the capacity is not available and would need to be developed. 
Table 2. Metrics used to characterize the specificity with which the target decisions and decision makers for each Use Case were described.

[ALBIRA, Alabama Barrier Island Restoration Assessment]

\begin{tabular}{lr}
\hline \multicolumn{1}{c}{ Specificity of the target decision(s) } & Specificity of the decision makers \\
\hline $\begin{array}{l}1=\text { general or generic } \\
\text { (for example, "Land and resource management decisions") }\end{array}$ & $\begin{array}{r}1=\text { general, generic, or many possible listed } \\
\text { (for example, many Use Cases provide a long list of potential } \\
\text { decision-makers who might use the products) }\end{array}$ \\
\hline $\begin{array}{l}2=\text { general but with at least one specific example } \\
\text { (for example, National Park Service managers, with Indiana Dunes } \\
\text { National Park as the specific decision maker) }\end{array}$ \\
$\begin{array}{ll}\text { (for example, "optimizing reservoir operations, water deliveries, } \\
\text { hydropower production, and other water management objectives") }\end{array}$ & $\begin{array}{c}\text { (for example, "state of Alabama" for the ALBIRA Use Case) } \\
\text { specific }\end{array}$ \\
\hline $4=$ no decision identified & $4=$ no decision maker identified \\
\hline
\end{tabular}

Table 3. Metrics used to characterize the nature of the decision support provided by a Use Case.

\begin{tabular}{|c|c|}
\hline Metric & Nature of the decision support provided \\
\hline 1 & $\begin{array}{l}\text { Direct decision support: delivers actionable information (that is, provides all of the science information necessary to support the } \\
\text { identified decision) }\end{array}$ \\
\hline 3 & $\begin{array}{l}\text { Indirect decisions support: creates science or data that requires further processing, analysis, or combination to create the actionable } \\
\text { information (for example, produces data that will be input for other models) }\end{array}$ \\
\hline 4 & Product not clearly described \\
\hline
\end{tabular}

\section{Gap Analysis of Use Cases and Bureau-wide Capacity Assessment}

A gap analysis was performed to highlight insufficient, yet necessary, capacities for Use Case implementation (table 4) and to inform future capacity investment. The UCDT and CAT convened a joint group to discuss and conduct the gap analysis from the Use Cases to identify capabilities, the Use Case authors' assessment of available capacities, and the CAT Bureau-wide Capacity Assessment Survey results (hereafter CAT Survey). Results of the gap analysis, though limited in scope, provide useful preliminary information. A more thorough gap analysis could incorporate additional information from sources throughout the Bureau (table 4).

The joint CAT and UCDT gap analysis effort can inform USGS leadership on investment opportunities required for science and technology advancement in support of integrated science through EarthMAP or any strategic, next-generation science planning initiative. A consideration when using data from UCDT and CAT surveys for gap analysis is that survey responses originate from different respondent perspectives. The CAT Survey (Keisman and others, 2021) was intended as a broad inventory of our current science capabilities and capacities, without considering next-generation science applications. An additional consideration while interpreting capacity gaps and assessing capacities bureau-wide is the level of awareness and communication among USGS scientists. Future gap analyses would likely benefit from additional information sources throughout the Bureau and a focus on priority integrated science plans with more, explicitly expressed, capacity demand.

\section{Common Themes}

To find common themes within the Use Cases, the UCDT developed a synergy categorization using two distinct set of categories centered around science application objectives. The first, or "primary objective motivator," represent the motivators for why the data or science should be developed and the second, or "secondary objective motivator," focused on the multiple topical areas being addressed. After initial Use Case review, the primary objective motivators were split into five thematic groupings (resource protection, regulatory/legal, hazards, climate change, and research and development). Recognizing that motivator overlap would occur, the primary motivator was identified based on the associated science and decision types. Only one primary motivator was selected and multiple secondary motivators were identified. 
Table 4. Resources available to inform science and technology gap analysis.

[Resources are listed by those that represent existing capacities within USGS and by those that represent future needed capacity to apply integrated science aligned to EarthMAP concepts. The * indicates resources used for our preliminary gap analysis.]

Resources representing existing EarthMAP capacities (supply)

Capacity assessment survey (Keisman and others, 2021)*

Catalogs/databases of USGS data, models, publications, and people

Associate Chief Information Officer (ACIO) advanced Information and

Management Technology (IMT) resources list

Mission area and program-specific requirements

EarthMAP-related work-group results (for example, "Building

EarthMAP Capacity")
Resources representing future EarthMAP needs (demand)

Grand Challenges report (Jenni and others, 2017)*

EarthMAP Use Cases*

EarthMAP requirements

Mission area integrated science priorities (5-year roadmaps)

Needs identified via partner and stakeholder engagement

EarthMAP-related work-group results (for example, Building EarthMAP Capacity)
To identify common and crosscutting topics addressed by the regional Use Cases, first-, second-, and third-order keywords and phrases were identified for each Use Case using word cloud analysis, which is a visual representation of word frequency (Ramsden and Bate, 2008). The UCDT attempted, unsuccessfully, to develop a common set of keywords and phrases based on mission area and individual program titles. Future Use Case evaluations would benefit from a common set of keywords or phrases and criteria to apply them in a consistent manner. Consequently, word cloud analysis was applied to the three sets of keywords/phrases, as well as the entire list of keywords for all three keyword levels (first-, second-, and third-order keywords; table 5).

Table 5. Top five keywords or phrases identified across Use Cases.

\begin{tabular}{|c|c|c|}
\hline $\begin{array}{c}\text { First order } \\
\text { keyword or phrase }\end{array}$ & $\begin{array}{c}\text { Second order } \\
\text { keyword or phrase }\end{array}$ & $\begin{array}{c}\text { Third order keyword } \\
\text { or phrase }\end{array}$ \\
\hline $\begin{array}{l}\text { Resources } \\
\text { management }\end{array}$ & Water quality & Forecasting \\
\hline Water quality & Ecology & Modeling \\
\hline Water availability & $\begin{array}{l}\text { Species distribution } \\
\text { model }\end{array}$ & Decision support \\
\hline Wildfire & Nutrients loading & Data integration \\
\hline Hazard & Data integration & Monitoring \\
\hline
\end{tabular}

\section{Results}

\section{Representation of Use Case Submissions}

A total of 36 Use Cases were submitted (fig. 1; table 6; appendix 5). At least 18 submissions indicated applicability across regions (fig. 2). All mission areas were represented, with some Use Cases crossing multiple mission areas (fig. 3).

Use cases were evaluated in the context of Earth systems science, as described by societal "Grand Challenges" in Jenni and others (2017) that integrates traditional scientific disciplines and the full portfolio of USGS science activities (fig. 4). The majority of Use Cases identified more than five Earth system characterization themes, as detailed in figure 4, including land surface, surface and groundwater, fauna, flora, humans, and geology.

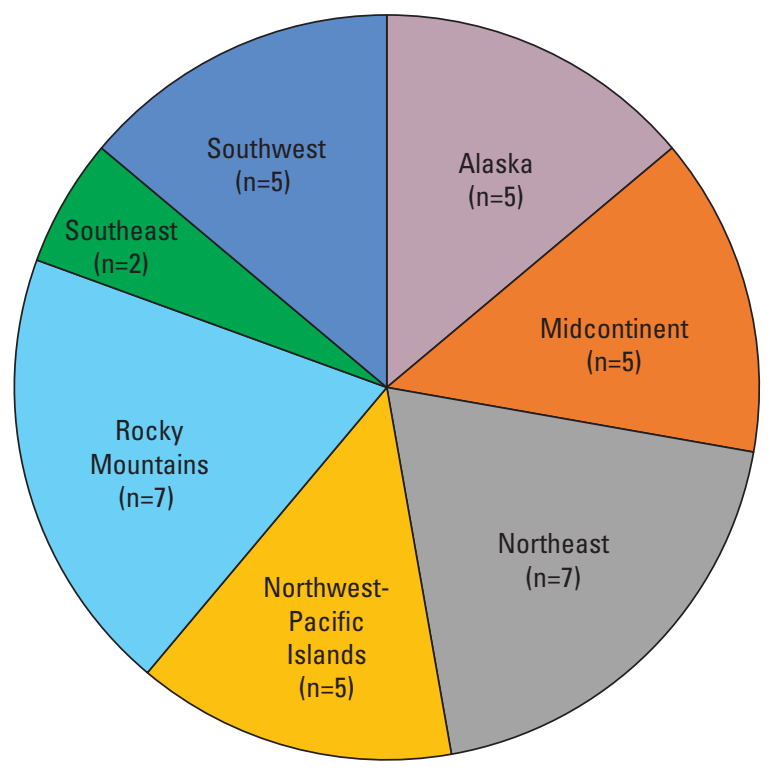

Figure 1. Number of Use Case submissions by U.S. Geological Survey Regions. 
Table 6. Use Case titles by U.S. Geological Survey Region.

\begin{tabular}{|c|c|}
\hline Region & Use Case title \\
\hline Alaska & $\begin{array}{l}\text { - Building an Integrated Coastal Hazard Assessment and Mitigation Strategy with Bering Sea Communities of Alaska } \\
\text { - Customized Arctic Map Products for State, Federal, and International Use } \\
\text { - Enhanced Delivery of Integrated Ecosystem Model Output } \\
\text { - Machine Learning Analysis of Near Real-time Imagery of U.S. Volcanoes } \\
\text { - Wildlife Tracking Portal to Improve Data Documentation, Delivery, and Analysis }\end{array}$ \\
\hline Midcontinent & $\begin{array}{l}\text { - EarthMAP as a strategic, systematic interface between USGS and the National Park Service Resource Stewardship } \\
\text { - Strategies } \\
\text { - U.S. Geological Survey Integrated Ecohydrology Science: A New Path to Advance Water Science, Ecosystem Science, and } \\
\text { Earth-System Prediction } \\
\text { - Harmful Algal Bloom Monitoring and Prediction (HABMAP) } \\
\text { - Legacy Sources of Nutrients and Implications for Management Strategies } \\
\text { - NowCast+: Expanding eDNA tracking of pathogens and invasive species using the NowCast Framework }\end{array}$ \\
\hline Northeast & $\begin{array}{l}\text { - An integrated framework for assessing bird populations to better understand drivers of population change and project future } \\
\text { outcomes } \\
\text { - Collaborative science to enhance resilience in coastal cities, towns, and rural landscapes: The Urban Coastal Resilience } \\
\text { Initiative (UCRI) } \\
\text { - Improving National Fish Habitat Assessments using the NHDPlus HR: An EarthMAP Pilot Effort in the Delaware River } \\
\text { Basin } \\
\text { - Integrated Monitoring, Analysis, and Modeling for Enhanced Predictive Capability of Nutrient Loads from the Connecticut } \\
\text { River Basin to Long Island Sound } \\
\text { - Integrated multidisciplinary science to inform habitat conservation and management efforts for fish and wildlife in the } \\
\text { Delaware Watershed } \\
\text { - Observation, assessment, prediction, and operationalizing of a regional analysis of sediment and co-contaminate flux and } \\
\text { sediment sources in the Delaware River Basin } \\
\text { - Optimization of Mineral Resource Data: Mine Wastes, Resource Assessments, and Focus Areas }\end{array}$ \\
\hline $\begin{array}{l}\text { Northwest } \\
\text { - Pacific } \\
\text { Islands }\end{array}$ & $\begin{array}{l}\text { - From water to wildlife: linking water timing and availability to subalpine and alpine vegetation and wildlife populations } \\
\text { - Geography of change-Synthesizing multiple dimensions of anthropogenic global change with indicators of ecological and } \\
\text { hydrological conditions } \\
\text { - Surface-Water Index of Permanence for Ecohydrology (SWIPE) } \\
\text { - Sustaining the Western Range: Actionable Science for Effective Management of the Sagebrush } \\
\text { - Thunder and lightning - social, ecological, and hydrologic assessment of drought in the Donner und Blitzen River } \\
\text { watershed }\end{array}$ \\
\hline Southeast & $\begin{array}{l}\text { - Alabama Barrier Island Restoration Assessment } \\
\text { - Mississippi Alluvial Plain Water Availability Study }\end{array}$ \\
\hline Southwest & $\begin{array}{l}\text { - A Drought Data Explorer for the Colorado River Basin: Integrated and dynamic web-based delivery of actionable } \\
\text { information } \\
\text { - Data and Modeling Platform to Support Forecasting California Water Project Effects } \\
\text { - Enhancing Situational Awareness and Forecast Models for Post-Wildfire Runoff, Recharge, Water Quality, and } \\
\text { Sedimentation } \\
\text { - Improving Daily Modeled Estimates of High Elevation Snowpack to Boost Runoff Forecast Accuracy in Colorado Basin } \\
\text { Watersheds } \\
\text { - Predicting water quality and environmental health in rivers of the Colorado River Basin affected by wildfire }\end{array}$ \\
\hline
\end{tabular}




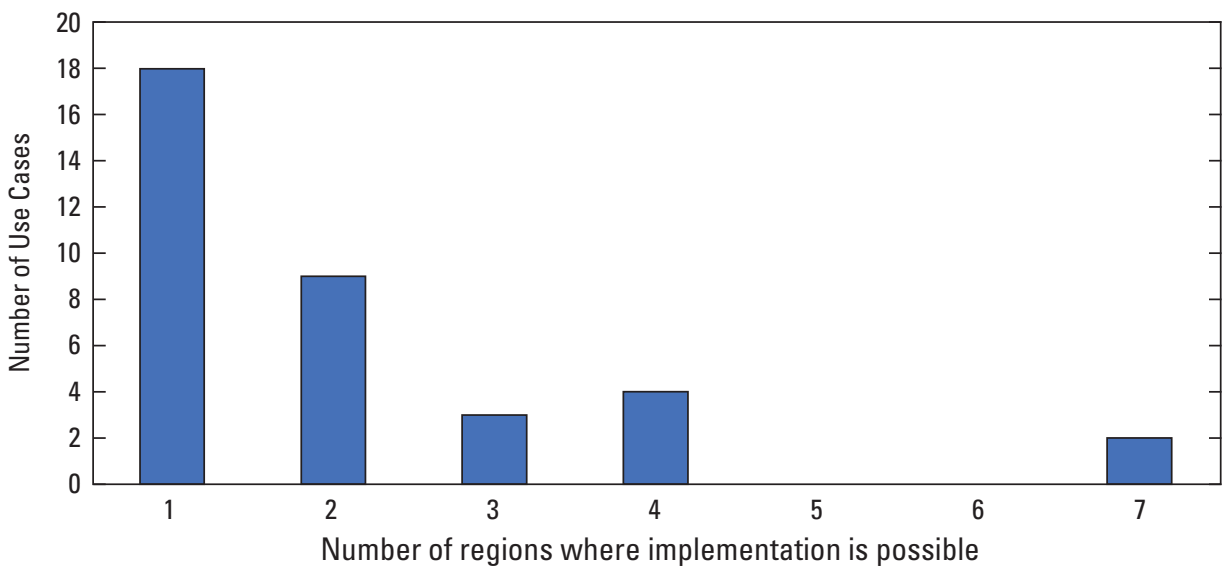

Figure 2. Use Case implementation potential across U.S. Geological Survey Regions.

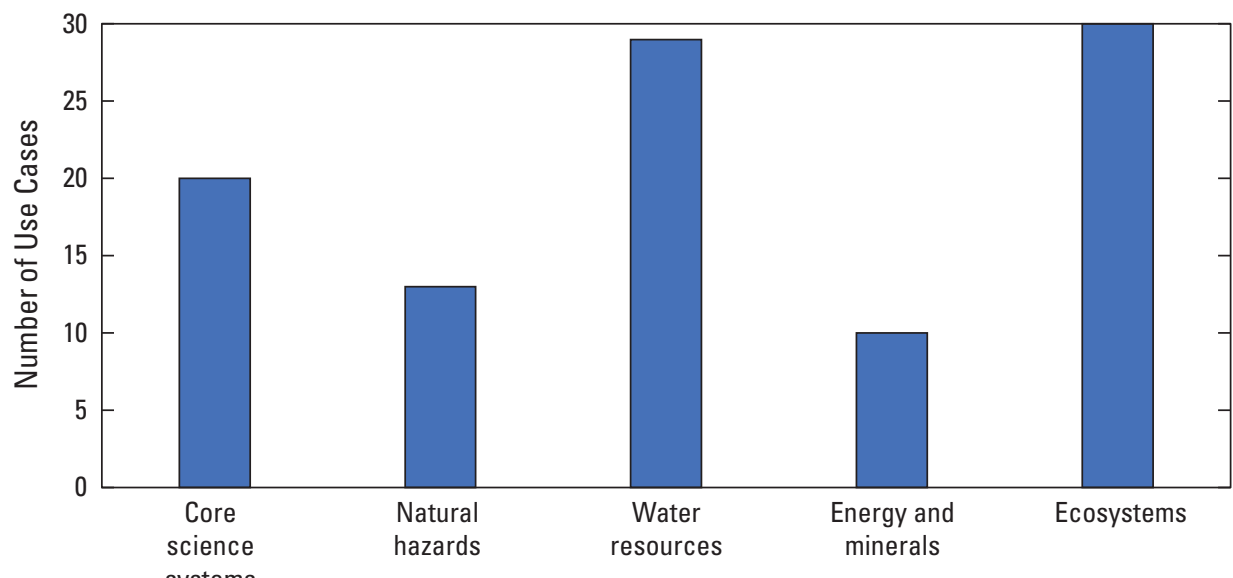

Mission area

Figure 3. U.S. Geological Survey Mission Areas represented in Use Cases. 


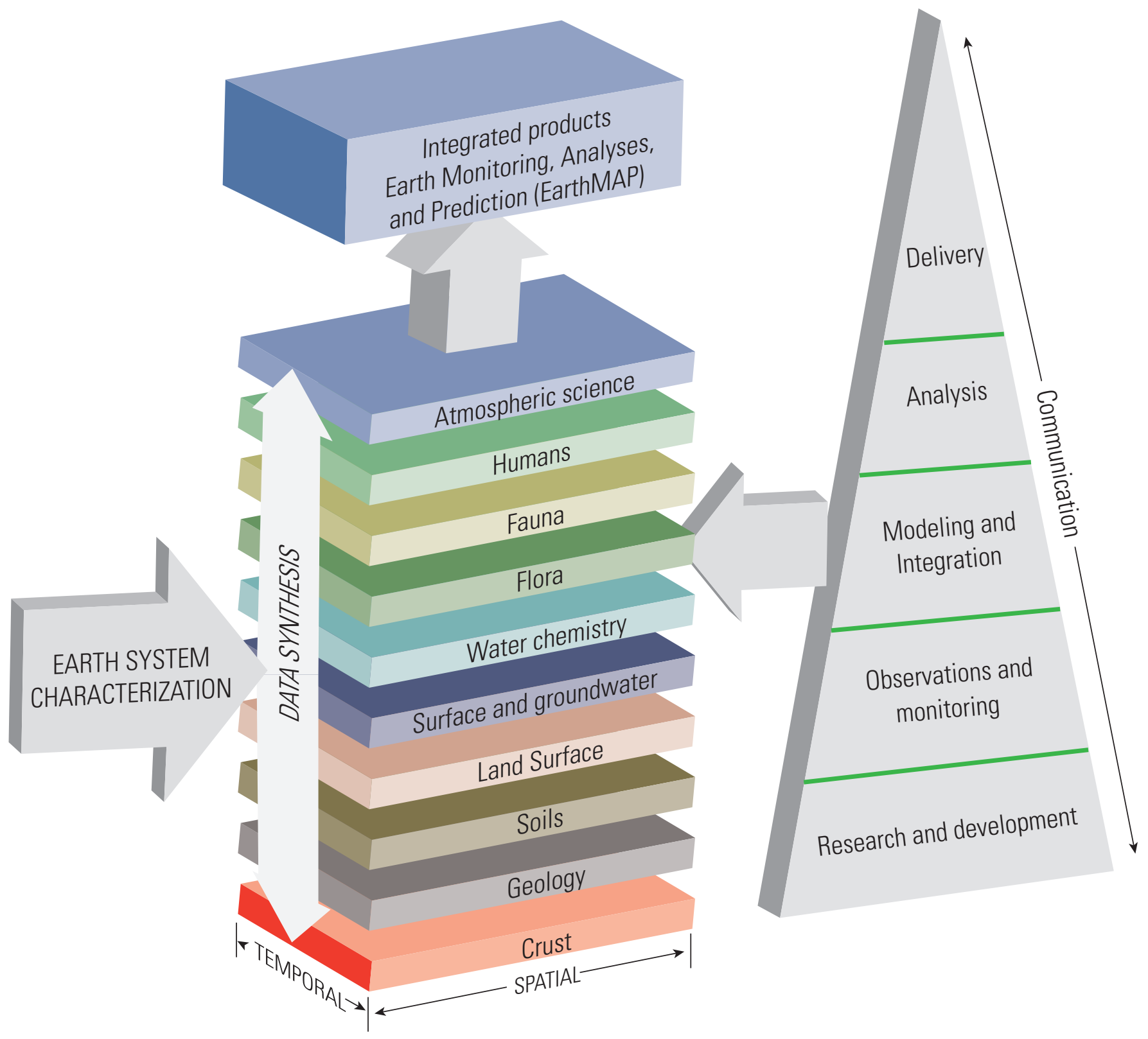

$\begin{array}{cccccccccc} & \text { Geology } & \text { Soils } & \begin{array}{c}\text { Land } \\ \text { Surface }\end{array} & \begin{array}{c}\text { Surface \& } \\ \text { Groundwater }\end{array} & \text { Flora } & \text { Fauna } & \text { Humans } & \begin{array}{c}\text { Atmospheric } \\ \text { Science }\end{array} \\ \text { \# of Use Cases } & 22 & 16 & 32 & 28 & 24 & 26 & 23 & 10\end{array}$

Figure 4. Conceptual illustration of Earth system characterization themes (modified from Jenni and others, 2017) in context of integrated science and the number of Use Cases in each theme. 


\section{Demand and Relevance}

\section{Specificity of the Identified Decisions and Decision Makers}

Use Cases varied in how clearly both the target decision(s) and the specific decision makers who would use the science output were specified. Of the Use Cases, 14 identified a general category of decisions as the decision-support goal, 12 described a general category, with at least one specific example, and 8 referenced specific decisions. In two instances, no target decision could be identified - these Use Cases described products that would advance situational awareness for scientists and decision makers but were not designed to specifically support decision makers. The complete set of 36 Decision Support Statements are included in appendix 6. Identification of specific decision makers was evident in less than half of the submissions, with a majority (24) of Use Cases providing only generalized descriptions of decision makers using information produced; 10 included a general category and at least 1 specific example of a decision maker.

\section{Common Types of Decisions Supported}

Several common types of decisions or topical decision areas were identified across the Use Cases, with most identifying more than one supported decision (fig. 5). The most common types of decisions identified were characterized as adaptation, mitigation, and response decisions (for example, how to increase the resilience of coastal communities to climate-related stressors and how to prevent or respond to harmful algal blooms). Other common types of decisions included water and land management decisions (including operational water management decisions such as reservoir operations and land use planning in the sagebrush biome);

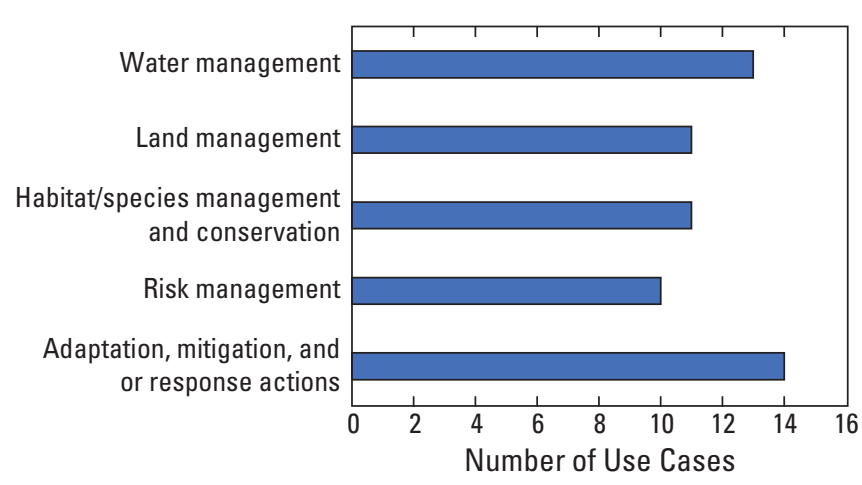

Figure 5. Characterization of the 36 Use Cases in terms of the topic type of decision support provided. Number of use cases targeting support for common decision types ( $n=36$, multiple decision types possible). decisions about how to manage and conserve habitats and species (for example, annual regulatory decisions on management of North American bird species); and risk management decisions (such as managing post-wildfire flood risks).

\section{Opportunities for Additional Integration and Collaboration from the Specific Use Cases Submitted}

Several areas stand out as opportunities for improved collaboration, from the perspective of the decision-supportive products and the general decision-maker categories identified.

- Four Use Cases centered around ecohydrology, three with a decision focus on water management and one with a decision focus on land management.

- Six Use Cases support decisions that would likely lead to water-quality improvements.

- Four Use Cases focus on land management and would likely provide enhanced singular or integrated data products for generically described decision makers (indicating stronger connections with decision-making partners are needed).

- Two Use Cases address habitat and species management and conservation as a specific decision focus, while many had overlapping decision spaces (that is, around water and land management and adaptation).

- Wildfire emerged as the top theme in terms of risk management decisions addressed by Use Cases across regions $(\mathrm{n}=5)$, indicating strength in USGS wildfire-related science.

- Adaptation, mitigation, and response decisions were supported by nearly all Use Cases, with strong overlap with risk management decisions, illustrating the need for information related to a wide variety of decisions in the context of change.

\section{Level of Stakeholder Engagement}

Three categories of stakeholder engagement were interpreted from the Use Case submissions, they are stakeholders that (1) work directly with the USGS to produce science products, (2) ask for or provide support for products, and (3) use USGS products. Figure 6 shows stakeholder engagement as a function of each category, with the majority having existing stakeholders and more than half having potential stakeholders asking for or providing support. For existing USGS products, known stakeholders were consistently identified. 


\section{Stakeholder Diversity}

To quantify stakeholder diversity, stakeholders were binned into five categories (fig. 7): (1) Department of the Interior (DOI) agencies; (2) other federal agencies; (3) state agencies; (4) tribal; and (5) other (including nongovernmental organizations, private, academia, and industry). Most of the Use Cases involved four or more stakeholder categories (fig. 8).

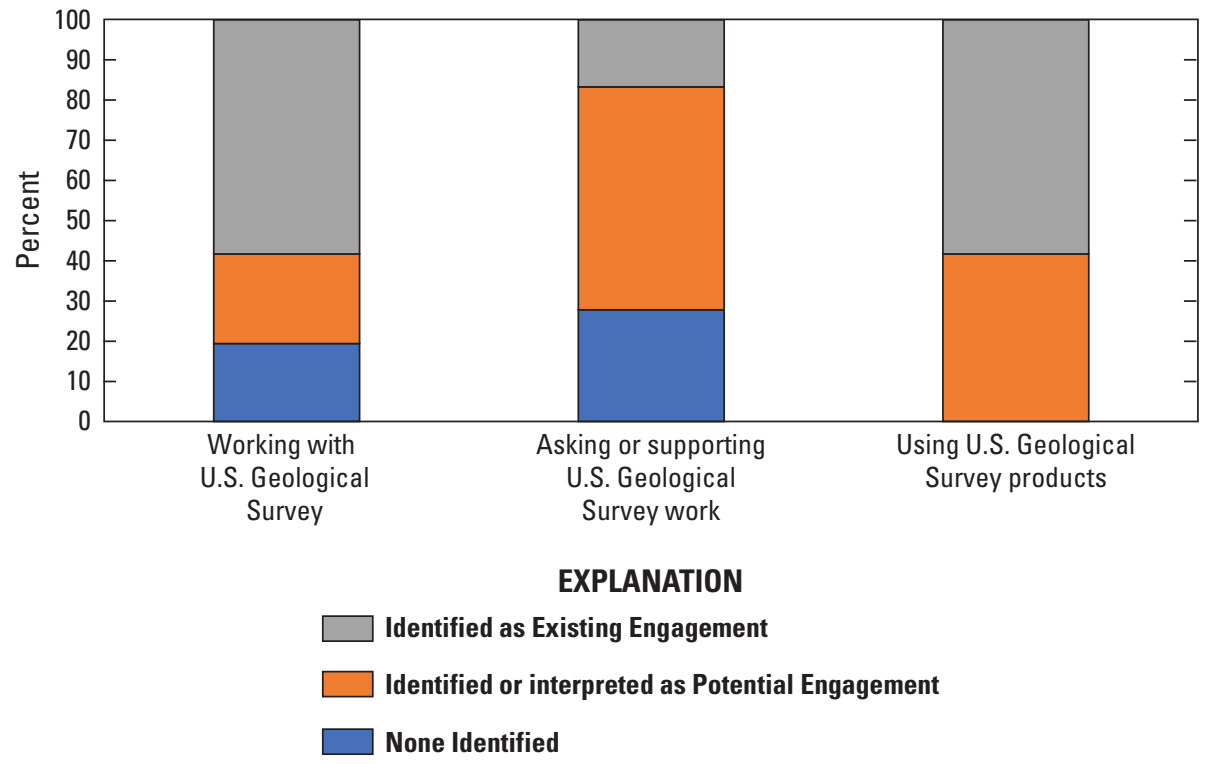

Figure 6. Stakeholder engagement by type of activity and level specified within Use Case summary.

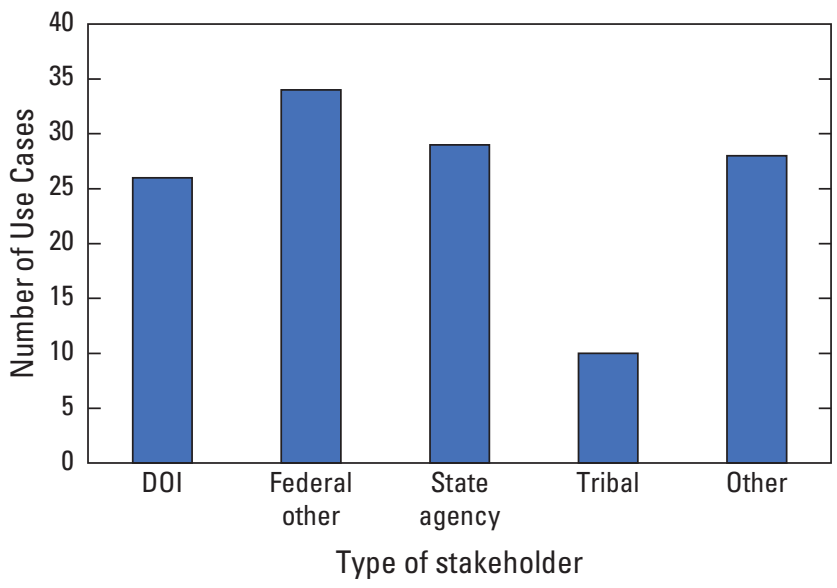

Figure 7. Stakeholder diversity expressed by number of Use Cases having different stakeholder categories.

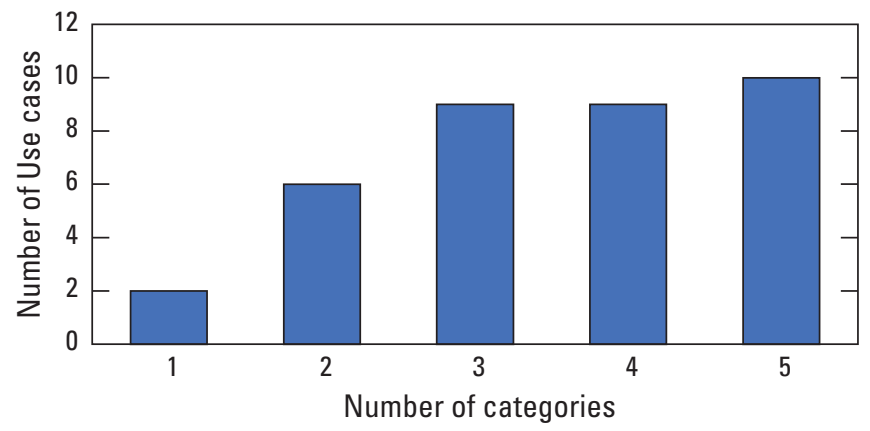

Figure 8. Distribution of number of stakeholder categories for submitted Use Cases. Number of stakeholder categories per Use Case. 


\section{Improving Decision Support}

\section{Nature of Decision Support Provided}

Just over a third of the Use Cases described products intended to provide actionable information to decision makers. These include Use Cases that aim to produce a narrowly defined product to support a specific set of decisions (for example, Harmful Algal Bloom Monitoring and Prediction [HABMAP]). Other Use Cases aim to integrate all the various kinds of data and predictions that would help inform more broadly defined decisions (for example, USGS Wildland Fire Science Program). Most of the Use Cases described products that would provide indirect support to decision makers. One third described information or products that could be used in combination with other information to support targeted decisions (for example, improved predictions of nutrient loading from the Connecticut River Basin to Long Island Sound, which provides partial information to support water-quality mitigation decisions). For other Use Cases, output of the science application would serve as input to subsequent models or analyses that, in turn, provide decision support (for example, improved estimates of snow water equivalents in high altitude environments, as one input to streamflow projections that support reservoir operations decisions).

\section{Science Capabilities and Advancement Opportunities}

\section{Use Case Author Capacity Assessment}

A limited follow-up survey of Use Case authors was conducted to evaluate the ability of the USGS to execute the proposed Use Cases with existing scientific and technological capabilities versus the need to develop such capacity within or outside the Use Case (to view full survey, see appendix 4). To facilitate a gap analysis between results of the follow-up survey and results from the EarthMAP Capacity Assessment (Keisman and others, 2021), the same capacity questions used for the CAT Survey were posed to the Use Case authors. Authors indicated an average intended use of 6 of the 19 data-collection methods, 8 of 10 data and information integration approaches, 7 of 10 modeling and prediction approaches, and all 4 capabilities to deliver actionable information included in the Capacity Assessment Survey. However, within the same categories, respondents indicated insufficient capacity for Use Case implementation in data collection methods (58 percent of respondents), data integration approaches (55 percent), modeling and prediction approaches (60 percent), and actionable information delivery (25 percent). Specifically, the greatest needs were centered around the use of unmanned autonomous vehicles, traditional ecological knowledge, and public mobile applications.
Data collection methods most commonly used and with the highest level of practitioner experience were remote sensing, light detection and ranging (LiDAR), hydroacoustic sensors, and use of camera traps; these methods were applicable in $28,21,11$, and 11 Use Cases, respectively (fig. 9). Methods with the least practitioner experience include biometric wearables, Deoxyribonucleic acid (DNA) chip technology, high throughput toxicity screening, and soundscape audio, which were each applicable in four or five Use Cases (fig. 9).

The greatest needs for predictive science included improved uncertainty estimates for decision making $(n=20)$, coupling/integration of multiple models $(n=17)$, and operational ecological forecasting ( $\mathrm{n}=18$; fig. 10). Additional barriers to implementation included lacking capacity in decision science $(\mathrm{n}=11)$, data delivery applications and visualization $(\mathrm{n}=11)$, co-development of projects with stakeholders $(\mathrm{n}=6)$, and experience in engaging with stakeholders $(\mathrm{n}=4)$.

\section{Science and Technology Capacity Gap Analysis}

Results of a capacity gap analysis comparing the Use Case Author Capacity Assessment ( $\mathrm{n}=33$ ) and the Bureau-wide Capacity Assessment (1,035 respondents; Keisman and others, 2021) revealed more similarities than differences. This pattern is evident in the generalization of capacity assessments group by EarthMAP components (figs. 11A, B), where inverse responses between surveys indicate similarities in assessment of capacity within the Bureau and needs specifically for Use Cases. Results indicated that more capacity is needed in data and information integration and in modeling and prediction approaches relative to that needed for actionable information delivery (figs. 11A, $B$ ).

There were limited examples of conflicting needs with respect to data and information capabilities from comparison of the two capacity assessments (table 7). Combined results indicated where capabilities were assessed to be sufficient for current science demands and insufficiencies where they disagree. Remote sensing, LiDAR, hydroacoustic sensors, and camera traps were data collection methods most needed for Use Cases and with the highest level of overall USGS practitioner experience, which align with Use Case science application needs.

Results from the Use Case Author Survey indicated five research skills ranked in the highest quartile of the "Advanced" practitioner category: Inter-agency collaboration, data visualization, science communication, reducing uncertainty in management decisions, and stakeholder engagement. Cross-survey comparison reveals an opportunity to increase capacity for modeling approaches, such as statistical enumerators, model-data fusion, and Artificial Intelligence/Machine Learning (AI/ML) for future USGS science. 
Results

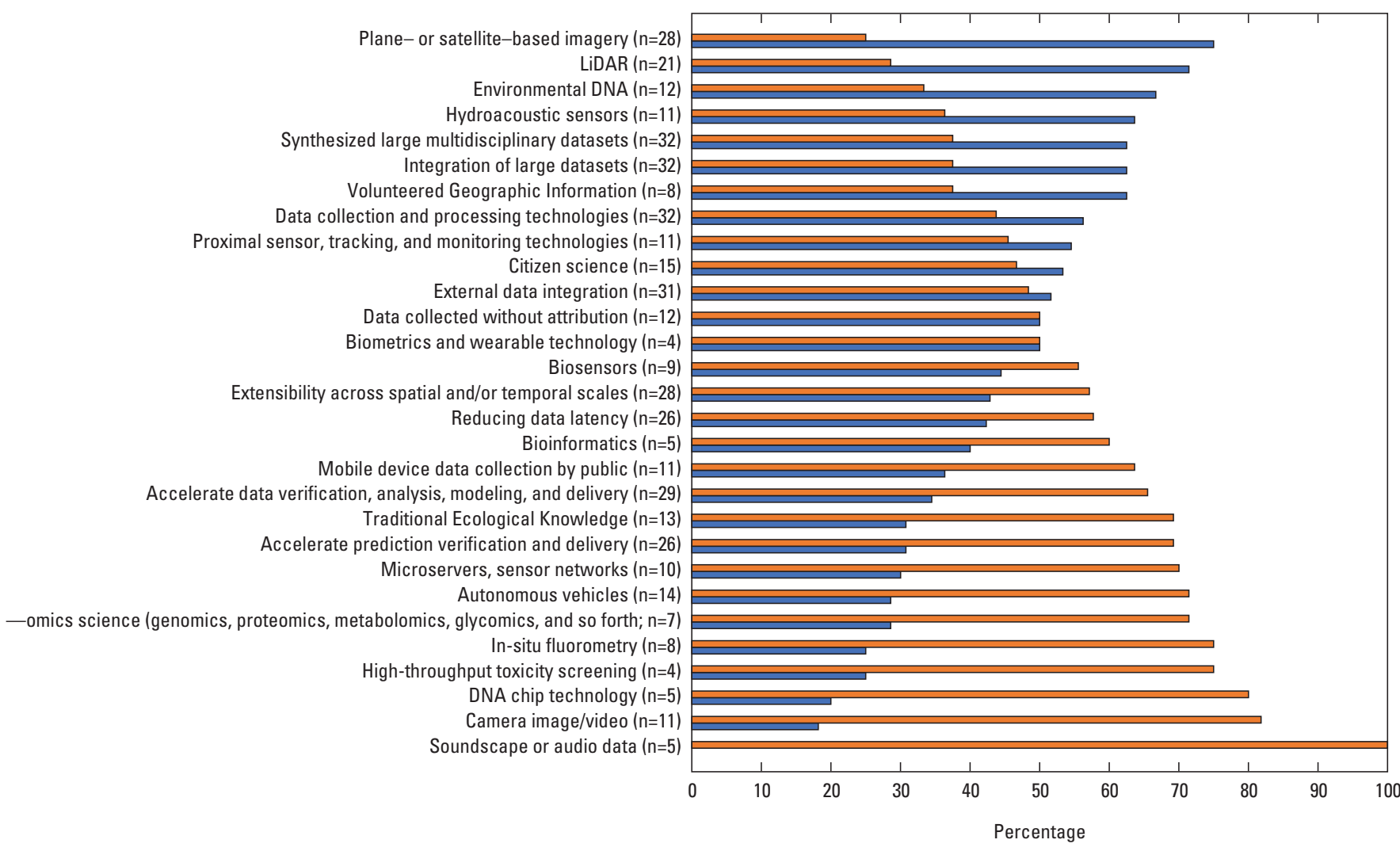

EXPLANATION

$\square$ Not sufficient $\square$ Sufficient

Figure 9. Use Case author assessment of whether current known capacity was sufficient for data and information integration capabilities. In parentheses, $\mathrm{n}$ is the number of Use Cases indicating applicability out of 33 responses. Abbreviations: LiDAR, Light detection and ranging; DNA, deoxyribonucleic acid. 


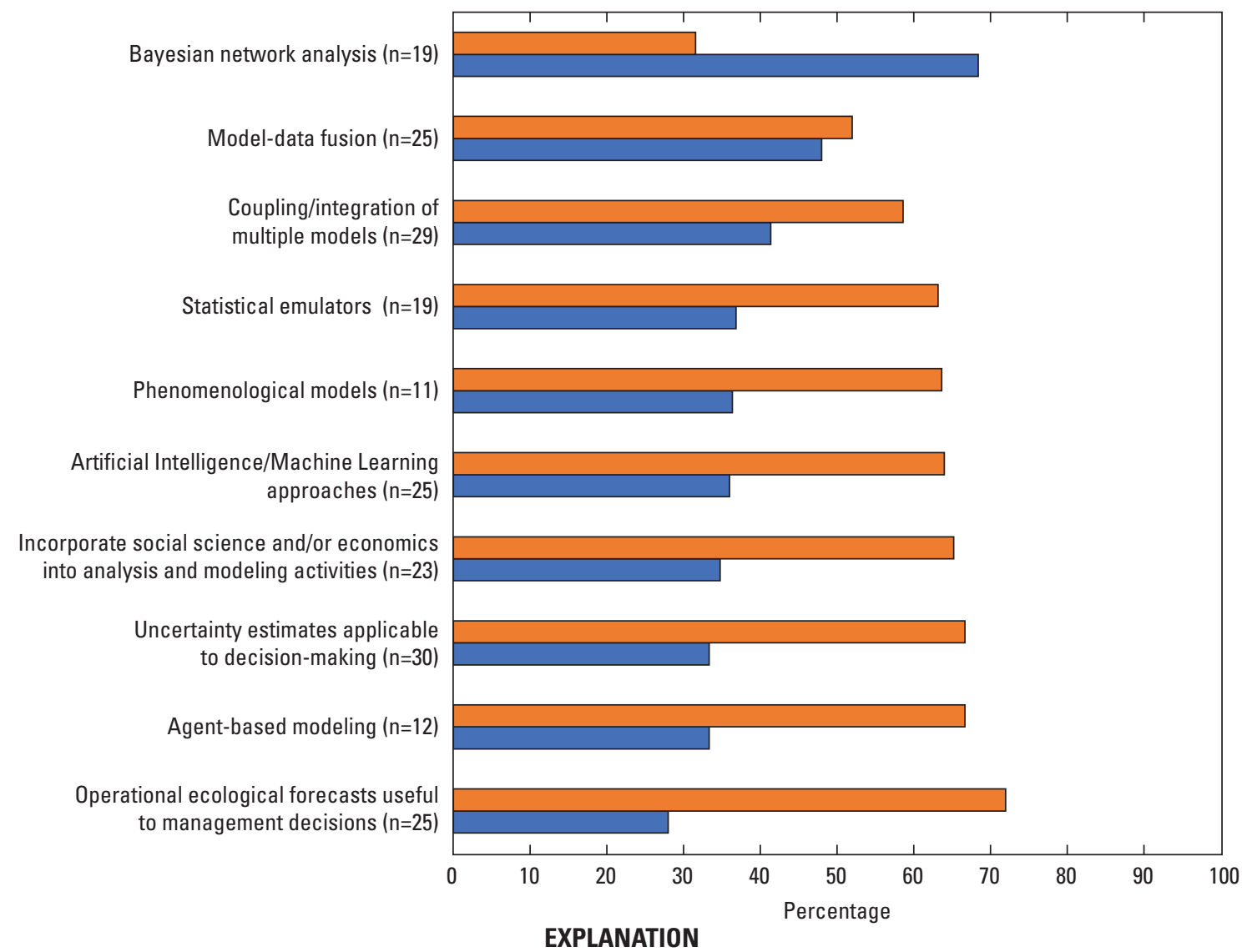

Not sufficient $\square$ Sufficient

Figure 10. Use Case author assessment of whether current known capacity was sufficient for applicable modeling and prediction capabilities. In parentheses, $\mathrm{n}$ is the number of Use Cases indicating applicability out of 33 responses. 

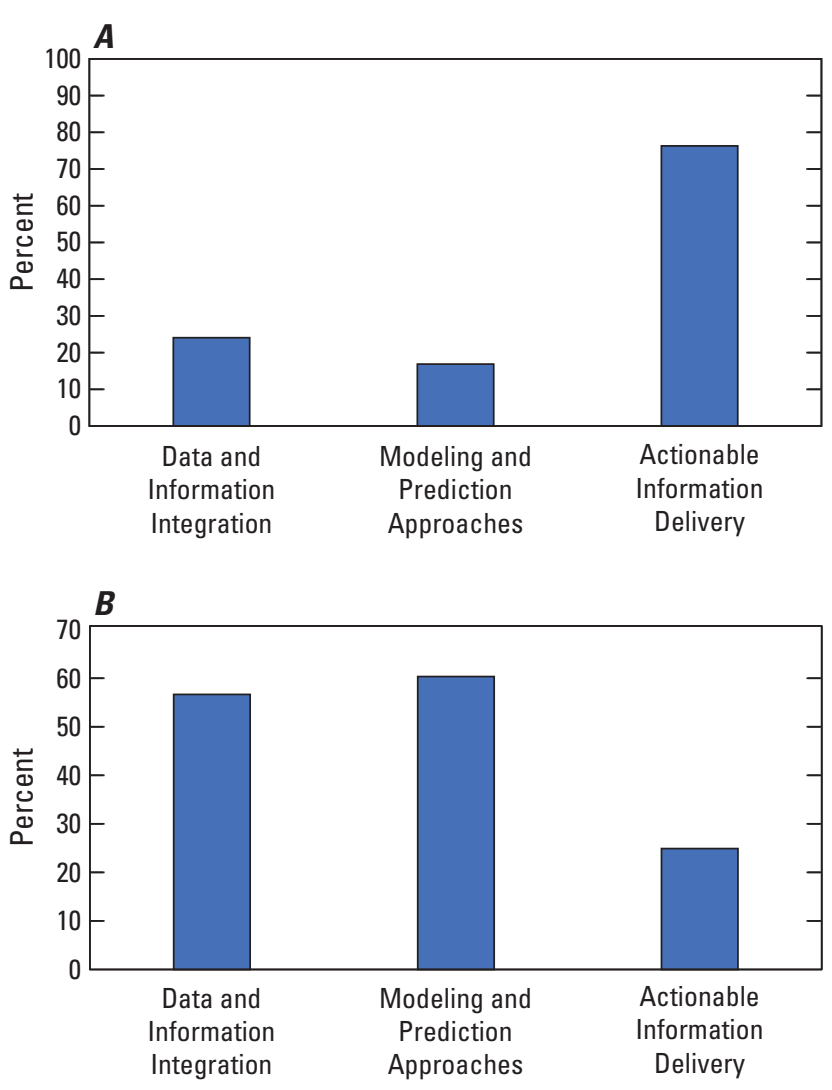

Figure 11. A, Percent of Capacity Assessment Team (CAT) Survey responses indicating some level of experience averaged for capabilities within groupings by the three EarthMAP components. $B$, Percentage of Use Case authors indicating known capacity is insufficient to implement their Use Case. It is important to note that the CAT Survey had 1,035 respondents, whereas the Use Case author survey had 33 respondents. CAT Survey results presented in Keisman and others (2021).

CAT Survey results for modeling approaches, such as statistical enumerators (37.4 percent, $\mathrm{n}=163$ ), model-data fusion (35.2 percent, $\mathrm{n}=154$ ), and AI/ML (34.4 percent, $\mathrm{n}=150$ ) (Keisman and others, 2021) compared to Use Case author responses (fig. 10), indicate the applicability and potential to increase capacity in these areas for future USGS science. Bureau-wide survey results indicated that 41.5 percent $(n=430)$ of respondents deliver data applications and visualizations on the web compared to all 33 responding Use Case authors indicating skills and experience in data applications and visualizations in the described science application and 33 percent indicating that known current capacity is insufficient.

One conclusion from this comparison is that Use Case capacity gaps could potentially be met by existing bureau-wide capacity. This finding confirms the utility of both surveys because both are consistent in identifying areas of insufficient or sufficient capacity. However, survey comparison discrepancies also emerged, which included capacity and expertise in camera traps, unmanned autonomous vehicles/ drones, environmental DNA (eDNA), and uncertainty estimates applicable to decision-making expertise. These discrepancies could be attributed to a lack of information across mission areas regarding staff working in these areas. Although capacity gaps do exist, with time, resources, creative problem solving, and focused coordination across regions and mission areas, it appears the USGS has existing capacities that can be leveraged to meet most of the capacity needs described in the regional Use Cases.

The Use Case Author Capacity Survey included additional open-ended text input fields to gather feedback from authors outside of the confines of direct linkages to the CAT Survey. A word cloud summary of identified predictive science capacity needs highlights the technology driven capacities required to conduct USGS science now and into the future (fig. 12).

\section{Advancement Opportunities}

Use Case development guidance requested that Use Cases improve science-informed decision making related to substantial societal issues and demonstrate advancement in USGS monitoring, analysis, and predictive capacity. All Uses Cases identified advancement opportunities; though, some lacked detail in the pathway toward that opportunity, the majority provided a clearly defined pathway. Information advancements for improved decision making were identified for (1) information accuracy and resolution $(\mathrm{n}=28),(2)$ scope and value of information to the decision $(\mathrm{n}=36)$; (3) timeliness of information delivery ( $\mathrm{n}=29)$, and (4) accessibility and usability of information $(\mathrm{n}=33)$.

\section{Operational Implementation and Technical Considerations}

Use Cases fell into two different stages of maturity: extension of current projects $(n=13)$ and new projects $(n=23)$. Overall, the UCDT estimates that 16 of the Use Cases could be implemented within 3 years if funding were available. Major impediments to implementation were characterized as cost ( 89 percent), capability gaps (61 percent), personnel (44 percent), current prioritization (36 percent) and other impediments, including data storage (17 percent).

\section{Longevity and Sustainability}

Nearly all Use Cases met or exceeded the characterization criteria for long-term projects that would be sustainable over time. The majority of the Use Cases could be scalable nationally, nearly half were determined to have sub-national applicability, a third were regional in scope, whereas only a few were limited to localized applications. In summary, seven Use Cases were determined to have broad applicability ranging from the local to national scale. 


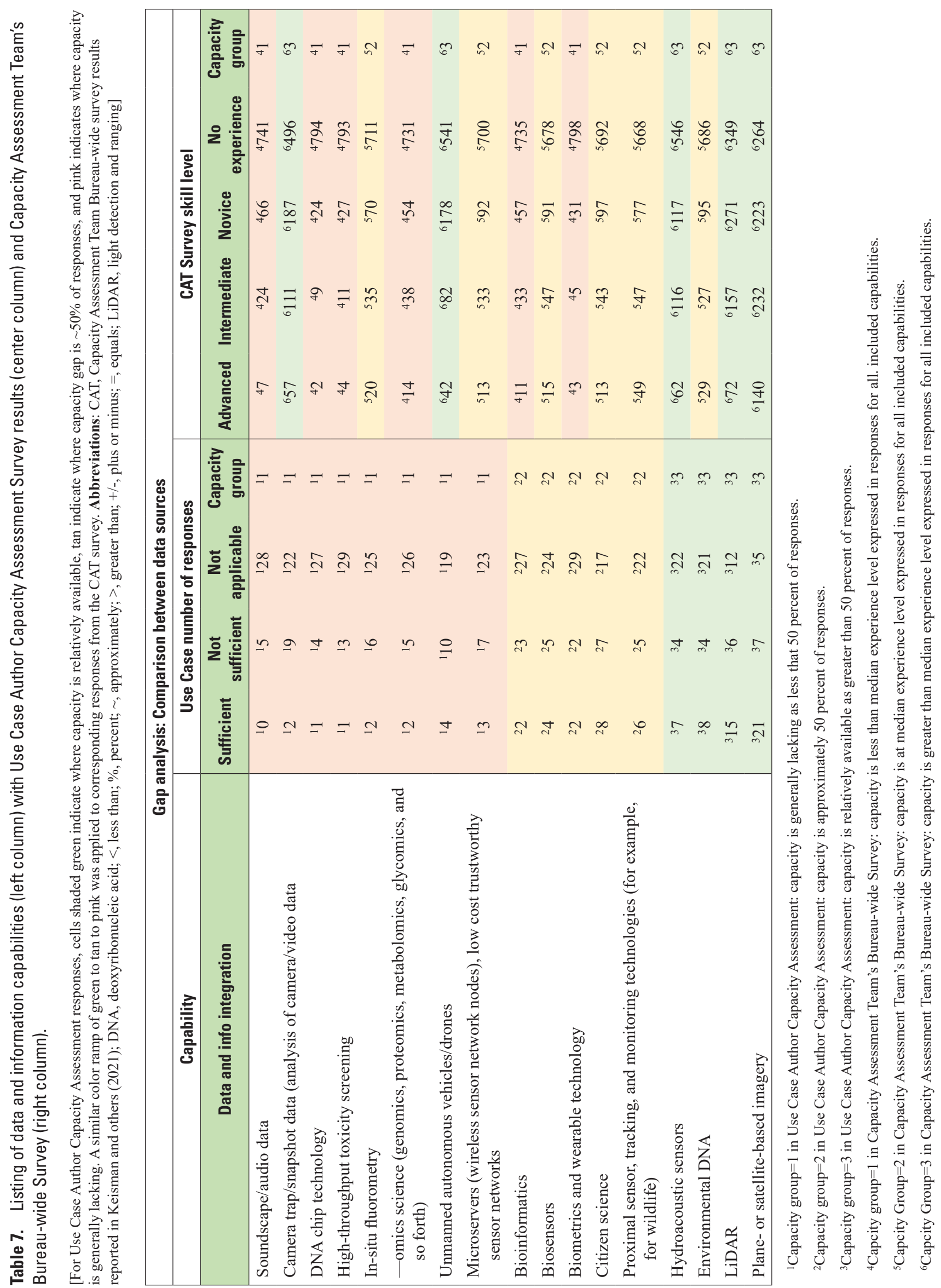




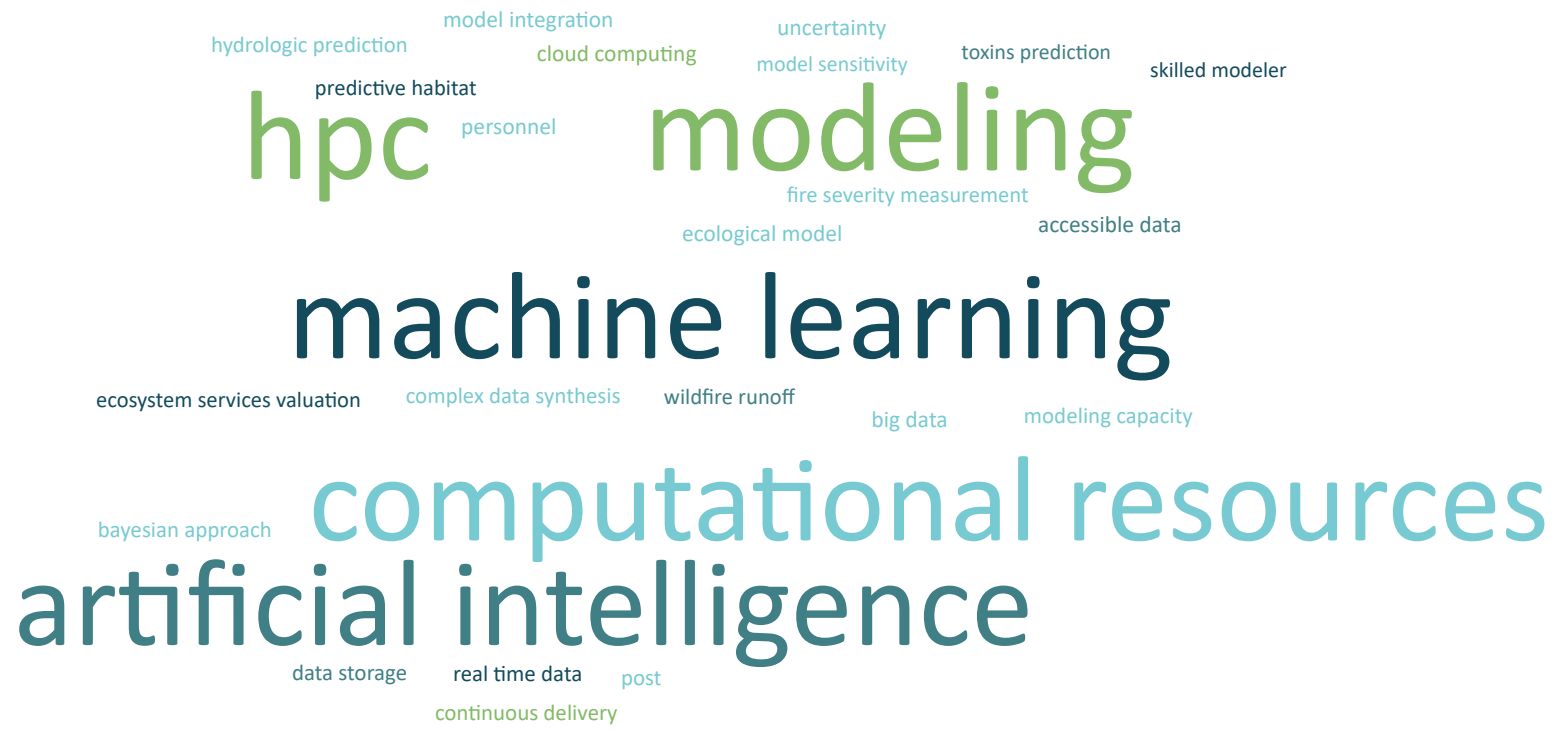

Figure 12. Summary of terms, shown as a word cloud, related to capacities for Use Case predictive science identified by Use Case authors.

\section{Geographic and Scientific Convergences}

The UCDT analyzed potential points of scientific convergence and commonalities among the Use Cases that could provide connections among participating scientists. The Southwest and Rocky Mountain Regions asked respondents to give special consideration to Use Cases with direct applicability to the Colorado River Basin. Seven Use Cases specifically named the Colorado River Basin as a focal geographic area. Coastal hazards and coastal resilience were common scientific themes in Use Cases from the Alaska, Northeast, and Southeast Regions. Scientific applications associated with wildfire and post-wildfire effects were common in Use Cases from western Regions (Alaska, Rocky Mountain, and Southwest Regions). Significant topical convergence centered around connections between water availability and quality, as well as the quality of habitats and ecosystems for wildlife ( $\mathrm{n}=13$ ), which are evident in the keyword analysis (fig. 13).

\section{Common Themes}

The primary objective of Use Case science applications centered around resource protection and hazard planning and mitigation (fig. 14). Applications focusing on resource protection addressed topics ranging across all mission areas, including water, species, ecosystem services, and minerals. Anthropogenic concerns (life and property) and ecosystems and water resources were common hazard application objectives, as were wildfire-related activities.
Regulatory/legal compliance requirements and climate change also emerged as significant common themes as secondary objectives and topics. A possible explanation for this convergence is that many water and ecosystem resources fall under some type of governmental protection or disbursement, and stakeholder interest peaks when consequential climate change impacts occur or are predicted to occur.

\section{Administration's Priorities}

Many of the Use Cases align with and could support the current (2021) Administration's DOI priorities (U.S. Department of the Interior, 2021; The White House, 2021; Executive Order 13985, Executive Order 13990, Executive Order 14008, Executive Order 2021; National Climate Task Force, 2021). At least 29 Use Cases could support activities related to conservation of public land and waters (for example, systematic data collection, synthesis, and predictions designed specifically to support the National Parks Service Resource Stewardship Strategies); 16 relate to climate science (for example, an effort to synthesize existing products to describe the intensity of anthropogenic change and predict its implications for natural communities and ecological services in the Pacific Northwest); 13 will produce information that could support economic recovery, jobs, or infrastructure (for example, integrating geospatial information on, above, and below ground minerals resources); and at least 8 could provide science information and support for underserved communities, aligning with DOI social justice priorities (for example, co-producing coastal hazard assessment and mitigation strategies with Bering Sea communities of Alaska). 
edna pathogens tracking

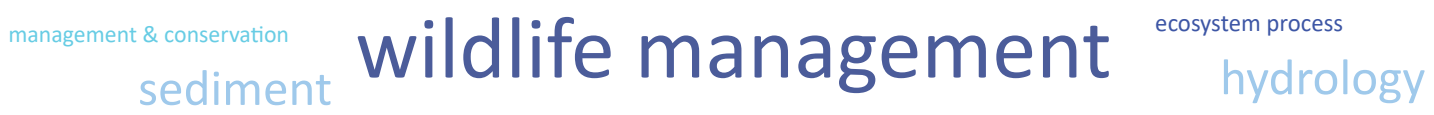

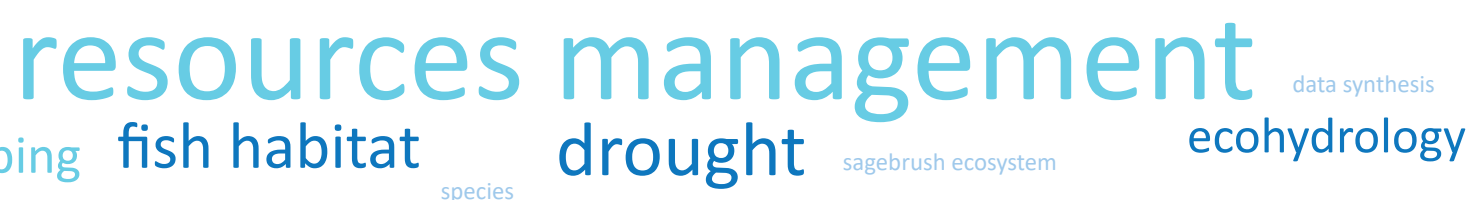

mapping fish habitat drought sagerushecosystem ecohydrology

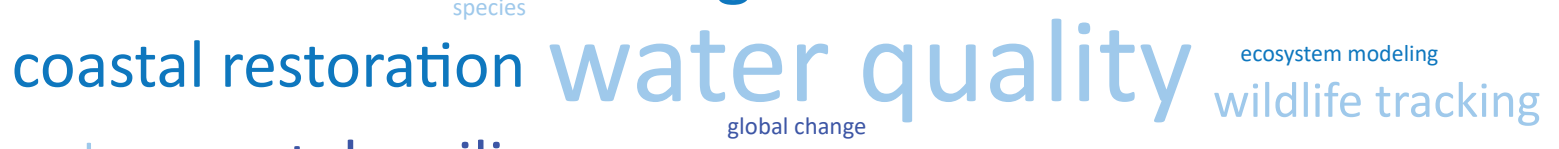

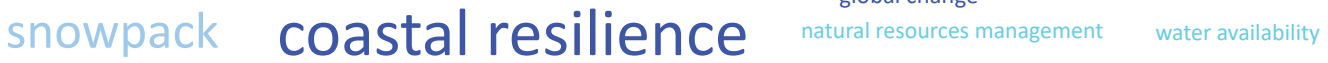

volcanic hazard nutrients loading Water availability

energy development hazard

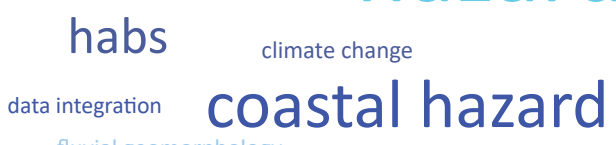

minearlesources

water management

habitat management

Figure 13. First-order keywords and phrases, shown as a word cloud.

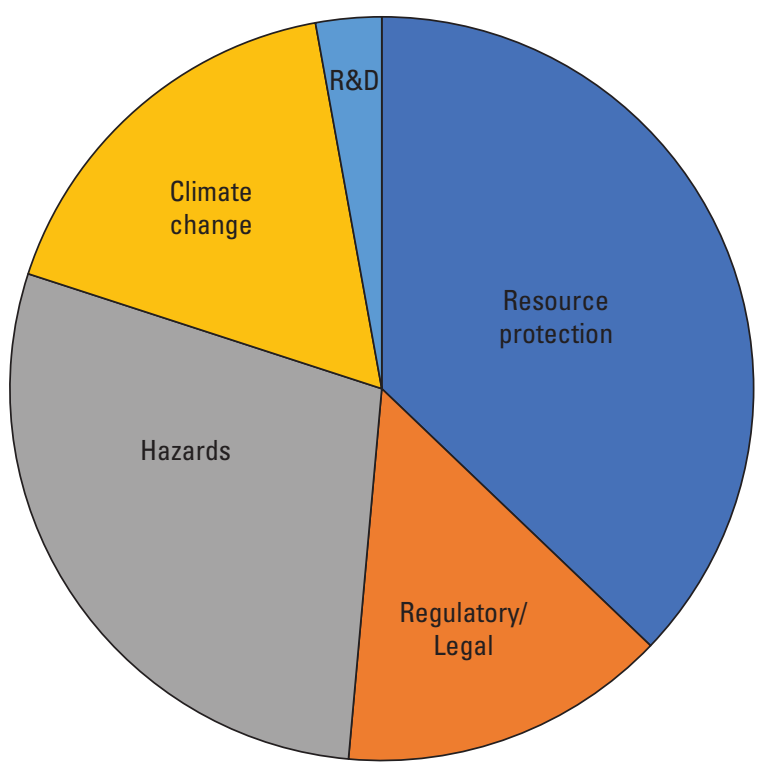

Figure 14. Common primary objective motivators based on data or information to be developed for submitted Use Cases.

\section{Grand Challenges}

Most of the Use Cases were aligned with one or more USGS Grand Challenge (Jenni and others, 2017), broadly defined as natural resource security, societal risk from existing and emerging threats, smart infrastructure development, and anticipatory science for changing landscapes. These Grand Challenges are useful in inspiring new science and technology for advancing a vision for integrated predictive science, yet were not interpreted as a strict organization of activities. For example, Use Case alignment with multiple Grand Challenges can identify existing or needed enterprise capacities for a broad range of activities. The UCDT noted the lowest number of Use Cases aligned with the smart infrastructure development Grand Challenge, while acknowledging that the Use Cases authors were not directed to these Grand Challenges or asked to consider them in Use Case development. Nonetheless, the resulting alignment is evidence of the broad applicability and representativeness of the Grand Challenges for USGS science. 


\section{Discussion}

\section{Recommendations for Improving the Use Case Solicitation Process}

The Use Case development process documented in this report was the first effort of its kind in the USGS. Challenges encountered during the process included (1) continuous education and outreach to facilitate understanding of an integrated predictive science vision, (2) EarthMAP's tentative funding and long-term program status, and (3) the novel coronavirus disease-2019 (SARS-CoV-2), which disrupted plans for face-to-face meetings with internal and external stakeholders. Despite these challenges, the following lessons were learned:

1. Use Case solicitation should begin by clearly defining the purpose of the Use Case solicitation.

2. Clear guidelines are needed to address how the Use Cases will be evaluated and then the submission form can be modified to directly align with the information needs.

3. Standardized, pre-defined menus (such as pull-down menus for information such as mission area, topics, themes, capacities, number of stakeholders, list of stakeholders, timeframe to implement) should be used in future Use Case templates to facilitate characterization directly from authors, rather than to rely on subsequent subjective evaluation, such as from the UCDT. Options for narratives could be added as supplemental information.

4. More value with respect to defining more complete Use Cases, and resulting strategic planning for integrated science opportunities could be gained from asking specific questions with respect to the following information:

- Level of stakeholder engagement

- Barriers to success (such as funding, time, full time employees, and so forth)

- Improved definition and self-identification of project maturity

- Timeline to produce actionable information must be better defined, including estimated time to launch

- Types of information to be collected and delivered

- Types of decisions informed

- Prioritization of information value

- Identification of the types and numbers of science partners (distinguish partner from stakeholder)
- Data types required for successful implementation

5. Amending and providing specific guidance for Use Case development and submission, such as the following:

- Guidance for decision support statements written directly by Use Case authors (see examples in appendix 6)

- Clearly describe the concept of operations for the science application

- Delineate how long the decision space informed by the science will remain relevant

- Length of Use Case submission (4-page limit) should be extended

- A complete Use Case submission example document would benefit future submissions and provide developers with insight into the most salient types of information

- Combine the submission with a capacity survey

\section{Recommendations from the Gap Analysis}

Results of the gap analysis presented in this report were limited in scope, yet useful. The general conclusions are:

- The USGS uses a variety of data collection methods; though some have higher demand and are more broadly applicable (for example, remote sensing, LiDAR, and eDNA), others provide critical data for specific science applications (for example, biometric wearables, DNA chips, and high throughput toxicity screening).

- There is a clearly expressed need for increasing capacity for data processing workflows to improve the speed of USGS science.

- There is a demand for improving USGS capabilities and capacities in using, integrating, and synthesizing large datasets for purposes of modeling and prediction.

- Current modeling and prediction approaches are insufficient for successful Use Case implementation, which reflects the increasing demand for these skills to meet the requirements of integrated, predictive science.

- The capability to deliver actionable information was relevant to most Uses Cases, with fewer gaps in capacity observed in this area, but awareness of those needs might increase with further engagement in co-development activities with partners for integrated, predictive science. 
Four critical Use Case capacities in data and information integration were most apparent as gaps where there is a potential large return on investment due to their applicability to Use Cases and advancement in applied earth science, irrespective of topical domain:

1. Develop processes to reduce data latency

2. Develop approaches that increase speed of data verification, analysis, modeling, and delivery

3. Enhance extensibility of research and monitoring insights across spatial and temporal scales

4. Increase the speed of prediction verification and delivery

\section{Potential Collaborative Opportunities}

Identification of potential collaborative opportunities for 21st-century science that span multiple USGS centers, regions, scientific disciplines, and mission areas resulted from Use Case development. Although the Use Case submissions were relatively brief, their content and subsequent information from the Use Case Author Capacity Assessment Survey results revealed several areas that warrant further examination for future USGS activities. Looking nationally across geographies and disciplines illuminates opportunities for collaboration and leveraging of financial and intellectual resources to increase the reach and impact of USGS science.

Not surprisingly, most Use Cases identified initial target geographies of interest that are native to the originating region. However, most Use Cases indicated that their approach could be applied to multiple regions and half were judged to be extensible to a national scale. The most significant topical convergences were connections between water availability and quality and quality of habitats and ecosystems support for wildlife. In addition, there is a clearly stated need for regional water models linking groundwater and surface-water flows, sediment dynamics, and physical geography to the distribution and quality of habitats and ecosystem services supporting wildlife. These models could be applied or shared among many national restoration programs with strong USGS involvement, such as the Sacramento-San Joaquin Delta, the Florida Everglades, the Chesapeake Bay and Watershed, the Delaware River system, and Puget Sound/Salish Sea. At least 13 of 36 submissions converge on this specific interest.

\section{Recommendations from Characterizing Use Cases Through the Lens of Decision Support}

The Use Case submission template included a section for describing how the proposed science application would improve decision making: "Use Cases will identify what aspect of the decision-making process is targeted for improvement (for example, information accuracy and resolution, information scope and value, timeliness of delivery, accessibility and useability) and how that fits into decision and action frameworks." However, this perspective was clearly not natural for many authors because almost all summaries focused on the proposed science questions and activities rather than on the specific details surrounding decisions, decision support, and actionable information to be delivered.

Future regional efforts would likely benefit from (1) a refined format and template for defining the decisions and decisions makers to be supported and resulting decision-support products (similar to the summary tables shown in appendix 5) and (2) co-development of these ideas with decision-making partners or other stakeholders to refine the decision focus.

\section{Opportunities}

The need for interdisciplinary science that provides an understanding of complex earth science systems has been recognized for decades. Moreover, interdisciplinary science continues to be frequently sought by today's resource managers and decision makers. The USGS, with its multidisciplinary capacity, is ideally suited to meet such needs for interdisciplinary science. Increasing USGS delivery of societally relevant integrated science, at the scales and in the timeframes necessary to support decision making, is perhaps the greatest opportunity to increase the impact of the USGS.

The Use Cases provide powerful examples of how a strategic focus on integrative data and science, on predictive capacities, and on providing science that can be acted upon, will deliver new value to our decision-making partnersespecially when those partners are actively involved in the co-development and co-production of science. Each Use Case presents a realistic opportunity to expand USGS science and support decision making. The UCDT process of organizational Use Case development identified several near-term opportunities for the mission areas and the regions to collaborate with each other and with decision-making partners to continue to develop integrated predictive science concepts as part of a vision for future USGS science: Leveraging existing capabilities, building new enterprise capabilities and capacities, and providing actionable information needed to support the complex resource management decisions society faces. 
Opportunities include:

1. Combine forces to address shared interests in common themes across regions, including direct involvement of decision-making partners and stakeholders.

2. Increase coordination for USGS wildfire science and impacts, coastal hazards and resilience, risk management decision making, and decision support with a focus on improving water quality.

3. Increase collaboration with land and water management at the interface of hydrology and ecology (ecohydrology), a geographic focus in the Colorado River Basin, and potentially identifying common science needs related to adaptation and mitigation decision analyses.

4. Improve clarity on connections between the proposed science activities and the decisions the work is intended to support - the inclusion of decision-making partners in future scoping of collaborative activities will increase the utility of these projects.

5. Leverage the Use Cases to identify and target investments in activities that relate to Bureau and Administration priorities. Programs, regions, and centers should consider these Use Cases as they develop new projects in response to Bureau and mission area annual guidance.

6. Invest in USGS capacity-building activities by comparing the capacities needed for implementation of the Use Cases with the capacity strengths and weaknesses identified by the EarthMAP Capacity Assessment (Keisman and others, 2021).

7. Improve modeling and prediction capacity by leveraging existing expertise across the Bureau for any newly proposed work to increase agency efficiency.

8. Raise awareness of common integrated and interdisciplinary science interests within and across the regions. Use Cases revealed many common and overlapping themes; the Capacity Assessment Survey
(Keisman and others, 2021) identified common and rare skillsets within the USGS. Results of these efforts provide the opportunity to share feedback with the regions and USGS scientists and to assist in identifying new connections.

The following recommendations emerged for consideration as part of institutional change that could facilitate bureau-wide integrated science moving forward:

1. Coupling appropriated and reimbursable funding to priority integrated science activities that meet stakeholder information needs,

2. Aligning independent research efforts within programmatic priorities where science could be applied, and

3. Ensuring the full capacity of USGS science is applied to priorities through inter-mission area collaboration and co-development with decision-making partners.

\section{Conclusions}

Increasing the capacity to deliver societally relevant integrated science, at the scales and in the timeframes necessary to support decision making, is perhaps the greatest opportunity to increase the impact of USGS science in coming decades. The Use Cases developed and presented in this report represent substantial thematic convergence related to conservation of public lands and waters, which is a powerful linkage lending strength to future collaborative efforts. Many of the capacity demand gaps identified by the Use Cases could potentially be met by existing bureau-wide capacity. Nearly half of the Use Cases could potentially be implemented within 3 years if funding, capabilities, and personnel impediments were removed as well as priorities shifted. Overall, the proposed science applications have the potential to improve decision making by advancing information accuracy and resolution, expanding the scope and value of information, increasing the timeliness of information delivery, and improving information accessibility and usability. 


\section{References Cited}

Executive Order 13985, 2021, Advancing racial equity and support for underserved communities through the federal government: Document \# 2021-01753, January 25, 2021, p. 7009-7013, https://www.federalregister.gov/documents/ 2021/01/25/2021-01753/advancing-racial-equity-andsupport-for-underserved-communities-through-the-federalgovernment.

Executive Order 13990, 2021, Protecting public health and the environment and restoring science to tackle the climate crisis: Document \# 2021-01765, January 25, 2021, p. 7037-7043, https://www.federalregister.gov/documents/ 2021/01/25/2021-01765/protecting-public-health-andthe-environment-and-restoring-science-to-tackle-theclimate-crisis.

Executive Order 14008, 2021, Tackling the climate crisis at home and abroad: Document \# 2021-02177, January 27, 2021, p. 7619-7633, https://www.federalregister.gov/ documents/2021/02/01/2021-02177/tackling-the-climatecrisis-at-home-and-abroad.

Jenni, K.E., Goldhaber, M.B., Betancourt, J.L., Baron, J.S., Bristol, R.S., Cantrill, M., Exter, P.E., Focazio, M.J., Haines, J.W., Hay, L.E., Hsu, L., Labson, V.F., Lafferty, K.D., Ludwig, K.A., Milly, P.C., Morelli, T.L., Morman, S.A., Nassar, N.T., Newman, T.R., Ostroff, A.C., Read, J.S., Reed, S.C., Shapiro, C.D., Smith, R.A., Sanford, W.E., Sohl, T.L., Stets, E.G., Terando, A.J., Tillitt, D.E., Tischler, M.A., Toccalino, P.L., Wald, D.J., Waldrop, M.P., Wein, A., Weltzin, J.F., and Zimmerman, C.E., 2017, Grand challenges for integrated U.S. Geological Survey science-A workshop report: U.S. Geological Survey Open-File Report 2017-1076, 94 p., https://doi.org/10.3133/ofr20171076.
Keisman, J.L., Bristol, S., Brown, D.S., Flickinger, A.K., Gunther, G., Murdoch, P.S., Musgrove, M., Nelson, J.C., Steyer, G.D., Thomas, K.A., and Waite, I.R., 2021, Capacity assessment for EarthMAP and future integrated monitoring and predictive science at the U.S. Geological Survey: U.S. Geological Survey Open-File Report 2021-1102, 110 p., https://doi.org/10.3133/ofr20211102.

National Climate Task Force, 2021, Conserving and restoring America the beautiful: U.S. Department of the Interior, 23 p., https://www.doi.gov/sites/doi.gov/files/reportconserving-and-restoring-america-the-beautiful-2021.pdf.

Ramsden, A., and Bate, A., 2008, Using word clouds in teaching and learning: United Kingdom, University of Bath, https://purehost.bath.ac.uk/ws/portalfiles/portal/ $378516 /$ using $\% 2520$ word $\% 2520$ clouds $\% 2520$ in $\% 2520$ tea ching \%2520and \%2520learning.pdf.

The White House, 2021, The Biden-Harris administration immediate priorities: The White House, accessed July 29, 2021, at https://www.whitehouse.gov/priorities/.

U.S. Department of the Interior, 2021, Our priorities: U.S. Department of Interior, accessed July 29, 2021, https://www.doi.gov/ourpriorities.

U.S. Geological Survey, 2021, U.S. Geological Survey 21st-century science strategy 2020-2030: U.S. Geological Survey Circular 1476, 20 p., https://doi.org/10.3133/cir1476. 


\section{Appendix 1. Earth Monitoring, Analysis, and Prediction (EarthMAP) Use Case Summaries-Guidance and Template Document}

The U.S. Geological Survey Earth Monitoring, Analysis, and Prediction (EarthMAP) vision emphasizes a forward looking, innovative USGS science portfolio able to deliver actionable information for our stakeholders through 21st-century approaches to monitoring, analysis, and prediction. EarthMAP is the mechanism the USGS will develop to facilitate and apply integrated, multidisciplinary science and technological advancements to meet decision-maker and stakeholder needs. Use Cases - as building blocks for EarthMAP — will identify high-priority science applications that advance the three major components of EarthMAP: (1) data and information integration, (2) integrated predictive science, and (3) actionable intelligence, delivered at the speed and scale of decision making. Overall, Use Cases are driven by the needs of stakeholders and serve to identify existing and future USGS (and partner) science capacity and technological innovations that will meet those needs. By co-developing Use Cases directly with stakeholders, the USGS will be better positioned to deliver science at the speed and scales that those stakeholders need.

The EarthMAP Use Case Development Team, formed by the Project Management Team and working with Regional Directors, is charged with leading the effort to create a set of clear and compelling Use Cases from each region. The Use Cases identify high priority decision-making needs relevant to our stakeholders that require the integrated science focus of EarthMAP. The first step is to conduct an internal round of Use Case identification, working with leaders and scientists in each region, to understand where we think our stakeholders will benefit from EarthMAP and continue to refine the EarthMAP vision. The second step is to establish a catalog of Use Case development opportunities coming from those efforts and select an appropriate subset for initial engagement with our external stakeholders and decision makers. The third step will involve stakeholder engagement through targeted workshops or other direct interaction to further explore the Use Case(s) and to identify additional needs that can only be addressed through a more fully developed EarthMAP.This document includes:

- Section 1: Guidance for Developing Use Cases

- Section 2: Use Case Summary Template

The guidance and template can be adapted by each region as necessary, but form the basis for (1) identifying high-priority regional and national Use Cases that reflect EarthMAP goals, desired outcomes, and products; (2) creating clear and compelling examples and goals that the USGS and its collaborators can achieve over the next decade(s) as we develop EarthMAP; (3) engaging both scientists and stakeholders in developing innovative approaches to accomplish EarthMAP goals; (4) building a shared sense of ownership and investment in the future of EarthMAP and a 21 st-century USGS; and (5) establishing and reinforcing relationships with key local, state, and regional stakeholders.

\section{Section 1: Guidance to Identify, Develop, and Prioritize EarthMAP Use Case Summaries}

The Use Case Development Team developed the following guidance to serve as a screening tool to help regional leaders identify high priority Use Cases. The Use Case summaries will serve as the first step in the review and prioritization of potential EarthMAP projects which could be explored further and in more detail. It is recommended that each USGS region aim to generate three to seven regional, multi-regional, or national Use Cases in the first step of this process through internal workshops, meetings, or other engagement with leaders and scientists within the region.

\section{Demand and Relevance}

Use Cases are intended to be "demand-driven"- that is, they describe scientific and decision problems that are of high interest to decision makers and stakeholders where we have existing USGS expertise and strong interest from our partners and where the elements of EarthMAP are necessary. Use Cases clearly define the problem to be addressed, the relevance of the problem as a societal issue, and who can benefit from additional science to address the problem(s). A key aspect of EarthMAP is that it is integrative by design, going beyond questions that traditional, single discipline science can answer. Use Cases will describe why this EarthMAP approach is necessary to advance and deliver USGS science to appropriately address the problem(s) of interest. Further, Use Cases will describe alignment with existing regional and partner or stakeholder priorities, extensibility in geographic scale, topical integration, and applicability for multiple decision support applications. 


\section{Improving Decision-Support}

EarthMAP is intended to improve the USGS capacity to deliver predictive information to decision makers and stakeholders through forward looking science and modern data integration, synthesis, and delivery approaches. Explicitly improving the decision-making process through access to appropriate earth science information is essential to the success of EarthMAP. Use Cases will identify what aspect of the decision-making process is targeted for improvement (for example, information accuracy and resolution, information scope and value, timeliness of delivery, accessibility and useability) and how that fits into decision and action frameworks. Use Cases will describe the co-development process and implementation approach of the resulting information product.

\section{Science Capabilities and Advancement Opportunities}

The Use Case will identify and describe current USGS expertise and data collection mechanisms while also defining opportunities for science advancement, new data needs, and integration. These opportunities can include interdisciplinary approaches and inter-regional collaboration and can improve partnerships with local, state, federal, non-profit, or other private entities. Overall, Use Case topics address science-informed decision making of substantial societal importance and demonstrably advance existing USGS monitoring, analysis, and predictive capacity to address our stakeholder's most pressing needs. Use Cases build upon existing data streams and research programs while also outlining how we can improve our ability to deliver actionable, integrated scientific information more accurately and quickly through partnership and stakeholder engagement.

\section{Operational Implementation and Technical Considerations}

The Use Case will identify challenges, including gaps in current capabilities, existing bottlenecks of data access, organization, integration, and delivery; it will describe implementation challenges given current and potential organizational capacity and partnerships. Opportunities to co-produce knowledge and technological processes through deliberate external partnerships in addressing the Use Case should be identified, along with existing and potential partners who can work with the USGS toward solutions for improved decision making. The need for any new metadata standards to support tracking of data provenance and lineage should be identified. The Use Case should align with current or potential computational resources to manage data production, processing, and delivery, or identify where partnerships could assist with such efforts.

\section{Longevity and Sustainability}

Finally, Use Case descriptions will address whether the earth science information product(s) have long-term viability and sustained relevance to identified stakeholders and opportunities to extend the work in a sustainable way to benefit other stakeholders. The Use Case will identify existing and needed requirements to attain long-term viability of the approach. The Use Case also will define the activities to be sustained throughout the life cycle of the decision/action framework as necessary to ensure continuity of decision support. Use Cases also describe transferability and scalability of deliverables across geographies and across disciplines, as well as to other applications.

\section{Section 2: EarthMAP Use Case Summary}

This template is intended to facilitate development and documentation of Use Case descriptions and is aligned with the guidance described earlier for identifying EarthMAP Use Cases. The summary should be concise, 4-pages (written) maximum (12-point font; 1-inch margins) in length, and can include a relevant figure, if appropriate. Any submission longer than 4 pages (not including the single figure) will not be considered.

Title of Use Case:

Region(s):

Scientific Discipline(s) Represented:

Primary Contact:

Email:

Executive Summary (one to two sentences)

1. Demand and Relevance - Define the overarching science and decision problem your Use Case is intending to address. Please identify the USGS Mission Areas, USGS programs, other USGS regions, and external partners that you are working with, or will be contacting to work with, on this Use Case. Additionally, specifically describe the stakeholder interest in this Use Case. Please consider the following points in your response:

a. Decision(s) this EarthMAP Use Case will inform.

b. Relevant decision makers, stakeholders, and intended information recipients; their interests in, and need for, improved science information.

c. Integrated science and information synthesis necessary to address the problem in context of EarthMAP and the decision(s) to be made. 
d. Relationship to known stakeholder priorities and to program, mission area, agency, bureau, departmental, and administration priorities.

e. How could meeting this demand lead to extended science and products that would address decision problems at different scales or periods?

2. Improved Decision-Support—Describe how your Use Case will improve stakeholder decision making. Please consider the following points in your response:

a. Aspects of the decision-making process that will be improved (for example, information accuracy and resolution, information scope and value, timeliness of delivery, accessibility, and useability).

b. Opportunities to work directly with decision makers to define their integrated science needs and understand and meet their needs for science delivery.

c. Expected impact of the improved decision or resulting management action.

\section{Science Capabilities and Advancement}

Opportunities-Describe the existing foundational data, science, model(s), and information technology to support this project and the new approaches, capabilities, technologies, and partnerships that must be developed to address decision-maker and stakeholder expectations. Please consider the following points in your response:

a. Existing scientific and technological capacity to support this project (internal and external).

b. Potential for integration with other USGS projects, accelerating delivery of actionable intelligence.

c. Existing USGS and partner datasets available for synthesis, or model integration.

d. Existing and potential partnerships to improve the science capability, data development, and timely delivery and close critical data gaps.

\section{Operational Implementation and Technical}

Considerations-Describe the existing and potential

USGS capability to support initiation of the Use Case as well as how external partnerships could be leveraged to make the Use Case operational. Please consider the following points in your response:

a. Current and potential technical capacity to execute the Use Case including internal and external capabilities - what is and what could be.

b. Current and potential approaches for science delivery necessary to meet the time and needs of existing decision or management-action frameworks.

c. Data storage, legacy data access, data lineage, and data delivery mechanisms needed to bring the Use Case to life.

d. Potential science and technology partners who can work with the USGS to deliver on the Use Case.

e. Additional resource needs - both internally and through partnerships - to address operational implementation, including computational resources and new expertise needed (for example, artificial intelligence/machine learning (AI/ML) experts, data scientists, engineers).

5. Longevity and Sustainability - Describe the long-term viability of the science and technologies that will be developed for the proposed Use Case. Please consider the following points in your response:

a. Anticipated long-term relevance of this Use Case.

b. Requirements to sustain this effort over the lifetime of the decision or management-action framework.

c. Transferability and scalability of deliverables across geographies and to other applications.

d. Information that will be used to evaluate the effectiveness of this effort and define metrics of performance and success.

e. What other problems, at different scales, different timeframes, and or in different geographies, will benefit from the science and technologies developed for this Use Case. 


\section{Appendix 2. Use Case Development by Region}

\section{Alaska Region}

The Alaska Regional strategy to develop first-round Use Case submissions focused upon leveraging previously acquired input from external partners and stakeholders toward the advancement of specific and feasible interdisciplinary goals that seemed most advantageous for implementation within a timeframe of $1-3$ years. Through a series of weekly virtual managerial meetings from June to August 2020, the Regional Office and Regional Center Directors reached consensus on a few select topics with potential for new investment in the context of EarthMAP goals and 21st-century technologies. The process was grounded by grassroots initiatives selectively filtered through management to assess the timeliness of the opportunity. The process also allowed for additional Use Case submissions to emerge through fall 2020, as managers continued to evaluate incoming guidance and determine which Use Cases to submit. The process yielded five Use Cases that are intended to become operationalized as resources become available. The Alaska Regional Office supported each of these Use Cases for some degree of implementation during fiscal year (FY) 2021. For example, in explicit support of EarthMAP, the Regional Office provided financial support to the University of Alaska Fairbanks to begin work on the Use Case entitled "Enhanced Delivery of Integrated Ecosystem Model Output" to increase USGS capacity to access and use downscaled climate projections, develop impact models at regional and local scales, and enable better stakeholder access to output products.

\section{Midcontinent Region}

The Midcontinent Region held a Science Chief Forum organized by a team that included three regional science coordinators, a representative from the Use Case Development Team, and one from the Capacities Assessment Team, working with the Regional Director and Deputy Director. Science Center Directors, Deputy Directors, Branch Chiefs, and leading Principal Investigators participated. Regional leadership introduced focal Use Case topics that included biosurveillance, harmful algal blooms, integrated water monitoring and prediction, and climate adaptation. Leadership determined that regional Use Cases be aimed toward basinwide and multi-basin applications, including the upper Mississippi River, Missouri River, and Great Lakes. Writing teams were comprised of Science Chiefs working collaboratively on Use Cases specifically aligned to regional priority topics defined by regional leadership and under consideration of the Regional Science Plan.

At the end of the introductory forum, regional leadership requested the development of teams of Science Chiefs to collaboratively write short - three to five sentence-Use Case summaries on the focal areas. To begin the process, Science Chiefs were asked to submit more refined ideas for Use Cases by focal topic identifying the problem, potential impact, the information user, and approach. These problem statements were submitted within a central document stored on a shared file directory for transparency. Science Chiefs were encouraged to comment within the documents to begin a discussion on these ideas. Subsequent review by Regional Science Coordinators led to five final topics selected as Use Case topics to be developed by the writing teams. A follow-up meeting with Science Chiefs focused on finding alignment with the five Use Case topics and the Use Case problem statements. Scientists were requested to join writing teams, facilitated by Regional Science Coordinators, to ensure drafts were completed. Regional leadership reviewed the drafts and worked with the teams to finalize. The resulting five Use Cases were submitted to the Use Case Development Team (UCDT). 


\section{Northeast Region}

The Northeast Region conducted a formal solicitation for Use Case proposals from the science staff of the 13 Science Centers. A total of eight proposals were submitted, including four for the Delaware River Basin (DRB). The DRB proposals were consolidated to build a broader integration plan. Each of the eight Use Cases included experiments with integrating science across disciplines, space, and time. A virtual leadership team meeting with Science Center Directors was held to discuss the Use Cases received, and seven Use Cases were selected for submission to the UCDT. Regional leadership determined that urban-coastal resiliency was identified as a topic of high regional importance in the Northeast Region's Use Case development process. A coastal resilience initiative design workshop provided an opportunity for Northeast Regional scientists to interact, build partnerships on urban/coastal science resiliency capabilities, and identify specific interdisciplinary studies that align with the integrated predictive science vision. Major issues in urban and coastal resilience, from a stakeholder perspective, were addressed, types of monitoring data and information available for the baseline analysis (as well as observational gaps) were described, and existing models for understanding change for the urban/coastal environment (and gaps in modeling capabilities) were documented. Following this workshop, the Region began collaboration discussions with the New York City (NYC) Mayor's Office of Resiliency, with the goal of building integrated groundwater, surface-water, and coastal surge models for forecasting compound flooding hazards in the NYC metropolitan area.

\section{Northwest-Pacific Islands Region}

\author{
The Northwest-Pacific Islands Region held a meeting \\ with Gary Rowe-EarthMAP Project Management Team \\ (PMT) Chair/Coordinator-and the Science Center leads in \\ March 2020. Regional leadership asked the Science Centers \\ to provide information on EarthMAP capabilities that could \\ support Use Cases during Science Center reviews in March \\ and April. Guidance was sought from the Northwest Climate \\ Adaptation Science Center, which had been engaged with \\ numerous external stakeholders over the last 2 years, to
}

identify Use Cases aligned with EarthMAP. In August, a regional meeting was held to discuss potential Use Case topics and identify Use Case submission timelines and internal regional reviewers. The Northwest team, comprised of mid-career scientists (Multidisciplinary Science Leadership team or MUSCLE), met in late September to explore interdisciplinary science topics for consideration as well. In October, scientists, Center Directors, and senior principal investigators, along with the MUSCLE team, presented final Use Cases to the Region. Upon review, the Region put forth five final Use Cases.

\section{Southeast Region}

The Southeast Region's approach to Use Case development was to use its annual, 3-day Science Workshop as a platform for the EarthMAP PMT to present the vision and engage scientists in exploring the EarthMAP concept. The workshop was held on July 29-31, 2020, as an all-virtual event with the main goals of (1) engaging attendees in exploration and discussion of the EarthMAP concept, (2) providing input to EarthMAP capacity assessments, and (3) developing Use Cases to inform EarthMAP planning. The workshop agenda featured a mix of plenary sessions, lightning talks offering potential Use Cases and describing existing regional capacities, and breakout sessions that delved deeper into each of these topics. The following Use Cases were identified:

1. Addressing changes in sediment transport, coastal erosion, and barrier island evolution

2. Advanced monitoring and modeling of coastal salinity

3. Advanced monitoring and modeling techniques to assess water quantity and quality change in the Mississippi Alluvial Plain, under a variety of use and optimization scenarios.

A post-meeting survey revealed a significant percentage of the attendees who indicated that they have a better understanding of the EarthMAP concept, the differences between capacities and Use Cases, and the many ways their skills and projects can contribute to EarthMAP. After review from staff, Center Directors, and the Regional Director, the Region put forth two Use Cases for consideration. 


\section{Southwest Region}

The Southwest Region's approach to Use Case development was driven by regional leadership: to ensure alignment with top science priorities that represent the Region's unique science challenges. The Region's leadership began communicating and distributing materials for the development of the Use Cases in late summer 2020. At that time, the Regional Director requested that the priority focus would be on the development of a Wildfire Use Case and associated sideboards related to wildland fire science. Additional topical Use Cases would also be considered, which met the Use Case criteria and highlighted regional science capacity and expertise scalable to other regions and nationally. There was an internal regional meeting with Science Coordinators, Center Directors, and Principal Investigators (PI's) on September 9, 2020, to discuss the EarthMAP Use Case development guidance documents with the UCDT Region Lead presenting the UCDT goals and objectives. The Southwest Region circulated the Use Case Development Summary Guidance and Template documentation and request for submissions in early fall. A total of five Use Cases were developed in this effort.

\section{Rocky Mountain Region}

The Rocky Mountain Region (RMR) held a 3-day virtual Science Exchange Workshop in mid-September 2020, with a focus on EarthMAP and drought in the Colorado River Basin (CRB). A half-day session was devoted to the EarthMAP Colorado River Basin Pilot, which included 18 breakout sessions to brainstorm and discuss Use Case ideas. The workshop was held in cooperation with the Southwest Region because much of the CRB is in the Southwest Region. The workshop was well-attended; more than 300 people registered for the meeting, with up to 200 participants per day. Each workshop session explored how current science projects could support EarthMAP. The week following the workshop, the RMR circulated a request to the Regional Science Center Directors for Use Case submissions that would support the CRB Pilot and RMR science priorities. The request asked that Use Cases be submitted in 4-weeks' time, using the criteria and template developed by the Use Case Development Team. The RMR Director's Office hosted a question-and-answer session to address questions and provide additional guidance on the Use Case submission process 2 weeks after the initial request. The RMR Science Centers submitted seven Use Cases to the Region.

Additional regional Use Case Activities included:

- An internal USGS Need-to-Know article on the RMR Science Exchange meeting was released on September 28, 2020.

- A post-meeting on-line survey was circulated to obtain impressions, suggestions, comments, and input from workshop attendees.

- A RMR Science Exchange website has been developed, containing the meeting Program and links to a digital repository that hosts copies of the meeting presentations, abstracts, summary documents, recordings of the presentations, and panel and question and answer sessions. USGS Rocky Mountain Region Science Exchange-Home (sharepoint.com).

- A report summarizing the RMR Science Exchange meeting objectives, panel session outcomes, recommendations, and polling results is expected to be completed by the end of fiscal year 2021 .

- The RMR began a monthly Science Seminar series focusing on EarthMAP.

- An internal USGS Need-to-Know Blog: EarthMAP introducing the Pilot Planning Team was released November 9, 2020. 


\section{Appendix 3. EarthMAP Use Case Characterization Form}

\section{EARTHMAP Use Case Characterization Form}

1) Use Case Title:

2) Region of submission:

3) Other USGS Regions Directly Identified:

4) Mission areas Involved:

5) Dominant topical themes (e.g. hazards, coastal change, water availability, invasive species):

6) Major disciplines involved from the USGS Circular 1309 "layer cake"(Check all that apply):

Geology
Soils
Land Surface
Surface/Groundwater

Geology

Land Surface

Surface/Groundwate
Flora

Fauna

Humans

Atmospheric science

7) EarthMAP concept alignment (Check all that apply):

Integrate data/observations

Modeling/prediction

Improved information delivery

8) Extent of external stakeholder planning network (Numeric entry; see definitions below):

a. Total Number External Stakeholders Identified:

b. Additional Comments (e.g., level of current engagement, organizational level):

9) Degree of detail/specificity in decision being informed: Low_ Medium High

Low: Decision being informed is not identified or too broadly generalized (e.g., "useful for managers to make decisions")

Medium: Specific decisions and decision makers were identified;

High: Specific decisions and decisionmakers were identified and described how resulting scientific information will fit into the decision framework.

10) Aspect of decision process being improved (Select Yes/No for each):
a. Information accuracy and resolution
Yes/No
b. Information scope and value
Yes/No
c. Timeliness of delivery
Yes/No
d. Accessibility and usability
Yes/No

Figure 3.1. EarthMAP Use Case Characterization Form 
11) Degree of detail/specificity in "advancement opportunity": Low_ Medium High

Low: Advancement opportunity not clear or lacking

Medium: Advancement opportunity identified but pathway towards that opportunity unclear; or gaps in logic.

High: Advancement opportunity identified, and pathway clearly described with clear logical linkages.

12) Notable criterion explicitly addressed by submission (Check where adequately addressed):

Demand and relevance Improving decision support

Science capabilities and advancement opportunities

Operational implementation Longevity and sustainability

13) Degree of aspiration beyond current norms of action: Low Medium High

Low: likely to happen without intervention

Medium: some capabilities need to be developed but can be done in a reasonable time frame

High: major effort needed to develop capacities or partnerships to complete

14) Degree of feasibility: Low Medium High

Low: major effort will be needed over extended period to develop capacities to complete Medium: some capabilities need development, but likely to occur with modest effort in modest timeframe

High: Minor effort required, most capabilities already developed, relatively short time frame to implement

15) Scalability: Local Regional Sub-national National

16) Degree of stakeholder engagement already underway: None Low Medium High

Low: some partnership arrangements already in place

Medium: substantial partnerships arrangements already in place

High: partnerships and planning workshops already established and ongoing

17) Reviewer estimation of Implementation timeframe for entire Use Case:

Less than 3 years Greater than 3 years

18) Execution challenges: Low Medium High

Low: capacity and partnerships and planning workshops already established and ongoing Medium: substantial capacity/partnership arrangements already in place

High: some capacity/partnership arrangements already in place

Figure 3.1.-Continued 
19) Major impediments (Check all that apply):
Cost Capability Gaps
Personnel
Prioritization
Other

20) Any additional notable observations:

21) PARKING LOT - What questions or pieces of information did we neglect to ask in the Use Case Submission template?

22) Summary Characterization (Check ONE; see broad definitions below):

Use Case Capacity Building Aspirational

\section{Use Case:}

$\otimes \quad$ Identifies high-priority science applications that advance the three major components of EarthMAP: 1) data and information integration, 2) integrated predictive science, and 3) actionable intelligence, delivered at the speed and scale of decision making.

$\otimes$ Demand-driven by decision-makers and stakeholders (eg, they describe scientific issues and problems that are of high interest to decision-makers and stakeholders, for which the USGS has expertise and our partners have expressed strong interest, and that require the capabilities of EarthMAP to address.)

Q Helps identify existing and future USGS and partner science capacity and technological innovations that will meet those needs.

\section{Capacity Building}

Q Developing/promoting our people and their associated skill sets, technical and (or) scientific expertise, and experience conducting integrated science projects.

$\otimes$ Developing USGS monitoring networks, current (and historic) data, models, analytical and visualization tools, information technology and management capabilities, and other scientific products that support one or more of the three EarthMAP components: 1) data and information integration, 2) integrated predictive science, and 3) actionable intelligence

\section{Too Aspirational}

Q High risk, but also could be of high reward if it came to fruition

$\nabla$ Capability / capacity do not currently exist to make it happen in the near future (but could happen in $10+$ years)

Q There are not enough partners to currently demand this (not an immediate need)

$\nabla$ Requires too many resources and changes to make it happen

\ A "home run" and not only a first base

Figure 3.1.-Continued 


\section{Appendix 4. Use Case Author Capacity Assessment Survey}

\section{EarthMAP Use Case Capacities Summary}

This is a supplemental survey about your EarthMAP Use Case capacity and capability needs. The survey presents checklists of scientific and technological capacities and areas of expertise and asks you to indicate if you think sufficient capacities currently exist at the level necessary to support your Use Case. The three checklists reflect the three main components of EarthMAP and mirror the questions asked in the capacity assessment survey. Instructions: Please coordinate one response per Use Case. The survey should represent the assessment of the authors on whether or not existing capacity is sufficient to meet the needs expressed in the Use Case. Please indicate if the capability is not applicable to the Use Case. We limited the capability lists to those identified in the initial Capacity Assessment Team survey to preserve a direct link. If important capabilities and capacities needed for the Use Case are not included in the capability list, please describe those following one of the three sections, or in the general feedback question at the end. Thank you for taking the time to help us better understand the capacity requirements aligned to Use Cases and make a clear connection between the capacities assessed in the USGS-wide survey and the capacities that would be needed to support the Use Cases we received.

1. Title of the Use Case

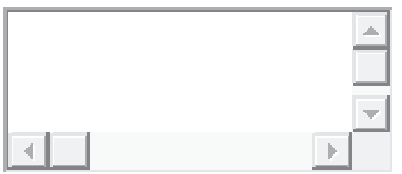

2. Data and Information Integration Capacity: For each capability listed below, identify if: (a) existing capacity is sufficient to meet needs identified in the use case, or (b) the capacity identified in the use case does not exist (or does not exist at the level necessary to support the use case)

\footnotetext{
a. b. Not Not

SufficientSufficientApplicab
}

Plane- and/or satellite-based imagery

Lidar

Unmanned Autonomous Vehicles/Drones

Biosensors

Hydroacoustic sensors
D D $\quad$ D

D $\quad$ D $\quad$ D

D D D

D $\quad$ D $\quad$ D

D D D

Figure 4.1. EarthMAP Use Case Capacities Summary Survey 


Microservers (wireless sensor network nodes), low cost trustworthy sensor networks
in situ fluorometry

3. Data and Information Integration Capacity: For each capability listed below, identify if: (a) existing capacity is sufficient to meet needs identified in the use case, or (b) the capacity identified in the use case does not exist (or does not exist at the level necessary to support the use case)

a. b. Not Not SufficientSufficientApplicab

Citizen science

D D D

Traditional Ecological Knowledge (knowledge acquired by indigenous or local peoples)

Figure 4.1.-Continued 


Volunteered Geographic Information (geospatial content generated by nonprofessionals
using mapping systems available on the internet, including crowdsourcing and social media)
$\begin{aligned} & \text { Data collected without attribution (for example, data on population movements to better } \\ & \text { understand exposure and vulnerability and better target communications) }\end{aligned}$
$\begin{aligned} & \text { Enhanced existing or developed new approaches for integration of large datasets } \\ & \text { Enhanced the extensibility of research and monitoring insights across spatial and/or temporal } \\ & \text { scales }\end{aligned}$

4. List any capacities related to Data and Information Integration that are not listed above that are needed for your use case, and indicate if they currently exist or need to be developed.

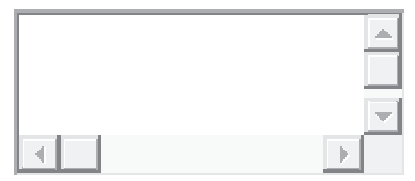

5. Predictive Science Capacity: For each capability listed below, identify if: (a) existing capacity is sufficient to meet needs identified in the use case, or (b) the capacity identified in the use case does not exist (or does not exist at the level necessary to support the use case)

a. b. Not Not SufficientSufficientApplicab

Bayesian network analysis

D D D

Statistical emulators (i.e. statistical models that mimic the outputs of a complex physical or simulated system for a set of inputs)

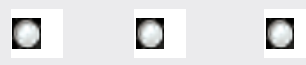

Figure 4.1.-Continued 


Phenomenological models
Artificial Intelligence/Machine Learning approaches
$\begin{aligned} & \text { Model-data fusion (approaches to improve model performance by either optimizing values of } \\ & \text { unknown parameters and initial conditions or by constraining the model by data) }\end{aligned}$
$\begin{aligned} & \text { Agent-based modeling (simulating interactions of autonomous "agents" representing } \\ & \text { individual organisms, with other agents and the external environment }\end{aligned}$
$\begin{aligned} & \text { Coupling and/or integration of multiple models } \\ & \text { Uncertainty estimates applicable to decision making }\end{aligned}$
Operational ecological forecasts useful to management decisions

6. List any capacities related to Predictive Science that are not listed above that are needed for your use case, and indicate if they currently exist or need to be developed. If there are other modeling approaches or methods that were not listed above, please list those below along with sufficient or not sufficient capacity indication.

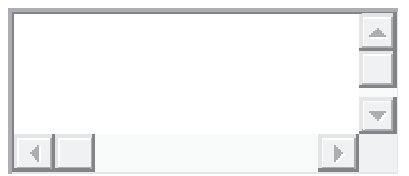

7. Actionable Information Capacity: For each capability listed below, identify if: (a) existing capacity is sufficient to meet needs identified in the use case, or (b) the capacity identified in the use case does not exist (or does not exist at the level necessary to support the use case)

a. b. Not Not

SufficientSufficientApplicab

Skills and experience in engaging with stakeholders

Skills and experience in co-development of projects with stakeholders

D $\quad$ D $\quad$ D

D D D

Figure 4.1.-Continued 
a. b. Not Not SufficientSufficientApplicab

Skills and experience with decision science (techniques used to inform decision making at various spatial, temporal, population, and behavioral scales), decision analysis, or structured decision making

Skills and experience to deliver data applications and visualizations

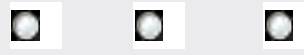

8. List any capacities related to Actionable Information that are not listed above that that are needed for your use case, and indicate if they currently exist or need to be developed.

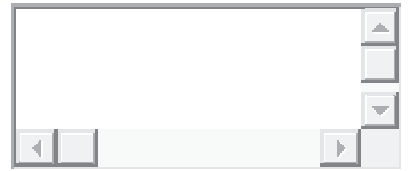

9.Now that you have contributed participated in developing a use case and contributed to the capacity assessment survey, do you have any additional information or feedback that you would like to share with the EarthMAP teams? (examples: Are there capacities that we overlooked? Are there improvements we can make to the use case development process?)

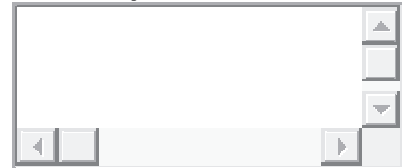

This content is created by the owner of the form. The data you submit will be sent to the form owner. Microsoft is not responsible for the privacy or security practices of its customers, including those of this form owner. Never give out your password.

Powered by Microsoft Forms

|

Privacy and cookies

| Terms of use

Figure 4.1.-Continued 


\section{Appendix 5. Regional Use Case Submissions}

Disclaimer: Uses Cases are reproduced here as submitted to the Use Case Development Team and have been minimally edited for style or content.

Region-led Use Case development resulted in a suite of 36 different Use Case submissions across the 7 USGS regions. Each Use Case is included in this appendix to showcase a potential portfolio of future integrated and predictive science capacity of the U.S. Geological Survey (USGS). These forward-thinking scientific approaches can be utilized as part of a "bottom-up" science strategy framework in coming decades during strategic planning activities. Use Case authorship is not detailed because many authors contributed; however, a primary contact person is included for each.

\section{Alaska Region}

\section{Machine Learning Analysis of Near Real-time Imagery of U.S. Volcanoes}

The USGS Volcano Science Center (VSC), the Federal Aviation Administration (FAA), and other partner agencies operate more than 50 telemetered cameras on or near dozens of active volcanoes throughout the United States. Ranging from the Western Aleutian Islands to Hawaii to Yellowstone, the ad hoc network of cameras spans a huge area more than $6000 \mathrm{~km}$ across. Imagery from the cameras are used primarily for the real-time assessment of local conditions, with the particular parameters of interest varying by user. The VSC uses imagery to detect and assess anomalous volcanic activity, typically during routine daily monitoring. After-the-fact studies are also common, as are impromptu checks to confirm (or reject) indications of volcanic unrest suggested by other data sources.

Over the last year, the VSC and the USGS Cloud Hosting Solutions Team (CHS) have developed a cloud-based system for collecting and storing imagery from the camera network. In addition to improving reliability, saving time, and lowering costs, the system also allows for easy and efficient access to Bureau cloud computing resources. At present, the system retrieves and stores more than 20,000 images per day.

To build on this success, we propose another collaborative partnership between VSC and CHS: the development of Machine Learning (ML) algorithms for the detection and classification of potentially significant volcanic events in camera imagery. Once complete, the algorithms will be incorporated into the VSC's existing notification infrastructure. Ideally, this work will be the first step toward a holistic, multi-parametric alarm system capable of detecting coherent patterns that manifest across disparate datasets.

\section{Demand and Relevance}

In 2005, USGS published the results from a systematic study of the Nation's potentially active volcanoes in a report called "An assessment of volcanic threat and monitoring capabilities in the United States: Framework for a National Volcano Early Warning System" (Ewert and others, 2005). Updated in 2018 (Ewert and others, 2018), the "NVEWS" assessment determined that the Nation's volcano monitoring infrastructure is, in the aggregate, about 25 percent of what is needed. Closing this monitoring gap is one of the VSC's top priorities. We view the widespread adoption of cloud services and machine learning technology as an effective and relatively inexpensive way to do more with what we already have, while also preparing us for the likely future growth of the Nation's volcano monitoring infrastructure.

Nearly all of the volcano imagery collected and (or) aggregated by the VSC is available to the general public with little or no latency. A variety of external users take advantage of this capability for a number of different purposes, including scientific research, meteorology, search and rescue, outdoor recreation, and aviation. The VSC's investment in cloud and machine learning technology for the aggregation, analysis, and distribution of volcano imagery will thus serve not only its own needs, but also the larger community of potential end users.

\section{Improved Decision Support}

Emergency management officials and the general public rely on the USGS for prompt and accurate forecasts of volcanic activity. Instrumental data such as those from seismometers or gas sensors can be ambiguous, especially if the number of instruments is small. Real-time imagery of a volcano's summit can, in many cases quickly resolve such ambiguities and thus improve both the timeliness and accuracy of forecasts and warnings.

At present, VSC personnel manually assess individual images for their potential volcanological significance, usually during routinely scheduled daily checks. The gaps between checks are sufficiently large (several hours) that in some cases the onset of a serious change in activity isn't detected until well after the fact. By flagging sudden or otherwise remarkable changes, continuous algorithmic assessment of incoming imagery has the potential to reduce the latency between the onset of unusual volcanic activity and its detection. Once proven, such technology could also relieve the monotony of regularly scheduled checks, thereby improving employee morale and efficiency while also freeing up time for more interesting and productive endeavors. 
Another potential benefit of algorithm assessment is the ability to identify and tag notable characteristics within images so they can be easily searched and retrieved at a later date. In principle, once such algorithms have been trained, they could be used on historical imagery, thus greatly improving the accessibility and usefulness of existing photo archives.

\section{State of the Science and Advancement Opportunities}

The proposed project leverages two existing resources: (1) the VSC's 15 years of experience operating telemetered cameras on volcanoes, and (2) the FY2020 collaboration between VSC/CHS to build a cloud-based system for collecting and storing camera imagery. Thus, the project's primary technical and scientific challenges consist of designing and testing a suite of machine learning algorithms that can scan through a series of images in real-time to identify scenes of potential interest. Although many image classification algorithms have been described in the literature and are in use operationally, the proposed project's functional requirements are sufficiently complex and novel, that an "off-the-shelf" solution is unlikely.

Most machine learning algorithms must be trained on existing datasets, and this can be a time-consuming process that often involves considerable human interaction. An additional complication is that cameras are deployed in a variety of environments ranging from arctic tundra to tropical rainforests - we may discover that each locality needs a separate training process.

\section{Operational Implementation and Technical Considerations}

The VSC already has a mature and comprehensive volcano monitoring capability spanning four major facilities in Alaska, California, Hawaii, and Washington. Integrating the results from the proposed project into our existing operational environment should be straightforward, with one possible exception: we may need to hire a new computer scientist to maintain and operate machine learning algorithms.

\section{Longevity and Sustainability}

The VSC had been sustaining its own system of data acquisition, storage and analysis for decades, thus we already have the system administrators and other information technology (IT) specialists necessary to keep the system running. We also have sufficient scientific expertise for the timely analysis and interpretation of incoming data required to fulfill our volcano monitoring mission. Thus, if the proposed project is successful, the longevity and sustainability of continuing its operation will not be in doubt. The uncertainty lies in whether or not the project succeeds, and if so, to what degree.

Primary Contact: Peter Cervelli, pcervelli@usgs.gov

\section{Building an Integrated Coastal Hazard Assessment and Mitigation Strategy with Bering Sea Communities of Alaska}

Every shoreline in America is vulnerable to extreme storm events that threaten lives and property, destroy infrastructure, disrupt local economies, and damage the environment. But due to diminished sea ice, changing wind patterns, erosion and rising sea levels, the dangers have become especially acute for Bering Sea communities along the north and west coast of Alaska, where coastal flooding hazards consistently demand attention as a top priority for regional adaptation and resilience planning efforts (fig. 5.1).

This Use Case aims to promote key steps necessary to advance the integration of relevant multidisciplinary observations with flood modeling and infrastructure mapping to produce new online risk assessment tools that inform local community planning and improve science collaboration among Federal, state, and regional partners for enhanced pre-storm preparations and post-storm recovery. By expanding coastal data collection and integration to deliver priority geospatial products, the broad collaborative effort in Alaska may yield a path of stakeholder service delivery that can be applied to other vulnerable regions of the nation.

\section{Demand and Relevance}

Coastal villages on the north and western shores of Alaska consistently identify declining sea ice and the corresponding increase in severe storm flooding as the most significant risk to infrastructure and the long-term sustainability of their community. At least 60 of Alaska's 144 threatened communities are located immediately adjacent to the ocean and are frequently impacted by Arctic storms and extra-tropical cyclones. The economic challenge to prevent failure of existing infrastructure in these communities is prohibitively high at an estimated cost exceeding $\$ 4.3$ billion over several decades. Concurrently, these communities face multiple barriers that make potential mitigation strategies even more challenging than in the contiguous United States, including: small population densities with no road system, no tax base, and limited safety and public health services. 


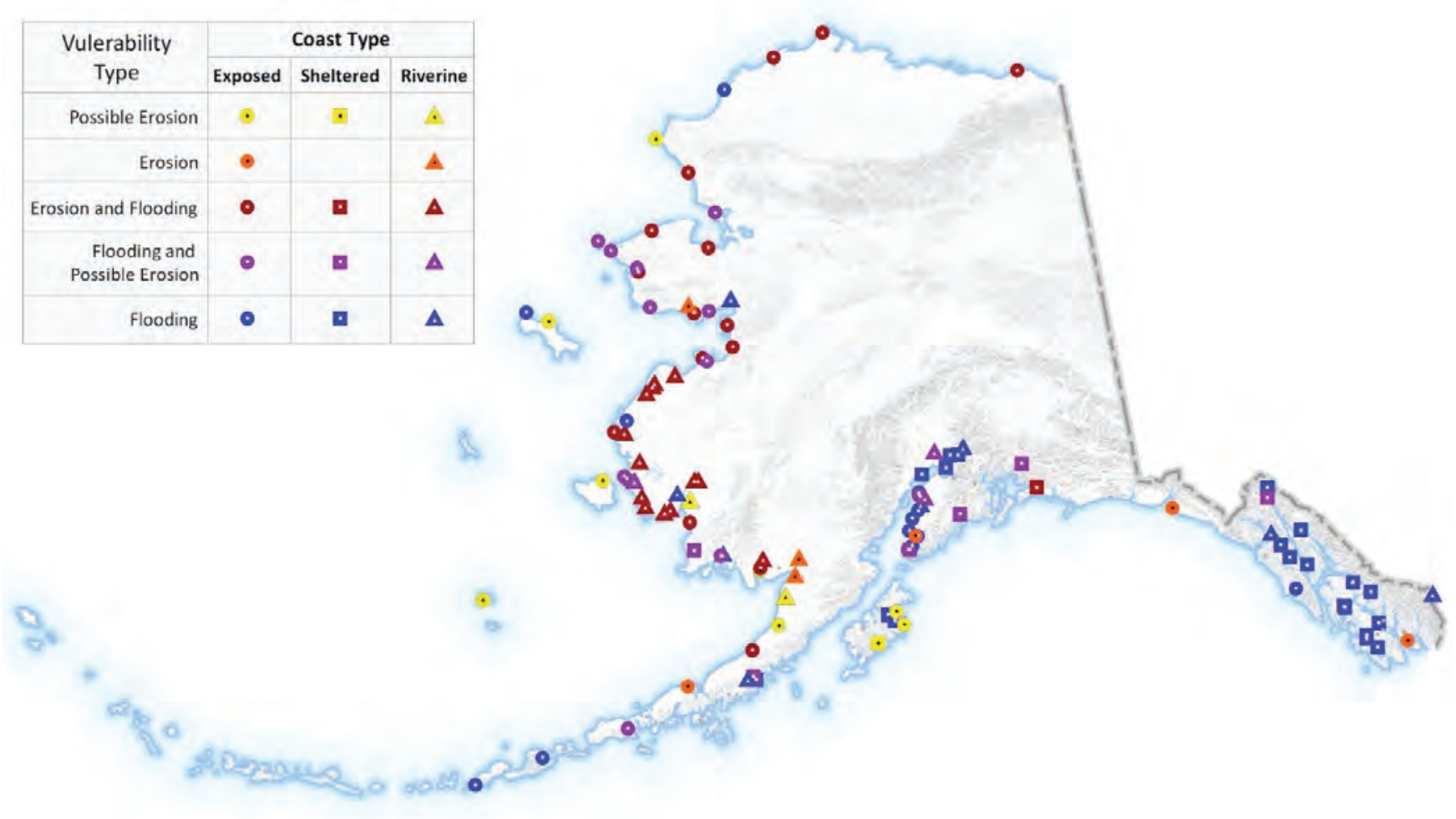

Figure 5.1. Coastal flooding and erosion. Credit: Alaska Division of Geological and Geophysical Surveys.

In the absence of expensive engineering solutions, Alaska needs an expanded scientific plan of collaboration to pursue increasingly urgent priorities in addressing the overwhelming magnitude of this problem. The scope of work transcends the purview of any single agency, disciplinary focus, data stewardship or trust responsibility. The proposed Use Case therefore seeks to strategically integrate the most urgent data, forecast delivery, and impact mitigation services needed from partner agencies such as USGS, National Oceanic and Atmospheric Administration (NOAA) Office of Coastal Management, NOAA National Weather Service, Alaska Ocean Observing System (AOOS), Bureau of Indian Affairs (BIA), U.S. Army Corps of Engineers (USACE), the Denali Commission, the State of Alaska Coastal Hazards Program, and the Alaska Native Tribal Health Consortium. Anchorage offers a promising location to reinvigorate this effort of national significance because of the shared administrative space of these multiple institutions within a common geography and jurisdictional authority.

The collaborations identified here directly advance the goals of the 2020 Alaska Coastal Mapping Strategy (following a 2019 Presidential Memorandum), whose implementation plan is currently under development. Likewise, this Use Case directly advances data gaps already identified by the
Alaska Water Level Watch, which aims to expand water level observations necessary for flood modeling and storm surge forecasting. The Use Case is also well timed to leverage upcoming workshop discussions at the 3rd Alaska Coastal Mapping Summit on December 9-10, 2020, jointly sponsored by the Alaska Department of Natural Resources, USGS, NOAA, and AOOS.

\section{Improved Decision-Support}

This Use Case seeks to improve decision support in multiple ways. One line of effort will involve accelerating installation of water level sensors and monitoring equipment for all high-priority coastal communities, ports and harbors to comparable degrees of coverage. Accurate water level and ice observations are basic building blocks for storm-surge forecasting and informed emergency response, yet the conventional technologies are not well suited to the remote and low-infrastructure conditions of Alaska. NOAA is actively investing in a range of technologies and community training activities to fill the gaps in water level and ice observations across the state. More investment is needed both to accelerate the coverage and to standardize the data. 
Another line of effort will involve completion of shoreline erosion assessment of both historical rates and future projections. The USGS Pacific and Marine Coastal Science Center has completed most of the historical work in Alaska, but projecting near-term shoreline change requires more attention. The USGS Coastal Storm Modeling System $(\mathrm{CoSMoS})$ is a dynamic modeling approach recently developed to allow more detailed predictions of storm induced coastal flooding, erosion, and cliff failures over large geographic scales. CoSMoS projections are currently available for regions in California, but have not yet been applied to conditions in Alaska.

A third major line of effort will refine stakeholder mapping priorities, costs, data standards and integrate and upgrade nearshore bathymetric and topographic data at the coast. Improved knowledge of precise beach elevation through strategic deployment of tide stations and bathymetric LiDAR acquisition will also yield more accurate erosion measurements and storm surge estimates. All of these lines of effort will be necessary to develop highly useful end products of coastal storm inundation risk assessment maps. These derived new products can then inform and dramatically improve the needed community planning and development of timely mitigation solutions.

\section{State of the Science and Advancement Opportunities}

The USGS/NOAA Total Water Level and Coastal Change forecasts and the USGS Coastal Change Hazards Portal are the only national scale products for assessing coastal-change impacts of storms on coastal environments. While these state-of-the-art products begin to address a pressing national demand for coastal hazards information in some locations, they are not yet able to reliably and efficiently provide agile delivery consistent with user requirements. For example, users such as NOAA National Weather Service (NWS), National Park Service (NPS), USGS, and local emergency management officials depend on our existing storm forecast products. However, the USGS does not presently have the capability to rapidly update coastal mapping data and ingest real-time observations to provide the best-possible products. The accuracy of the forecast as well as the utility of assessments may be degraded by out-of-date information. The situation is even more patchy and underserved in low-infrastructure locations such as rural Alaska, where significant data gaps still exist.

\section{Operational Implementation and Technical Considerations}

Coastal flooding forecasts are currently only available at a regional level and only take into account storm surge. Local forecasts are needed at individual communities to effectively respond to and avoid impacts to people and infrastructure.
Storm forecasts must also include wave run-up to predict total flooding. Anticipating flood impacts requires combining multiple baseline datasets to convert modeled storm water levels to local elevations. In order to plan for where the coast will be or how high floods will reach, long-term models of flooding and erosion are necessary which tie together all the near-shore and marine processes that affect coastal landforms. With further refinements, the conceptual model can be used to identify vulnerable locations and weather events to assess relative vulnerabilities of specific coastal facilities and systems. This information feeds directly into climate change adaptation planning, community infrastructure placement, and engineering projects in each nearshore community.

\section{Longevity and Sustainability}

Alaska's extensive shorelines are incompletely mapped and poorly monitored for the evaluation of coastal dynamics. Improved coastal mapping data are critical for a multitude of reasons, including informed management of coastal lands and Alaska Native trust resources; safe navigation in an ice-diminished Arctic; and responsible resource and energy exploration. Community resilience to coastal hazards such as flooding, erosion, and tsunami begins with integrated mapping data to establish baseline conditions and forecast change. With renewed focus and funding resources likely to be associated with the Alaska Coastal Mapping Strategy, Alaska's Federal, State, regional, tribal, academic and private sector partners have the opportunity and capacity to secure a targeted, collaborative, and innovative approach to comprehensive coastal mapping. The return on investment is apparent with the vast potential for improvements to life, safety, and economic opportunities that will accrue to Alaska and the Nation from a solid coastal mapping foundation. With corresponding targeted new investments, USGS could transform this Alaska-based Use Case into a pioneering demonstration of coastal hazard forecast delivery for application at a national scale.

Primary Contact: Dee Williams, dmwilliams@usgs.gov

\section{Wildlife Tracking Portal to Improve Data Documentation, Delivery, and Analysis}

The USGS Alaska Science Center (ASC) will expand the USGS Alaska Science Center Wildlife Tracking Data Collection (WTDC) to provide data management, metadata, and delivery of wildlife tracking data collected from satellite tags. Satellite tags are commonly used to track a variety of terrestrial wildlife species including birds and mammals. The ASC has developed an automated process for data management and delivery through its Trusted Data Repository and by extending this service to all USGS scientists (and others) using satellite tags will increase efficiency of data collection and distribution and will create a single and uniform data source for analyzing wildlife movements within the EarthMAP analysis environment. 


\section{Demand and Relevance}

Understanding the short- and long-distance movements of wildlife is critical for a wide variety of ecological research questions and management decisions. Tagging and tracking of animals is often used to determine locations of animals throughout their annual cycles, understand patterns of habitat use, quantify time spent on certain behaviors, and identify geographic areas repeatedly used by wildlife that may indicate sites of importance to species and populations. Satellite tags offer the ability to monitor movements and locations of animals across vast landscapes, across international boundaries, and throughout all seasons of the year. Satellite tags are used by scientists across throughout USGS and partner agencies and data management and documentation is complicated and data are not currently served through a unified portal. Expanding use of the ASC WTDC will increase efficiency and service of wildlife tracking data used by management agencies such as U.S. Fish and Wildlife Service
(FWS), Bureau of Land Management (BLM), Bureau of Ocean Energy Management (BOEM), National Park Service (NPS), U.S. Forest Service (USFS), and state and local agencies to make land and resource management decisions and demonstrate responsive action to Secretarial Order 3362.

\section{Improved Decision-Support}

Wildlife tracking data collected using satellite reporting tags are either distributed through a range of different and unconnected data servers or are not available due to lack of resources to manage, document, and serve these complex and intensive data. Expanding use of the ASC WTDC will allow for improved opportunities to serve data and provide data for a range of analyses including habitat use, distribution, and the timing of migration for use by scientists, managers, and the public. The ASC WTDC currently serves data for eighteen species of birds and mammals (fig. 5.2).

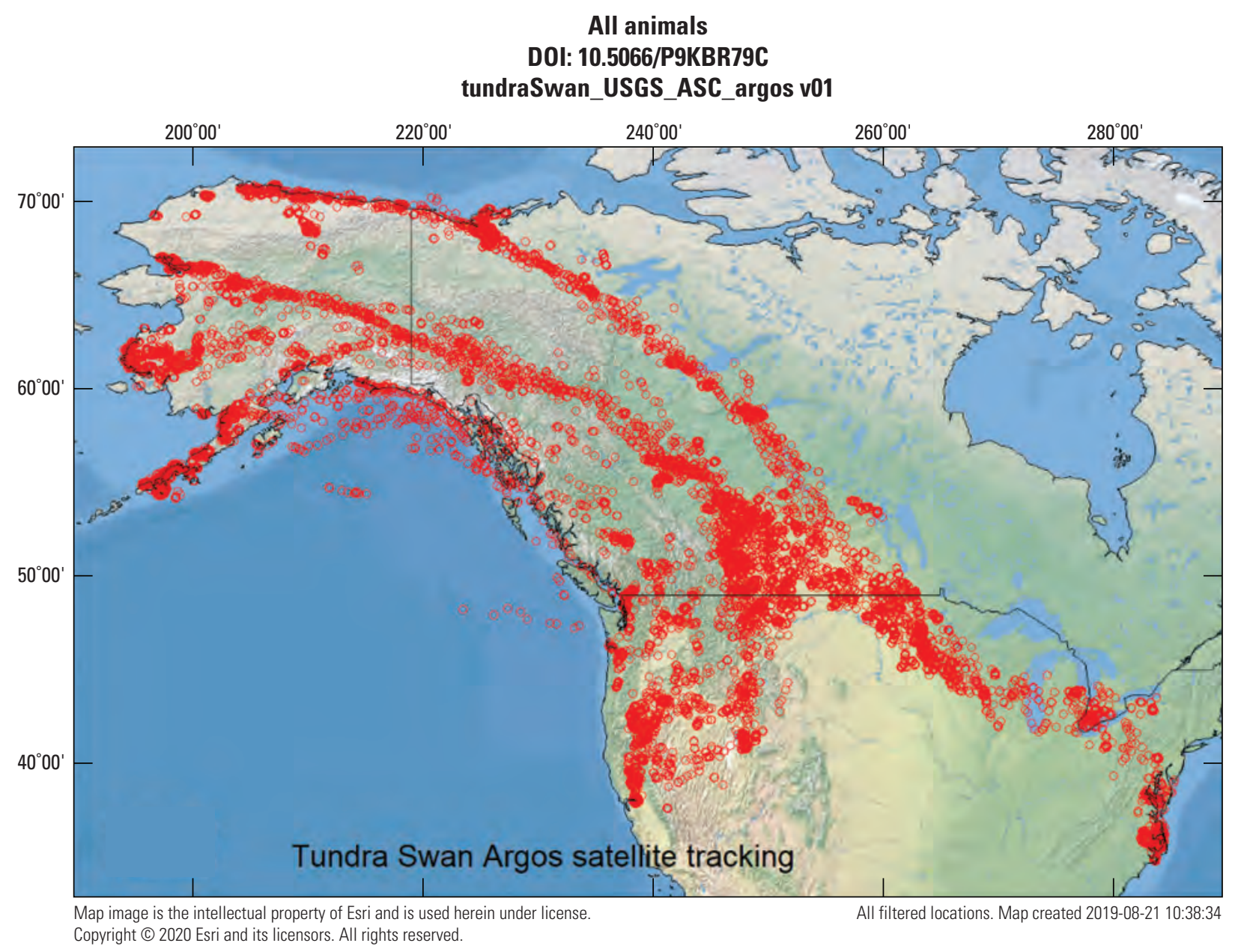

Figure 5.2. Example of data served by the Alaska Science Center (ASC) Wildlife Tracking Data Collection (WTDC). 


\section{State of the Science and Advancement Opportunities}

Satellite tagging is a mature method of tracking wildlife and has been used broadly to track movements of a range of species. Data management and delivery, however, is not consistent among users of these tags. As a result, it is difficult to summarize data or conduct further analyses using these data. The ASC has developed a workflow to ease the burden of data documentation (metadata) and delivery of satellite tagging data. Expanding use of the ASC WTDC will provide benefits to both scientist and users of these data.

\section{Operational Implementation and Technical Considerations}

The ASC will provide data management and service for all USGS and Department of the Interior (DOI) scientist using satellite tags through the ASC WTDC. Working with Core Science Systems, ASC will seek to serve these data through the Cloud so that they are broadly available for analysis in an EarthMAP analysis environment.

\section{Longevity and Sustainability}

The ASC is a trusted data repository and will continue to serve data in perpetuity. By expanding this service to wildlife researchers across the USGS and DOI, ASC will commit to ensuring these data are continually served as data and computing platforms evolve.

Primary Contact: Christian Zimmerman, czimmerman@usgs.gov

\section{Customized Arctic Map Products for State, Federal, and International Use}

The USGS Alaska Regional Office, in coordination with the National Geospatial Technical Operations Center, is responding to Federal and State government expressions of urgent need for updated U.S. Arctic Research Policy Act (ARPA) Boundary maps that provide value-added attributes for both marine and terrestrial domains as a second generation modification to the Boundary shapefile and map originally formatted for the US Arctic Research Commission and the National Science Foundation in 2009 (see www.arctic.gov/maps.html). The fundamental rationale for the USGS update is the increasing relevance of Arctic issues to national and global affairs that requires more functional projections and the creation of an interactive web application allowing users to interact with the maps in a dynamic up-to-date environment.

\section{Demand and Relevance}

The increasing relevance of Arctic issues to national and global affairs requires a more professional and functional set of maps and shapefiles than was created over a decade ago as an interim representation of significant geospatial and political boundaries. A fundamental problem is that US agencies continue to hold differing definitions and area measurements for what constitutes the U.S. Arctic under ARPA. In fact, geographers at the State Department, NOAA, and the U.S. Coast Guard have recently expressed consternation over how imprecise mapping tools contribute to ongoing problems as basic as quantifying the size and extent of the U.S. Arctic. Making a standard shapefile of the US Arctic using the ARPA definition for the U.S. boundaries and extending to the Exclusive economic zone (EEZ) will help standardize the way US agencies represent the Arctic and calculate the various resources within it. Further, the existing Boundary maps provide no detail whatsoever about terrestrial features of Arctic Alaska, thus contributing to perpetual misunderstanding about the basic geography, scope and scale of Arctic matters. The fact that the Interagency Arctic Research Policy Committee is currently updating the National Arctic Science 5-year plan (2022-26) creates a high-profile opportunity for USGS to apply its geospatial mapping expertise to work with external partners to update and improve on previous tools for timely inclusion of new products in the next five-year plan. The maps and shapefiles will provide a widely used platform to deliver updated and integrated situational awareness and relevant modeling information for all stakeholders concerned about the features of a rapidly changing Arctic.

\section{Improved Decision-Support}

In total there will be at least five new map products as well as a newly standardized shapefile. The first map depicts the U.S. ARPA Boundary as it relates to Alaska and the Bering Sea. The second map depicts the Boundary from a circumpolar perspective. The third one depicts in poster size detail the Boundary as it relates to the terrestrial continental features of Arctic Alaska. The fourth one depicts in poster size detail the Boundary as it relates to the Aleutian chain terrestrial features of Arctic Alaska. A fifth map will represent the U.S. Arctic boundaries as a smaller subset of the ARPA Boundary map. A final sixth product will be an interactive web application that users can visit to interact with the maps in a selective and dynamic manner with automatically updated source files. A derivative product not owned or controlled by USGS will be a revised ARPA boundary shapefile with updated metadata information and guidance about how to reconstruct the boundary polygon for additional internal use by other agencies and stakeholders. 
The updated Boundary maps will improve both understanding and decision making concerning Arctic Alaska in many substantial ways. First, the new products will integrate multiple sets of observational data across disciplines and agencies into a pan-optic format. For example, the maps integrate refined Alaska state waters and shoreline vectors from NOAA, demographic information from US census data and State of Alaska Department of Natural Resources, sea ice coverage to show maximum extent from the National Snow and Ice Data Center, gridded international bathymetry data of the Arctic Ocean, terrestrial land cover from USGS, conservation areas by DOI land management agency, circumpolar July average 10 degree isotherm from the National Weather Service, and major circumpolar transit routes from the U.S. Coast Guard. Second, the new products will provide a basis from which stakeholders can readily grasp essential details necessary to understand fundamental Arctic scales, relationships and issues, and to easily monitor annual changes relevant to Arctic Alaska, such as sea ice extent, land cover, population clusters, and shifting climatological boundary lines. Third, the new products will juxtapose, in a single location, the various alternative scientific and political definitions of Arctic, including: the Arctic Circle, the circumpolar July average 10 degree isotherm, the boreal tree cover limit, and the U.S. Arctic Research Policy Act of 1984.

\section{State of the Science and Advancement Opportunities}

The proposed maps and web applications under construction will integrate relevant data collected by many different agencies. The maps themselves will not involve interpretive science products nor present the results of otherwise unpublished scientific findings. This effort does not advance the state of science so much as the pan-optic integration of relevant features for more convenient comprehension and use by decision-makers, as well as partners, stakeholders and the general public. Creation of the web application will provide a novel platform for delivery of additional layers of information that will undoubtedly grow over time as EarthMAP services gain momentum.

\section{Operational Implementation and Technical Considerations}

This Use Case was first conceived and launched in May of 2020 and has gained momentum and further definition through the summer and fall. Cooperation between the Alaska Regional Office and the National Geospatial Technical Operations Center of the Core Science Systems Mission Area has yielded a first round of draft map products by October. These products will be shared with external mapping specialists from partner agencies through a documented peer review process. It is anticipated that final products will be forthcoming and cleared for public dissemination in the fall of 2020 .

\section{Longevity and Sustainability}

Once created and finalized, these new map products and web applications will exist in perpetuity with a built-in mechanism for convenient updates. The Use Case will yield a very targeted and relatively high-profile success in delivery of new products and services that are directly responsive to expressed needs of state, federal, and international partners. These products will thereby also play a valuable role in promoting momentum for the EarthMAP vision and brand as USGS increasingly disseminates the concept externally.

Primary Contact: Dee Williams, dmwilliams@usgs.gov

\section{Enhanced Delivery of Integrated Ecosystem Model Output}

The Alaska Climate Adaptation Science Center (AKCASC), in collaboration with the University of Alaska-Fairbanks (UAF), will build on pre-existing partnerships to enhance the Alaska Integrated Ecosystem Model (IEM) by improving output of summary projections that simulate spatially explicit vegetation and wildfire responses to climate in sub-arctic and boreal landscapes. The project will increase USGS and partner capacity to access and use downscaled climate projections, develop impact models and geospatial information at regional and local scales, and enable better public understanding of output products.

\section{Demand and Relevance}

Assessments of climate-change effects on ecosystem processes and services in high-latitude regions are hindered by a lack of decision-support tools capable of forecasting and visualizing possible future landscapes under different scenarios. The increasing threat and occurrence of extensive wildfire in Alaska creates a compelling demand for improved weather observation and forecast (modeled) data. Direct beneficiaries of enhanced model output include the BLM Alaska Fire Service, State of Alaska Division of Forestry, NPS, U.S. Department of Agriculture (USDA) Forest Service, regional and local planning departments, and Alaska Native communities. This Use Case will move relevant climate information out of archival storage and into management decision spaces, and thereby help gage future fire hazard, reduce future wildfire risk, promote ecological resilience and wildlife habitat management, and demonstrate responsive action to Executive Order 13855 and Secretarial Order 3372. 


\section{Improved Decision-Support}

IEM output products are currently partially served on public databases, but largely in formats requiring considerable analytical expertise to access and process. Serving, sub-setting, and summarizing IEM/ALFRESCO (Alaska Frame-Based Ecosystem Code) output for the specific regions, processes, and species of interest to AKCASC and its collaborators would provide better opportunities for leveraged research on vegetation and wildlife responses. Specific tasks include: (1) summarizing the range and timing of vegetation changes across available future climate scenarios (RCPs) with an emphasis on imaging the most characteristic changes by geographical landscape or watershed; (2) identifying the landscapes likely to undergo the most rapid and severe changes as indicators of potential vulnerability and likely management challenges; (3) identifying the landscapes with habitat most likely to become rare and (or) more isolated; and (4) characterizing and comparing the landscape modeling versus climate scenario uncertainties across the replicates of model output to determine which scenario input is the most dominant driver of model output.

\section{State of the Science and Advancement Opportunities}

The IEM uses the model ALFRESCO to simulate spatially explicit vegetation and fire responses to climate. Future climate scenarios downscaled from global climate models (Meteorological Research Institute CGCM3 and National Center for Atmospheric Research CCSM4) run under different greenhouse gas scenarios have already been used as input to ALFRESCO to develop a large library of replicated future landscape simulations (200 replicates per scenario account for stochasticity in the vegetation model). Summary variables comparing historical (1900-99) and current-to-future (2000-99) landscape characteristics, such as the change in number of fires per century or change in number of vegetation transitions, illustrate the relative changes expected across different parts of Alaska. However, the detailed projections associated with these simulations also allow exploration of other problems, such as habitat changes associated with different vegetation types important to wildlife (for example, changes from spruce to deciduous forest, increases in shrub-tundra, or changes in tree line). To date, these datasets are largely unexplored.

\section{Operational Implementation and Technical Considerations}

The AKCASC will provide UAF with $\$ 450,000$ in continuing support of IEM, while the AK Regional Office (with contributions from the Southeast, Midcontinent, and Rocky Mountain Regions) will provide $\$ 300,000$ to support a work order modification to launch the Use Case. The summary output maps and narratives will benefit from a separately funded work order to develop an improved web interface tool for more seamless interactive geographic display. Data currently exists in NetCDF format at data centers in Fairbanks. In exploration of additional national utility, the Use Case will also actively test a potential university-USGS-Tableau interface built on an Amazon Web Services platform.

\section{Longevity and Sustainability}

Upon completion of the work order modification, a targeted stakeholder workshop is intended to occur in 2021 to evaluate next steps. The workshop will assess the utility of new model summary output products and evaluate prospects to sustain additional effort over the lifetime of the management-action framework, including data storage and delivery obstacles.

Primary Contact: Steve Gray, sgray@usgs.gov

\section{Mid-Continent Region}

\section{EarthMAP as a Strategic, Systematic Interface Between U.S. Geological Survey and the National Park Service Resource Stewardship Strategies}

EarthMAP is intended to work at the interface between USGS science and managers' information needs; the National Park Service (NPS) creates Resource Stewardship Strategies (RSS) to define the desired conditions of each park's natural and cultural resources and could be seen as the NPS side of an EarthMAP-manager interface. We propose an EarthMAP-national parks' Use Case to understand how the USGS might work with NPS to provide management relevant information pre- and post-implementation of actions undertaken to meet RSS goals.

\section{Demand and Relevance}

Decision(s) this EarthMAP Use Case will informHow national parks implement their Resource Stewardship Strategies (RSS). Gauge the value of RSS-relevant information to park natural resource managers, devise plans for USGS delivery of valuable information for RSS implementation, and provide systems of analysis to judge efficacy of RSS implementation.

Relevant decision-makers, stakeholders, and intended information recipients; their interests in and need for improved science information-NPS natural resource managers. The RSS sets resource management goals but implementation is limited by the complexities of dealing with interacting threats, poor understanding of vulnerabilities and species adaptive capacity, and inconsistent monitoring of management effects. 
Integrated science and information synthesis necessary to address the problem in context of EarthMAP and the decision(s) to be made. Use Indiana Dunes National Park (INDU) as a prototype for RSS-relevant information delivery by USGS. INDU has the range of landscapes for which RSS-relevant information is needed - from the waters of Lake Michigan to coastal dunal ecosystems to coastal and inland wetlands to rivers, forests, savannas, and prairies. There is a need to synthesize information for a similar range of landscapes across the national park system, providing continuing information on rates of change of a variety of geomorphological (for example, coastal erosion at INDU), hydrological (for example, lake level effects on ecosystem processes at INDU), land use change (for example, Land Change Monitoring, Assessment, and Projection [LCMAP]), and visitor impact trends (for example, remote sensing of social trail development) as well as ecological characteristics such as diversity, resilience, productivity, vulnerability, and connectivity. This information would need to be delivered in at least yearly timesteps. Although implementation of an RSS has park specific aspects, the physical and ecological characteristics listed are representative of a suite of management relevant characteristics that could be honed through Use Cases and made relevant to all parks. Through this Use Case, data gathering and maintenance burden on the parks for RSS relevant information should be minimized if this system of information provision is to be expandable to other parks for the long term.

The RSS is a primary management document for a national park providing "an objective basis for assessing the condition of natural and cultural resources relative to the "desired conditions" established in the park General Management Plan, and (2) to document science- and scholarship-based comprehensive strategies to achieve and maintain these desired conditions." The process linking USGS capabilities in risk management to RSS needs would emphasize multiple EarthMAP priorities especially "Modeling and forecasting species distribution, abundance, and activity, including drivers and impacts, across multiple spatial and temporal scales" since resource managers often understand their jobs in terms of the species they have responsibility for (FY2021 Annual Program Guidance Ecosystems Mission Area, p 7).

The proposed process, although initially focused on providing a prototype national park more automated delivery of information on status of species and physical processes through time, should improve in resolution as model quality improves and should be applicable across parks if the metrics of landscape quality are honed to be of significant management relevance and generality regardless of the specific circumstances of a given park. Careful selection of metrics can increase the likelihood that they will all be useful for decision making or at least all parks will find useful metrics served from among the metrics presented.

\section{Improved Decision-Support}

We propose a system of data collection, analysis and prediction that will be generally relevant to the RSS process because it will provide feedback on primary features of importance for the mission of conservation of desired landscape and species conditions and restoration of those conditions when they are not presently met. For example, we anticipate that managers will generally be able to act on RSS goals better if they understand the status of landscape characteristics such as species diversity, resistance of biotic communities and physical attributes to change, productivity, vulnerability, and connectivity. However, that is an assumption to be investigated. Therefore, the first USGS skill set to bring to this protype process of melding RSS and EarthMAP is decision support expertise. DOI decision support experts would have an upfront role investigating how NPS resource managers use information - what is valued information in their decision-making process? Can we create a consistent universe of updatable high value characteristics of NPS managed landscapes to be delivered by EarthMAP?

The Indiana Dunes landscape is often considered to be the birthplace of the modern science of ecology_-"Ecological pioneers Henry Chandler Cowles and Victor Shelford discovered a time machine here" since they noted that the arrangement of habitats across the landscape was consistent with habitat succession through time (Jackson, 2011). It may well be the most degraded of the 62 current national parks, in part due to being embedded in one of the most heavily industrialized and populated landscapes in the U.S. Today in the park, USGS explores species composition of offshore communities in the park's Lake Michigan waters with autonomous underwater vehicles and research vessel-based surveys, remotely monitors animal calls, monitors species, native and invasive, in waterways using eDNA, and is publishing a detailed flora of one of the smallest (6000 ha) yet biodiverse ( $\sim 1200$ native plant species) national parks. The USGS has worked with INDU's resource management staff in creation of their RSS and with the Great Lakes Research and Education Center that coordinates research across the Great Lakes National Parks and is a key participant in bringing relevant science to the parks.

This prototype RSS-EarthMAP Use Case has the possibility of forging an important proof of concept of the utility of EarthMAP for guiding more effective restoration and conservation decisions in national parks. National parks can broadly be classified as "conservation parks" and "restoration parks," that is, parks whose management efforts are more aimed at maintaining a status quo by building in resistance to change and parks that are managed with the intent of changing the landscape. As a highly degraded yet biodiverse national park Indiana Dunes could best serve as a prototype of the interaction between the restoration goals emphasized in the Indiana Dunes RSS and the guiding information provided by EarthMAP. 


\section{Science Capabilities and Advancement Opportunities}

Indiana Dunes is an extensively studied and monitored park with a long history of ecological scientific inquiry and a robust commitment to fostering scientific inquiry that was foundational to the creation of the park's RSS. Nonetheless, implementation of the RSS, and the validity of the RSS goals, are still only weakly supported by ongoing understanding of status and rates of change of the underlying biological communities and physical environment. There are many USGS datasets that could be consistently useful for updating the managers' toobox for Indiana Dunes and other national parks. For instance, as NLCD becomes more frequently updated and as USGS products such as LCMAP become more adept at documenting land use change, we can regularly update the status of connectivity across a park and predict future changes more accurately in the park and in the greater ecosystem surrounding the park. We can use emerging models of microclimatic prediction, along with climate projections as an entrée into developing and serving metrics of landscape resilience, such as ability of landscapes to retain species as microclimatic patterns change. Metrics of this sort can be combined to give the RSS implementor access to a compendium of landscape characterization, for example, how connected and resilient is a particular property.

However, perhaps the most important USGS dataset that could be stood up in an improved way to be useful to RSS implementation is the USGS GAP models of species distribution. There are thousands of terrestrial and aquatic models available through this program. In their current state, many could be useful for the RSS implementation process by, for example, predicting likely patterns of diversity across parks. However, these models need to be supplanted with better, perhaps more biophysically based models that can provide more realistic estimates of distribution and likely species abundance and distribution change with environmental change. Currently these are mainly statistical models that compare observations of a species with basic environmental datasets, such as NLCD, to produce correlative models of habitat associations versus providing an understanding of the biophysical niche of the species and how variations and changes in the environmental underpinnings of that biophysical niche might affect distribution. Trying to use the current GAP models to estimate biotic metrics of utility to the RSS, such as biodiversity distributional patterns, will likely highlight the weaknesses of the GAP models but underscore the likely utility of better species distribution models for resource management use. The USGS is a world leader in species distribution modeling and national parks are relatively well documented repositories of species distribution observations and locales where automated species monitoring may relatively quickly become standardized and implemented. As such they should be good proving grounds for general ways to improve species distribution models in a manner that would help managers predict the effects of their management actions more accurately. In this RSS framework we could imagine a system of species distribution model improvement and management scenario automation coming together to guide how park resource managers game out their management options.

\section{Operational Implementation and Technical Considerations}

One reason to suggest Indiana Dunes National Park as a prototype for interfacing RSS and EarthMAP is that the USGS has produced park-specific preliminary manifestations of some of the likely RSS-relevant EarthMAP outputs - maps of INDU wildlife corridors USGS are an example. However, while USGS has a significant history of responding to NPS information needs at this park, it does not have the history, or current capacity, of delivering a system of analysis and prediction to the park that would provide readily updateable, management relevant metrics that the NPS would receive with minimal effort on their part. The process of vetting the value of information in a systematic way, creating or upgrading predictive models of species distribution and environmental change, and serving this information in a manner that doesn't require unattainable amounts of continued work from park resource management staff will probably move approximately at the speed of funding but that's the challenge of the EarthMAP program. Even if we focus the management relevant information delivery to ecological information mainly related to species distribution, we are proposing this as a testbed for significantly improving management relevant species distribution modeling and that part alone will require new resources. There will be a premium on managing dataflows feeding updated models and new metrics in an automated manner that can respond flexibly to NPS management scenarios, that is, systems that provide answers when managers ask "what if." 


\section{Longevity and Sustainability}

RSS-EarthMAP will sustain its relevance if it becomes foundational to the management of parks and has components that will examine how managers use information. If successful, the system would be updateable and would have to be maintained at a national level. The system would be applicable across multiple park RSS so would become national in scope. Because the RSS are not completed nationally, it is likely that the two programs would influence each other's growth so that the success of EarthMAP in providing actionable intelligence for the RSS implementation, and documentation of the effects of RSS implementation, will likely affect how the RSS are designed in subsequent parks. The RSS and EarthMAP products would interact in an adaptive management framework. For instance, a possible EarthMAP product might include resilience measures, such as how remote sensed primary productivity is affected by variations in climate and how management of different areas of a park affect the degree to which productivity changes as weather varies. As the parks learn more about how their management does, or does not, impart productivity resilience, RSS may be modified or created to consider how successful management is in producing landscapes that resist changes in productivity.

Primary Contact: Ralph Grundel, rgrundel@usgs.gov

\section{U.S. Geological Survey Integrated Ecohydrology Science: A New Path to Advance Water Science, Ecosystem Science, and Earth-System Prediction}

Water-related stressors (for example, drought, floods, contamination) pose serious challenges to the resilience of human communities and ecosystem services, and there is substantial capacity for USGS to support resource management decision making at the nexus of water, ecosystems, and society across the Nation. We present an innovative ecohydrological framework for USGS integrated water and ecosystem science that addresses the current and future vulnerability and volatility of water resources, the ecosystems they support, and the services these ecosystems provide to deliver actionable information to decision-makers and stakeholders.

\section{Demand and Relevance}

Scientific and management communities increasingly recognize the vital role of hydrologic and water quality variability as fundamental drivers of ecosystems and the numerous services they provide to society. Of particular concern are the Nation's rapidly changing and declining water supplies, the emergence of extreme events under warmer conditions, and alteration of land use and landcover as major stressors to human and ecological systems. Hazards from water quantity (both low and high) represent the second (drought) and fourth (flood) most costly weather and climate disasters in the United States. However, understanding the processes influencing the availability, movement, and quality of water across landscapes is currently hindered by the location, extent, and scale of current hydrologic monitoring. Additionally, there is a significant misalignment of hydrological, physical, chemical, and biological data across space and time to understand and predict how changes in the quantity and quality of water affect important ecosystem services and how these changes might be mitigated. Consequently, there is an urgent need to advance USGS integrated water and ecosystem science to inform decision making across the United States.

Ecohydrology, the interdisciplinary scientific field focused on interactions and associated feedbacks in both space and time between water and ecological systems, presents a unique opportunity to advance EarthMAP by developing, integrating, and applying methods and tools for water observation and prediction while conducting underlying interdisciplinary ecohydrological science to quantify ecosystem services and address stakeholder needs. This effort requires integration of USGS expertise and capabilities and will allow USGS to bring its full science capacity to bear on complex systems where biological, ecological, and water-related factors interact, affecting species, habitats, and ecosystem services important to society. This effort will directly benefit key stakeholders concerned with water-related issues from all regions of the United States, including Federal regulatory and management agencies, such as the FWS, BLM, Bureau of Reclamation (BoR), NPS, USFS, USDA, U.S. Environmental Protection Agency (EPA), USACE, National Oceanic and Atmospheric Administration etc.; State agencies, such as departments of natural resources, fish and wildlife, public health, and water resources; cities and counties; hydropower companies; farmers and landowners; and Tribes. 


\section{Improved Decision-Support}

This EarthMAP project seeks to provide managers, decision makers, and the public with actionable information at multiple relevant spatial and temporal scales about surface-water availability, stream-network connectivity, groundwater level fluctuations, water quality, and ecosystem effects. By integrating these types of data, USGS can provide actionable information to stakeholders so they make more informed decisions regarding water resources and ecosystems that impact issues such as fishery condition, irrigation potential, land productivity, vegetation changes, conservation practices, and recreational resources. Irrespective of the basin and its overarching focus, development of specific water and ecosystem-service modules will be informed by diverse stakeholder engagement (for example, interagency team) and co-production of stakeholder-relevant data products. Thus, we envision a dynamic system that provides managers with timely water quantity, quality, connectivity, and extent information, and associated cascading impacts on vegetation patterns, ecosystem services, recreational use, socioeconomic stability, and others. Specifically, the goal of our project is to produce daily current, short-, mid-, and long-range predictions of streamflow discharge, probabilistic risk of hazard (for example, flood or drought), stream-network connectivity, wetland extent, and relative concentrations of ecologically relevant contaminants, presented at multiple relevant ecohydrologic scales (for example, reach, basin, region) for all locations in the Midcontinent Region. Further, biological data (for example, growth, survival, distribution, abundance, diversity, eDNA data for aquatic and terrestrial species/communities) that align with the probabilistic outputs from our model will be collected and (or) aggregated and served through our visualization platform. These combined products will facilitate integrated analyses to better understand the ecohydrologic connections and feedbacks in the system, ultimately providing more informed resource conservation and management.

\section{Science Capabilities and Advancement Opportunities}

This effort represents a great opportunity to integrate USGS expertise and capabilities within and across mission areas, including Water Resources, Energy and Minerals, Core Science Systems, and Natural Hazards (fig. 5.3). USGS has well-established, but separate, research themes focused on water resources including the quantity and quality of water, long-term trends in water availability, and improving abilities to forecast water availability; contaminant sources and prevalence, fate/transport, exposure, aquatic toxicity; and the biology and habitat needs of aquatic-dependent animals and plants.

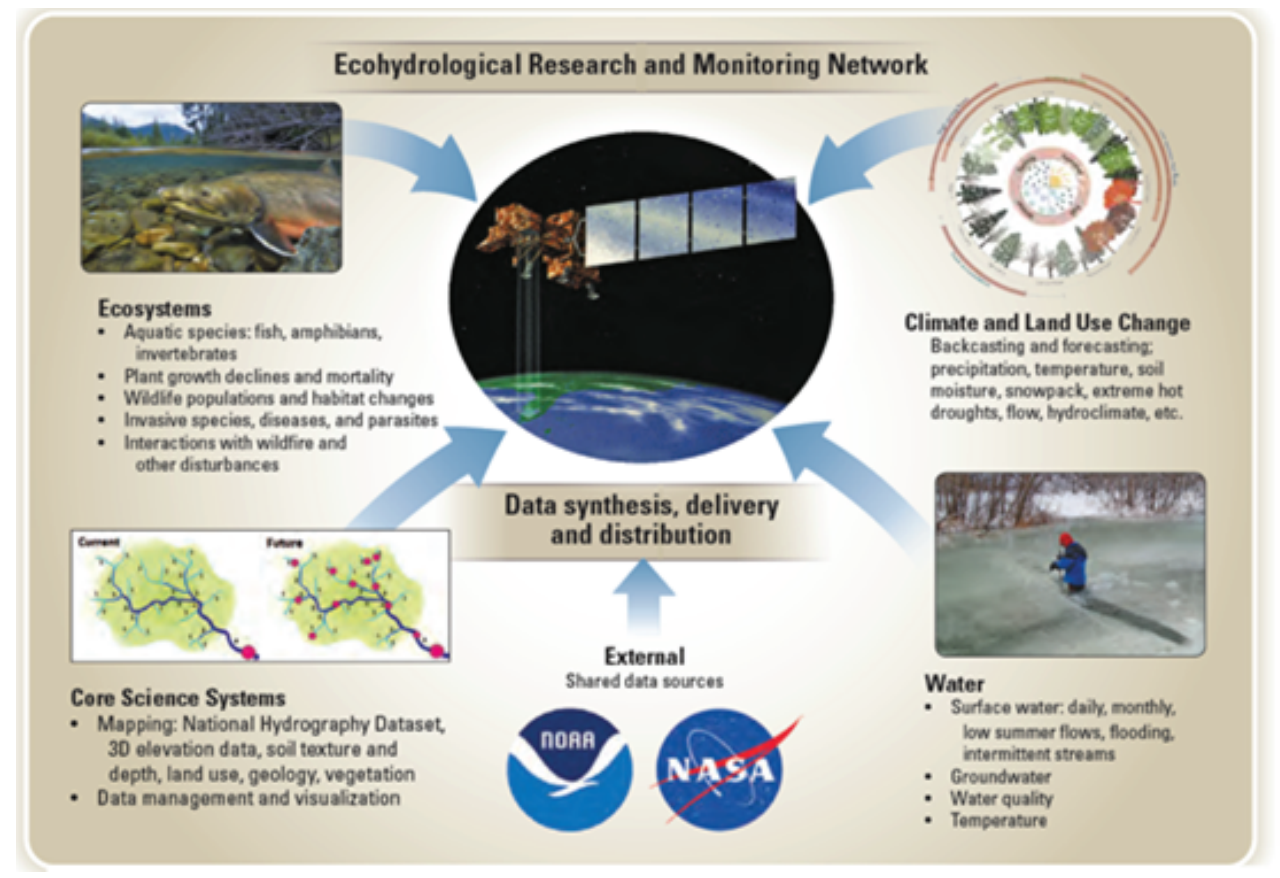

Figure 5.3. Conceptual depiction of the U.S. Geological Survey (USGS) programs and elements that will be integrated into USGS Integrated Ecohydrology Science. 
This new path for USGS to advance coordinated and integrated multidisciplinary science will also contribute to broader Bureau-level priorities, including other applications of EarthMAP and the USGS 21st Century Science Strategy (U.S. Geological Survey, 2021), both of which focus on data integration and modeling, and the production and delivery of actionable information. The USGS possesses considerable resources and capabilities in regard to geology/vadose/soil mapping, water monitoring, water flow and quality modeling, ecosystem-service modeling, land-use/landcover mapping, vegetation sampling and mapping, and animal population dynamics and habitat needs that will make this project successful. For example, by leveraging USGS strengths (for example, Integrated Water Availability Assessments [IWAAs], Next Generation Water Observing System [NGWOS], USGS Ecological Drought Program, Risk Community of Practice [CoP], Community for Data Integration [CDI], Science Analytics and Synthesis, USGS Climate Adaptation Science Centers [CASCs], Great lakes Restoration Initiative [GLRI]) this effort could benefit the EarthMAP pilot project focused on drought in the Colorado River Basin or could support the emerging USGS Initiative focused on post-fire hazards, risk assessments, and decision making in western US watersheds. It will also create opportunities to strengthen Congressional support across administrations, and to enhance and forge collaborative relationships across the Bureau and with other agencies. This multi-Mission pilot project could be broadened to consider the full spectrum of ecohydrological issues relevant to stakeholders, from floods to droughts, and from stream-bottoms to terrestrial systems.

\section{Operational Implementation and Technical Considerations}

USGS Ecohydrological Science provides an adaptive science framework to observe, synthesize, model, forecast, and deliver decision-support guidance to address current and future management needs, while monitoring, revising, and improving tools and technologies to advance water and ecosystem science in a changing world. The first step of implementing this approach is to assess existing key data and platforms, including surface water, groundwater, water quality, water temperature, water use, soil moisture, snowpack, ecology and the supporting national hydro-geospatial framework. For example, modeling products for water quantity (PROSPER model, Precipitation-Runoff Modeling System, Monthly Water Balance Model, Soil-Water-Balance Model, Operational Simplified Surface Energy Balance, WRF-hydro) and water quality (SPARROW, RHyME2 [Regional Hydrologic Modeling for Environmental Evaluation], SWAT [Soil and Water Assessment Tool]) will be scoured from the newly developed USGS Model Catalog, compiled and synchronized. Second, strengthening and broadening data observation networks and predictions are needed to spatial and temporal coverage of space-based, airborne, aquatic, and terrestrial data observation networks that collect information at multiple spatial scales. Some examples of existing platforms include the USGS National Water Information System, the National Water Quality Portal, eDNA databases, dynamic citizen science efforts (for example, StreamTracker, FLOwPER [FLOwPERmanence]), various State- and watershed-specific databases, and others. A state-of-the-art data-exchange, storage, and delivery infrastructure will be important to support these new robust networks and deliver near- or real-time data. This project will coordinate with efforts like the Water Resources Mission Area NWIS [National Water Information System) Modernization project to ensure we are at the forefront of delivery mechanisms. These models and data will be aggregated by appropriate spatial and temporal scales, and subsequently linked to the National Hydrography Dataset (NHD). Next, coordination of these strengthened data observations will enable USGS scientists and partners to synthesize datasets and models to improve understanding of drivers, responses, and interactions of water-related issues facing ecosystems and society. This information will improve our ability to forecast vulnerable and resilient systems. Finally, these steps in the process to strengthen, synthesize, and predict, will require increased coordination across USGS Mission Areas and partners, but will ultimately be essential to build and deliver decision-support to address important decision making and stakeholder needs. This new integrated and predictive science will help Federal, State, Tribal, and local resource managers with improved planning, strategic implementation and mitigation strategies.

The final output from this project will be an interactive Web interface that provides users with daily current, short-, mid-, and long-range predictions of streamflow discharge, probabilistic risk of hazard (for example, flood or drought), stream-network connectivity, wetland extent, and relative concentrations of ecologically relevant contaminants, presented at multiple relevant ecohydrologic scales (for example, reach, basin, region) for all locations in the Midcontinent Region. Additionally, biological data (for example, aquatic productivity, threatened and species of special concern, invasive species, food web dynamics, eDNA) that align with the probabilistic outputs from our model will be collected and (or) aggregated and served through our visualization platform. Hydrologic outputs could also be used as inputs to risk models to predict the probability of aquatic invasive species (for example, Dreissenid mussels, Carp, Eurasian watermilfoil, smallmouth bass, invasive trout and char, northern pike etc.) invasion, establishment, and spread. 
In the first phase of this work, the integrated ecohydrology framework will be applied to three pilot basins in the Midcontinent Region, representing different ecohydrologic contexts and issues (Upper Missouri River basin, Mississippi River basin, and The Great Lakes). These basins will be selected based on the availability of data, the diversity and representativity of ecohydrologic conditions, socioeconomic importance, and partner engagement.

Synopsis of potential ecoregion foci, including ecohydrologic issue addressed:

- Missouri River basin - drought, stream connectivity, water quantity and quality, invasive species impacts

- Mississippi River basin-flooding, water quality

- Great Lakes basin-water quality, lake level changes, invasive species impacts

\section{Longevity and Sustainability}

Water-related stress has been studied by the USGS for over a century and humans have benefited from associated ecosystem services for millennia. The relevance of this EarthMAP application will be permanent as long as humans interact and use the landscape since foresters, agriculturalists, land managers and the public will need to know about, mitigate, and adapt to impacts to water supply/quality and ecosystem services that affect their work, livelihood and recreation. The methods and results of this Use Case will be applicable to any watershed or landscape. Further, the integrated data, models, and outputs will be archived in the cloud and independent of the lifecycle associated with locally established servers. Miminal upkeep will be required annually to ensure the operational success of the outputs. Effectiveness of the effort will be evaluated and quantified by the extent of stakeholder engagement, public and manager use of the tools, the effectiveness of tools to accurately portray conditions, and the level of synthesis/integration among earth systems.

Primary Contact: Clint Muhlfeld,cmuhlfeld@usgs.gov

\section{Harmful Algal Bloom Monitoring and Prediction (HABMAP)}

Critical information is needed for decision makers regarding harmful algal bloom (HAB) occurrence and toxicity. USGS has numerous existing capabilities related to toxic algal blooms that can be used for HAB prediction, including site-specific streamflow and water-quality monitoring,laboratory analyses and experiments, land use information,satellite imagery and associated ground-truthing, predictive modeling for toxins, as well as promising emerging technologies.

\section{Demand and Relevance}

There is a need to integrate the data being collected to better understand triggers for bloom development, persistence, toxicity, and to improve predictive capabilities. Cyanobacteria harmful algal blooms (cyanoHmonABs) and nuisance algal overgrowth cause a variety of environmental health issues that can also have significant socioeconomic consequences in rivers, lakes, wetlands, estuaries, and oceans. We have limited ability to predict variation in cyanotoxin concentrations at large spatial or temporal scales and limited ability to predict HAB-related toxins at all spatial and temporal scales. Land managers and managers of waters for municipal, industrial, and recreational purposes could use predictions of the location and timing of high toxicity HABs and nuisance washup events to mitigate or minimize their environmental health and economic impacts (for example, by switching water sources, posting warnings, etc.).

USGS collects and analyzes hydrological, chemical, environmental, biological, geological, land-use and many other types of data from multiple locations, at a wide range of spatial and temporal scales. Each of these types of data have an impact on the development and character of HABs. The EarthMAP concept can be used to connect this information together in the context of HABs (HABMAP) to not only help USGS provide answers to one of societies critical problems but also to develop the procedures and mechanism needed to apply EarthMAP to other integrated science questions.

Managers and users of waters for municipal, industrial, and recreational purposes could use predictions of the location and timing of high toxicity and nuisance washup events to minimize the environmental health and economic impacts of harmful algal blooms (for example, by adjusting or switching water sources, triggering sample collection, posting warnings, etc.). Improvements in predictability and minimization of harmful effects of algal blooms have been identified as high priority issues in USGS Ecosystems and Water Resources Mission Areas and have been highlighted as part of the Great Lakes Restoration Initiative (GLRI). 
Meeting this demand could integrate information to understand, manage, and mitigate factors that lead to HABs with the ultimate goal of building predictive models for a wide variety of ecosystems across North America. The information could be used to develop a well-documented and approved methodology for incorporating available predicted effects of future climate-change with current USGS site-specific streamflow data and information (current hydrographs, flow-duration hydrographs, streamflow statistics, etc.) into a nation-wide decision support tool for planners to use. The proposed HABMAP could incorporate indicators of uncertainty in results and use automated data processing to continuously update available climate scenarios into streamflow information and apply machine learning to develop flow-related predictions of loads and concentrations for selected constituents. The integration of data could also be used to estimate cyanobacteria cell abundance in larger lakes and reservoirs of the United States to assist federal agencies, tribes, states, municipalities, and drinking water utilities with surface water management decision support. Managers and users of waters for municipal, industrial, and recreational purposes could use predictions of the location and timing of high toxicity events to minimize the environmental health and economic impacts of harmful algal blooms (for example, by switching water sources, posting warnings, etc.).

Potential collaborators include USGS Mission Areas Ecosystems, Water Resources, Natural Hazards; Public health officials, municipal planners, agronomists, and the public; Federal Agencies (USACE, FEMA [Federal Emergency Management Agency], EPA, NASA [National Aeronautics and Space Administration], FWS, DOD [Department of Defense], DOT [U.S. Department of Transportation], HUD [U.S. Housing and Urban Development], etc.); State Agencies (Water supply, water quality [DEQ; Department of Environmental Quality], DNR [Department of Natural Resources], economic development, etc.); Local and Tribal Agencies (Water Departments, Waste-water/stormwater planners, Public Works, etc.); University researchers, Engineering companies, Environmental groups, etc.

\section{Improved Decision-Support}

Federal, State and local health and environmental agencies are highly interested in using real-time data and information to improve $\mathrm{HAB}$ identification and reduce the cost of identifying HABs, sampling, determining cell abundance/ biovolume, and estimating toxin concentrations to provide meaningful and actionable information related to protection of health and the environment.

Our partners know they need a decision support tool for information related to HABs. They also provide great help with respect to site-specific information, water-quality data, land use data, water use as well as other pertinent information needed to improve our ability to predict HABs. This kind of decision support tool will provide an expanded opportunity to involve our stakeholders in USGS science and this has already occurred in studies where we are already developing predictive models and tools.

The expected impact of this decision support tool is use by managers and users of waters for municipal, industrial, and recreational purposes throughout the nation to better predict the location and timing of high toxicity events to minimize the environmental health and economic impacts of harmful algal blooms.

We will build on a long history of local and regional research including in situ data collection, remote sensing and imagery, data analysis and evaluation, and statistical and process models to develop a field to rivers, lakes, wetlands, estuaries, and oceans understanding of the factors controlling HABs formation.

\section{Science Capabilities and Advancement Opportunities}

USGS will use existing or new sites to collect discrete and instantaneously measured water-quality data, field fluorescence and lab values of algal pigment data, phytoplankton community composition and abundance data, field-based hyperspectral imaging, and other relevant data such as wind speed and direction and photosynthetically active radiation (PAR). Surrogate (proxy) models will be developed to relate continuously measured data to discretely sampled data and once verified, data from days and time of scheduled satellite overflights will be used to verify and calibrate the relation between satellite data and site-based data using machine learning and automated data processing. At recreational and drinking-water treatment plant intake sites where data are available, statistical models will be developed to estimate toxin concentrations.

Existing infrastructure in USGS could be integrated for accurate bloom predictions, including real-time measurements of nutrient inputs and eDNA of harmful algae using the next-generation streamgage network, applied in both stream and lake settings, and geospatial mapping of bloom biomass through the Cyanobacteria Assessment Network (CYAN) project. Additional capacity, for example, in remote sensing of benthic algae, hyperspectral imaging of algal types, Autonomous Underwater Vehicle (AUV) mapping, and hydrodynamic models for nutrient and algal transport, could be developed within USGS or through collaborations to complete the analysis and prediction capabilities. We are using molecular tools to measure genetic potential, toxin production intermediaries and toxin composition in a range of laboratory and wild cyanobacterial populations. Molecular data also is used in statistical models to provide advanced warning of a toxic HAB. 
Partnerships could form between USGS Ecosystems Mission Area, Environmental Health Program, Water Resources Mission Area, Natural Hazards Mission Area, Great Lakes Restoration Initiative (GLRI); Public health officials, municipal planners, agronomists, and the public; Federal Agencies (USACE, FEMA, EPA, NASA, FWS, DOD, DOT, HUD, etc.); State Agencies (Water supply, water quality [DEQ], DNR, economic development, etc.); Local and Tribal Agencies (Water Departments, Waste-water/stormwater planners, Public Works, etc.); University researchers, engineering companies, environmental groups, and others.

\section{Operational Implementation and Technical Considerations}

USGS has broad and expanding capabilities across the Region and Science Centers to facilitate the Use Case for HABMAP. With established programs in nutrient loading, streamflow, genomics, and survey assessments across the fields of hydrology, limnology, hydrodynamics, molecular biology, remote sensing, data analysis and modeling. Ongoing research activities include an interagency effort (EPA, NASA, NOAA, USGS) and targeted efforts (for example, GLRI) to develop the Cyanobacteria Assessment Network (CyAN) that utilizes remote sensing to estimate cyanobacteria cell abundance in larger lakes and reservoirs. Another Center is using sophisticated molecular tools to measure genetic potential, toxin production intermediaries, and toxin composition in cyanobacterial composition. Ongoing basinwide research exploring the drivers of nuisance algae (Cladophora) can also be leveraged to identify simultaneously drivers of multiple toxic and nuisance algal blooms, along with partnering agencies and academia (Michigan Tech, Michigan State Univ.). The Great Lakes NowCast, which currently provides estimates of $E$. coli concentrations at recreational sites using predictive models, could be expanded to include toxin estimates at recreational drinking-water intake sites throughout the region.

\section{Longevity and Sustainability}

Without a better understanding of the triggers for bloom development, persistence, and toxicity at a large scale, and with potential future impacts of climate-change, the HABMAP Use Case has long-term relevance for the nation as HAB-related issues continue to impact users of waters for municipal, industrial, and recreational purposes.

Data from our large monitoring network will be used to evaluate and improve the effectiveness of decision support tool prediction and modelling efforts, and define metrics of performance and success.
Leveraging of research programs will assist in long-term viability of the HABMAP Use Case. USGS has an established reputation examining bloom drivers and predictive models and has established a large network of partners and stakeholders. Partners have an interest in maintaining a scale approach to HABs that will inform their management of local and regional actions that can be taken to improve algal blooms. This scaling approach could incorporate local streamgages and directed sample collection. The approach taken in HABMAP will benefit research in Great Lakes and other waterbodies that have stressors driven by similar conditions: toxic and nuisance algae, agricultural runoff, municipal nutrient loading, urban ecosystems, drinking water, and shoreline recreational activities.

Primary Contact: Victoria Christensen, vglenn@usgs.gov

\section{Legacy Sources of Nutrients and Implications for Management Strategies}

Discussions of how to limit nutrient input to downstream receiving water bodies frequently focus on ongoing management upstream in the watershed; however, resource managers also need to know where, how much, and the form and mobility of nutrient pools in soil, groundwater, and surface water that provide baseline contributions and that supplement the ongoing inputs. This Use Case will provide decision makers the information needed to address nutrient management by quantifying the legacy sources that have accumulated on the scale of years to decades, and that have distinct temporal and geospatial signatures.

\section{Demand and Relevance}

"I'm using these costly management practices to keep the nutrients out of my water, and you're telling me that my water quality hasn't improved?!" This or similar questions are frequently voiced by stakeholders frustrated that investment in nutrient management, as best management practices, has shown minimal effect. We hypothesize that part of why significant change hasn't been observed is that legacy nutrient sources, which reflect more than a century of agricultural production and purposeful application of fertilizer and manure, provide a steady fux of both nitrogen $(N)$ and phosphorus $(P)$ which has accumulated in groundwater, upland soil, and sediment stored in streams and water bodies. Communicating the longevity and magnitude of legacy nutrients to stakeholders is key to preventing a loss of confidence in their ability to manage and improve their water quality." 
Just as predicting streamflow requires an understanding of antecedant conditions combined with recent events, so does the prediction of nutrient concentrations and loads. Nutrient concentrations are linked with recent land management, thus the interest in edge-of-field monitoring, which directly links field management and fluctuations in nutrient and sediment concentrations. In streams, seasonal fluctuations are also observable; however, new additions of nutrients are supplemented by contributions during baseflow and stream-energy driven erosion and redistribution of in-stream sediment. Similarly, nutrients stored in bottom sediments of water bodies can be released when water temperatures increase or bottom waters become depleted in oxygen - common occurrences in summer and early fall. Therefore, legacy sources can directly drive summer and early fall concentrations that foster hypoxia and harmful algal blooms (HABs).

\section{Current Questions Asked by Both Researchers and Resource Managers}

How will nitrate concentrations in groundwater decrease? Will this be spatially consistent? A function of local aquifer properties? Will mixing outpace exfiltration as baseflow?

How will orthophosphate (ortho-P) and sediment-bound $\mathrm{P}$ (sed-P) interact? Recent research shows that suspended sediment is enriched in sed-P relative to upland sources; however, it is unknown if this is true in streams of all sizes and how this varies as a function of ortho-P concentrations.

How much of the legacy P pool is bioavailable, specifically the sed-P component which likely varies in grain size, age, and submersion depth along individual stream networks?

How much does stream condition (temperature, discharge, and depth) affect bioavailability of legacy $\mathrm{P}$ and how will this change as seasonality of precipitation and nighttime temperatures change as a function of projected climate?

How important is nutrient release from sediment stored in stream channels and lakes to the overall nutrient budget of the downstream receiving waterbodies?

How does a better understanding of legacy nutrients change our anticipated changes in water quality based on current management actions taken throughout the watershed?

\section{Improved Decision-Support}

Discernable improvement in water quality of impaired waters with large sources of legacy nutrients requires a toolbox of monitoring and management strategies that address both current and legacy sources. It is also important to know which tool in that toolbox is most appropriate for meeting management goals for a given source (fig. 5.4). Legacy pools of soluble nutrients (principally nitrate and phosphate) in groundwater can strongly influence drinking water supplies and baseline conditions and will inherently cause a lag in the observed water-quality benefits of nutrient reductions to upland surfaces. The legacy pools of sediment-bound nutrients (principally phosphorus) in upland soil, streambanks, streambeds, lake sediments, and overbank sediment act as a source and sink for nutrients over different time scales, seasons, and flow conditions. Management techniques may range from on-field conservation practices that reduce nutrient transport and nutrient accumulation in soils to riparian buffers and stream restoration measures that trap nutrients and increase microbially-mediated nitrogen removal to inlake management actions. However, real-time, geospatially-integrated quantification and understanding of these different sources are needed to inform decisions for the prioritization of management options and to provide an understanding of when to expect improvement.

A better understanding of the importance of legacy nutrients is needed by groups such as the Gulf of Mexico Hypoxia Task Force, the Mississippi River Cities and Towns Initiative, parties to the Great Lakes Water Quality Agreement, the Groundwater Foundation, and many other groups that are working to improve water quality across the country.

\section{Science Capabilities and Advancement Opportunities}

USGS has developed large-scale surface-water models (for example, SPARROW) and regional groundwater models (for example, Modflow) to describe generalized nutrient transport over large areas, whereas small-scale studies have generally focused on specific transport mechanisms and differentiation of new sources of nutrients and legacy sources (for example, SWAT, sediment fingerprinting, empirical to hydrodynamic-ecological models). However, we currently lack watershed models and communication strategies that couple these approaches and integrate our understanding of nutrient transport processes, especially with respect to legacy pools of nutrients, to enable large-scale predictions in water quality that drive HABs, drinking water restrictions, and hypoxia. Only with a better understanding of all of the sources of the problem - nutrient loading from both current and legacy sources - can we expect to describe variability and forecast change in HABs in the Great Lakes and other water supplies as well as hypoxia in lakes, estuaries, and the Gulf of Mexico.

Current science methods can be advanced to provide full watershed descriptions of current and legacy pools of nutrients, including age, chemical profiles, nutrient gradients and stoichiometry, areal distributions, and transport processes. 


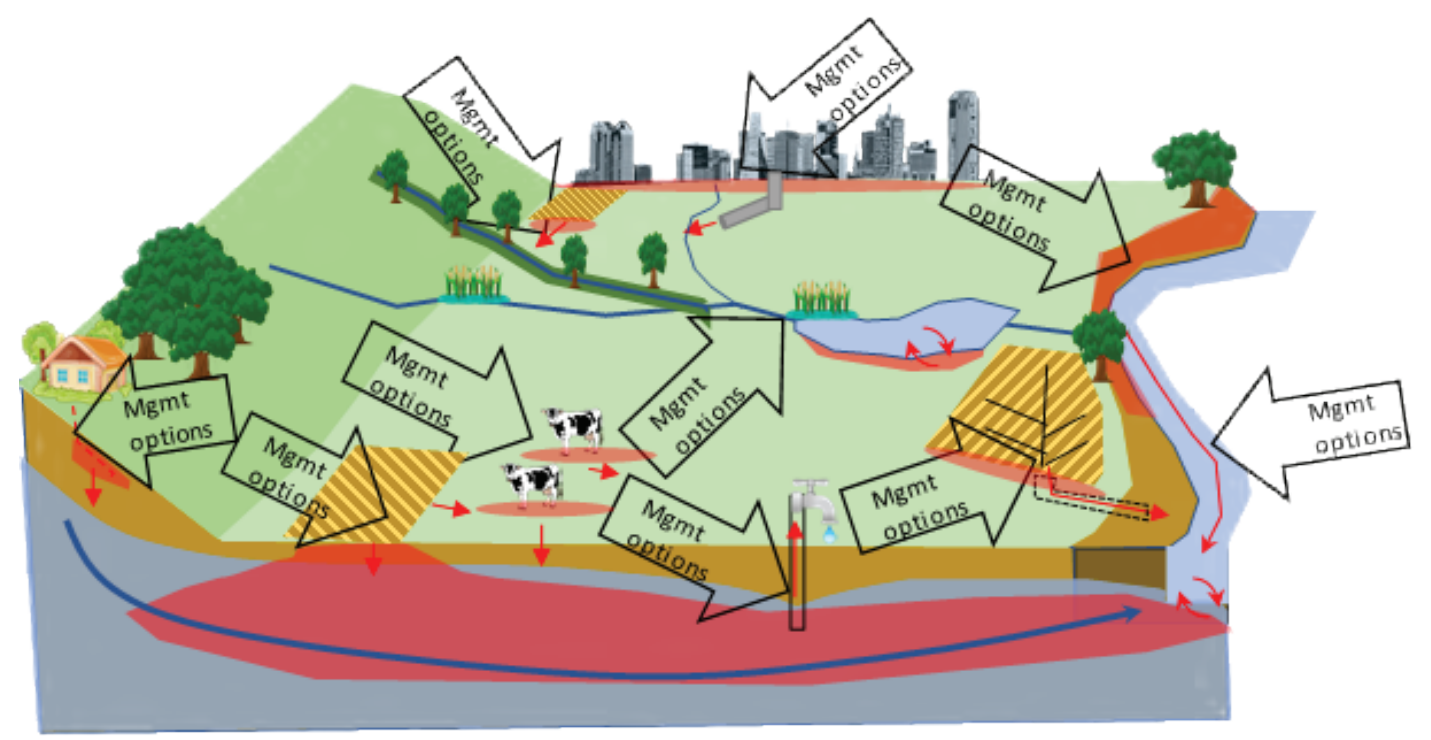

Figure 5.4. Conceptual model of legacy nutrient pools and their impact on water resources. (Red arrows represent nutrient transport/processing paths and red shaded areas represent nutrient pools). Different management options may be appropriate depending on the source of the nutrient pool and its mobility.

\section{Operational Implementation and Technical Considerations}

Evaluating water quality as a function of both current land practices and legacy nutrient sources will facilitate development of a full ecosystem approach to better forecast changes in the water quality in downstream receiving waterbodies. This information will help to inform decisions about where, when, and how to implement the most effective best management practices and stream restoration measures to improve water quality.

Datasets describing nutrient storage and processes that impact nutrient transport should be identified and catalogued for a specified area to allow for high performance computing and mapping. Identifying and translating these data to cloud optimized geo-tiffs, that could be readily displayed and queried, would improve both access and communication, as well as identifying geographic gaps in our understanding of nutrient pools, transport, stoichiometry, and bioavailability. Data gaps can be filled through field sampling by USGS and partner agencies. Researchers may need to partner with software developers to develop interactive maps to display nutrient pools and transport. Available data resources include ScienceBase, the National Water Information System and Water Quality Portal, and the National Cooperative Soil Survey Characterization Database. Existing watershed mapping tools that could be incorporated include SPARROW Mappers, SWATviewer, and Agricultural Conservation Planning Framework (ACPF) Tool.

\section{Longevity and Sustainability}

Legacy sources reflect an accumulation of excess nutrients in the environment, much of which was purposely applied in agricultural production. Consequently, it is logical that as new nutrient additions are minimized and responsibly managed that the potential mobilization of legacy nutrients may change. It is widely recognized that this is a long-term issue and will require long-term plans for management and assessment of the conditions. Quantifying these legacy nutrient pools is a first step; however, ongoing estimation of the magnitude of these pools in the context of new nutrient contributions, in-stream conditions, and receiving water-body conditions is critical to measuring how effective targeted actions are to reaching nutrient goals. Requirements to sustain this effort include:

- Infrastructure to monitor the size, age, and mobility of these legacy sources

- Monitoring of groundwater, in-stream, in-lake, and upland-soil legacy pools to focus and prioritize best management practices (BMPs) and riparian restoration.

- Modeling to assess status and predict changes as results of changing climate or management actions.

- Transfer of methods and results among multiple regional systems in the mid-continent, as well as those in other areas of the country where questions of water quality may include salinity and degradation of biocides. 
- Watershed mapping tools that are publicly served and regularly updated with new data and results, allowing managers and stakeholders to modify management plans as new data are provided.

Primary Contact: Tanja N. Williamson, tnwillia@usgs.gov

\section{NowCast+-Expanding eDNA Tracking of Pathogens and Invasive Species Using the NowCast Framework}

The Great Lakes NowCast is an existing interactive mapping product that displays daily predicted water-quality conditions for recreational areas in the Great Lakes Region; public health officials and resource managers determine the probability for elevated $E$. coli concentrations (indicating the potential presence of human pathogens) and, therefore, the need for an advisory or closure to protect the public from waterborne illnesses. By pairing up the existing NowCast framework with USGS streamgages and real-time eDNA capabilities, we can map out areas where pathogens and nonindigenous aquatic species are occurring.

\section{Demand and Relevance}

Local, state and federal partners have been using the NowCast system since 2006 to determine when E. coli advisories or closures should be issued using models to predict $E$. coli concentrations, as studies have consistently shown that elevated $E$. coli levels, especially from human/anthropogenic sources, relate to increased levels of waterborne illnesses (for example, gastroenteritis) in contaminated beach waters. This system provides a framework to build additional remote monitoring and forecasting of other human and wildlife (for example, bird botulism, an endemic problem in the lower Great lakes) pathogens, as well as non-indigenous species using DNA-based (eDNA) technologies. By incorporating eDNA sampling of species that pose a threat to public health, aquatic communities, and ecosystem services into established NowCast sites and USGS real-time streamgaging stations, we can map locations where these organisms pose the greatest threat; thus, remediation or restoration of degraded habitats can be better coordinated by expanding the existing NowCast system. Incorporating eDNA collection of non-indigenous species and pathogens at current NowCast locations, while adding additional Great Lakes sites and USGS streamgaging locations, will expand the forecasting and tracking of species that indicate a threat or pose the greatest threat to public health and aquatic communities such as E. coli, avian botulism (caused by Clostridium botulinum), grass carp (Ctenopharyngodon Idella), Dreissena spp. mussels, lampreys, milfoils (Myriophyllum spp.) and round goby (Neogobius melanostomus). The product of the expanded NowCast could be used by local, state, federal and academic agencies to provide data for decision making on topics ranging from non-indigenous species control to public health advisories.

\section{Improved Decision-Support}

Early detection of environmental threats is critical to improve stakeholder decision making. For instance, USGS researchers recently demonstrated the use of a robotic water sampler to collect, process, and preserve environmental DNA (eDNA) from water samples for near real-time stream assessments. The DNA from such collections offer opportunities for targeting multiple species or targets of interest: from microbial pathogens to aquatic invertebrates to fish species over a defined timeline. It is important to note that autonomous, robotic water sampling technologies represent the cutting-edge science in environmental sampling that can overcome the temporal and human (manual) resource demands often associated with traditional eDNA sampling. Thus, near real-time results can potentially be achieved for species of choice (single or multiple), as needed (that is, site- or location-specific monitoring; Asian Carp, dreissenid mussels, sea lamprey etc.). Additionally, the flexibility of eDNA methods allows for resource managers to effectively answer temporal questions that may arise at later dates, along with providing near real-time detection and monitoring of the current species of interest even when conditions are unfavorable for sampling. Because eDNA has broad applications, we can work with decision makers to identify needs/priorities (for example, high risk watersheds to identify threats in Western US).

With the increase of this much needed information which allows for improved decision making, expected impacts include direct funding for restoration of degraded habitats, improvements to infrastructure, implementation of best management practices (for example, alterations to shoreline to improve water circulation to reduce contaminant loadings at embayed beaches); citizen engagement (shoreline water quality, bird botulism; nuisance algae etc.); more effective early detection-rapid response actions. 


\section{Science Capabilities and Advancement Opportunities}

NowCast has been in operation since 2006 and has a total of 22 sites on Lakes Erie and Ontario. The USGS has strong cooperator ties with many local and state agencies who are collaborators on the NowCast. Additionally, the Wisconsin Department of Natural Resources has partnered with EPA and USGS to collect $E$. coli samples and map the results at another 310 sites within the Lake Michigan and Lake Superior watersheds. Michigan Department of Environment, Great Lake and Energy collects E. coli samples along their Great Lakes and inland beaches as well. The USGS also has thousands of real-time streamgaging stations throughout the Midcontinent Region.

Collection of eDNA or other biological samples (viruses, bacteria, nuisance algae and cyanobacteria, etc.) at selected streamgages by trained USGS personnel during their regular site visits requires little additional investment. USGS Science Centers have staff with experience utilizing robotic autosamplers and the expertise to combine real-time and high-throughput molecular data with environmental data (for example, water quality and weather) into an expedient data science pipeline to make predictions about species occurrence. Integrating predictive capability of NowCast water quality parameters (from streamgages) with eDNA studies provide opportunities to develop new predictive/forecasting models for current and emerging invasive species and integration with the USGS NAS database, which now reports eDNA results. Additional databases are also currently available using robotic and manual sampling, including Sepulveda (2020).

With the addition of the described methodology, biological data could be collected at sites with streamflow and water quality data for concomitant analysis and delivered using continuously updated maps showing distribution of species of interest in relation to area, time, water quality, season, etc. The use of machine learning algorithms can also be employed to estimate changes in species distribution as it relates to all other data collected.

\section{Operational Implementation and Technical Considerations}

The NowCast is currently operating as a standalone database and visual tool that is used by local, state and federal partners (fig. 5.5). It could be used as a baseline for further EarthMAP utilities and Use Cases. By incorporating additional tracking and methodology for environmental threats and utilizing existing USGS expertise, NowCast could expand to incorporate the entire Midcontinent Region and eventually the whole US and territories. Using existing data harvesters, NowCast is able to pull data from multiple sources including, but not limited to USGS, NOAA, and other online servers, which could be expanded to partner labs, universities, and state and local databases provided the data is available online.

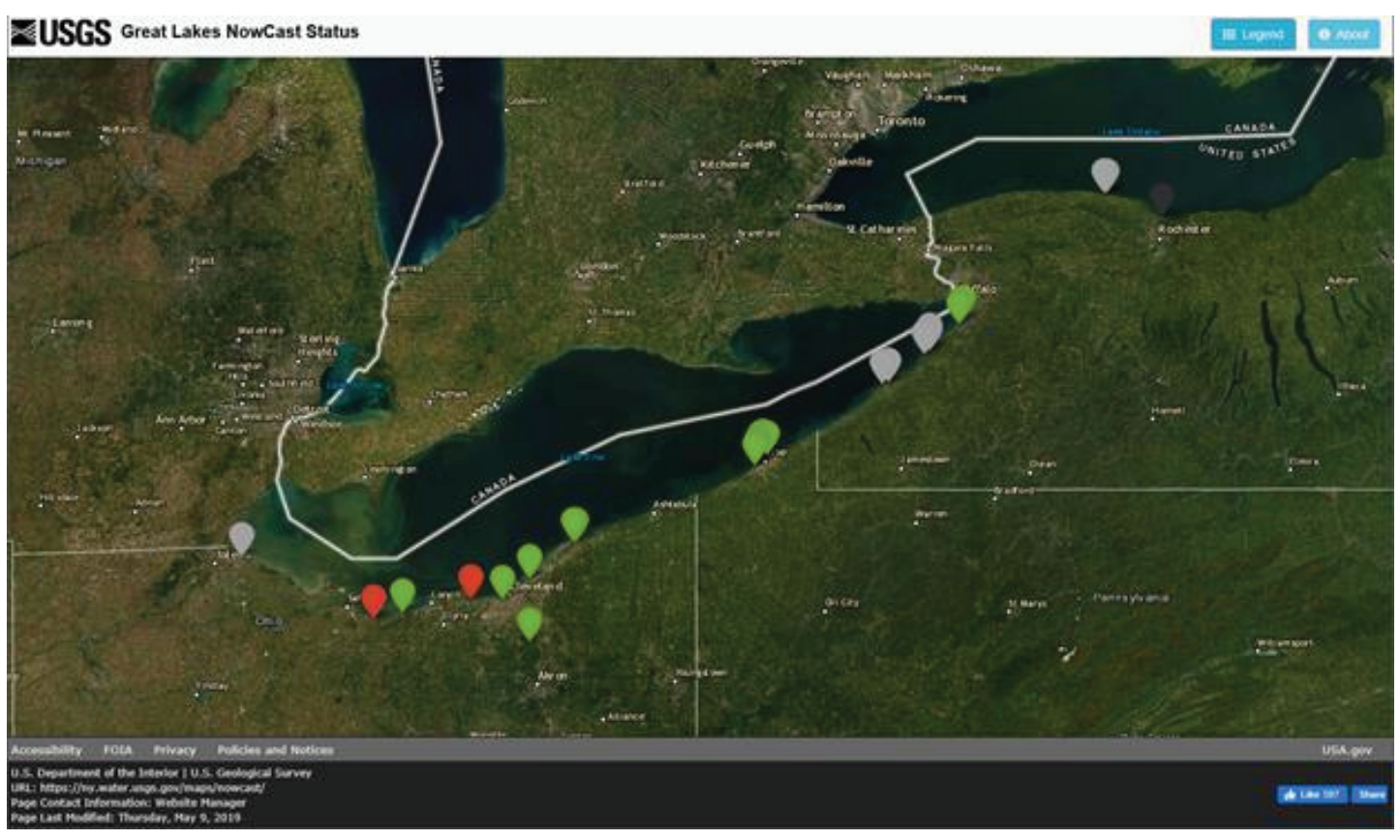

Figure 5.5. Great Lakes NowCast Public Portal—https://ny.water.usgs.gov/maps/nowcast/. The public portal currently shows the recreational water-quality conditions for NowCast sites within the Great Lakes Region. This versatile tool features a scalable map that allows for the addition of new sites nationwide to easily visualize water-quality or species-specific (eDNA) information consistent with U.S. Geological Survey (USGS) and stakeholder priorities. 
Autosamplers, including highly advanced systems such as the Environmental Sample Processor (ESP), provide opportunities to collect samples at predetermined times and can hold such samples in situ until they are picked up for further processing. Moreover, certain advanced ESPs have built-in capabilities for collecting and processing samples for pathogens and (or) their indicators, as well as harmful algal blooms (HABs). Such technologies can be transferred to invasive species based on location-specific needs. With automated sampling, we can better coordinate time of sampling (for example, catching spawning events; disease outbreaks), reduce manual training, and sample processing needs. Because eDNA has broad applications, we can work with decision makers to identify needs/priorities (for example, high risk watersheds to identify threats in Western US).

Bringing together the database and visualization capabilities of NowCast and the remote collection capabilities of the USGS requires incredible computing power, but when implemented properly would provide a robust and user-friendly web-based tool that is adaptive to the needs of the stakeholders.

\section{Longevity and Sustainability}

The eDNA technologies are evolving at a rapid rate. The Use Case summary provided here may easily be transferred to novel and emerging invasive species, pathogens or microbes, consistent with USGS priorities and partners' needs. Some western states are already using existing streamgages for biological threats which can be further expanded to meet regional or national priorities. The use of eDNA for detection of invasive species, pathogens or microbes in conjunction with water quality parameters from streamgages will identify hotspots of potential threats while protecting human health and identifying sensitive aquatic native/endangered species and habitats within the Nation's lakes and waterways. There will continue to be emerging threats to human health and natural resources; the NowCast tool is equipped to be the powerful early detection system for these novel threats.

Primary Contacts: Amie Brady, amgbrady@usgs.gov; Amanda Bell, ahbell@usgs.gov

\section{Northeast Region}

\section{An Integrated Framework for Assessing Bird Populations to Better Understand Drivers of Population Change and Project Future Outcomes}

We propose to develop an integrated modeling framework for assessing and visualizing national and regional bird population abundance and trends to inform the management of North American bird species, supporting the regulatory and management decision making of external partners (for example, USFWS and State agencies), and strengthening the ties between government and nongovernment collaborators.

\section{Demand and Relevance}

Following a recent study estimating greater than 3 billion birds lost since 1970, there has been a call for the scientific community to collaborate broadly across geographic locations and jurisdictions to identify the drivers of declines in bird populations throughout their range, and to forecast changes in population size and distribution, given uncertainty in future environmental and management scenarios. The North American Breeding Bird Survey (BBS) is the primary source of the population change information that went into the 3 billion birds assessment, and the BBS is also a primary dataset for many other modeling activities. New opportunities exist for developing integrated models for BBS and eBird, as well as other datasets (that is, Christmas Bird Counts, Alaska Landbird Monitoring Surveys, Integrated Monitoring in Bird Conservation Regions) that can (a) enhance the value of the BBS for estimation of population change (it's primary historical use); (b) expand inference from the BBS to allow for estimation of change on and off roadsides; (c) allow for estimation of total abundance; and (d) allow for development of spatially-explicit models that directly connect change in land use and management to changes in bird populations. Integrated models that allow for spatially-explicit integration of eBird and other datasets collected with traditional survey designs provide a critical step forward for meeting the spatial information needs for bird conservation and management over the next decade. 
The bird conservation community has devoted a huge amount of effort into using BBS and other datasets to inform management at local scales via spatial models. The "Avian Knowledge Network" is one example of this, and multiple modeling efforts that Partners in Flight and the North American Bird Conservation Initiative have sponsored, are also part of that effort. These efforts have been limited in scale and applicability by available data, in that BBS has issues that limit it as an omnibus spatial dataset (for example, roadside frame). While eBird, on the other hand, has recently provided spatially-explicit maps, but they are descriptive rather than providing models that facilitate management decisions by producing predictions of management actions. We propose extending the geographic and operational scope of a recently developed integrated modeling framework to leverage eBird and Breeding Bird Survey (BBS) data, thereby contributing to the BBS mission of remaining a leading source of data for bird management and conservation, supporting management and regulatory decision making of external partners (USFWS, Canadian Wildlife Service, National Park Service, Forest Service, and Bureau of Land Management, Mexican National Commission for the Knowledge and Use of Biodiversity), and strengthening the ties between government (USGS, FWS, State agencies) and nongovernment (that is, Cornell Lab of Ornithology, National Audubon Society, Partners in Flight) collaborators. This framework will expand the scope of taxa and geographic locations (for example, North Atlantic-Appalachian and nationally) for which we can make reliable inference and improve management decision making with more precise estimates of population trends for species of management concern. The integrated model can be used to support annual decision making by state and federal agencies, and to forecast changes in bird populations over longer time scales in response to climate and land-use change. The science and products developed from this effort align with the priorities of DOI (Create a conservation stewardship legacy second only to Teddy Roosevelt), the USGS Ecosystems Mission Area (species at risk; migratory birds; and, modeling and forecasting species distribution, abundance, and activity), and the North American Breeding Bird Survey program (Advance research on model development, including integrating complementary data, to better meet stakeholder needs; improve availability, utility, and visual appeal of analytical products; and, develop stronger partnerships with other organizations to enhance the mission of the BBS).

\section{Improved Decision-Support}

We recently developed an integrated population model, in collaboration with the Cornell Lab or Ornithology and FWS, using eBird and traditional aerial surveys to estimate the 2020 breeding population size of mottled ducks on the western Gulf Coast (fig. 5.6). The COVID-19 pandemic disrupted aerial surveys, however, FWS still had a critical need for population size estimates to make regulatory decisions. Our estimates are being used by the Service Regulations Committee to inform the current and future management of this species. This Use Case will improve the decision-making process for external cooperators (FWS, State agencies, BLM, USFS) by a) increasing the geographic and operational scope of recently developed integrated population models, b) improving precision of bird population estimates, and c) forecasting at multiple temporal scales relevant to the Department of Interior. This framework will be used to conduct population assessments at multiple spatial scales (that is, regional to continental), and for priority species identified by external partners. For example, USFWS has identified waterbirds as a guild that has typically received less research attention, yet has many species experiencing large declines in population size throughout their range. Forecasts on an annual scale will support management of species regulated by USFWS, and forecasts made on decadal scales will be relevant to understanding the potential impact of climate and land-use change on species of concern.

\section{Science Capabilities and Advancement Opportunities}

The Use Case relies on the extensive historical data provided by the BBS program as well as more recent community science data provided by the eBird program, and auxiliary data which drive bird population and distribution dynamics such as land-use change data from MODIS and NLCD. We bring these data resources together using modern methods of integrated modeling, which control for specific sources of bias and error in each of the contributing programs. This project will rely on existing USGS expertise in model development, data integration, and ecological forecasting. Scientists with USGS will provide expertise in using BBS data and external cooperators with the Cornell Lab of Ornithology will support the use of eBird. Guided by preliminary model development, this effort will extend the operational and geographic scope of integrated population models for a) conducting population assessments at regional and continental scales to guide annual decision making by USFWS, b) forecasting the impact of changing climate and land use on bird populations of conservation concern, and c) filling critical knowledge gaps for species that are of concern but have been relatively under-studied. This effort also has the potential to support and advance partnerships with other government agencies (that is, NPS, USFS, and BLM. For example, the NPS Inventory and Monitoring Program conducts annual bird population monitoring. Integrating these data with eBird and BBS will support population assessments and regulatory decision making within national parks. 


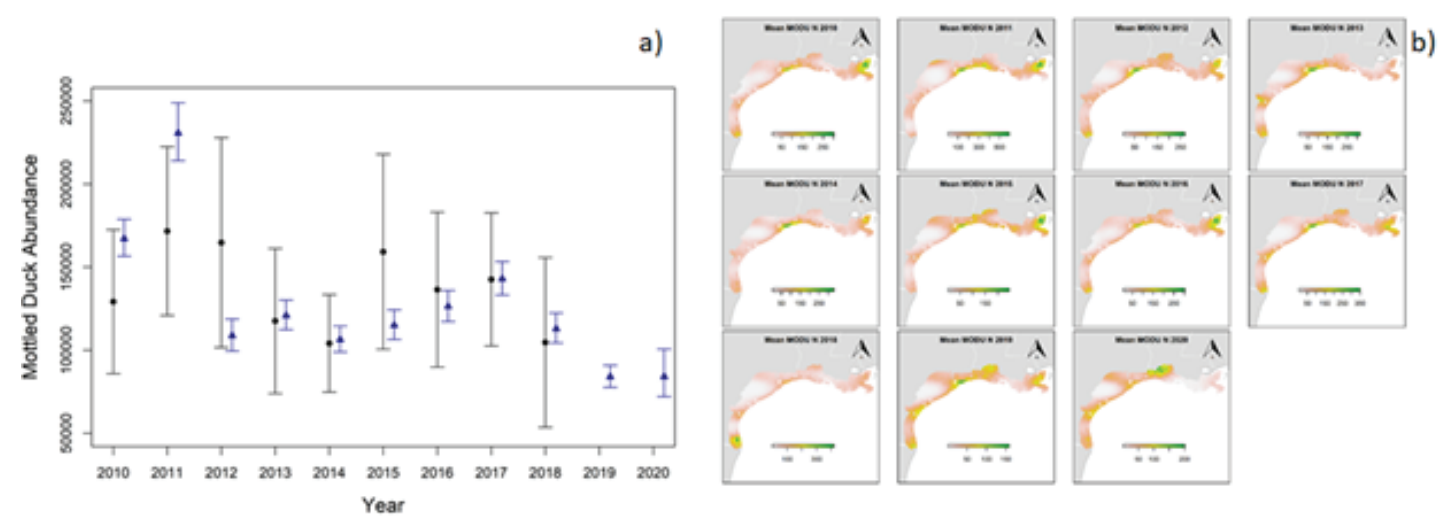

Figure 5.6. We developed an integrated model to inform management decision-making for mottled ducks in the Gulf Coast Region. This critical forecasting tool was developed to fill an information gap created by the COVID-19 (Novel coronavirus disease-2019; SARS-CoV-2) pandemic that resulted in the cancellation of operational Fish and Wildlife Survey (FWS) surveys at a critical time in the monitoring cycle. $A$, Total abundance each year (blue triangles=posterior means, blue lines=90-percent credible intervals) from 2010 to 2020 from the integrated model fit to eBird checklists and aerial surveys. Black circles and lines indicate mean and 90-percent confidence intervals (calculated as $\pm 1.96 \mathrm{SE}$ ), respectively, from FWS reports using the VCF method. Estimates using the VCF method were not available for 2019 or 2020; and B, Spatial maps of predictions showing estimated mean mottled duck abundance in each $6 \times 6 \mathrm{~km}$ grid cell estimated from the integrated model from 2010 to 2020.

\section{Operational Implementation and Technical Considerations}

USGS currently performs annual population assessments of migratory bird populations from the North American BBS program and the technical capacity to initiate the Use Case exists at Leetown/Patuxent. A number of on-going collaborative efforts between USGS and FWS provide annual assessments of species managed for take and this partnership is a key element of the proposed Use Case. Moreover, by advancing collaborations with the Cornell Lab of Ornithology we have established a multi-organizational team of experts in bird conservation and data science laying the technical groundwork and possessing the expertise to advance delivery of the Use Case. Key to initiation of the project is bringing together the data resources of the North American BBS program, maintained by the USGS, and those of the eBird program operated by the Cornell Lab of Ornithology, which has been demonstrated in one ongoing partnership between USGS, FWS and the Cornell Lab of Ornithology. Operational implementation and sustainability of the initiative will require additional staff resources in the form of one or more data scientists on staff associated with the BBS program.

\section{Longevity and Sustainability}

For more than 50 years, the North American Breeding Bird Survey has helped keep common birds common by providing breeding population data for more than
500 species and serving as a quantitative cornerstone for avian conservation and management in the U.S. and Canada. Integrated models, that allow for spatially-explicit integration of the extensive eBird dataset and other datasets based on traditional survey designs, provide a critical step forward for USGS to continue meeting the spatial information needs for North American bird conservation and management. There will be an increasing need for short term predictions and long-term forecasts of bird population status by the bird conservation community as bird populations are increasingly affected by climate and landuse change. EarthMAP represents a unique opportunity as it provides a framework for integration of USGS land, water, and geological mapping products with biological datasets. Strong partnerships established between the entities (that is, USGS BBS, USGS Science Analytics and Synthesis, Cornell Lab of Ornithology) maintaining and curating data, developing modeling frameworks and presenting output to decision-makers and the public will ensure the sustainability of this project long-term. A successful project will be measured by the use of population assessments by external cooperators (for example, FWS, USFS, NPS, BLM, PIF [Partners in Flight], NABCI [North American Bird Conservation Initiative], and state wildlife agencies) in management-decision making and filling critical knowledge gaps for species of concern.

Primary Contact: Andy Royle, aroyle@usgs.gov; Paige Howell, phowell@usgs.gov 


\section{Integrated Monitoring, Analysis, and Modeling for Enhanced Predictive Capability of Nutrient Loads from the Connecticut River Basinto Long Island Sound}

The New England Water Science Center will use an innovative integrated monitoring, analysis, and modeling framework to enhance predictive capability of nutrient loading from the Connecticut River Basin to Long Island Sound. A key focus of the initiative will be the incorporation of dynamic (seasonal) transport processes and nonpoint-source loading of nutrients from groundwater into the modeling and analysis framework.

\section{Demand and Relevance}

Long Island Sound provides social, recreational, and commercial benefits to millions of residents in Connecticut, New York, and surrounding States. However, four centuries of settlement and industrialization in the Sound's watershed have led to widespread low dissolved oxygen, toxic contamination, pathogens, and floating debris. Seventy percent of all freshwater entering the Sound is from the Connecticut River, New England's largest river system (fig. 5.7). The Connecticut River drains 11,250 $\mathrm{mi}^{2}$ in five States and Quebec and flows

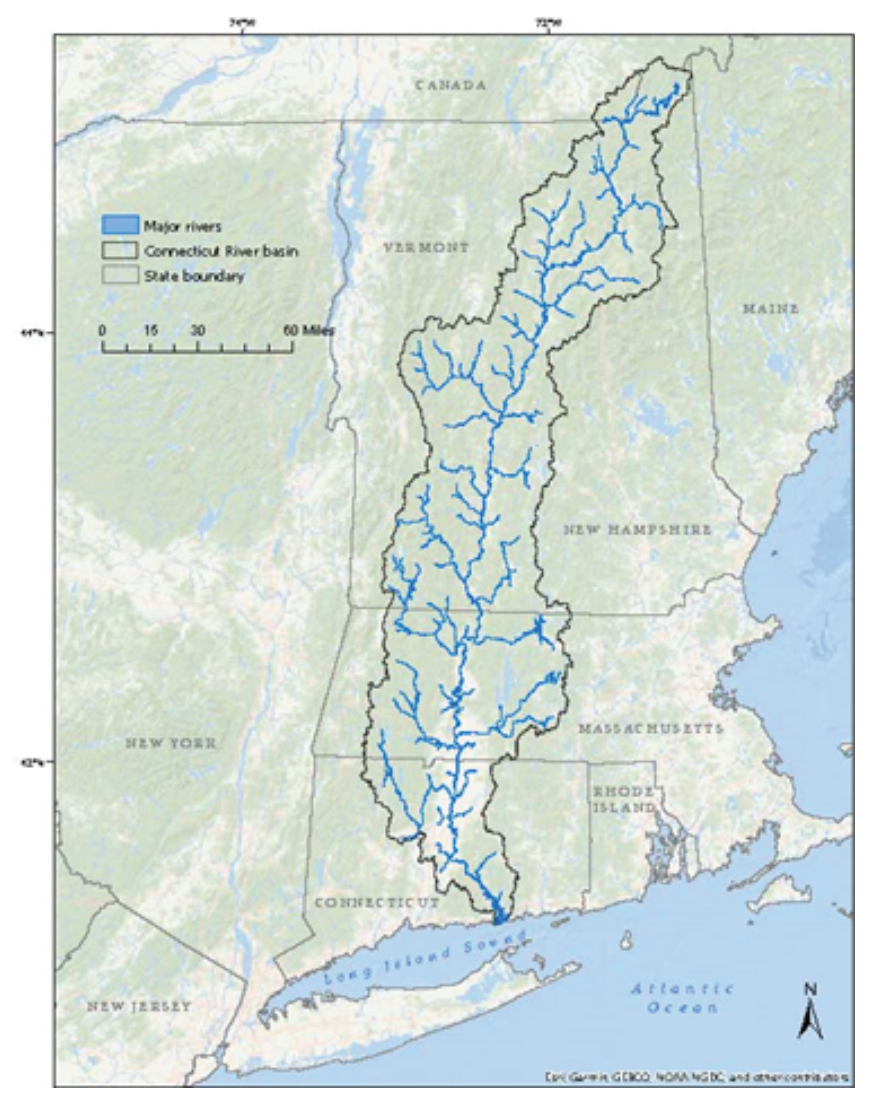

Figure 5.7. The Connecticut River Basin and Long Island Sound.
410 miles through forests, rich agricultural lands, and urban centers. The river's watershed provides important habitat for a diversity of fish, wildlife, and plants, and is home to several federally threatened and endangered species including the shortnose sturgeon, piping plover, and dwarf wedgemussel. Nutrient loading (primarily nitrogen) from point and nonpoint sources within the Connecticut River Basin has been a priority environmental concern for Connecticut, Massachusetts, New York, and Long Island Sound stakeholders for many years. In response, the USGS New England Water Science Center, with collaborators at the USGS New York Water Science Center, Woods Hole Coastal and Marine Science Center, Leetown Science Center, and the Land Change Science Program, has been engaged in monitoring, modeling, and understanding of watershed processes that influence water quality and ecosystem services within the basin and forecasting environmental outcomes from potential land-use changes and pollutant-management actions.

The Center operates 65 streamgages, 19 temperature gages, and 17 water-quality stations in the basin. This data network provides critical real-time information on nutrient conditions in the watershed and nutrient delivery to the Sound. The Center also conducts nested environmental assessments and modeling studies that range from watershed-scale field research at the Sleepers River Watershed in Vermont to regional-scale modeling of nitrogen, phosphorus, and carbon throughout the basin. These monitoring, analysis, and modeling activities are done in partnership with the Connecticut Department of Energy and Environmental Protection (CTDEEP), Massachusetts Department of Environmental Protection, U.S. Environmental Protection Agency, the Long Island Sound Study, and many others. The Center has a robust program of stakeholder engagement in the basin and continually works to understand and address stakeholder needs.

In collaboration with its several partners, the New England Water Science Center will develop an integrated monitoring, analysis, and modeling framework that advances the understanding of nutrient sources, watershed transport processes, and trends within the Connecticut River Basin and the predictive capability of nutrient loading to Long Island Sound by explicitly incorporating dynamic (seasonal) transport processes and nonpoint-source loading of nutrients from groundwater into the modeling and analysis framework.

\section{Improved Decision-Support}

Nutrient management in the Connecticut River Basin has been focused on reducing point sources of contamination to rivers within the watershed yet monitoring at USGS streamgaging sites indicates that nonpoint sources are a substantial part of the total nutrient load to the river. Although nutrient loading from groundwater discharge has been shown to be an important, long-term nonpoint source to many freshwater and marine ecosystems, groundwater contributions to the Connecticut River have not been quantified. 
A fundamental goal of EarthMAP is to provide actionable intelligence of direct value to USGS partners, stakeholders, and the public. To accomplish this goal, we will engage our collaborators and stakeholders early in the project-development process, as well as throughout the full life of the project, to ensure that the data, scientific information, and predictions that result from this EarthMAP initiative meet their resource-management needs. The monitoring and modeling framework developed as part of this initiative will provide the basis for improved seasonal and long-term forecasts of land-use changes, climate change, and management actions that address both point and nonpoint sources of nutrient loading. The knowledge gained from this predictive capability will provide managers much needed guidance on how, where, and when to conduct mitigation measures aimed at improving water quality throughout the watershed, as well as information needed to understand and manage the impacts of nutrient loading to freshwater and marine wildlife, fisheries, vegetation, and dynamics of harmful algal blooms.

\section{Science Capabilities and Advancement Opportunities}

The Center's EarthMAP initiative will consist of two key components that will support each other and provide feedbacks for overall system improvement: a streamflow and water-quality monitoring network and an integrated modeling framework.

(A) Monitoring Network: The Center has operated a surface-water quality network to monitor nitrogen loading to Long Island Sound in cooperation with CTDEEP since 1973, shortly after enactment of the Clean Water Act. Through the years, the Center has continued to upgrade the network with novel monitoring methods and state-of-the science data analyses to determine daily estimates and long-term trends in nitrogen concentrations and loads to the Sound (for example, Mullaney, 2016), which provide important dependent variables for calibration of the transient models.

(B) Modeling Framework: A comprehensive, integrated modeling framework will be developed to support seasonal to decadal predictions of nutrient loads to Long Island Sound from the Connecticut River Basin. The initial framework will include the following components, which will be expanded to include models to represent ecosystem dynamics:

Dynamic SPARROW models: Two sets of SPARROW

models have been developed to aid in the understanding of the long-term sources, fate, and transport of nitrogen, phosphorus, and suspended sediment across the Connecticut River Basin (Moore and others, 2004; Ator, 2019). SPARROW-SPAtially Referenced Regression on Watershed attributes - is a hybrid statistical and mechanistic model for estimating the load of a target constituent moving through the landscape. SPARROW was initially designed to simulate long-term, steady-state conditions (Schwarz and others, 2006). Recently however, the SPARROW methodology has been extended to simulate dynamic (transient) conditions with an initial emphasis on seasonal time periods (Greg Schwarz and Richard Smith, USGS, written commun., 2020). The New England Water Science Center has contributed to the testing of the new dynamic SPARROW modeling approach by modifying the original, steady-state model developed by Moore and others (2004) to simulate nutrient transport and loads for 35 seasons extending from winter 2001 through summer 2009 (Richard Moore, USGS, written commun., 2020). The models of Moore and others (2004) and Ator (2019) will form the basis for new, dynamic SPARROW models developed for the basin.

Groundwater/surface-water modeling: The Center will use a cutout of the National Groundwater Model (NGWM; Zell and Sanford, 2020) to simulate transient groundwater flow, groundwater/surface-water interactions, and nitrogen loading from groundwater discharge to streams. The NGWM is an innovative, MODFLOW 6 based model of the shallow groundwater system for the contiguous United States. Detailed simulation of the basin's groundwater system is needed to accurately represent transport of nonpoint sources of nitrogen throughout the basin, as well as the time lags that occur between the entry of nitrogen into the soil zone and discharge of groundwater nutrient loads to streams and coastal waters. Subsurface nitrogen transport will be simulated using methods developed by Center staff as part of an ongoing regional groundwater-modeling project along the north shore of the Sound (Janet Barclay and John Mullaney, Fall 2020 AGU abstract). Model-simulated groundwater discharge and nitrogen loads will be directly coupled with the dynamic SPARROW models through a consistent surface-water drainage network.

Landscape modeling: The Forecasting Scenarios of land use (FORE-SCE) model will be used to reconstruct historical landscapes in the basin and to simulate future landscape change. FORE-SCE is designed to leverage USGS land-change monitoring data using information from the Earth Resources Observation and Science (EROS) National Land Cover Database (NLCD) and Land Change Monitoring, Assessment, and Projection (LCMAP) projects (Sohl and others, 2019). LCMAP uses a national-scale, high-resolution (30-meter) parcel-based modeling framework to simulate past, present, and future landscape change. 


\section{Operational Implementation and Technical Considerations}

The Center will work closely with the USGS Water Resources Mission Area (WMA) developers of the dynamic SPARROW software and NGWM to ensure mutually beneficial feedback among the development and application teams. Moreover, the Center will design the modeling framework in consultation and partnership with the WMA's Integrated Water Prediction Program and biologists with the USGS Leetown Science Center (Ecosystems Mission Area). Because of the computational demands of the proposed integrated modeling system, it is anticipated that the system will need to be deployed on one of the USGS high-performance computer systems, such as the Denali system housed at the Earth Resources Observation and Science (EROS) Center in Sioux Falls, South Dakota.

\section{Longevity and Sustainability}

Because nutrient enrichment of surface waters from both point and nonpoint sources is such a widespread environmental concern and because the SPARROW and MODFLOW modeling systems have been applied extensively across all geographies of the United States, it is anticipated that the integrated modeling system developed as part of this EarthMAP initiative will have substantial transferability (for example, basins contributing to the Great Lakes). Moreover, the long history and institutional knowledgebase of the SPARROW and MODFLOW modeling systems suggests that they will remain viable modeling environments within the USGS for the foreseeable future.

Primary Contact: Johnathan R. Bumgarner, jbumgarner@usgs.gov

\section{Observation, Assessment, Prediction, and Operationalizing of a Regional Analysis of Sediment and Co-Contaminate Flux and Sediment Sources in the Delaware River Basin}

Effective management of sediment pollution, associated co-contaminants, and remediation of negative ecological end points (habitat destruction, harmful algal blooms, etc.) requires understanding not just the amount of sediment and co-contaminates being delivered, but also understanding the location of sediment sources within a system and understanding how co-contaminants are related to those sources. The Sediment Use Case, described here, lays out a new vision integrating measurement, assessment, and prediction in un-measured areas for delivery of sediment and co-contaminant flux and sediment sources in watersheds across the United States. This information will be useful to a broad array of resources managers from drinking water purveyors, to state environmental agencies, to industrial water users, as well as wildlife managers and sportsman.

\section{Demand and Relevance}

Sediment is a major pollutant nationally, and in the Delaware River basin (DRB), affecting reservoir storage and degrading aquatic habitat, as well as a transport vector for delivering attached contaminants to downstream water supply and ecosystems. Sediment and sediment-associated constituents can contribute substantially to water-quality impairments and infrastructure problems that can lead to water-availability impairments. Infrastructure problems associated with sediment include reservoir sedimentation, drinking supply intakes, erosion of critical infrastructure, and navigation channel maintenance. Ecological problems associated with sediment include light attenuation, burial of channel substrate, reducing primary productivity, impairment of benthic macroinvertebrates, clogging of fish gills, altering oxygen demand, and effect on egg gestation. Identifying the sources of this sediment and quantifying its flux in streams is information needed by management agencies to alleviate this sediment problem.

In general, the two main sources of sediment are upland erosion sources (soil erosion from various land uses) and streambanks. If sediment-source results indicate that streambanks are an important source of sediment, modeling sediment transport and delivery must include this as a term in their models. Sediment-trend analysis should include identifying channel change over time by either human or natural causes. Unfortunately, many of the models used to estimate sediment flux do not include a robust streambank erosion process. In the Chesapeake Bay watershed, over a billion dollars is spent each year on restoration, and the efficiency and effectiveness of that spending directly follows from the availability of the models and best science to predict pollutant sources in the landscape. 
The major stakeholders interested in managing sediment pollution include industrial, agricultural, and municipal, and commercial resources managers who are tasked with treating intake water or preventing sediment movement related to their particular water-uses. Natural-resource managers including agencies that managed habitat, wildlife, fishes, and ecosystem responses (that is, eutrophication) are particularly interested in understanding the role of sediment in impairments and identifying the most effective management strategies and locations for reducing sediment pollution. To that end, the Sediment Use Case expects involvement of the USGS Water Resources Mission Area (WMA), USGS Ecosystems Mission Area, the USGS Integrated Water Availability and Assessments (IWAAs) program, the Integrated Water Prediction (IWP) program, and the Next Generation Water Observing System (NGWOS) program, all having interest in understanding sediment pollution for Integrated Water Science in the Delaware River Basin. The NGWOS program has invested resources during Federal Fiscal Year's 2020-22 to pilot improved monitoring, rapid development of site-specific continuous models of sediment concentration, and landscape \& channel sourcing of sediment in the DRB. In addition, funding from Ecosystems Mission Area, the Penn Foundation, and Smithsonian Institution is supporting an effort to improve modelling of floodplain and channel sources of sediment using the Floodplain and Channel Evaluation Tool (FACET, a toolkit to automate stream geomorphometry estimation) and USGS Chesapeake and Delaware Floodplain Network (CDFN, a measurement and modeling effort to predict streambank erosion and floodplain deposition).

Through the current work of NGWOS, Sediment Use Case envisions NGWOS-IWAA-IWP working together to monitor, assess, improve, and operationalize sediment prediction models over a large geographic extent (such as the DRB, the NAAR[the North Atlantic-Appalachian Region], or even larger regions of the U.S. depending on the extent of data collection). If the FACET-CDFN model can be improved in these ways, with closed sediment budgets, refined sediment sources, ancillary measurements of nutrients, bacteria and other sediment associated contaminates then FACET-CDFN can serve to feed and improve the accuracy, precision, and spatial extent of SPARROW models, upland sediment models, and machine learning tools under development in IWP. These goals align with DOI Mission Area goals and related USGS goals for conserving land, water, species, and habitat; managing DOI water storage and delivery; and informing land-use planning processes for public use and access with decision support tools. The goals of this Sediment Use Case also align with USGS WMA goals to provide data to society about the quality and quantity of water, improved understanding of processes (sediment transport) that determine water availability and predict changes in the quality or quantity of water related to external factors.

\section{Improved Decision-Support}

The Sediment Use Case that we are proposing would lead to improvements of sediment flux estimates within streams (erosion and deposition) and improved understanding of sediment sources in the DRB. The results of this study will meet WMA's objectives of understating and predicting sediment flux from source to sink. Currently, our team is engaged with the Delaware River Basin Commission (DRBC), the Chester County Water Resources Authority (CCWRA), the USGS Office of the Delaware River Master (ODRM), the USGS WMA, the USGS Integrated Water Science team for the Delaware River Basin (NGWOS, IWAAs, IWP), four USGS Water Science Centers (Pennsylvania [PA], New Jersey [NJ], New York [NY], South Atlantic [SA]), Stroud Water Resources Institute, the Penn Foundation, and several academics from the University of Delaware and the University of Minnesota. We are currently working with EPA to incorporate our predictions of streambank and floodplain flux of sediment, $\mathrm{N}$, and $\mathrm{P}$ into their next-generation regulatory model for the Chesapeake. Using improved reach-scale flux predictions and sediment source information, managers will be able to better predict problem areas, direct best management practices, more effectively treat water for public consumption, and aid in the restoration of ecological impairments.

\section{Science Capabilities and Advancement Opportunities}

Currently, NGWOS is assessing the rapid development of fluvial suspended sediment surrogate models using continuous turbidity or acoustic backscatter measurements. By deploying and selectively sampling based on streamflow statistics and turbidity/backscatter measurements robust predictions of suspended sediment concentration can be developed in less than 18 months. These predictions are on a 15-minute or shorter timestep and lead to highly accurate computations of hourly, daily, monthly and annual flux. These flux estimates can be used to greatly improve other models which attempt to estimate sediment in un-monitored areas.

Sediment fingerprinting is a method that compares the radiochemistry of upland, flood-plain and channel sediment sources to suspended sediment in streams using radionuclides common in rainfall, allows discrimination of the likely source (topsoil, streambanks, channel bed sediment). This radiochemistry can also provide a relative age of the sediment. If sediment is relatively young (that is, less than 5 years) then monitoring programs may show a decrease in sediment related to these management actions. If sediment is older (greater than 10 years), it may take longer until we see a reduction in sediment from these older sources. Combining sediment source analysis with age dating, provides managers and modelers with data need to control and understand sediment flux. 
The proposed statistical model will build upon the CDFN model (https://www2.usgs.gov/water/southatlantic/ projects/floodplains/) developed by Greg Noe and others using monitoring sites in the DRB and FACET model. Measurements of dendrochronology, field surveying, and sediment physio-chemistry were used to calculate changes in floodplain deposition and streambank erosion over time to create a quasi-sediment budget for each site. FACET incorporates high resolution airborne LiDAR to estimate channel and floodplain morphology, and along with characteristics of the upstream drainage area, was used to extrapolate the monitoring station results to the entire DRB. Statistical analysis including random forest regression (a form of machine learning) was used to develop statistical models of sediment flux with predictions of floodplain and streambank flux and geomorphic change for each NHDPlusV2 reach. By comparing floodplain and streambank fluxes (stream valley sediment fluxes) to SPARROW prediction of suspended sediment export from the catchment, a residual (other) sediment source was calculated using mass balance. The size of the modeled streambank vs. residual sediment source for any given reach estimates the contribution of channel vs. uplands to sediment transport. By expanding to monitoring stations across the DRB (or other IWS basins), the spatial resolution and accuracy of FACET-CDFN will be improved in new geographic areas. New reach-scale flux estimates could be used to improve SPARROW models by providing a new prediction variable (FACET flux).

This Sediment Use Case in the DRB will develop a scalable approach that can be implemented anywhere where appropriate fluvial sediment and sediment sourcing data is being collected. IWS basins (DRB, Upper Colorado, Illinois, and future basins) offer the opportunity to test not only the science, but the operation of NGWOS-IWAAs-IWP coordination. Scientifically, rapid sediment surrogate models from continuous monitoring, sediment fingerprinting and age-dating, FACET, random forest, and SPARROW models will improve the accuracy, timeliness, and spatial extent of sediment flux estimates and sources in unmonitored areas across the country. This sediment information could then be used directly by resource managers or be further incorporated into regional or national models to improve estimates of water availability.

\section{Operational Implementation and Technical Considerations}

Currently, the USGS has the technical capacity, interest, appropriate structure (NGWOS-IWAAs-IWP), and existing models and tools to operationalize a sediment flux and source workflow from a pilot in the DRB. Once the pilot in the DRB is complete, the developed template can be used in each other IWS basin across the country by gradually improving and standardizing the linkages between observation, assessment, and prediction of sediment flux, sources, and age. With advancements in real-time monitoring, improved workflows for sediment surrogate flux estimation, assessment of sources, geomorphic changes, high resolution LiDAR coverage, dendrochronology, FACET, and machine learning we have the tools to predict sediment flux more accurately and partition the relative sources (upland, floodplain, in-channel; ag, urban, storm water, etc.). However, the DRB Sediment pilot is currently under-resourced. We need resources from the IWAAs and IWP programs and more resources from the NGWOS in our pilot study for complete data collection, data assessments, prediction validation tests, and improvements to existing predictions. Effective delivery of this information could be done as a static data service, or more usefully, as a national web service that could deliver estimates of sediment sources, and real-time (at a daily or lower) estimates of sediment flux and apportionment of sediment sources on an annual or lower time-step (if applied to active sediment data stations). Data archives, sediment flux station site-specific models, and FACET meta data can all be served in a traceable format to the public from this same web service. By establishing a network of long-term monitoring locations through the NGWOS program, where continuous flux is estimated, routine drone LiDAR surveys and geomorphic measurements are conducted, and spatial variability is measured with synoptic observations we can create a data pipeline to feed assessment and prediction of sediment, at reach-scale, across the country. Data would be processed by the IWAAs and information products specific to local and regional needs can be delivered to local stakeholders, and IWP can establish computation pipelines involving FACET-CDFN, random forest, and SPARROW to provide highly accurate predictions of sediment \& co-contaminant flux and sediment sources. 


\section{Longevity and Sustainability}

By generating this template and applying it to existing and new IWS, in less than 10 years we could have vastly improved sediment flux estimations, identified sediment sources for resource managers, and improved on the predictive capability of national, regional, and local water models. The coordination of resources across USGS Water Resources Mission Area programs, along with Ecosystems Mission Area expertise, and the scaling of pilot projects over time - potentially to operational scales in new IWS basins - would help to ensure the sustainability of the Sediment Use Case. Once the workflow, data requirements, appropriate assessments, and data flow for prediction are established, sustainability would require data scientists and IT infrastructure to serve information at various spatial and temporal scales. This technique should be largely adaptable to different IWS basins, and also to interested science centers to support stakeholder needs. Potential problems could exist with computational power for proper scalability but should be feasible with existing cloud infrastructure. Model predictions should be approved at the work-flow level, and reproducible data pipelines should be used to expedite data delivery in a timely manner. Metrics of success should be evaluated on references to our data and hits on our data services.

Primary Contact: Joe Duris, jwduris@usgs.gov

\section{Integrated Multidisciplinary Science to Inform Habitat Conservation and Management Efforts for Fish and Wildlife in the Delaware Watershed}

Signed into law in December 2016, the Delaware River Basin Conservation Act of 2015 directed the Secretary of the Interior to establish a nonregulatory Delaware River Basin Restoration Program working through the Director of the USFWS and coordinating with Federal, State, joint venture partnerships and other public agencies and organizations with authority for planning and implementation of conservation strategies in the Basin. The multidisciplinary USGS science team would enhance the value of this effort through provision of integrated ecosystem science leveraging the Patuxent Wildlife Research Center/Leetown Science Center (PWRC/ LSC) expertise and research on the Chesapeake Bay to apply similar approaches in the Delaware River Basin (DRB) through the EarthMap Initiative.

\section{Demand and Relevance}

Similar to the Chesapeake Bay, research in the Delaware River Basin has focused on improving water quality with limited research on living resources and ecosystem science. This limited research has focused on the population trends of key species, such as Atlantic Sturgeon, white perch, striped bass, weakfish, American eel, American shad, brook trout, blue crab, horseshoe crab, eastern oyster, freshwater mussels, and other macroinvertebrates (https://www.state.nj.us/drbc/library/ documents/SOTBreport_july2019.pdf). Understanding the response of living resources and their habitats to management actions and ecosystem change is the next stage in the scientific priorities identified by partners for the Chesapeake and Delaware watersheds. The goal of this project is to create an USGS interdisciplinary science team focused on conducting integrated science to inform the management of recreational and commercially important fish species, waterbirds, benthic macroinvertebrates, other aquatic and semi-aquatic species, pollinators, and their habitats. This team would establish an integrated research framework similar to Chesapeake Bay to provide a multidisciplinary approach to the management of the Delaware River Basin. Essentially what are the drivers of the ecosystem, how do the stressors impact those drivers, what are the cumulative impacts of these drivers and stressors on the habitat quality and availability on the fish, wildlife, and people that use the watershed. Ultimately, providing recommendations to the Delaware River Basin Restoration Program and other stakeholders on the conservation, management, and restoration of these resources/habitats to sustain the living resources in the ecosystem. In addition, identifying gaps in our knowledge that would be needed to better inform the management actions for living resources. Ultimately, with similar research efforts being conducted in both watersheds (Chesapeake and Delaware), management decisions and restoration recommendations could be made on a broader regional scale.

Stakeholders are essential to this project and examples of local, state and federal stakeholders include, but are not limited to, Delaware River Basin Commission, Partnership for the Delaware Estuary (PDE), Delaware Department of Natural Resources and Environmental Control (DNREC), Department of Fish and Wildlife (DE DFW), United States Fish and Wildlife Service (USFWS), Delaware Water Resources Center, Institute for Public Administration, University of Delaware, USGS New Jersey [NJ] Water Science Center, Maryland-Delaware-D.C. [MD-DE-DC] Water Science Center, Lower Mississippi-Gulf [Lower MS Gulf] Water Science Center, and Woods Hole Coastal Marine Science Center, Atlantic States Marine Fisheries Commission, Migratory Bird Initiative and Migratory Connectivity Project: Smithsonian Migratory Bird Center, Georgetown University, National Audubon Society, and Cornell Lab of Ornithology. Many of these partners are already actively collaborating on research projects for the Chesapeake Bay. Table 5.1 highlights the alignment of the Delaware Basin Plan goals, with USGS priorities and Mission Areas, DOI Priorities, and the Chesapeake Watershed Agreement goals. 
Table 5.1. The alignment of the Delaware Basin Plan goals with U.S. Geological Survey (USGS) priorities and Mission Areas, Department of the Interior priorities, and the Chesapeake Watershed Agreement goals (adapted from USGS CB Science Plan 2020-25; Hyer and Phillips, 2021).

[USGS, U.S. Geological Survey; DOI, Department of the Interior]

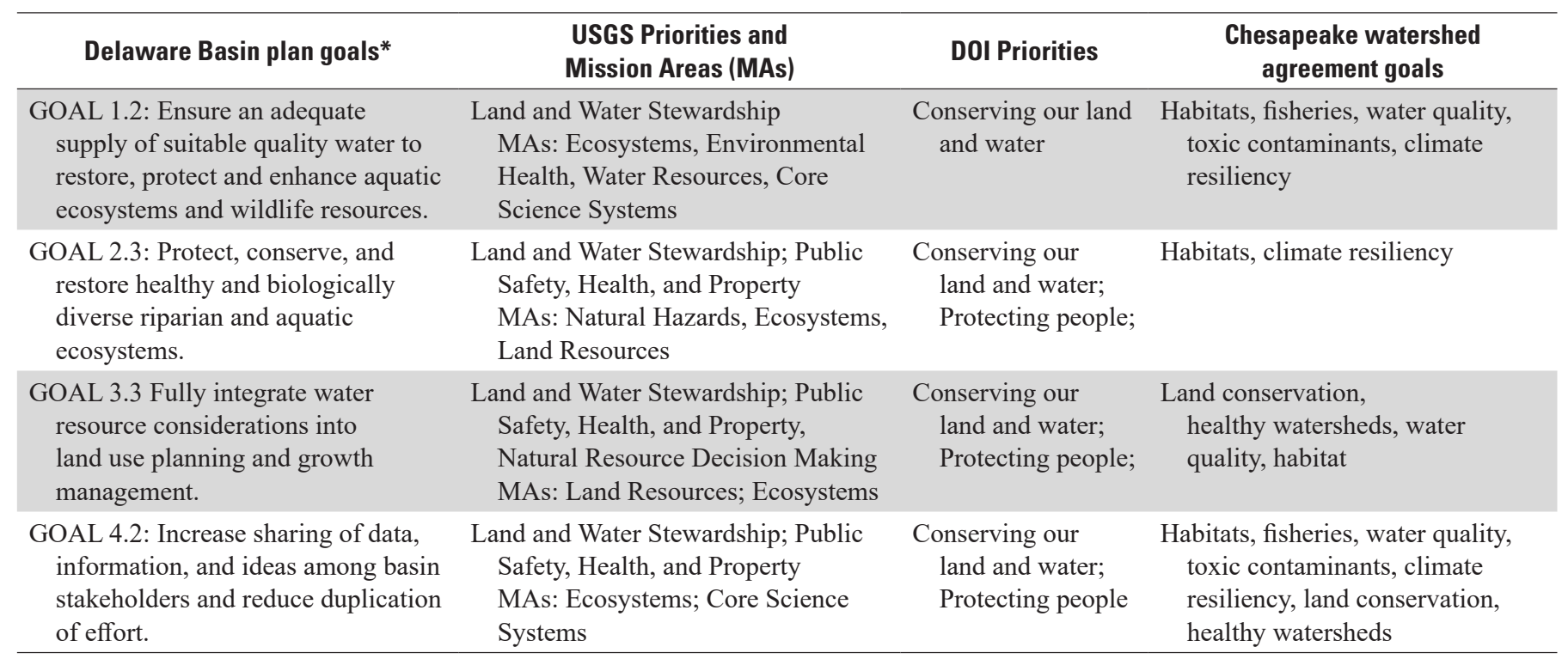

*https://www.state.nj.us/drbc/library/documents/BasinPlan_Sept04.pdf.

\section{Improved Decision-Support}

One activity identified by the Delaware River Basin Restoration Program is to increase scientific capacity to support planning, monitoring, and research activities necessary to carry out coordinated restoration and conservation activities in the basin (https://www.fws.gov/northeast/delawareriver/ partnership/index.html). The goal of this Use Case activity is to increase coordination of scientific resources among partners in the basin, supporting project planning, decision making, and measuring improvements to natural resources after project implementation, mirroring ongoing efforts in the Chesapeake Bay. Over the last decade, PWRC/LSC researchers have made considerable progress implementing interdisciplinary integrated science in the Chesapeake Bay. With the additional research capabilities of this USGS science team, we could provide cutting-edge modeling efforts and decision support tools to the Delaware River Basin Restoration Program and other key stakeholders. For example, using structured decision making to create spatial value models and visualizations to identify important landscapes for conservation and (or) optimizing salt marsh management.

\section{Science Capabilities and Advancement Opportunities}

PWRC/LSC researchers have been working in the Delaware River Basin for many years now and have compiled quite an extensive multidisciplinary dataset on, but not limited to, amphibian distribution, fish community ecology, habitat suitability, decision support tools, listed species ecology and management, fish health data, terrestrial/ freshwater organismal records, harmful algal bloom data, avian population abundance and distribution data, influences of river flow and temperature on fish communities, freshwater mussel data, pollinator distributions, avian disease data and linkages to poultry industry, avian health data, invasive species, and water contaminant data. Much of this research is in collaboration with other science centers through the USGS Chesapeake Bay Science team (USGS NJ Water Science Center, MD-DE-DC Water Science Center, Lower MS Gulf Water Science Center, and Woods Hole Coastal Marine Science Center). New approaches are needed for this project to adapt the Chesapeake Bay integrated modeling framework for the DRB. For example, the NHD-HR Hydrolink proposed Use Case will develop the capability to link biological data to the medium resolution National Hydrography Dataset Plus (NHDPlus, 1:100K) and newly developed National Hydrography Dataset Plus High Resolution (NHDPlus HR, 1:24K). This step is necessary in order to summarize landscape variables in catchments surrounding stream reaches for aquatic habitat assessment and align with efforts to build out the National Hydrography Infrastructure (NHI) to provide information at the highest resolution, decision-relevant scales that stakeholders have requested. 
Operational Implementation and Technical Considerations

There are two potential avenues of implementation to achieve this project goal depending on the resources available: 1) Leverage off the existing PWRC/LSC USGS Chesapeake Bay research activities that could be adapted or expanded into the Delaware River Basin with minimal additional resources or 2) develop a new USGS Delaware River Basin Science Team. This USGS Science Team could maintain the data storage, access and delivery of datasets and products to internal and external partners via a project site that could be linked to the Delaware River Basin Restoration Program website to encourage stakeholder engagement.

\section{Longevity and Sustainability}

Managers and decision-makers will continue to need scientific data and modeling to make informed decisions for managing human and natural communities in an ever-changing climate. The USGS Delaware River Basin Science Team would continually evolve, similarly to the Chesapeake Bay Science Team, to ensure USGS fulfills the needs of the stakeholders as they adapt to better manage the system. For instance, the structured decision-making models can evolve as the decisions needed change and the associated framework is scalable so the resolution of the output can change based on the scale of the input data.

Primary Contact: Tom O’Connell, toconnell@usgs.gov; Alicia M. Berlin, aberlin@usgs.gov

\section{Improving National Fish Habitat Assessments Using the NHDPlus HR: An EarthMAP Pilot Effort in the Delaware River Basin}

Natural resource managers and decision-makers have consistently identified the critical need for information at finer spatial scales (for example, 1:24,000) in order to more effectively design and implement habitat assessments and associated conservation, mitigation, and restoration activities and better inform decisions. This project will build on current interdisciplinary science efforts to enrich geospatial datasets and to provide the basis for enhanced information discovery and management relevant data delivery to stakeholders faster via EarthMAP.

\section{Demand and Relevance}

The National Fish Habitat Partnership (NFHP) is a national effort that brings together partner organizations from Federal and State agencies and NGOs with the purpose of identifying, enhancing, and restoring fish habitats. USGS is an active partner in NFHP with leadership roles served by Science, Analytics and Synthesis (SAS) and Ecosystem Mission Area (EMA). A major project of this partnership was an assessment of the status of fish habitats in the US, last conducted in 2015, using nationally consistent environmental data and fish community observations from partnering agencies. While an incredible resource for national and regional comparisons, this assessment was necessarily limited to data available nationwide, and was limited to the regional-scale $(1: 100,000)$. However, stakeholders and natural resource managers have consistently identified the critical need for information at finer spatial scales (for example, 1:24,000) in order to more effectively design and implement habitat assessments and associated conservation, mitigation, and restoration activities and better inform decisions.

However, current efforts to integrate high resolution data (for example, the National Hydrography Dataset NHDPlus High Resolution [NHDPlus HR] Beta version at 1:24,000) into decision making have revealed issues and limitations of existing information that must be addressed before these efforts can move forward. These include resolving issues with catchment boundary delineations which hinder summarizing landscape stressors associated with stream reaches and connecting to existing biological data. For example, current data collected by partner agencies from 1:24K mapped stream features have no equivalent representation at the coarser spatial scale losing up to 10 percent of fish observations that cannot be used resulting in loss of data integrity, increased uncertainty, and limiting decision relevance.

A pilot project that includes scientists with Core Science Systems, Ecosystem Mission Area, and USGS Water Resources Mission Area is addressing the technical challenges connecting NHDPlus HR data to biological data to improve modeling landscape-aquatic habitat interactions at management relevant scales in the Chesapeake Bay Watershed (mapped in USGS NHD HydroRegion 02). Correcting issues with the NHDPlus HR will allow for more effective and accurate monitoring, assessment, and prediction — key elements of the EarthMAP vision.

This Use Case will expand this effort to align geospatial and biological data for fish habitat assessments in additional river systems in NHD HydroRegion 02, particularly the Delaware River Basin (DRB), which has been designated a national treasure of great cultural, environmental, ecological, and economic importance. The capability to link biological data to the medium resolution National Hydrography Dataset Plus (NHDPlus, 1:100K) and newly developed National Hydrography Dataset Plus High Resolution (NHDPlus HR, $1: 24 \mathrm{~K}$ ) is a necessary step in order to summarize landscape variables in catchments surrounding stream reaches for aquatic habitat assessment. and align with efforts to build out the National Hydrography Infrastructure (NHI). Achieving this capability will help USGS provide information at the highest resolution, decision-relevant scales that stakeholders have requested. 
The DRB contains 23,700 linear miles of streams and rivers and expansive groundwater systems supporting nearly 180 species of fish and wildlife that are considered special status species in the Basin due to habitat loss and degradation, particularly sturgeon, Eastern oyster, horseshoe crabs, and Red Knots. In addition, the DRB provides habitat for over 200 resident and migrant fish species, includes significant recreational and commercial fisheries including Eastern brook trout, American and hickory shad, river herring, and American eel. Fishery managers are no longer able to sustain our nations fish, fisheries and aquatic habitats solely by regulating harvest, but need to better understand how the connections between landscape changes and aquatic habitats affect ecologically and socio-economical important fish and aquatic resources. USGS EMA is currently conducting habitat, population, and stock assessment research on several of these species in support of partners including the Atlantic States Marine Fisheries Commission (ASMFC) and NPS.

\section{Improved Decision-Support}

The approximately 135 major stakeholders in the DRB have identified a suite of conservation and restoration needs that require scientific information at fine spatial scales in order to achieve their goals and outcomes. These include:

- Delaware River Basin Restoration Act

- USFWS-Delaware River Basin Restoration Partnership Program Framework

- USFWS-National Listing 7-yr Workplan for at-risk species

- USFWS-ESA recovery plans and habitat designations

- NOAA-Recovery plans, status reviews

- ASMFC - Interstate Fishery Management Plans, Stock assessment reports

- DRBC-Delaware River Basin Compact, DRBC Comprehensive Plan, Flexible

\section{Flow Management Program}

- NPS - Wild and Scenic Rivers Act, DE River Basin Wild and Scenic River

- Values Workshop Report

The goals of the Delaware River Basin Restoration Partnership Program Framework include 1) sustaining and enhancing fish and wildlife habitat restoration and conservation activities and 2) increasing coordination of scientific resources among partners in the basin to support decision making for project planning and measure improvements to natural resources after project implementation. Their Focus Areas for Conservation Prioritization and Design include:

- Improved inventory and monitoring of existing waterways, land use, and ecological conditions to determine priority areas for project implementation

- Provide monitoring capabilities to assess improvements to natural resources based on implementation of Program projects

- Establish science-based decision support tools to determine priority areas for conservation and restoration projects

- Evaluate effectiveness of projects to increase citizen's stewardship of natural resources

- Document economic benefits of project implementation

- Establish data management and data analysis capabilities, as well as provide a mechanism for data sharing between Program partners to support Program projects and priorities.

Results of the this Use Case project will support those conservation and restoration goals and priorities. This methodology, including alignment of data to the National Hydrography Infrastructure, can serve as the framework for national efforts to enrich geospatial datasets and to provide the basis for enhanced information discovery and management relevant data delivery to USGS stakeholders, such as through the EarthMAP platform.

\section{Science Capabilities and Advancement Opportunities}

The USGS Core Science Systems Mission Area National Geospatial Program (NGP) is developing the National Hydrography Infrastructure (NHI) to support the referencing and discovery of water-related information, which in turn, will help support hydrography-based modeling efforts. The hydrographic framework for the NHI is the NHDPlus HR, which represents stream networks at 1:24K scale and provides intelligent stream flow routing and landscape attributes similar to the highly successful medium resolution NHDPlus used in numerous science and assessment activities, including the NFHP. Currently in a Beta release, the NHDPlus HR will serve as a critical framework for integrating a wide variety of USGS and partner agencies' observational information to fine-scale stream reaches as well as for summarizing environmental factor data in local and upstream drainage areas and catchments. 
The Delaware River watershed is being used as a pilot for USGS integrated science activities, including WMA's Integrated Water Prediction and Next Generation Water Observing Systems implementation. Key to translating these efforts to National implementation are framework geospatial datasets, including the NHD Plus HR. In addition to USGS WMA data on water quality and quantity, USGS EMA (Leetown Science Center) scientists have previously conducted extensive eastern brook trout, freshwater mussel, stream temperature, stream habitat, and submerged aquatic vegetation studies in the basin. Additionally, they have developed bathymetric and topographic LiDAR datasets for the mainstem Delaware River that can provide the basis for 2D hydrodynamic modeling of river flow velocity. These data can be used to further enrich the NHD HR framework to add value to integrated modeling activities. The stakeholders and partners noted under 2 have extensive biological and ecological data that is available for this effort. This Use Case will leverage all of these existing data sources.

\section{Operational Implementation and Technical Considerations}

This Use Case will build on the existing multidisciplinary, integrated Chesapeake Bay science team to expand efforts in the DRB. The Chesapeake Bay Program already supports extensive data frameworks to store and deliver datasets and products to internal and external partners. USGS is already working with most of the major stakeholders identified under 2 and will further those collaborations as part of this project.

\section{Longevity and Sustainability}

Developing the capabilities to update the NHDPlus HR data such that it is useable for modeling landscape-aquatic habitat interactions at management relevant scales will support more effective and accurate monitoring, assessment, and prediction. It is anticipated that the methodologies developed in this project will be transferable to other areas nationwide.

Primary Contact: Tom O’Connell, toconnell@usgs.gov; John Young, jyoung@usgs.gov

\section{Optimization of Mineral Resource Data: Mine Wastes, Resource Assessments, and Focus Areas}

The proposed Use Case presented here leverages existing, ongoing, and planned science products to fulfill the needs of internal and external stakeholders with regard to mineral resources, including assessments for potentially economic resource extraction, remediation and recovery of valuable products from mine waste material, and priority areas for data acquisition.

Ongoing work in the USGS Mineral Resources Program represents:

\section{Demand and Relevance}

Population growth and increased standards of living have accelerated societal demand for mineral resources. Technological advances and the clean energy transformation will require increased quantities of minerals critical for their function. Decisions affecting the future supply of mineral resources must balance sustainability, environmental impacts, national security, and economic factors. The overarching question that this Use Case addresses is: where should mineral resources be extracted to 1) maximize economic efficiency, sustainability, and security of supply and 2) minimize hazards to human health and the environment and risks of supply disruption?

Stakeholders include State Geological Surveys, elected officials in Congress and State legislatures, decision makers at the Federal to local level, including such agencies as the Bureau of Land Management (BLM), the U.S. Forest Service (USFS), National Park Service (NPS), Department of Energy, Department of Defense, Department of Commerce, and the Environmental Protection Agency. Additionally, the information should be made publicly available for use by industry, nongovernmental organizations such as the World Bank, international organizations such as the United Nations International Resource Panel, and academic researchers. Furthermore, access to mineral resource information contributes to the Department of Interior goals of stewardship of natural resources and conservation.

Relevant stakeholder questions include: Where should mineral exploration activity be focused? What areas should be off-limits to mineral activity? Where do mine wastes pose risks to the environment and human health? Where in the United States could a specific mineral commodity be economically mined? As some of these questions require evaluating competing land uses, mineral resource information should be presented alongside other data in EarthMAP such as protected areas, biodiversity hotspots, environmentally sensitive areas, water resources, and agriculturally important areas. 
Geospatial information on where minerals have been mined in the past, the location of wastes associated with their production and processing, and prospective areas for future mineral production is crucial for resource policy decisions, economic planning, land use management, and environmental regulation. The USGS Mineral Resources Program provides data, expertise, and analysis necessary for addressing this question. In the past, regulatory and land management agencies have sought input and information directly from the USGS to inform land planning decision making, as required by the Federal policy. As a result, the USGS has produced a body of work contained in publications, maps, and data releases over many decades. While the scale and scope of each science varies depending on the goals of the project, much of this information remains relevant and useful for guiding future work.

\section{Improved Decision-Support}

Stakeholders weigh the economic value provided by mineral resources against the risks and costs associated with mineral extraction. To make decisions, they must clearly understand the uncertainties associated with each layer of information.

The Federal Land Policy and Management Act (FLPMA) requires land management agencies (BLM, NPS, USFS) to consider mineral resources in their long-term land use planning. Past assessment work has focused on specific areas, such as Wilderness Study Areas, proposed for withdrawal and been published at a relevant scale on an as-needed basis. A specific example is the Sagebrush Mineral-Resource Assessment (SAMiRA) assessment of mineral resource potential of Greater Sage-Grouse habitat on BLM lands. This assessment delineated where disturbance through mineral extraction of a critical habitat could pose further stress to a threatened species. This assessment provides the fundamental scientific information to enable BLM to make wise decisions regarding land use in Greater Sage-Grouse habitat. Leveraging this published data by making assessment results more accessible to stakeholders will facilitate prioritization of future assessments by identification of crucial focus areas that need to be assessed.

Other stakeholders, such as State Geological Surveys, currently work with the USGS to determine focus areas for geological investigations funded under EarthMRI (Hammarstrom and others, 2020). These focus areas are selected because they encompass geologic deposits that have significant potential for important mineral occurrences. Identification of these focus areas will help prioritize where research funding should be allocated to maximize the ability to make informed decisions regarding mineral resource extraction and its impact on the environment and human health.

\section{Science Capabilities and Advancement Opportunities}

Existing foundational data have been published by the USGS in a variety of formats and are currently hosted by several different platforms. MRDATA (Mineral Resources Online Spatial Data) contains much of the spatial data in a form amenable to EarthMap. Permissive tracts from previous mineral resource assessments exist as maps in the USGS publications warehouse and National Geologic Map Database (NGMDB) or GIS files in ScienceBase. Active mines and abandoned mines may be spatially associated with mineral sites present in the Mineral Resources Data System (MRDS) and USMIN mineral deposit database (USMIN) databases, accessible through MRDATA and ScienceBase. Externally, information on abandoned mine lands is maintained by the Office of Surface Mining, Reclamation, and Enforcement (OSMRE), the EPA, BLM, and an interagency effort (https://www.abandonedmines.gov/) that includes the USGS.

There is limited information technology (IT) support to facilitate integrating science products hosted in Science Base and other repositories into EarthMap. Currently, science products are published and disseminated through USGS publications warehouse, ScienceBase, and in the case of mineral resource assessment maps, the National Geologic Map Database (NGMDB). The simplest starting point for integrating this information into a spatial platform is to link shapefiles of study areas to their original publications. A next step would require the digitization of legacy datasets and reattribution of digital polygon files for permissive tracts. This work has been carried out for a few study areas, but not on a national scale. Work initiated through the National Geological and Geophysical Data Preservation Program has resulted in the curation and preservation of data in dozens of States and has forged partnerships with State Geological Surveys.

New approaches to disseminating assessment results may need to be developed to address decision-maker and stakeholder expectations. Past assessment results are a mix of quantitative and qualitative results depending on the project or client. Application of economic filters in resource assessments will provide new data that will be of interest to a variety of potential partners in the mining sector.

Mineral Resource Assessment constitutes a core function of the USGS Mineral Resources Program. Expertise in this area has enabled the training of several early- and mid-career scientists with the capacity to carry out assessment work and contribute toward new results. 
Operational Implementation and Technical Considerations

There is limited existing operational support for the Use Case. In the past, mineral resource assessment work has been funded by appropriations from Congress and reimbursable funding provided by partner agencies. The foundational-level input data utilized to conduct mineral resource assessments exists.

In the process of integrating multiple data sources supporting mineral resources assessment work, it would be critical to define whether the data in use are readily available in terms of their level of processing, relative quality, storage formats (as is the case of remote sensing, for example), and whether it can be easily brought up to satisfy the user needs. For example, the Mineral Resource Data System (MRDS) was recently updated with a "grade" of record qualities where a category of record completeness for each mineral deposit was estimated (https://www.sciencebase.gov/catalog/item/5d71 $2 \mathrm{a} 5 \mathrm{ae} 4 \mathrm{~b} 0 \mathrm{c} 4 \mathrm{f} 70 \mathrm{cfd} 4 \mathrm{e} 54)$. This information allows users to better query data based on quality to ensure the data meet their project needs. Partnerships are being developed with State Geological Surveys through Earth MRI to incorporate more and better-quality data into MRDS. Similar efforts to carefully assess and present information on record quality will be necessary to increase the usability of the integrated mapping platform. Furthermore, the user should be able to identify and access the technical or scientific support necessary to improve on the existing datasets, such as specialized software licensees who can develop and deliver specific products. An example of this is the development of mineral alteration maps from remote sensing data. Such maps can be useful for recognizing particular mineral deposits, which would draw in partners from the mining sector, or can pinpoint potential natural hazards associated with volcanoes, which would benefit emergency management and response agencies, at both the national and State level.

\section{Longevity and Sustainability}

The U.S. Geological Survey has, since its inception, provided expertise in the area of mineral resources of the United States and will continue to meet the needs of Federal government agencies, fulfilling its original mission defined by the Organic Act of 1879. A tiered approach to implementing the mineral resource component of EarthMAP is envisioned. At a minimum, the existing publications and geospatial data currently hosted on USGS platforms (Science Base, MRDATA, NGMDB, and Publications Warehouse) could be linked to or added to the EarthMAP infrastructure. The next phase of implementation may require digital conversion of legacy dataset, for example, the type of work that was conducted for the SAMiRA project.

The implementation of a mineral resources Use Case can exist at virtually any scale. Typically, assessments have been conducted at 1:100,000 to $1: 1,000,000$ scales. The presentation of this level of detail at a national scale is envisioned.

A smaller-scale Use Case, for example, at the state or regional level, may represent a good starting point for implementation. Eventually mineral resource capability of EarthMap could be scaled-up to a national level. Successful implementation can be measured soliciting stakeholder feedback to gauge ease of use while maintaining an adequate level of complexity needed to address policy and regulatory missions.

Primary Contact: Graham Lederer, glederer@usgs.gov

\section{Collaborative Science to Enhance Resilience in Coastal Cities, Towns, and Rural Landscapes: The Urban Coastal Resilience Initiative (UCRI)}

The challenge of maintaining the functions and livability of the Nation's coastal cities has focused attention on building resilience in developed and adjacent natural coastal landscapes. Hazards to coastal inhabitants and commerce are increasing due to continued expansion and aging of infrastructure, sea-level rise (SLR), loss of natural buffers, and storm surge. A whole-system approach to science is needed to effectively inform management and policy decisions for urbanized, natural, rural, and fragmented coastal landscapes.

\section{Demand and Relevance}

The goal of this Use Case is to provide science information for enhancing the resilience of natural and built environments facing significant and increasing changes in current baseline conditions and disturbance events. Predictive science on resilience of coastal regions will inform adaptations to greatly reduce the costs in lives, property, and infrastructure of anticipated chronic and catastrophic disturbances.

The NAAR Urban/Coastal Resilience Initiative (UCRI) will integrate goals from all the current USGS Mission Areas (MA), the 4 Water Centers, several programs including the Coastal and Marine Hazards Program, the Science and Decisions Center, Core Science data and mapping programs, and several Ecosystems MA Programs. Collaborations with the Southeast and Great Lakes Regions are likely as the Initiative advances. Interested outside partners include the New York City (NYC) Mayor's Office of Resiliency (MOR) and Department of Environmental Protection (DEP), Parks \& Recreation (Parks), and Transportation (NYDOT); NOAA; FEMA; USACE; USFWS; and The Nature Conservancy (TNC). 
Multiple issues will be addressed by the UCRI using a meta-project, multi-scale, interdisciplinary approach that reflects the EarthMAP goals, but for this Use Case we will describe one example: development of coupled models of compound flooding and its effects on developed and natural coastal landscapes. Natural resource and infrastructure managers and policy makers will be the primary stakeholders in this Use Case.

Specific stakeholder interest in this Use Case:

1. Example Decisions this EarthMAP Use Case will inform:

What NYC Boroughs need adaptation to avoid compound flood damage? Where do we need to evacuate for storms, and where do we need to retreat? Where could wetlands, living shorelines, or natural or nature-based infrastructure be established to protect or enhance valued resources?

\section{Relevant decision-makers and their need for improved} science information.

a. Municipal managers and planners: Best practice solutions for specific conditions

b. NGO environmental and health organizations: flood and erosion vulnerability

c. Flood- or erosion-prone communities: hazard frequency, severity and escape routes

d. Transportation, health and human service managers: what/when to prepare/protect

\section{Integrated science and information synthesis necessary:}

Coupled models of the key processes controlling resiliency based on whole-system responses to directional change and episodic disturbance (groundwater, surface water, SLR, and storm surge; and the infrastructure, ecological, and socio-economic effects). Coupled-Ocean-Atmospher e-Wave-Sediment Transport Modeling System (COAWST), Hydrologic Engineering Center's River Analysis System (HEC-RAS), and MODFLOW will be coupled to allow groundwater dynamics to be influenced by surface water and inundation from coastal waters to be influenced by groundwater processes. Socio-economic and ecological models will be linked to the water models and applied to infrastructure maps to map resources and communities at risk.

\section{Strategic real-time monitoring, rapid assessment, and} early warning:

Groundwater monitoring arrays will be linked to the Surge, Wave, and Tide Hydrodynamics (SWaTH) network, tidal wetland SET stations, tide monitoring (NOAA and
USGS) and surface water gaging, creating an array of continuous fixed stations, rapid deployment arrays, periodic surveys, and remote sensing for whole-system, multi-scale (time and space) tracking.

\section{Relationship to known priorities:}

City planners have a pressing need for compound-flood modeling capabilities, particularly as they relate to coastal adaptation, stormwater infrastructure, and transportation planning. The limited existing models for cities are not yet available to smaller coastal towns.

\section{Extended science and products at different spatial scales and time periods.}

This integrated modeling framework could be deployed to evaluate risks over a planning time horizon and to account for issues that will occur with SLR and changing climate. It could also be deployed in a more operational framework to understand short term risk of disturbance.

\section{Improved Decision-Support}

As environmental disturbance events increase in number and magnitude, and human populations grow in coastal cities and towns, urban infrastructure undergoes multiple stressors and, in many instances, the damaging impacts are further exacerbated by the mere fact that many are aging and decaying already. Additionally, with increased environmental disturbances and human population growth, natural landscapes are continuously being degraded and wildlife populations in or near urban areas are being displaced. Developing coherent, longer-term, location-specific resiliency for urban areas requires a science strategy that produces interdisciplinary and multi-scale information from all available sources. The USGS, with its multi-scale, interdisciplinary capabilities in data collection, management, and synthesis, will use this Urban/ Coastal Resilience Initiative (UCRI) to provide the integrative leadership needed for delivering regionally-focused, systems-level environmental science for timely informing of management and policy decisions. Specific goals for improved decision support include anticipation of hazards and best practice recommendations for mitigation or managed retreat, early warning, and enhanced accuracy, availability, timeliness, and utility of USGS monitoring and interpretation. Close collaboration with stakeholders will establish these milestones. The improved modeling will allow robust assessments of coastal adaptation pathways for managed retreat, better anticipation of evacuation requirements, and improved planning for infrastructure operation, maintenance, and replacement. 


\section{Science Capabilities and Advancement Opportunities}

The UCRI will applied state-of-the-art modeling and monitoring network design (see Delaware NGWOS) for each issue addressed. Machine learning models, artificial intelligence, and remotely sensed data collection will be used where they afford better or more cost-effective and efficient assessment of the processes and conditions affecting resource decisions. Hydrologic models will be linked with socio-economic maps and models, ecosystem vulnerability assessments, and natural, nature-based, or grey infrastructure disturbance mitigation strategies for assessments of whole-system responses to change. Collaborations with stakeholders throughout the model development will guide products toward supporting resource and infrastructure management and planning decisions.

Existing capabilities in the Northeast are extensive both within USGS and our Federal, State, municipal, academic, and NGO partners. Monitoring records are available for mapping, modeling, and trend detection. Examples include: NYCDEP water monitoring, NYWSC observation wells, streamgages, tide gages, and water-quality networks; and the SWaTH rapid deployment network. State-of-the-art models for the coupled model strategy include COAWST, SHERA, CoSMoS, and the Long Island Regional groundwater and solute-transport models. Established collaborations with coastal modeling efforts by other agencies and academia will be enable further integration of modeling capabilities and potential products for national transferability (NOAA Coastal Coupling Community of Practice (CCCoP), National Science Foundation Coasts and People Program (CoPe). The initiative has access to significant computational and data management capabilities in the Water and Coastal and Marine science centers of the NAAR to initiate the modeling. The goal over time is to fully integrate the IT requirements for UCRI into the services being designed by the national EarthMAP initiative.

The Use Case will develop several new capabilities and approaches, incorporating new technology and better integrated strategies for applying existing technology when it results in more efficient or robust measurement and interpretation. This initiative will rely extensively on the leveraging of existing projects and capabilities within the NAAR. Potential for new funding from stakeholders is significant, but the issues being addressed will require a funding strategy of leveraging existing funds with supplemental financing to be cost effective. This strategy also leverages data collected by prior investigations, decreasing the time required to detect trends and accelerating delivery of actionable intelligence. Multiple existing datasets are available for many issues the UCRI will address, through
USGS archives and partner databases. For example, detailed below-ground mapping of water infrastructure in New York City compiled by NYCDEP will allow a significant improvement in groundwater modeling to determine infiltration rates and saturation potential affecting compound flooding. Partnerships will depend on the issue being addressed, but the potential is significant because of a long history of successful USGS collaborations in the region. Partnerships for the compound-flood modeling in NYC include NYC MOR, DEP, Parks, and NYDOT; FEMA, NOAA, NYSDEC, and several academic institutions.

\section{Operational Implementation and Technical Considerations}

1. Current and potential technical capacity: Technical capacity for the compound flooding initiative is available within USGS and with established partners. A Letter of Intent to initiative the modeling work in NYC has already been formalized by the partners.

2. Current and potential approaches for science delivery: The NYC DEP and MOR are the initial partners in the compound flooding proposal. They will collaborate with USGS to ensure modeling products are immediately useful to decision- and policy-making. A parallel co-production strategy will be applied to each UCRI project.

3. Data storage, legacy data access, data lineage, and data-delivery mechanisms: The initiative has access to significant computational and data-management capabilities in the Water and Coastal and Marine science centers of the NAAR to initiate the modeling. The goal over time is to fully integrate the IT requirements for UCRI into the services being designed by the national EarthMAP initiative.

\section{Potential science and technology partners: (Already} named above).

5. Additional resource needs: Letters of intent for two projects for NOAA funding have been submitted by USGS and partners. NYWSC may contribute matching funds to City funding to build longer-term collaborations. Further funding will be needed as other projects in the UCRI evolve. Services-in-kind agreements with Stevens Institute will provide subsurface infrastructure expertise, and with NYCDEP will yield infrastructure and surface water-quality information needed for the modeling. 


\section{Longevity and Sustainability}

1. Anticipated long-term relevance of this Use Case: Compound flooding is an issue in many coastal cities and towns and is likely to remain so for the foreseeable future. Providing better planning capabilities and early warning of hazards will result in significant reductions of loss of life, health, infrastructure, natural resources, and commerce.

2. Requirements to sustain this effort: Success with this modeling effort should pay for itself as other municipalities become aware of and apply its capabilities. Staff, IT, and monitoring are legacy requirements.

3. Transferability and scalability of deliverables: The compound flood models will be transferable and scale-able throughout the U.S. coastal zone and beyond. Once the coupled modeling framework is developed, the physical processes that link groundwater and coastal water can be described in any location with the normal model development process.

4. Evaluating the effectiveness of this effort and defining performance metrics: Prior work by USGS and partners on defining physical, ecological, and socio-economic metrics of success for Hurricane Sandy is available for assessing the UCRI success.

5. Benefits at different scales, timeframes, or geographies: Applications to other coastal cities and regions will improve planning, early warning, and cost-effective management of coastal infrastructure and resources. The UCRI is multi-scale (for time and space) to enable projection from where data is gathered to where decisions are made. The models address geographic and geophysical conditions and so are readily applicable to other landscapes.

Primary Contacts: Peter S Murdoch, pmurdoch@usgs.gov; Chris Schubert, schubert@usgs.gov; Liv Herdman, lherdman@usgs.gov

\section{Northwest—Pacific Islands}

\section{Thunder and Lightning-Social, Ecological, and Hydrologic Assessment of Drought in the Donner und Blitzen River Watershed}

This Use Case is uniquely positioned to provide a working example of how products from EarthMAP can be developed and applied to respond to immediate and anticipatory management decisions to address the increasing threat posed by drought on social, ecological, and hydrological systems. The Donner und Blitzen River represents a Use Case for EarthMAP that offers strongly leveraged data, stakeholder readiness, and alignment with the USGS Integrated Drought Science Plan.

\section{Demand and Relevance}

Drought represents one of society's grand challenges for the 21st century (Crausbay and others 2020). Impacts of drought are especially evident in the western United States, where precipitation deficits increasingly coincide with warmer temperatures, impacting human and ecological water availability. Managers seeking to address drought include those responsible for managing millions of acres of federal public lands in the western United States administered by the U.S. Forest Service, Bureau of Land Management, and National Park Service. These lands are critical for providing innumerable societal benefits and managers of them are in urgent need of reliable, accessible scientific information to inform difficult decisions ranging from water security to ecosystem management. To address these needs, the USGS has developed a coordinated and integrated drought science plan to address the issues of drought and build long-term resilience to drought at the local, regional, and national levels (Ostroff and others, 2017). Eco-Drought is the on-the-ground implementation of the USGS Integrated Drought Science Plan. Implementation of Eco-Drought has involved shared resources and collaboration across three USGS Mission Areas, including Water Resources, Ecosystems, and Core Science Systems. Work in the Use Case described herein is also part of 5 major new investments in the NOAA-NIDIS "Coping with Drought" program (National Oceanic and Atmospheric Administration). 


\section{Improved Decision Support}

Products from the USGS Eco-Drought effort are intended to address drought at scales and time periods that are salient for managers who face difficult on-the-ground decisions. Drought can manifest quickly (for example, "flash droughts") or take place over much longer time frames to act more as a press disturbance that potentially leads to a fundamental ecological and potentially social transformation (Crausbay and others, 2020). Similarly, the spatial grain at which drought responses are considered can influence perceptions of impacts and appropriate management responses. Kovach and others (2019) provided the example of how USGS streamgages are often mis-aligned with ecological observations in drought-sensitive river networks. Addressing this alignment to decision makers needs is one of the central themes of the USGS Eco-Drought studies. In most of these studies, stakeholders have been directly engaged in providing feedback on relevant questions and often providing funds to leverage USGS investments. Tools that allow them to easily collect complementary information for tracking drought on the ground have also been developed (FLOwPERmanence [FLOwPER]; Jaeger and others, 2020). In short, stakeholders have been engaged to inform research direction and objectives, as well as to collect data in many cases. Regardless of the specific decision or resource addressed, this level of engagement is certain to ensure actionable outcomes (Dunham and others, 2018).

\section{Science Capabilities and Advancement Opportunities}

Existing scientific and technological capacity. Existing scientific and technological capacity is strong among the collaborators working in the Donner und Blitzen study, but additional capacity would likely be needed to support additional requirements of EarthMAP. Expertise on the scientific team presently engaged in this work includes aquatic and landscape ecology, remote sensing, Bayesian statistical modeling, hydrologic modeling, and modeling of soil moisture. Additional time and expertise to adapt emerging modeling platforms for prediction of hydrologic responses (for example, Read and others 2019), data processing and visualization, and time for stakeholder engagement (Delie and Biedenweg, 2019; Miller and others, 2020) is needed to ensure this effort is a success from data to decisions.
Potential for integration and existing datasets. This study includes most of the elements of other USGS projects, such as the Integrated Water Availability Assessments recently developed (Miller and others, 2020). Existing USGS and partner datasets are being assembled now for integration in the Eco-Drought and related studies ongoing in the Donner und Blitzen basin and could be integrated into EarthMAP. Partnerships are strong in the basin and would be significantly strengthened locally and regionally through this effort. In addition to the Eco-Drought work in the Donner und Blitzen, USGS is actively engaged in a long-term study of groundwater use and availability and persistence of springs within the broader extent of the Harney Basin (Steve Gingerich and Hank Johnson, personal communications, U.S. Geological Survey Oregon Water Science Center [ORWSC]). A recent study involving USGS (Forest and Rangeland Ecosystem Science Center [FRESC] and ORWSC) and USFWS (Pearson 2020) also provides information on flow-dependent persistence and restoration of wetlands in the Malheur National Wildlife Refuge that could be well-informed by implementation of EarthMAP. Finally, non-market economic valuation led by USGS (Lucas Bair, U.S. Geological Survey Southwest Biological Sciences Center, personal communication) could be integrated into this work, providing a full complement of tools for evaluating this case as a social-ecological system (Dunham and others, 2018).

Existing and potential partnerships. The Donner und Blitzen River watershed is part of the larger internally draining Harney Basin, located in southeastern Oregon, USA. This watershed is a cooperative management area administered by BLM and includes Wilderness and Wilderness Study areas, as well as designated multiple use lands. The river itself is a designated Wild and Scenic River and Redband Trout Preserve. The river provides critical flows that sustain wetlands downstream in the USFWS Malheur National Wildlife Refuge (Pearson, 2020). In addition to federal stakeholders, a host of stakeholders working under the High Desert Partnership are addressing restoration of wetlands on Malheur National Wildlife Refuge, as well as future management actions to cooperatively manage limited water resources in the basin. In short, there is a high level of "stakeholder readiness" (Halofsky and others, 2018) in the basin. In the framework of EarthMAP, this Use Case is very "demand-driven": it is highly certain that stakeholders would place high value on products that EarthMAP could deliver, and that these products would be integrated into decisions that influence outcomes for decades in the Use Case area. 


\section{Operational Implementation and Technical Considerations}

We envision products that EarthMAP can deliver for this Use Case would parallel other developments providing novel visualizations for science applications, for example, the National Institute of Visible Human Project. In short, application of EarthMAP in this Use Case could provide stakeholders with an analog of this project: the "visible riverscape." This product would allow stakeholders to view spatially and temporally variable meterological, hydrologic, and ecological conditions, as well as view the current social landscape, and to more readily envision scenarios and outcomes associated with alternative futures and management decisions. Technical capabilities to execute the Use Case exist but are not currently supported by existing funds. Additional resources would be required to further application of EarthMAP to leverage existing USGS and cooperator investments in this effort. In addition to the conduct of the science itself, additional support for time to fully engage stakeholders would represent an important investment. As mentioned previously a strong stakeholder establishment is present but allocating time to fully engage them is an important aspect of this work that is not currently supported. In addition to the existing team of scientists and support needed for their time, allocation of time or direct funding to support is needed to meet required technical elements (for example, AI/ML scientist, data scientist, and support for physically-based models) for EarthMAP. Determination of exact needs would require more detailed consultation on specific plans for implementation.

\section{Longevity and Sustainability}

The long-term viability of the science and technologies of this effort will depend on the initial investment in developing products, as well as periodic investments needed to provide ground-based and remote sensing information to update EarthMAP outputs, as well as data management and delivery of model-based products. Current decisions in the study area are being considered over longer (greater than $20 \mathrm{yr}$ ) timeframes (for example, drought adaptation planning), as well as seasonal to annual adjustments in more immediate actions (for example, decisions regarding agricultural water uses, livestock grazing, fish and wildlife management). If resources allow we could also incorporate an effort to quantify metrics of performance and success that specifically address the stakeholder community (for example, through interviews conducted by a social scientist (Delie and Biedenweg, 2019). More conventional measures of effectiveness (for example, assessments of model performance or validation) will also be conducted, but we consider success as judged by the stakeholder community as the ultimate measure of effectiveness. We strongly believe that technical approaches developed and lessons learned from application of EarthMAP in this Use Case can be adapted to the broader objective of applying EarthMAP across diverse social, ecological, and hydrologic settings across the Nation. The Use Case of the Donner und Blitzen River represents a unique opportunity to integrate all these elements and advance the grand challenges of the 21st century that EarthMAP seeks to address.

More broadly, over the next 3 to 5 years, the Use Case of the Donner und Blitzen river can provide lessons and a means of adapting EarthMAP to other basins currently under investigation across the Nation under the USGS Eco-Drought study, as well as within the next 10 years adapting these cases to extend across the western United States where transformational droughts are acting to influence ecosystem processes and human well-being (Crausbay and others 2020). Other agencies are supporting collaborative work with USGS and partners now to move in this direction and application of EarthMAP in this Use Case would provide a powerful means of enhancing these collaborations, relationships, and societal outcomes for adapting to the grand challenge of drought in the 21 st century.

Primary Contact: Jason Dunham, jdunham@usgs.gov

\section{Geography of Change-Synthesizing Multiple Dimensions of Anthropogenic Global Change with Indicators of Ecological and Hydrological Conditions}

We propose to 1) synthesize existing datasets describing the drivers of hydrological and ecological change; 2) model relationships among drivers and indicators of ecosystem condition; and 3) deliver reach-to-watershed assessments of hydrological and ecological trends and current condition status. Using readily available abiotic and biotic datasets from the Pacific Northwest Region (PNW), we will identify 'multidimensional refugia' and inform prioritization decisions across the landscape. 


\section{Demand and Relevance}

Interactions between different components of global change underlie may important ecological and economic impacts. For example, invasive species create fuel for fires whose intensity is increased by climate change, and newly intensive rainstorms fall on increasingly impervious land cover that intensifies flooding. Mapping and modeling spatial patterns of anthropogenic change - including climate change, land and water use change, and species invasions - is key for understanding and predicting variation in ecological and hydrological condition and function, and for anticipating and mitigating associated socioeconomic impacts. USGS data products capture key elements of resource condition and global change but we still lack an overarching framework to integrate, understand, and anticipate the interactions among different elements of global change. We propose to synthesize existing data products to describe the intensity of anthropogenic change and predict its implications for natural communities and ecological services. We propose to develop our Use Case with an initial focus on ecohydrological conditions in the PNW, while recognizing its applicability to terrestrial ecological systems as ecoregion boundaries complement watershed perspectives.

We will develop a platform to compile existing ecohydrological data and deliver assessments of ecological and hydrological condition to inform spatial prioritization decisions for water resources and species. Specifically, we would provide up-to-date and in-the-cloud compilations of environmental indicators, which are typically compiled in a laborious process for many individual National Environmental Policy Act (NEPA) and regional assessments by partner resource-management agencies and other stakeholders. Delivery of these compilations would be scalable depending on the targeted use (for example, individual stream reaches to regional HUC 4-6 watersheds). Our approach would reveal where multiple dimensions of global change intersect, and conversely, could identify areas that act as 'multidimensional refugia' for conservation and management prioritization. These refugia could be defined as areas experiencing low rates of multiple types of global change and (or) where system attributes are expected to minimize sensitivity. We would apply machine-learning and statistical analyses to characterize the linkages among datasets, quantify key interactions between the different elements of global change, and provide predictions and uncertainty bounds for data sparse areas. We anticipate that our synthesis would inform issue-specific analyses, such as updating threat assessments for salmonids, predicting algal blooms and their impacts, selecting indicators used in monitoring and adaptive management, and assessing trends and current conditions as necessary for NEPA assessments. We would develop our approach within the PNW, while ensuring it is scalable to the CONUS and ideally to Pacific Islands and Alaska (contingent on focal questions and datasets). We envision an interdisciplinary, spatially-explicit, and interactive online framework that delivers up-to-date information on ecohydrological status (for example, clickable summaries within HUCs) and helps decision-makers anticipate the impacts of anthropogenic changes within watersheds.

\section{Improved Decision-Support}

Our synthesis can inform a range of resource management planning that extends from local municipalities to federal agencies. We will provide nested grain sizes, from local (reach-scale) to larger watersheds, and analyses will be tailored based on stakeholder needs. Our aim is to co-develop our work with key partners (for example, BLM, USFS), while consulting with other users to ensure broad applicability and utility. Currently available tools for water resource assessments remain relatively siloed. For example, USGS Water-Quality Trends provides trends on many water-quality constituents based on discrete time series datasets, and Climate Engine is a powerful, in-the-cloud web application that delivers plots and spatial summaries. However, resource managers typically evaluate multiple datasets that can be difficult to align. In addition, many land managers may lack the technical background or resources to evaluate multiple datasets to understand trends and conditions to support the NEPA process. Following the conceptual framework of Climate Engine's analysis and visualization, we will integrate ecohydrological datasets for targeted stakeholder needs.

Our framework can inform local decisions by capturing the regional value of a given resource. For example, one application considers a National Forest developing a programmatic NEPA document for logging. Our models will have complied current conditions (land cover, presence of invasive species, water quantity and quality) and indicators of change (for example, rates of land-cover conversion; climate change; change in flow regimes, drought and fire hazard) to identify areas representing multi-dimensional refugia. These summaries would facilitate decision making within management units while also providing regional context, which represents an identified need and ongoing challenge for USFS partners. Moreover, decisions could be made rapidly, without the need to collect additional data or undertake laborious analysis. 


\section{Science Capabilities and Advancement Opportunities}

Existing datasets can include, but are not limited to the following portals: landcover (for example, National Land Cover Database, LANDFIRE, and FOREcasting SCEnarios of Land-use Change); water quantity (for example, NWIS streamflow, PROSPER streamflow permanence, NWIS groundwater levels, U.S. Drought Monitor, The Climate Toolbox); water quality (for example, NorWeST stream temperature, national water quality trends); and the composition of biotic communities (for example, USGS Nonindigenous Aquatic Species [NAS], eDNAtlas, USGS BISON, and BLM Assessment, Inventory, Monitoring [AIM]).

We have reached out to partners at the BLM to initiate discussions about the deliverables and use of our proposed platform. In a next phase of this project, we would expand discussions to include agency partners with whom our team has established relationships (for example, USFS, Bureau of Reclamation, state water resource departments) and we would reach out through our networks to new partners in state and federal agencies (for example, National Marine Fisheries Service), land trusts, conservation districts, and municipalities.

\section{Operational Implementation and Technical Considerations}

Our proposed Use Case would draw from existing human and technological capacity within USGS and we would broaden our team as needed. Data science workflows would utilize the APIs of established products. We would serve summarized information via a web-based platform, and to do so we envision leveraging existing data science and computer programming expertise within USGS (for example, the Oklahoma-Texas Water Science Center Integrated Hydrology and Data Science). We would apply statistical and machine learning models to understand the linkages between multiple sources of global change and indicators of biological and hydrological condition. Our team includes expertise in quantitative tools including statistical modeling, trend analysis and machine learning methods. One challenge we anticipate is that while some datasets provide spatially continuous information along streams and rivers (for example, NorWeST, PROSPER), many other temporally rich datasets are constrained to a point location. We would work with stakeholders to identify useful summaries of these point datasets, and could evaluate whether artificial intelligence tools can reliably predict ecohydrological condition based on a combination of continuous and point data. Our research team has experience designing and delivering novel products online, and we would reach out to include additional expertise in data management, other analytical approaches (for example, process-based deep learning), and visualization frameworks.

\section{Longevity and Sustainability}

Our Use Case will have long-term relevance because it can inform a broad set of management prioritization decisions, and can facilitate revisiting priorities as new information emerges. We aim to replace the piecemeal process of data aggregation from multiple sources by delivering up-to-date and easily accessible data summaries. We will draw from flagship USGS products that capture landcover (NLCD), streamflow (NWIS, PROSPER), and invasive species occurrences (NAS). By leveraging ongoing USGS investments, our Use Case will be more sustainable and scalable with extensions to the CONUS and Pacific Island systems. The emphasis on USGS products places our agency in a leadership role for providing aggregated information, and we will also draw from well-maintained additional sources of information (for example, for climate-change patterns and projections). We will measure the effectiveness of our effort by its use by stakeholders and by the impact on their decision making.

Primary Contact: Kristin L. Jaeger, kjaeger@usgs.gov

\section{Sustaining the Western Range: Actionable Science for Effective Management of the Sagebrush Biome}

Increasing threats to the ecological integrity of the vast sagebrush biome and the range of ecosystem services it provides have catalyzed an explosion of actionable science production by USGS over the past two decades, yet resulting products are not always readily discoverable or tractable for natural resource managers working across diverse and broad jurisdictional boundaries. This EarthMAP effort will integrate 21st-century multidisciplinary science co-produced across four USGS regions into data-delivery and visualization platforms that facilitate real-time decision making for DOI managers and their partners. 


\section{Demand and Relevance}

The sagebrush (Artemisia spp.) biome, which spans over 160 million acres across 13 western states and provides important ecosystem services and benefits to both wildlife and human communities, is widely recognized as being imperiled as it has contracted by over 50 percent since European settlement. Notably, over 350 plant and animal species depend on sagebrush rangelands for all or part of their life cycle, including sagebrush obligates like the greater sage-grouse whose declining populations and threats to vital habitat have prompted multiple status assessments under the Endangered Species Act. Approximately 70 percent of the biome is managed by federal and state agencies. Accordingly, land-use decisions affecting the biome largely drive complex conservation policy at national and state levels. Land managers spend millions of dollars annually on land treatments to counter the increasing threats from disturbances such as wildfire, invasive plants, drought, and woodland encroachment to sustain multiple use activities such as livestock grazing, mineral extraction, energy development, and recreation that can pose their own risks.

While contemporary research efforts steered by inter-agency collaborations such as the Integrated Rangeland Fire Management Strategy Actionable Science Plan (Integrated Rangeland Fire Management Strategy Actionable Science Plan Team, 2016) and resulting Science Frameworks have greatly improved understanding of sagebrush ecosystems' responses to disturbance and corresponding effectiveness of conservation and restoration techniques, there are clear opportunities to better incorporate past, current, and future knowledge into management practices. These efforts would build on nearly two-decades of co-production among Federal (DOI and non-DOI), State, and Local agencies, nongovernmental organizations, and the public across the West. To do so, USGS scientists across 4 regions (Northwest-Pacific Islands, Rocky Mountain, Southwest, Midcontinent) and multiple Mission Areas (Ecosystems, Energy and Minerals, Core Science Systems) comprising a Sagebrush Working Group (SWG) are primed to leverage multidisciplinary expertise spanning restoration, wildlife ecology, ecohydrology, + science, remote sensing, human dimensions, and advanced quantitative modeling. The EarthMAP framework will allow more effective integration of volumes of data describing ecological processes, disturbance histories, and management actions across the entire biome and over time periods ranging from decades to centuries. Formerly disparate, these data will form the backbone for multiple integrated predictive science tools and actionable intelligence. The underlying "engine" features enhanced spatiotemporal integration across ecological gradients and management jurisdictions and includes inputs for multiple prediction tools including data mining, hierarchical parameter estimation, and machine learning. User-friendly displays for decision-support will include creation of various treatment scenarios and corresponding real-time predictions based on underlying parameters that represent a host of rangeland ecosystem processes and services.

\section{Improved Decision-Support}

The SWG is developing science to inform planning and prioritization, conservation design and delivery, monitoring, and adaptive management. The science behind this Use Case will improve decision making at multiple scales, including placement of treatments on the landscape to maximize benefit to high value resources, and reduce conflicts between land uses, and selection of plant materials and actions that will minimize cost and maximize the effectiveness of the treatments.

Multi-scale and co-produced SWG science products forming a foundation for the Use Case are in high demand and widely used. For example, the National Landcover Database (NLCD) Fractional Shrubland Component and related 'Back-In-Time' (1985 and 2018) products allow biome-wide assessments of long-term changes in landscape condition and treatment effectiveness and identify potential conflicts between areas of high energy potential and wildlife habitat. These products coupled with predictive sage-grouse population habitat models inform implementation of the BLM/ USFS Sage-grouse Habitat Monitoring Framework and the Sage-grouse Habitat Assessment Framework and facilitate conservation planning for actions such as fuels reductions, post-fire restoration, and energy development. In collaboration with BLM, FWS, and State Wildlife Agencies across all 13 states, the SWG most recently developed a sophisticated Targeted Annual Warning System (TAWS) that allows managers to identify local sage-grouse populations in need of additional monitoring and management intervention when they fall out of synchrony with larger population aggregations. SWG scientists have also developed a suite of tools that provide the basis for modeled predictions and decision support for sagebrush restoration and land-use planning efforts. These include several web-based applications aimed at increasing data accessibility and co-production with managers. The Land Treatment Digital Library catalogs over 100 years of treatment information and is employed by the Land Treatment Exploration Tool (LTET) which mines its historic data to predict management outcomes in user-prescribed areas, generating a variety of spatial products that feed directly into planning documents. The Smart Energy web tool incorporates spatial information to better predict areas of potential fluid mineral and renewable energy development. Both the Smart Energy and LTET web tools display information from the Conservation Efforts Database (CED). The CED captures data from state and federal agencies and nongovernmental organizations describing the conservation and restoration efforts implemented in the sagebrush biome and can provide important context to evaluating restoration effectiveness. 
The SWG remains strongly engaged with multiple working groups through regularly scheduled conference calls and ongoing conversations to refine and update science needs. The latter includes lead coordination for the Integrated Rangeland Fire Management Strategy Actionable Science Plan (IRFMS) update to ensure the most pressing science needs of the management community are being addressed. The original Plan was developed in 2015/16 and the 5-year revisit of the plan has been initiated.

Going forward, the multi-scale framework and engaged SMG facilitates next-generation science and decision support tools that will be directly relevant to managers working to maintain and improve the condition of the sagebrush biome. This framework can lead to tangible benefits for the people and species that depend on this landscape, increase the efficiency and effectiveness of treatments, and reduce the long-term costs of actions necessary to achieve land management goals.

\section{Science Capabilities and Advancement Opportunities}

The USGS Sage-Grouse and Sagebrush Ecosystem Research Program (which comprises the SWG) includes scientists located in 11 westerns states from 3 of the 7 USGS Mission Areas and is led out of the USGS National Center, Ecosystems Mission Area. Research is purposefully aligned with priority needs outlined in the IRFMS, with DOI priorities as outlined in Secretarial Orders 3353, 3362, and 3372, and other science needs as they are identified by partner agencies.

This research can roughly be organized into five thematic areas: fire; invasive species; restoration; sagebrush, sage-grouse, and other sagebrush-associated species; and weather and climate. USGS research on these themes has resulted in a large and growing body of science that is meeting ongoing and emerging challenges faced by our stakeholders. Much of this work deals with the complex interaction of these themes; for instance, restoration of the sagebrush vegetation community, as is necessary following rangeland fire and extensive invasive annual grass invasions, requires precise deployment of limited resources to achieve the best possible outcomes. Working across multiple disciplines and stakeholders, USGS scientists are creating restoration tools that give early warning to managers to target actions in areas with decline sage-grouse populations, tools that enhance understanding ecological drought, and web applications to track ongoing investments and inform future management actions.

The newly completed Sagebrush Conservation Strategy provides a road map for the next 10 years of science and management interactions to resolve the grand challenges facing the sagebrush ecosystem. Using USGS and 21st-century science capacities we can improve the efficiency and effectiveness of land management actions, reverse or stabilized wildlife population declines, and improve the overall condition of western rangelands for the use and enjoyment of the American public.

\section{Operational Implementation and Technical Considerations}

The SWG has a demonstrated capability to develop actionable science tools by working closely with management partners to co-produce science that confronts existing and evolving challenges. In the process, they have built the foundation for the next generation of decision support tools that are informed by 'big data' and aimed at increasing the capacity to predict management outcomes. The suite of tools described in section 2, and others, deliver USGS science to management agencies in user-friendly interfaces that directly inform their decision-making processes. Other more recent products require further development to bring the science into operational forms. For example, the NLCD Fractional Shrubland Components and associated 'Back-In-Time' data are critical sources of information for multi-scale modeling across the working group, but real-time visualization tools to support management decisions are lacking. If integrated with the newly developed TAWS in an interactive web-based format, wildlife managers would have a highly valuable tool to identify where changes in land cover associate with declining wildlife populations, and better target land treatments. Existing and rapidly developing tools could be brought together into a more holistic framework of access to information across the spectrum and use the EarthMAP framework to better deliver USGS science. 
USGS, in coordination with BLM, USFWS, and the Western Association of Fish and Wildlife Agencies (WAFWA), has also been developing SageDAT, a mechanism for broader integration of data and mapping services that allows for broader participation and transparency in decision making among federal and non-federal partners. The SageDAT team is building upon USGS ScienceBase to allow effective sharing and discoverability of relevant data and tools while maintaining/preserving control by data owners. This makes a vital linkage to State, private, and nongovernmental data for the next generation of online tools. This system will also be an outlet for access and delivery of USGS data and tools. In addition, USGS web application development teams at FORT (Fort Collins Science Center) and FRESC (Forest and Rangeland Ecosystem Science Center) have successfully developed tools (see Section 2) to inform management actions. The team at FORT is pioneering the move of USGS to cloud-based web application development and brings significant capabilities to ongoing and future development. Despite these successes, teams will require additional resources to support development costs of the next generation of tools, as current support for ongoing operations and maintenance of the cloud-based tools remains a challenge and is an impediment to development of new operational tools. Most of the funding for these efforts is provided by management partners via project-base interagency agreements that have a limited time span. As USGS web-applications increase in complexity, programming capabilities and computational power will need to increase to facilitate advanced modeling, real-time processing, and the integration of larger data streams. Improved mechanisms to recruit and retain computer scientists and other IT-related positions will be critical for long-term success of the data and tool delivery component of this effort.

Overall, this effort is built on the continued advancement of science related to sagebrush ecosystem vegetation and wildlife responses to ongoing disturbances and management actions. The development of new tools will further hinge on improved field-based monitoring of vegetation conditions and wildlife populations. This could be through the development of new sensor technologies, refinement of auditory surveys for passerine birds, advances in genetic barcoding and eDNA technology for terrestrial species, ongoing mapping of human activities, and other important data streams to support the monitoring component. USGS research scientists continue to advance the assessment capabilities to help inform predictive models.

\section{Longevity and Sustainability}

Demands for services and resources provided by the sagebrush ecosystem, and subsequent reliance on their effective management, are increasing. Urban and suburban population growth, demand for energy (renewable and non-renewable), ranching and other agricultural uses, and consumptive and non-consumptive recreation will continue (and in some cases rapidly expand) into the foreseeable future, while the spread of invasive plants, devastating wildfires and prolonged drought are compounding the complexity of the management decisions to sustain these landscapes. For DOI, other agencies and private landholders responsible for the management of millions of acres in the West, decisions regarding placement and selection of land treatments to maintain and or improve the sagebrush ecosystems and mitigate threats remain an ongoing challenge.

By building on long-standing partnerships with the federal and state agencies, ranchers, and other NGOs tasked with resolving the growing array of resource conflicts, USGS can sustain long-term coproduction of the science that addresses managers' priority information needs. Success will require continued investment in the multiregional and inter-Mission Area SWG, engagement by USGS personnel at all levels of the agency with the appropriate DOI and state agencies, and stable fiscal support of the research and tool development identified by management partners. Reliable long-term fiscal support would facilitate research to develop the science to drive the next-generation tools and costs of web application development, operations, and maintenance. A sustained financial commitment from the USGS will demonstrate our commitment to our partners who have long supported this work, and who continue to request and use it.

Over the past decade, the land management agencies have put several monitoring systems in place, including the Sage-grouse Monitoring Framework, the BLM Assessment Inventory and Monitoring program, and National Resources Inventory, that can help track multiple metrics of success. These programs provide local- and landscape-level metrics of management effectiveness. Important metrics to follow will include changes in vegetation condition, costs of land treatments, size and intensity of rangeland fire, population size and viability of wildlife populations, and prevalence of requests to list species under the Endangered Species Act. 
Development of tools to increase the success of treatments in the sagebrush ecosystems can be directly applicable to semi-arid and arid rangelands throughout the western U.S. (Mojave, Chihuahua, Colorado Plateau, Sonoran), and throughout the world (for example, Argentina, China, Mongolia, East Africa). The decision analysis frameworks proposed here will be scalable to other at-risk species and ecosystems. For example, the greater sage-grouse TAWS can provide a framework for any species with expansive range and consistent monitoring information; treatment evaluation tools can be established if information on prior treatments are available for a particular region. The partner engagement in the identification of science priorities as outlined in the IRFMS, the co-production of multi-agency and interdisciplinary research that feeds directly into management guidelines as demonstrated in the Science Frameworks, and the integration of massive amounts of data and information as is pursued in SageDAT are highly successful and noteworthy models that can inform similar efforts to evaluate the success of complex land and species management issues across the globe.

Primary Contact: Lief Wiechman, lwiechman@usgs.gov

\section{Surface-Water Index of Permanence for Ecohydrology (SWIPE)}

The presence and absence of surface-water flow across landscapes over time affect water quality and availability, which have implications for the protection and management of human and ecosystem health. We propose to integrate expertise, methods, and data from multiple USGS Mission Areas to develop a Surface-Water Index of Permanence for Ecohydrology (SWIPE) for the purpose of providing actionable intelligence to ecosystem-resource managers, and to better understand two key ecological mechanisms: the spread of invasive species and the transmission of pathogens.

\section{Demand and Relevance}

Warming temperatures as a result of climate change are predicted to increase drought severity and cause shifts to aridity across the United States (Martin and others 2020; Overpeck and Udall, 2020). It is increasingly recognized that understanding trends in persistence of surface-water features on the landscape will be crucial for water-resource managers. Surface-water features, such as headwater streams and springs, are particularly important as they serve as the primary interface between terrestrial and aquatic species and systems and can function as ecohydrologic refugia - areas that remain buffered from changing hydroclimatic conditions over space and time - in otherwise water-limited landscapes. However, the vulnerability of these ecohydrological refugia to changing climatic conditions are poorly understood. The paucity of existing, consistent datasets representing surface water-permanence has historically limited investigations of changes in surface-water availability over multiple time steps. Further, because of this data gap, scientists have previously been unable to investigate how these changes in surface-water availability and timing affect wildlife behavior and ecosystems services. Understanding this relation has implications for management strategies, including (i) areas to focus efforts for invasive species and pathogen preventions and (ii) elucidating the consequences of restoring connectivity among aquatic systems. While this Use Case will focus on studying the link between surface-water permanence and the spread of diseases and aquatic invasive species, the resulting data from this work will facilitate investigating the role of surface-water availability across a much broader array of ecohydrologic and socioeconomic systems (fig. 5.8).

\section{Improved Decision-Support}

This EarthMAP project seeks to provide managers, decision makers, and the public with actionable information at multiple relevant spatial and temporal scales about surface-water permanence, stream-network connectivity, and ecosystem effects. Surface-water permanence, which includes headwater streams and groundwater expressed on the surface as springs, drives many management decisions regarding water-quality standards, water rights, and land use. These management decisions are constrained by available tools that neither adequately capture the year-to-year variability in the location and duration of flowing streams, nor describe their connectivity between locations. Consequently, there is a need for up-to-date surface-water permanence classifications that reflect both average conditions, as well as the year-to-year changes across larger extents and at multiple scales. This information would be extremely informative for water-use management. For example, clean and abundant water is an identified need for livestock grazing, energy development and other multiple-use categories defined in Section 103 of the Federal Land Policy and Management Act of 1976 (U.S. Department of Interior, 2016. Therefore, improving understanding of surface-water permanence is necessary for effectively managing public lands and ecohydrologic resources for sustainable multiple use. 


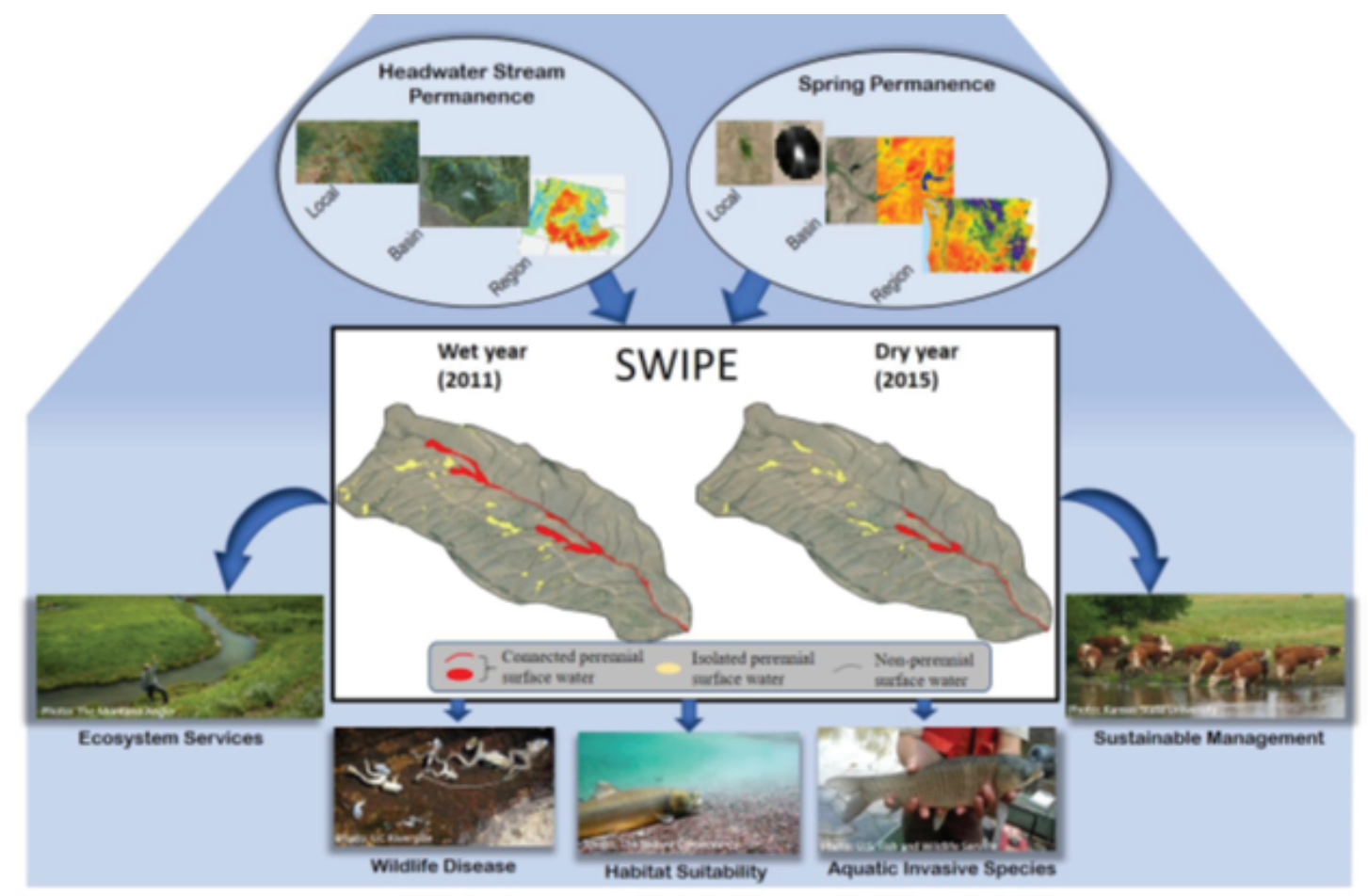

Figure 5.8. Diagram of data incorporation to develop a surface-water index of permanence for ecohydrology (SWIPE) and the potential ecological applications for partners and managers.

\section{Science Capabilities and Advancement Opportunities}

The USGS has developed multiple modeling techniques that are designed to predict the likelihood of streamflow permanence and spring permanence using state-of-the-art, data-driven methods including remote sensing and machine learning. For example, the PRObability of Streamflow PERmanence (PROSPER) is an empirical, machine-learning based model that predicts the probability that locations on streams will retain surface flow through each year. Additionally, the USGS has done work to develop a remote-sensing method for detecting the sensitivity of springs to climate change. Both of these approaches have been used at different scales in the Pacific Northwest. By combining output from these methods, or recalibrating the methods to incorporate aspects of both approaches into a more holistic watershed model, we are creating the opportunity to understand interannual changes in surface-water availability at an unprecedented temporal granularity and in a regionally consistent manner. Further, we plan to implement state-of-the-art sampling techniques, including the use environmental DNA (eDNA), which allows us the capacity to quickly and cost-effectively sample and detect the presence of species in a watershed. 


\section{Operational Implementation and Technical Considerations}

A logical path forward with this work is to implement a three-phase plan to investigate surface water-permanence and its relationship with invasive species and pathogen spread. Phase 1a: Utilization of currently available datasets to develop perennial surface-water connectivity maps. In phase 1, we will collate existing USGS products, including the PROSPER model (Jaeger and others, 2019) and a remote-sensing approach for evaluating spring vulnerability to climate change (Cartwright and Johnson, 2018) to produce a probabilistic index of ecohydrologic refugia (surface-water permanence) at biannual time steps within a watershed (HUC-10; see fig. 1. Phase 1b: Development of risk maps based on current data and understanding of aquatic invasive species occurrence and pathogen transmission. Congruence between SWIPE and aquatic invasive species and pathogen occurrence data will inform machine learning algorithms, which aim to predict the probability of invasive species or pathogens introduction, existence, and spread. Risk predictions will be ground-truthed using strategic eDNA sampling methods to detect presence of the target species. Sampling will be performed twice per year, at high- and low-flow periods in aquatic areas that are predicted to be connected, as well as a subset of aquatic areas that are predicted to be isolated. We will demonstrate these methods through two use case assessments, the first addressing an invasive species (for example, grass carp, Ctenopharyngodon idella) and the second addressing an emerging infectious disease (for example, amphibian chytrid fungus, Batrachochytrium dendrobatidis). Phase 2: Cloud-based interactive mapping tool for managers with capacity to incorporate new datasets. Products from phase 1 will be implemented through a tool on a cloud-based platform, with intent to publish the tool for public use. Phase 3. Predicting future spread of pathogens and invasive species. Using the probabilistic index of surface-water permanence from phase 1, we will forecast stability of these surface-waters under three climate change scenarios: (i) increased temperature and stable precipitation, (ii) increased temperature and decreased precipitation, and (iii) increased temperature and increased precipitation. Using these forecasts for surface-water permanence, risk maps will be created to identify refugia and areas where disease and invasive species have potential to spread based on connected surface-waters.

This work will require key experts across multiple disciplines and USGS Mission Areas. Specifically, data scientists, remote sensing specialists, machine learning experts, aquatic ecologists, disease ecologists, hydrologists, and potentially others. Because of the amount of data that will be processed and analyzed, the majority of the work will be done on the USGS High-performance Computing systems (YETI, Tallgrass, Denali), and will also leverage a cooperative agreement that is being developed between the USGS, Google, and Desert Research Institute. Many of these resources/ collaborations are already in place and can easily be leveraged.

\section{Longevity and Sustainability}

The products from this project will be developed in a modular manner with the capacity to (i) be applied and (or) expanded to multiple scales ranging from local (stream reach) to regional, and (ii) incorporate new data streamsspecifically, new invasive species or pathogen occurrence data - to create risk maps for novel and on-going threats. All data and models will be developed and based in the cloud to minimize maintenance and the potential for abandoned and broken services. Finally, as the rate of climatic change is predicted to be significantly higher than in the past, the threats from water-related stressors and their effect on invasive species and pathogen dynamics will increase. Because of this, we anticipate the products of this project to have immediate national application and interest that will only increase in value as the impacts of climate change, increased water use, and more frequent human/wildlife interaction are realized across the country.

Primary Contacts: Alynn Martin, alynnmartin@usgs.gov; Lindsey Thurman, lthurman@usgs.gov; Roy Sando, tsando@usgs.gov; Joseph Kennedy,jjkennedy@usgs.gov

\section{From Water to Wildlife: Linking Water Timing and Availability to Subalpine and Alpine Vegetation and Wildlife Populations}

We will use existing datasets and cutting-edge modeling techniques to predict responses of vegetation and wildlife to the effects of changes in water quantity, availability, and seasonal timing in the subalpine and alpine regions of the northwestern United States. The framework and predictions developed here will create a template for integrating water and wildlife in other ecosystems, directly serve DOI partners, and provide information and strategies for neighboring landowners and agencies. 


\section{Demand and Relevance}

Water timing and availability directly and indirectly influence terrestrial wildlife and their habitat, yet linking these explicitly is rare, even where data exists. Managers need to better understand these connections to protect, maintain, and restore key wildlife and plant populations and the ecosystems to which they belong. Subalpine and alpine systems in the mountainous western United States are highly vulnerable to climate change, but provide opportunities for water, vegetation, and disturbance management that could greatly affect multiple species both in these communities and downstream. By integrating existing datasets (for example, representing snowpack, surface water, evapotranspiration, glaciers, avalanches, fires, soils, vegetation, and wildlife), we will predict vegetation and wildlife responses to the effects of changes in water flow, availability, and seasonal timing in the subalpine and alpine regions of the northwestern United States. Our initial focus will be on alpine and subalpine National Park Service units in Washington and Montana, where our team has existing connections with decision-makers tasked with conserving and managing wildlife population and ecosystem function. However, we envision predictive modeling results to be relevant across multiple land management jurisdictions and ecoregions. We will map changes in subalpine and alpine meadow size, extent and vegetation composition as a function of shifting surface and subsurface water and evaluate subsequent effects on wildlife distribution and population dynamics. In this effort, we will integrate water, vegetation, and wildlife data (across multiple trophic levels) for new insights; synthesize patterns in National Park units across the northwest; and provide a template for broader use in connecting water to wildlife from local scale management questions to regional trends. This effort is unique in that prior links between water and wildlife have focused primarily on aquatic species.

\section{Improved Decision-Support}

National Parks have a mandate not only to preserve individual species, but also to maintain ecological functioning and integrity in changing ecosystems. As such, national parks have an immediate need for understanding relationships among water, vegetation, and wildlife in order to plan proposed infrastructure (for example, cell towers), backcountry visitor use, weed management, and prescribed fire, and to manage for species of concern including invasive species, disease, aquatic insects, bumblebees, pikas, marmots, mountain goats, and grizzly bears. Our team has close, long-term connections with National Park personnel that will ensure that such work will improve decision making and focus on key deliverables. Our products will provide park-specific recommendations while also determining patterns of change across mountain systems that will be relevant not only to park stakeholders but also to surrounding landowners and resource managers. Our products will help identify existing climate refugia in subalpine and alpine systems, as well as areas that may require active management. While the first phase of this work is focused on select National Parks, the need to manage wildlife and its interaction with humans is universal and a goal of most federal, tribal, and state agencies. Ultimately, the framework developed here will be readily applicable in other, lower elevation ecosystems where managers are faced with similar questions and management challenges. The results of the expanded study would be informative to issues such as human/predator conflicts, effects of development on migration routes, changes in ecosystem carrying capacity related to climate, changes in ecosystem services and economic value, and many others. 


\section{Science Capabilities and Advancement Opportunities}

This Use Case will leverage existing but disparate datasets to produce a novel, cross-disciplinary analysis that explicitly links key water quantity and timing metrics to effects on vegetation and wildlife. First, we will work with wildlife, snow, and other locally collected datasets contributed by Park partners in the region to establish a proof-of-concept within select National Parks. We have initiated conversations with partners in multiple National Parks (Olympic, Mount Rainier, and Glacier) who are excited to integrate local datasets with remotely sensed and (or) regional datasets. Working collaboratively with stakeholders to develop the project more fully and incorporate their knowledge and available data will be key to designing and developing products useful to biologists and managers. Regional hydroclimatic datasets (for example, remotely sensed snow products, and gridded precipitation, temperature, evapotranspiration, etc.) will be utilized to capture the mechanisms driving the changes seen in vegetation and wildlife distribution and use. We will incorporate modeled (for example, PROSPER, PRMS, NWM, others) and observed (USGS streamgages, citizen science applications such as FLOwPER, others) hydrologic data to inform our models on the availability and timing of surface water flow. Finally, we will rely on remotely sensed data developed by USGS and other agency partners (for example, LEMMA on vegetation cover and photosynthetic activity, Berman and others (2020) on changing vegetation phenology and productivity, Manier and others on soil moisture /evapotranspiration, DHSVM [Distributed-Hydrology-Soil-Vegetation-Model]) to link water and snow data to vegetation. By linking these datasets, we can evaluate the direct effects of both water and vegetation on focal wildlife species to better understand mechanisms and potential management options for reaching wildlife management goals. Example focal species with existing datasets include pikas, Western bumble bees, mountain goats, Olympic marmots, and grizzly bears. While the initial project would be focused on select National Park units, we would ultimately reach out to other potential partners, as the results of this study would be broadly applicable, and explicit linkages between water and wildlife remain an important and understudied aspect of ecosystem function across the US.

\section{Operational Implementation and Technical Considerations}

This Use Case draws on our team's varied expertise within the USGS and existing relationships with National Park unit scientists and managers. Our team includes scientists with technical expertise in wildlife and plant ecology, remote sensing applications for hydrology, surface water-groundwater interactions, and relevant statistical analyses and modeling. Our strong relationships with National Park partners will enable access to key datasets and has informed the Use Case development. We will work closely with stakeholders to ensure that our data products meet their needs. We further plan to broaden our team to include additional partners with technological (for example, machine learning) or ecosystems (for example, fire, glacier and avalanche, additional remote sensing) expertise as appropriate. Other areas we anticipate needing additional capacity include expertise in data management and visualization, certain analytical approaches (for example, some aspects of machine learning), and effective, accessible delivery of products over the long-term (for example, online portal).

We anticipate several products from this project. (1) We will develop models quantifying the relationships between water quantity and timing metrics, vegetation indices, and wildlife seasonal use and distribution. These model results will provide immediate insight to park stakeholders on direct and indirect drivers of wildlife occurrence, which will aid in current management decisions. (2) We will then build models and create maps showing predicted changes in the quantity, composition, and quality of key habitats such as subalpine and alpine meadows under different climate change scenarios (that is, modeled changes in key hydroclimatic drivers). This modeling and mapping effort will identify climate refugia and threatened habitats within the focal ecosystems, which can guide active management and conservation efforts. (3) We will extend these models to show predicted changes in indicator and flagship wildlife species use, distribution, and abundance in meadow habitats under these same scenarios. These models will allow parks to understand the spatiotemporal characteristics of wildlife occurrence in new and integrated ways. Once the integrated models and predictive algorithms have been developed for this National Park case study, they can be adapted to other ecosystems of interest for local to regional applications. 


\section{Longevity and Sustainability}

Understanding linkages and relationships among water, vegetation, and wildlife are key to projecting and assessing ecosystem integrity, resiliency, and adaptability in diverse ecosystems under climate scenarios that forecast increasing temperatures, changing precipitation regimes, and changes in the frequency and magnitude of extreme weather events. Our Use Case will provide immediate patterns and predictions relevant to DOI stakeholders in National Parks tasked with conserving, maintaining, and restoring viable populations of listed species and key habitats. The tools developed here will help with decision support in the near- and long-term in these fragile subalpine and alpine environments and new or updated datasets can be incorporated over time. More broadly, the tools developed here will be scalable and transferable to (1) high-elevation ecosystems found in other land-management jurisdictions, and (2) other ecosystems where understanding linkages between water availability, vegetation, and wildlife is critical to effective decision making under future climate change scenarios. Integrating data from water to wildlife will have broad application for understanding wildlife and vegetation connections with and responses to climate-related changes in fire regimes, snowpack, and precipitation.

Primary Contact: Rebecca McCaffery, rmccaffery@usgs.gov

\section{Rocky Mountain Region}

\section{Integrated Science to Enhance Prediction of Post-Fire Hazards, Risk Assessments, and Decision Making}

We propose an interdisciplinary science integration effort for EarthMAP that crosses the Ecosystems, Core Science Systems, Natural Hazards, and Water Resources Mission Areas with external partners from the wildland fire community to align and improve USGS's diverse risk assessment and prediction capabilities.

\section{Demand and relevance}

Year after year western communities are made painfully aware of expensive and long-term economic and social impacts caused by wildfires, including the loss of property, livelihoods, and lives. Economic impacts alone amount to annual costs of $\$ 5$ billion for fire suppression and more than $\$ 80$ billion in direct and indirect losses. Yet fire in wildlands is essential to reduce future wildfire risk, improve wildlife habitat, maintain or restore ecological resilience, and manage fire-adapted species in many ecosystems across the Nation. Finding balance between wildfire benefits and risks requires information to support decision making and evaluate management alternatives. The Wildland Fire Leadership Council (executive level wildland fire representation of DOI, USDA, DHS (Department of Homeland Security), DoD (Department of Defense), States, Tribes and Communities) identified tools to assess vulnerabilities and mitigate direct and indirect fire risks before, during and after wildfires as one of the greatest unmet needs. A critical need is integrated predictive models for fire occurrence and severity, and potential for post-fire debris flows and changes in water quality and quantity. This need could be met by integrating fire science across USGS disciplines in a dynamic "fire risk" map that combines the:

- Likelihood of fire occurrence and spread, and the predicted severity of a fire;

- Immediate and near-term impacts of a fire on social and ecological resources (based on integrated assessment and modeling of how landscapes will change after fire);

- Severity of post fire risks and effects that result from fire-induced changes (based on that integrated assessment and modeling, supplemented by long-term predictive modeling); and

- Post-fire recovery and timing (based on fire severity and predictions of future conditions).

Such integrated assessment and prediction capabilities would enhance the ability of the wildland fire and emergency management communities to protect the safety, health, and prosperity of communities, minimize economic losses and ecosystem damages, and enhance post-fire mitigation and recovery.

The USGS is perfectly positioned to meet this need. Our expertise in fire and hazard-related sciences is well recognized by stakeholders that work with USGS to address challenges in wildland fire science and management across the Nation. However, USGS's many fire science capabilities require integration in order to support truly actionable management applications and decision making. We propose an interdisciplinary science integration effort for EarthMAP that crosses the Ecosystems, Core Science Systems, Natural Hazards, and Water Resources Mission Areas with external partners from the wildland fire community to align and improve USGS's diverse risk assessment and prediction capabilities.

The work we propose to integrate is designed to address the needs of our diverse stakeholders and aligns with agency, department, and national research strategies and management priorities:

1. The USGS Wildland Fire Science Strategic Plan (Steblein and others, 2021) priority \#1: Produce state-of-the-art, actionable fire science, although the other 3 priorities are also applicable. 
2. The Department of Interior's FY2018-22 Science Strategy Protecting our people and the border cross-cutting Mission Area \#5, specifically goals \#3 Manage wildland fire to reduce risk and improve ecosystem and community resilience and \#4 Provide science to safeguard communities from natural hazards through strategies that integrate fire ecology, risk management, and collaboration to mitigate wildfire impacts and monitor and assess natural hazards risk and response planning.

3. The National Cohesive Wildland Fire Management Strategy, which prioritizes balancing the ecological benefits of fire with active management and technological support to reduce wildfire risk.

4. Executive Order 13855, Secretarial Orders 3372 \& 3374, which set targets for land management and fuel reductions to reduce risk.

\section{Improving Decision Support}

Our stakeholders include DOI, USDA, DHS, DoD, States, Tribes and Communities represented by the Wildland Fire Leadership Council and involved with managing and mitigating the impacts of wildfires. Our science-based fire risk map will provide stakeholders with actionable intelligence by producing spatially consistent and detailed information about the potential for post-fire risks that are not currently available. Creating this "EarthMAP on Fire" vision will require close collaboration with the fire management community, to ensure that the results are truly actionable, and with the fire science community, to ensure that the best available science, is incorporated. We will work with the Wildland Fire Leadership Council and stakeholders to co-develop our approach, ensure the approach is useful for their needs, and fully understand how it would inform decision-making processes. Examples of where our risk map could inform decision making include: (a) activities to reduce the likelihood and severity of fires; (b) real-time fire suppression priorities targeted to areas where the impacts are of most concern, or where fire recovery would be most difficult; and (c) assessment of post-fire risks and recovery challenges to speed the deployment of rapid responses, such as debris flow management or revegetation and invasive species management. The post-fire decisions of fire and emergency managers will be further supported by near-real time updating of the fire risk map based on remote sensing observations of changes in the burned areas and by later monitoring and observation.

\section{Science Capabilities and Advancement Opportunities}

The USGS has many fire science capabilities. These include monitoring efforts for land cover (Land Change Monitoring, Assessment, and Projection [LCMap] and National Land Cover Database [NLCD]), vegetation (Gap Analysis Project [GAP] and LANDFIRE), fuels (LANDFIRE), fires (Monitoring Trends in Burn Severity [MTBS] and Landsat Burned Area), fire occurrence, and burn severity modeling, as well as, efforts to model fire occurrence and smoke emissions and post-fire geological and hydrological impacts. However, these capabilities are not yet integrated in a way that allows us to produce a fire risk map or to answer the management challenges described above. Getting there-achieving the goal of predictive, integrated fire-climate-ecosystem-hazards models that link landcover, fire risk, fire behavior, post-fire hazards, and short-and long-term fire effects, including ecosystem resilience and future fire risk - requires everything that EarthMAP aims for. It requires existing and new monitoring, with data made available rapidly, integrated across disciplines, and accessible to scientists and decision makers alike. It requires integrated and predictive models to evaluate the potential consequences of fire as part of the fundamental "pre-fire" risk assessment, and models that can evaluate post-fire conditions and highlight effective recovery options.

\section{Operational Implementation and Technical Considerations}

This effort aims to assess and reduce the risk of post-wildfire hazards such as flooding, debris flows, and water-supply impairment. While there is much existing USGS research that could be built upon, central to this effort is the need for continued research to develop more sophisticated, complex, and informative models that improve assessment and prediction of potential fire occurrence, behavior, hazards, and risks, as well as post-fire effects and recovery. Improved modeling capability relies on integration of fire, water, and geomorphology models with complex, $3 \mathrm{D}$, dynamic, multi-scale input data. This effort is organized around four objectives to provide an integrative simulation modeling framework.

Objective 1-Develop simulation models to predict fire behavior, burn area, and burn severity: 
The first step of this effort would link models that simulate and predict fire ignitions, fire behavior and perimeters, and burn severity to provide necessary inputs for post-fire hazard models. To this end, we will first assess the degree to which existing fire behavior and prediction models can generate these inputs. In particular, fire intensity has been used as a surrogate for fire severity in a previous debris flow modeling effort, but the relationship between modeled fire intensity and observed burn severity has not yet been assessed; if this relationship is poor, new methods for predicting burn severity will be developed. Our burn severity models will predict both classified and continuous measures of burn severity, as well as modeled correlates of field-based severity to allow for field measurements and validation. Coupling our burn severity models with ignition probability and fire behavior models will allow us to generate thousands of simulated fires which can be aggregated to provide probabilistic risk estimates of fire hazards.

Objective 2-Link fire model predictions of burn area and severity to debris flows models:

We will link fire model predictions developed in Objective 1 with debris flow models following the same operational approach used by the USGS to assess debris-flow hazards after observed fires. The USGS currently delivers post-fire debris-flow hazard assessments at the request of state and federal Burned Area Emergency Response (BAER) teams. Observations of burn severity are a critical input to these models. To produce an assessment of debris-flow hazard and risk that can be used to inform management decisions before a wildfire occurs would require predicted burn severity. In our integrated simulation framework, predicted fire perimeters and burn severity will be used as inputs to empirical models to estimate debris-flow likelihood and volume. Linked wildfire-debris flow models will be used to identify drainage basins with high debris flow risk, information that can be used by land management agencies to target fuels reduction or fire management activities that could potentially reduce burn severity and related post-fire hazard.

Objective 3-Link fire model predictions of burn area and severity to water models:

We will initiate systems development necessary to link predictive fire products with the USGS StreamStats program, a web-based application that is used by stakeholders to obtain watershed areas and characteristics and streamflow statistics (for example, flood flows, mean flows, low flows). StreamStats is currently developing a national application which will incorporate historical and active fire data (burn severity and fire perimeters from the Monitoring Trends in Burn Severity Program and National Interagency Fire Center, respectively) to identify water supplies that may be affected by wildfire runoff and to enable assessment of post-fire peak flows based on existing regression equations derived from post-fire runoff studies. We propose to supply simulations of burned area and severity to StreamStats from the models developed in Objective 1 to enable the assessment of potential risks of flooding and water supply impairment before wildfires occur. Such predictions would be useful to BAER teams and could enable at-risk communities to mitigate flood risks preemptively. Objective 3 activities would also enable the WMA (Water Resources Mission Area) to identify potential locations for immediate installation of streamgages and water-quality monitoring instruments, should a fire occur. This would enable both an alert system to downstream communities to prepare for and mitigate against flooding and water-supply impacts, and also provide model input to ongoing WMA efforts to predict impacts of extreme events on water availability.

Objective 4-Developing decision support functionality:

Through stakeholder engagement, we will determine what decision support functionality is desired and add capabilities incrementally. Because our approach operates on individual simulated fire perimeters, we will work with the wildland fire management community's IT organization to integrate our framework into the Wildland Fire Decision Support System (WFDSS). This would allow WFDSS users to generate near real-time predictions based on known ignitions at watershed or basin scales, directly supporting BAER teams.

\section{Longevity and Sustainability}

Sustained relevance of this Use Case is anticipated to be high as wildfire occurrence is expected to increase in the future given past legacies of fire suppression and projected patterns of development, and climate change. Furthermore, post-fire vegetation regrowth may also be limited or transition to novel ecosystem types because of continued drought conditions. Sustained long-term investment of this Use Case through USGS supported for research, stakeholder engagement, and computing infrastructure would enable:

1. Expanding the geographic range of the post-fire risk decision support system to the western US or beyond, including the enhanced components described below.

2. Expanding the capabilities and scope of the integrated modeling framework with complex ecosystem-fire process models to enable evaluation of fire-driven impacts at short- and long- time scales and assess the role of potential mitigation efforts (for example, fuels treatments) and post-fire vegetation recovery in attenuating risk.

3. Assessing fire season weather predictions to create seasonal scenario predictions of post-fire debris and flood hazards and application of integrated assessment and prediction capabilities to other fire-related issues, vulnerabilities, and hazards (for example, risk in the wildland-urban interface, risk, endangered species, wildlife habitat, etc.).

Primary Contact: Paul Steblein, psteblein@usgs.gov 


\section{Daily and Seasonal Forecasts of Groundwater and Surface Water Availability and their Interactions in the Upper Colorado River Basin}

Our aim is to provide an operational model of integrated hydrology of the Upper Colorado River Basin (UCRB) that provides continuously updated daily, monthly, and seasonal forecasts of groundwater and surface water flows and their interactions at fine scale $\left(1 \mathrm{~km}^{2}\right)$ resolution. These predictions are critical for managing drought and quantifying water availability for ecological and human water needs.

\section{Demand and Relevance}

The growing population of the arid and semiarid Southwest relies on overallocated surface water resources and poorly quantified groundwater resources (fig. 5.9). To meet human water needs, water managers must meet water delivery requirements while planning for future water supplies. Recent studies have found that about 50 percent of the surface water at USGS stream gages in the UCRB is derived from groundwater as base flow. Water resource management has focused on securing the surface water supply, and current water resource models simplify groundwater processes such that surface water is disconnected from groundwater in aquifers. Such simplifications reduce the ability of the models to represent base flow and complex interactions between groundwater and surface water in response to evapotranspiration, groundwater pumping, and their feedbacks in a variable climate and during drought. As a result, simulations of water resources and the impacts of variable climate on groundwater and surface water remain uncertain. We aim to improve water resource tools by providing daily and seasonal forecasts of water availability using continuously updated simulations of physically-based groundwater flow and surface water as a single, integrated resource.

In 2018, the USGS Water Resources Mission Area (WMA) and the Water Availability and Use Science Program launched an investigation of groundwater and surface water as an integrated resource in the Upper Colorado River Basin and parts of the Lower Colorado River Basin (Rocky Mountain and Southwest Regions). This ongoing study, called the Colorado Plateaus Aquifers: A Water Availability Assessment of Groundwater and Surface Water as a Single Water Resource project, here called the "Colorado Plateaus Project," will complete a model of integrated groundwater and surface water in Fiscal Year 2022 using the USGS software GSFLOW (Coupled Groundwater and Surface-Water Flow Model). An advantage of GSFLOW over hydrologic models by other agencies is that it simulates groundwater and surface water as an integrated resource. That is, it simulates how surface water is affected by changes in aquifer flow from groundwater pumping and human water use, changes in evapotranspiration, and changes in groundwater discharge as baseflow. GSFLOW also simulates runoff and snow melt contributions to groundwater recharge and to streamflow. These hydrologic couplings allow for rigorous representations of watershed hydrology with groundwater and surface water interactions. We are currently using the GSFLOW model to address the following questions.

- How have human water use and climatic conditions impacted groundwater resources, and what are possible future impacts on water availability in the Southwest?

- How might changes in groundwater availability affect surface water resources in the Colorado River Basin in the future?

- Can we reduce the uncertainty of future water resources in the Southwest with better understanding of the contribution from groundwater?

The Colorado Plateaus project, upon completion, will have a daily, high spatial resolution $\left(1 \mathrm{~km}^{2}\right)$ model ready for operational expansion for EarthMAP to address the above questions and stakeholder needs. This expansion is not part of the existing project scope. Our model complements new USGS efforts aimed at innovative hydrologic monitoring and integrated hydrologic modeling and prediction capabilities in the Upper Colorado. The WMA's new programs, Next Generation Water Observation Systems (NGWOS) and Integrated Water Availability Assessments (IWAAs), are in the initial phases of planning and implementation in the UCRB. Our model is planned to be a key tool in the water availability strategy in the IWAAs UCRB project, in collaboration with surface water modeling by the National Weather Service (NWS), National Center for Atmospheric Research (NCAR), and the Bureau of Reclamation (Reclamation). Our model could be used to improve estimates of salinity in streams by Reclamation's Colorado River Simulation System. Our forecasts could also be used by the Bureau of Land Management's Regional watershed modeling efforts related to salinity and rangeland impacts with the APEX (Agricultural Policy/Environmental eXtender) model. The Colorado River Salinity Control Forum needs model predictions of surface flows to improve predictions of salinity transport mechanisms from groundwater. Simulated streamflow may benefit U.S. Fish and Wildlife in assessments of ecosystem services. 


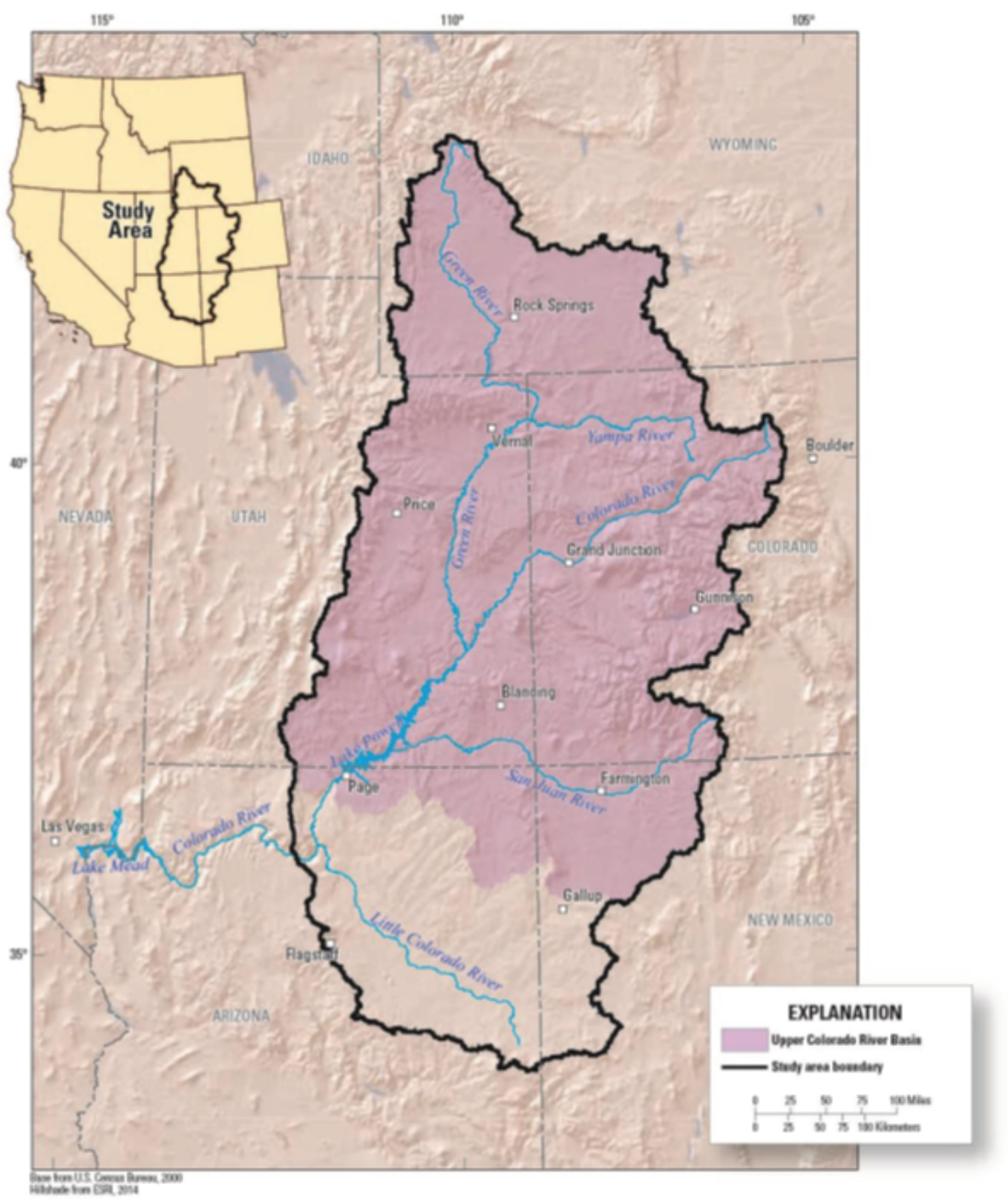

Figure 5.9. Map showing the extent of the Colorado Plateaus project and GSFLOW integrated groundwater and surface water model (black outline) and the Upper Colorado River Basin.

\section{Improved Decision-Support}

The Colorado Plateaus GSFLOW model can provide actionable and timely forecasts of integrated hydrological conditions across the UCRB, a product that is not currently available to decision makers or scientists. We will expand the capabilities of the GSFLOW model to provide forecasts from the National Oceanic and Atmospheric Administration (NOAA) Climate Forecast System which is updated daily (up to 28-day outlook) and monthly (6-month outlook). Such forecasts are needed for both water management and scientific understanding of hydrologic and ecological processes. Water use and demand forecasts may be provided by local and federal agencies (Reclamation) and the USGS Water Use model. The resolution of the forecasts will be daily through time and at spatial resolutions of $1 \mathrm{~km}^{2}$. Daily temporal resolution is fine enough to represent the dynamics of fast hydrologic responses to large precipitation events from storms, the accumulation of snowpack, and rapid snowmelt and runoff to stream channels. The $1 \mathrm{~km}^{2}$ spatial resolution resolves dynamic groundwater and surface interactions and baseflow throughout the UCRB. 
Forecasts at these timeframes are highly sought by Federal, State, and local resource managers as there may be enough actionable lead time to design and implement management strategies to address periods of excess water or drought. For the science community, the GSFLOW model provides a conceptual understanding of the hydrologic system and a science-support tool for evaluating the possible effects of human water development and climatic stresses on ecohydrologic systems. For example, our GSFLOW model has been identified as a potential tool for identifying jurisdiction of intermittent water for the Clean Water Act by the interagency effort on the Waters of the United States which includes the USGS, EPA, and U.S. Army Corps of Engineers.

This Use Case may generate new collaborations with stakeholders such as Reclamation and NWS. Reclamation currently provides essential river forecast products for the UCRB. However, their forecast models contain simplified groundwater and baseflow processes. There is opportunity to collaborate directly with Reclamation to evaluate and possibly improve model performance. Our GSFLOW model can fill gaps in predictive capability by simulating temporal and spatial changes in low and intermittent streamflow during drought periods that are sustained mainly by baseflow. The model can resolve the locations of these vulnerable stream reaches at 1-km resolution and forecast how wet and dry reaches may change seasonally.

\section{Science Capabilities and Advancement Opportunities}

The Colorado Plateaus GSFLOW model provides a foundation for providing actionable forecasts of groundwater and surface water conditions. Extensive existing data, software, and infrastructure have already been developed by the USGS and specifically by the Colorado Plateaus project to construct the model. However, this Use Case has many opportunities for advancement, especially with monitoring, analysis, and modeling aspects of EarthMAP.

The existing data for constructing the model are multidisciplinary by necessity. This includes (in part) geology, surface water flows, water chemistry, groundwater levels, vegetation, land cover and use, snow cover, and water use data. Local scale and regional geology are used for defining soils and regional hydrogeologic units for the entire Upper Colorado River basin. The hydrogeologic units are defined in geologic framework models (already published as data releases) that define the spatial extent of permeable and impermeable units, as well as the connections between units. The watershed model is parameterized and calibrated with a wide range of vegetation, land use and land cover, snow cover, recharge, and surface hydrology data. Many of these data are derived from global and CONUS-wide satellite data products by NOAA and international hydrologic models (WaterGAP).

Many opportunities exist for new scientific advancement and capabilities through innovative data collection and monitoring and new modeling approaches. New collaborations with NWS and the National Center for Atmospheric Research (NCAR) aim to modernize the hydrologic modeling portfolio of the USGS. Innovative observation is the thrust of NGWOS, such as satellite-derived streamflow monitoring and inexpensive-but-widely-dispersed streamflow sensors across the landscape. Our model can immediately use new data types to resolve hydrologic dynamics, improve model calibration, assess model uncertainty, and implement data assimilation by directly combining model predictions with observations.

\section{Operational Implementation and Technical Considerations}

The expansion to an operational model can be done with existing models and computational infrastructure in the USGS but many aspects will require additional capacity expertise that might not be currently available in the USGS. The GSFLOW model will be complete and ready for transition to an operational framework in fiscal year 2022. This can be done by existing staff on the Colorado Plateaus project, by USGS developers of the software codes for GSFLOW and the code Precipitation-Runoff Modeling System (PRMS), and by staff on the former USGS project "WBEEP" (Water Budget Estimation and Evaluation Project; part of which is now contained in the National IWAAs Integrated Products project). The WBEEP project released an operational version of the National Hydrologic Model (NHM) in fiscal year 2020 that runs on the USGS supercomputer Denali. The NHM is based on the PRMS modeling code, the same code contained in GSFLOW for simulating watershed hydrology. It is feasible to implement existing tools from the WBEEP project to operationalize the GSFLOW model. The Colorado Plateaus GSFLOW model is already being run on Denali for calibration and evaluation.

Forecast input data from gridMET provides surface meteorological drivers. gridMET data are gridded datasets that are updated daily (data exist for prior day) at 4-km resolution for the entire CONUS. gridMET provides daily and seasonal forecast products from NOAA's Climate Forecast System (CFSv2), which uses four daily ensemble forecasts that span the next six months. Both the NHM and the Colorado Plateaus GSFLOW model are driven by gridMET data. The operational NHM model acquires data for the prior day's meteorological drivers (precipitation and temperature) and automatically runs the model daily. 
Additional tools and capabilities needed to operationalize the model include data assimilation, and data storage and mechanisms for the public and cooperators. Data assimilation, in addition to calibration, constrains model states and predictions on a real-time basis to improve model fidelity. Data assimilation is rarely used in USGS hydrologic models but fully implemented in weather forecast models. Expanded data delivery tools will be needed by cooperators and the public (for example, Pangeo, Google Earth Engine, USGS GeoDataPortal).

\section{Longevity and Sustainability}

This Use Case is likely to have many years or possibly decades of viability based on cooperator need and improved capacity and technology developed under EarthMAP. The issues of water availability under human and climatic stresses are likely to continue indefinitely. The need for this Use Case is demonstrated by the launch of new USGS Water Resources Mission Area priorities: new innovative observation networks, advancements in integrated water resource modeling and assessment, improved delivery of products to stakeholders, and modernization of our internet presence and product delivery. This Use Case addresses those priorities.

This Use Case outlines several advances in tools using existing USGS software and operational modifications that will continue to be in high demand and are likely to transfer to any hydrologic environment with integrated groundwater and surface water. The USGS and USGS Water Resources Mission Area aims to advance its hydrologic modeling software to new modeling paradigms of other agencies like NCAR. These paradigms include tight coupling of atmospheric and surface water models (for example, WRF-Hydro). However, tools that couple groundwater with atmospheric and land surface processes are not yet available; USGS GSFLOW is the state-of-the-art tool for integrated groundwater and surface water modeling. Ultimately, the longevity of this Use Case may be extended into the next decade through collaboration with NCAR by coupling with atmospheric models, and the authors of this Use Case are enthusiastic about this opportunity.

New observation networks by EarthMAP and NGWOS will be directly implemented in this Use Case. Extensive data networks will be needed to evaluate the performance of the operational model, assess forecast uncertainty, and to drive model predictions through data assimilation. There are many opportunities to collaborate directly with Reclamation, NWS, BLM, NCAR, and other agencies to develop performance metrics based on new and existing networks, and the forecasts are likely to improve through that engagement.

Primary Contacts: Jesse Dickinson, jdickins@usgs.gov; Melissa Masbruch,mmasbruch@usgs.gov

\section{Integrating Environmental and Economic Data and Modeling to Develop a Multi-Objective Resource Planning Tool}

Sustainable social, environmental, and economic resource planning requires balancing many competing interests. This use case will integrate environmental and economic data and modeling into an easy-to-use interactive mapper decision support tool that will allow users to identify outcomes and trade-offs of resource management decisions (fig. 5.10).

\section{Demand and Relevance}

Natural resource management depends on accurate and timely environmental modeling and data integration to support a broad range of environmental and societal water issues across spatial and temporal scales. These applications require continued and evolving advances in watershed, ecological, and economic modeling to address concerns related to water availability (quality and quantity) and environmental and social impacts and to provide timely information to scientists, managers, and policy makers. Further, pressing water availability challenges warrant new strategies for disseminating model results that effectively educate the public and aid resource managers and policy makers. This use case will inform decisions surrounding impacts climate change and water, land, and resource management strategies may have on water availability, and environmental and economic sustainability. The initial focus is on the Upper Colorado River Basin (UCRB), although the work is easily scalable to regional and national scales.

Stakeholders include states, tribes, other Federal agencies including the Bureau of Reclamation, Fish \& Wildlife, and the Bureau of Land Management. These stakeholders need information on the effects of environmental changes on a wide range of ecological and human systems such as water management including water rights, reservoir operations, hydroelectric power generation, managing wildlife habitat and water for ecosystems, allocating water for various uses and users. In the UCRB, these stakeholders have an immediate need for hydrologic information on the conditions that would triggering various elements of the Drought Continency Plan. The Salinity Control Forum is also an important stakeholder in the basin interested in information on how to manage water quality. Stakeholders also include other water users (agricultural, municipal, industrial, ecological) who use water for drinking, growing crops, and habitat. Information on water availability is important for these users to plan crops, recreation activities and businesses, and ecological habitat protection and restoration efforts. 


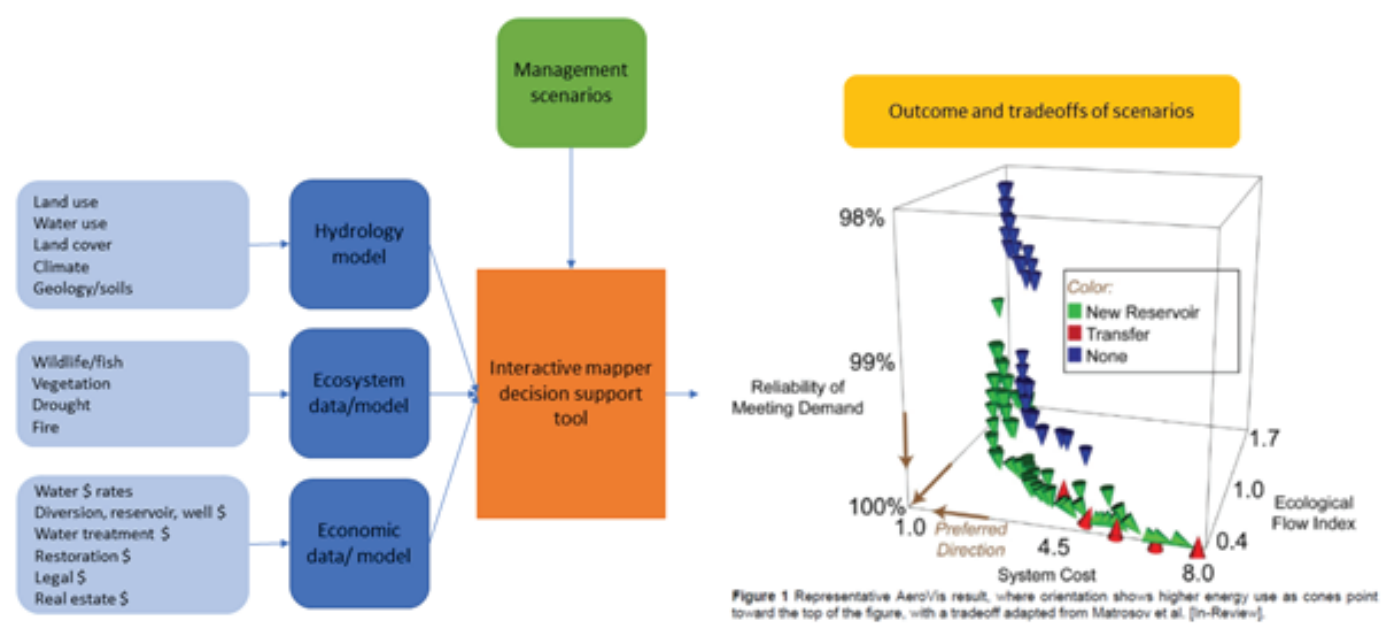

Figure 5.10. Diagram showing integration of environmental and economic data into models and testing of management scenarios using the interactive mapper decision support tool to evaluate effects and tradeoffs of different choices. For example, consider a case where a water manager is planning how to meet growing water demand with current water supply. They could test scenarios where a new reservoir is built, different amounts of agricultural land are transferred to urban land, and municipal use was increased by different amounts while agricultural water use decreased. They could then compare the effects of these changes on streamflow, water quality, vegetation, fish habitat, and the associated costs to identify a scenario where they could maximize the amount of demand met at the lowest cost while still maintaining sufficient ecological flows. The variations on these scenarios are represented by each point on the pareto-front, and the user can select the scenario that balances multiple objectives.

In its more developed stage, this use case will integrate data and models of streamflow and water quality, regional climate, water use, land cover, geology, ecology, and economics to illustrate the multifaceted effects of changes in climate, water use, land use and land cover on water availability for human and ecological needs. The results will be packaged in an online interactive mapper decision support tool that will allow users to understand the effects of resource management and planning decisions on water availability, quality, ecological impact, and cost.

This work directly supports DOI, USGS, and USGS Water Resources and Ecosystems Mission Areas priorities to provide scientific information about natural resources to address societal challenges, deliver integrated observations and predictions of the future state of natural systems at regional (or national scales), and achieve sustainable management and conservation of its natural resources.

\section{Improved Decision-Support}

This work will improve information scope and value and provide results faster and in a more accessible and useable format than currently exists. The improved decision-making ability will allow users to make decisions that consider a wide range of potentially conflicting objectives and understand the tradeoffs of decisions. They will be able to understand the effects of decisions on multiple entities (for example, cost, tradeoffs among opposing stakeholders, etc.) and effects of changes on hydrologic, ecological, and human systems. 


\section{Science Capabilities and Advancement Opportunities}

Existing data, science, models, and IT: Currently, long-term average models exist for streamflow, dissolved solids, total nitrogen, total phosphorous, suspended sediment. These are SPAtially Referenced Regression On Watershed attributes (SPARROW) models that cover the Southwestern U.S., including the UCRB. The most recent dissolved solids model is focused on the UCRB. There are also similar models covering the rest of the U.S. that would support national applications. SPARROW is a hybrid statistical and process-based model that estimates constituent loads in streams by linking monitoring data with information on watershed characteristics and contaminant sources, routed through a stream network. These models draw on a wide variety of existing spatial data including geology, topography, soils, land cover, land use, vegetation, human activity, climate, and water use to predict streamflow and water quality throughout the study area. Results of SPARROW models have been presented through online mappers (for example, the Southwest mapper). New developments in the RSPARROW code could also support interactive decision making (Alexander and Gorman Sanisaca, 2019). SPARROW is a useful tool for this use case because it easily accepts new data, has reduced computational costs relative to more physically-based models, is explicitly spatially referenced, has improved prediction accuracy over more physically-based models at larger scales, and is easily scalable to larger areas.

We are working on two existing USGS projects that this work aligns with. One project is applying projections of future climate and the surface water streamflow model to bracket the range of projected streamflow for the region. I am also working on a project to estimate historical time-varying salinity loads from irrigated agricultural lands throughout the UCRB using the UCRB dissolved solids model. Currently the model is a long-term average model, but it will be modified to represent temporal changes.

The USGS and other Federal agencies have developed a wide range of currently existing ecological information for the region that complements stream model results including data on wildlife, fish, invasive species, climate change refugia and adaptation science, and drought effects on ecosystems. The Bureau of Reclamation has extensive data and models to support reservoir management.

Current expertise in environmental economics exists within the USGS including the Social and Economic Analysis group in the Fort Collins Science Center, which could support incorporation of the social science and economic data and modeling aspects of the analysis.
Next steps-Modeling advancements: The next steps to developing this use case include linking the streamflow and water quality models so they could be run concurrently. This would support an integrated modeling approach to answer questions about how changes in land cover, land use, water use, climate, and other factors will jointly affect water quality and quantity. Results could then be integrated with information on regional ecology and economic data and models to understand the broader impacts of such changes on ecological and human systems.

Another advancement would be in convert long term average SPARRROW streamflow and water-quality models to work on an annual basis. This would provide more temporally-specific information and support understanding of current and future changes that may evolve with time.

Next steps-Development of an interactive mapper to support decision making: As a first step, the interactive mapper would allow the user to try out different scenarios and visualize results and quantify expected impacts. For example, the user could test how an increase in temperature would affect streamflow across the study are or in their area of interest. Depending on the modeling advancements, scenarios could become more complex. The user could download results and visualizations for their own uses.

A next step would be to integrate additional data to explore economic and ecological impacts of scenarios. The mapper would evolve into a more sophisticated decision support tool to help users to visualize and explore tradeoffs in scenario results using, for example, pareto sort techniques, a variety of plotting tools, and animations. The tool would facilitate exploring large multi-objective solution sets to aid in decision making, including the ability to optimize solutions based on stakeholder preference. In this way, the user can balance multiple conflicting goals and understand the tradeoffs associated with decisions. Such decision support tools will provide a clear idea of the optimal solutions for different combinations of objectives.

\section{Operational Implementation and Technical Considerations}

The USGS is well-positioned to initiate this Use Case and support its progression. We have extensive existing environmental data and models, and interactive mapper capabilities that support project initiation. The USGS Web Informatics and Mapping (WIM) team develops web-based tools using the latest technology to support data integration and visualization, including digital cartography, data management, data analysis, and decision support. This use case would likely require a cloud-hosting technology in order to make it easily accessible to internal and external users. 


\section{Longevity and Sustainability}

This use case could persist long-term, particularly given the anticipated high demand for information on water supply in the UCRB. In other regions, where questions of water quality or other environmental impacts may be more pressing, the use case will be relevant for answering different questions. Some of the driving input data would require updating as time progresses, science advances, and new understanding is developed, but the ability to test scenarios is real-time would allow the product to be persistently useful. Because many of the underlying data and models already exist for other geographies, the use case is easily transferrable and scalable.

Primary Contact: Olivia Miller, omiller@usgs.gov

\section{Fire Impact on Water Quality and Ecosystem Function in the Rocky Mountain Region}

This EarthMAP Use Case seeks has the objective of creating a spatial risk analysis of post-fire impacts on surface water quality and ecotoxicology of related to trace metals released from soil during wildfires.

\section{Demand and Relevance}

The objective of this case study is to determine surface water bodies, including lakes and streams, that are at greater risk for trace metal contamination during wildfires with a focus on impacts to avian waterfowl and fisheries. Stakeholders for this effort include the USDA Forest Service (USFS), the National Park System (NPS), U.S. Fish and Wildlife Service (USFWS), tribal fishery programs, state fish and wildlife agencies, and municipal water suppliers. Having a holistic approach to understanding the drivers of trace metal contamination post-fire can assist these entities as well as the USGS in developing attainable post-fire monitoring goals and help to interpret current fish and wildlife toxicology data. This case study proposes to synthesize remote sensing data for fire history with records of legacy mines, atmospheric deposition, soil chemistry, water chemistry, and animal tissue studies to find linkages between fire, trace metal mobilization and ecotoxicology.

\section{Improved Decision-Support}

Time and funding resources limit monitoring targets that are needed to understand ecological and human health impacts from fires. Process-level information gained from studies on a small watershed scale are critical in understanding the impact of fire on trace metal mobilization from legacy mine lands and other sources to fisheries and water supply. On the larger scale proposed under the EarthMAP program, an integrated approach can be applied to identify watersheds or ecoregions that are more sensitive to trace metal mobilization from legacy mine lands and soils that have received atmospheric deposition of mercury. Input from stakeholders and land managers within the study area, including USFS resource managers, USFWS fisheries and waterfowl managers, and NPS resource and research offices, should be sought to refine natural resource management targets that are the end-goal of larger scale data evaluations.

\section{Science Capabilities and Advancement Opportunities}

Mercury is volatilized from forest soils during fire (Webster and others, 2016) with trace metals from legacy mine lands transported into waterways through storm runoff following fires (Murphy and others, 2020). However, a direct linkage between fire and impacts to species that rely on these waterways needs to more effectively established. Extant satellite imagery including AVRIS coverage would be used as a regional framework to determine burn history. Other spatial data available include soil data from the USGS Geochemical Landscapes project (Smith and others, 2019), National Atmospheric Deposition Data for mercury, mercury bioaccumulation data from dragonfly larvae (Eagles-Smith and others, 2020), and baseline water chemistry collected through the National Water-Quality Assessment (NAWQA) program. The Mineral Resources Program USMIN project is synthesizing information on mine locations and commodities across the United States. All of these data are valuable, but synthesizing large datasets across watersheds to regions can yield new information on challenging, integrative tasks that inform land use management decisions following wildfires. 
Operational Implementation and Technical Considerations

Using a GIS platform to develop Expertise within the Geology, Geophysics, and Geochemistry Science Center (GGGSC) can carry out aspects of this work. Ray Kokaly (hyperspectral remote sensing) has worked with airborne visible/infrared imaging spectrometer (AVIRIS) datasets from prior fires, including the 1988 Yellowstone fires, the 2000 Cerro Grande Fire in New Mexico, and the 2009 Station Fire in Los Angeles County. Other information of fire extent and intensity can be provided by the Land Change Monitoring, Assessment, and Projection (LCMAP) developed in the Earth Resource Observation and Science (EROS) Center. JoAnn Holloway (biogeochemistry of trace metals) can develop the geographic extent of the proposed Use Case based on availability of adequate data coverage for fire history and burn intensity relevant to different NPS or USFS administrative units within the Rocky Mountain Region. The USMIN project led by Jeff Mauk and Carma San Juan in GGGSC has mine data coverage and GIS capacity to develop the spatial framework for these data. Maggie Goldman and Ben McGee work with visualization of data using a GIS platform, and Ben is also developing eDNA applications for detecting presence/ absence of fish species. Expertise from other USGS programs can round this effort out, including aqueous geochemistry (Blaine McCleskey and Sheila Murphy, Water Resources Mission Area-Boulder), aquatic invertebrate ecotoxicology (Travis Schmidt, Wyoming-Montana Water Science Center [WSC]; Johanna Kraus, Columbia Environmental Research Center), and vertebrate ecotoxicology (Collin Eagles-Smith, Forest and Rangeland Ecosystem Science Center [FRESC] Corvallis). David Krabbenhoft (Wisconsin WSC) is a leading expert on mercury contamination in aquatic ecosystems. Adding a component of economic loss of water quality from fire impacts through the Water Economics group at the Fort Collins Science Center [FCSC] can add an increased societal impact.

This effort does not replace USGS science in areas burned by wildfires. A regional EarthMAP effort can be augmented with smaller-scale studies in the USGS and by developing cooperative relationships with local, state, and federal land use managers.

A paleofire approach (Natalie Kehrwald, Geosciences and Environmental Change Science Center [GECSC]) to understand wildfire occurrences from the perspective of Holocene climate change in northern New Mexico can be augmented with data synthesis from the Cerro Grande fire. New samples can be collected in coordination with local water resource agencies for mercury, lead and other targeted metals in lakes that are sources for municipal and rural water supplies.

An ongoing survey in the Yellowstone National Park Research Office of loon biometrics, including lead and mercury blood chemistry can be augmented by a synoptic sampling of lake chemistry associated with the bird populations, with background information on historical fire intensity derived from AVIRIS data. Erin White, Park Hydrologist at Yellowstone National Park (YNP) has indicated an interest in coordinating YNP research interests with USGS work in relationship to a long-term monitoring of the common loon by YNP avian biologists. Environmental stressors for the common loon includes mercury and lead, which have been found in elevated concentration in YNP populations. Little work has been done of post-fire resource impacts in Yellowstone since the 1988 fire that burned 793,880 acres in the park. The capacity for lakes to act as long-term sinks for mercury released through wildfire and the availability of an immediate partnership with the YNP research office, and the public visibility of study outcomes would make the greater YNP ecosystem a good testing ground for an EarthMAP approach to interrogating a complex regional problem with a multidiscipline approach.

Increased data storage and computational resources will be needed for the synthesis of geospatial datasets, particularly at the scale of the Rocky Mountain Region. In the short term, it may be a more attainable goal to form partnership with different NPS and USFS units.

\section{Longevity and Sustainability}

The changing climate regime for the western United States is yielding more persistent drought conditions, increased frequency of fires, and a longer fire season. Providing data synthesis approaches to understanding areas more at risk for water quality and ecosystem impacts from trace metal transport will provide a durable input for prioritizing monitoring sites and post-fire mitigation of risks to human and environmental health. This Use Case proposes to develop approaches to understanding geographic patterns of risk associated with wildfire. The approach can be scaled up to understand regional impacts or scaled down to interrogate process-level trace metal mobilization on a watershed scale.

Primary Contact: JoAnn Holloway, jholloway@usgs.gov

\section{Predicting Future Water-Quality Changes in Mineralized Headwater Catchments in the Upper Colorado River Basin}

This Use Case involves developing an empirical GIS-based modeling tool that could determine the likelihood of future water-quality degradation in mineralized mountain headwater catchments within the Upper Colorado River Basin (UCRB) due to climate warming. A predictive tool of this type would be valuable to state and federal agencies who manage surface water resources, fish populations, and abandoned mine site cleanup efforts. 


\section{Demand and Relevance}

Numerous studies have observed increasing solute concentrations in mountain headwater catchments around the world that appear to be due to warming temperatures and shifting precipitation regimes caused by global climate change. Watersheds containing abundant sulfides (mineralized watersheds) may be particularly susceptible to these changes because of the sensitivity of sulfide weathering to subsurface oxygen transport and redox conditions, which are directly linked to climate-controlled factors such as groundwater recharge rate, water table depth, and extent of frozen ground. This susceptibility raises a significant concern regarding potential increases in future stream metal loads in mineralized headwaters, which could negatively impact stream ecosystems and downstream water resources. Mine tunnels and exposed waste piles from historical mining activities could further exacerbate the problem, as acid drainage from these features could be yet more sensitive to climate variability than natural background acid-rock drainage. The UCRB has many mountain headwater catchments containing extensive mineralization and legacy mining features. A possible Use Case for EarthMAP is therefore the development of a regional-scale modelling tool for predicting future water-quality changes in mineralized headwater catchments in the UCRB that could be useful to state and federal agencies who manage water resources, fish populations, and abandoned mine site cleanup efforts.

Two projects cooperatively funded by the USGS Mineral Resources Program and the Department of Energy's Subsurface Biogeochemical Research Program are currently underway that focus on water-quality issues in mineralized headwater catchments in Colorado. This work is being performed collaboratively with scientists in the Colorado Water Science Center, with additional support from the USGS Toxics Program. The first project focuses on improving understanding of processes controlling acid-rock drainage generation and metal transport in mineralized mountain watersheds, and the sensitivity of these to climate warming. The second focuses on developing science-based approaches for screening legacy mine sites to aid in the process of prioritizing sites for remediation work. These projects have high stakeholder interest, involving active engagement with representatives of the USEPA Region 8, Colorado Division of
Reclamation, Mining, and Safety, and with Trout Unlimited. We fully anticipate that the Use Case work proposed below will have the same high level of stakeholder interest, will be performed mostly by the same group of GGGSC and Colorado WSC scientists working on these current projects, and will also be considered priority work by the Mineral Resources and Toxics Programs.

\section{Improved Decision Support}

A regional-scale modelling tool capable of indicating the likelihood of future water-quality degradation in different mineralized headwater catchments in the UCRB could be particularly beneficial for three groups of agencies. The first group includes agencies tasked with managing stream fisheries which are vital for tourism, primarily the U.S. Fish and Wildlife Service and, in Colorado, the Colorado Parks and Wildlife Department. Knowledge of which stream networks are more or less threatened by future increases in metal loads could help considerably in decisions regarding where to focus stocking and habitat improvement efforts to develop the most robust and sustainable populations. The second group includes agencies tasked with managing abandoned mine site cleanup efforts, primarily the USEPA and, in Colorado, the Colorado Division of Reclamation, Mining, and Safety. Knowledge of which mining-impacted watersheds may experience the most pronounced additional water-quality degradation in the future would be highly valuable in the process of deciding where to invest new or enhanced site clean-up efforts to maximize benefit to the environment. Furthermore, an awareness of the potential for increasing natural background metal concentration in watersheds where remedial work is underway is important in the process of establishing attainable clean-up standards, and developing plans for adaptable standards at sites where background concentrations are likely to be a moving target. The third group is municipal water resources managers in mountain and mountain-adjacent communities, where surface water is often the primary source for public supply. Knowledge of which catchments in their source stream networks may experience water-quality degradation, and to what degree, would help water managers anticipate future required modifications to water intake locations and treatment systems, and allow them to plan accordingly. 


\section{Science Capabilities and Advancement Opportunities}

Two fundamentally different types of models could potentially be developed for the purpose of predicting changes in surface water quality. The first is a regional-scale numerical reactive solute transport model, but these complex process-based models require extensive parameter inputs and computational power that will likely limit their application beyond the small-watershed-scale for many years to come. The second is a much simpler empirical model that would utilize a set of observed correlations between mapped watershed physical characteristics and measured stream water quality changes (disregarding the 'why') to predict the latter when only the former is known. To our knowledge, a regional-scale empirical model for this application has not been developed and is thus aspirational. However, we believe that our current understanding of the factors controlling sulfide weathering, available map coverages, and available statistical tools for deriving necessary correlations are all sufficient to conceivably develop an empirical model in a few years.

We envision the following general approach for developing such an empirical model. Central Colorado could be used as a model 'training ground' given that a GIS model has already been built for the Central Colorado Assessment and Legacy Mine Lands projects (CCAP and LML), containing most of the required data coverages (for example, bedrock lithology, hydrothermal alteration mineral mapping from hyperspectral data, and mine features from USMIN). A comprehensive sampling program for stream water chemistry would be undertaken throughout the region, perhaps in cooperation with the Colorado WSC. Targets would include all mineralized watersheds where historical water chemistry data exist, including the numerous sites sampled $\sim 15$ years ago for CCAP. Changes in metal concentrations over recent years could then be assessed and compared to watershed physical features to identify correlations using principle component analysis and other advanced statistical tools. Artificial intelligence and machine learning could also be very useful for this purpose. The next phase of the work would involve expanding the GIS model to other parts of the UCRB where historical stream water-quality data are available, collecting new data from these sites, and testing the model's capability to predict observed changes. Finally, if the model passes these tests, it could be expanded to the entire UCRB. Regarding partnerships with stakeholder agencies, these are already well established through our current project work and could be directly utilized to in the proposed Use Case for obtaining stakeholder input and adapting the model tool to best meet stakeholder needs.

It should be understood that success for this effort is admittedly uncertain, with it possibly yielding insufficiently robust correlations to enable development of an empirical model. However, the water chemistry data alone would still be highly valuable to establish regional baselines and trends, and to assess the regional extent of recent surface water-quality deterioration in mineralized watersheds to gauge the scale of the potential problem. Furthermore, the cost of this effort would be relatively low, heavily leveraging prior CCAP and LML project work and requiring only the collection of standard water chemistry data and application of standard GIS and statistical modeling tools. Regardless, this approach clearly presents the most tractable path forward if the development of a predictive capability for water quality on the scale of the UCRB is determined to be a priority for EarthMAP.

\section{Operational Implementation and Technical Considerations}

The research team from the GGGSC and Colorado WSC working on the two current USGS projects described above possesses most of the scientific capacity needed to implement the proposed Use Case. One key gap is the need for additional expertise with applying statistical analysis to GIS models, particularly if developing correlations is more challenging than anticipated and demands the application of AI/ML. Another operational need is additional staff to collect the extensive set of surface water-quality samples throughout Central Colorado, though, as noted above, this short-term need could possibly be met by the Colorado WSC, or perhaps temporary student hires. As far as the utilization of the empirical model-based tool by stakeholders once developed, we envision that this would require only minor initial training because it would function on a GIS platform with which stakeholders already have considerable experience. 


\section{Longevity and Sustainability}

The proposed Use Case should have considerable long-term relevance because climate prediction models consistently indicate that the climate will continue to warm through the 21 st century, meaning that water quality degradation in mineralized watersheds poses an ongoing challenge that could persist for many decades. The empirical water-quality prediction tool we propose should thus also be applicable and useful for many decades. However, it would certainly be yet more useful if it were a living model that is updated and improved through time. A monitoring program involving ongoing collection of surface water chemistry samples from a select set of sites would enable continued testing of the model's accuracy, and the statistical exercise of deriving correlations between observed water-quality changes and watershed characteristics could be relatively easily re-run periodically to refine and improve these correlations. In this way, the model would likely become ever more accurate and useful through time. The transferability of the UCRB-specific empirical model we develop to other regions is uncertain, presumably limited by the degree to which the other regions differ climatically and geologically from the UCRB. However, the process of empirical model development we propose should be entirely transferable to other regions and enable the creation of other region-specific empirical models for other mountainous areas of the U.S.

Primary Contact: Andrew Manning, amanning@usgs.gov

\section{Energy Resource Development Potential and Tradeoffs, to Support Integrated Analysis for "All of the Above" Energy Strategies}

Decision makers considering future impacts of fossil fuel and renewable energy development on land, water and socio-ecological resources often desire information on where future development is most likely to occur, in addition to estimates of resource quantity or quality. The USGS can address this stakeholder demand by developing approaches in collaboration with other science agencies for estimating future energy "development potential," and for projecting impacts of development to other resources within areas of interest, by synthesizing diverse data and analysis.

\section{Demand and Relevance}

Traditional USGS oil and gas assessments provide estimates of the total undiscovered technically recoverable petroleum resource in a given region. While that information that is used by a wide range of decision makers in the public and private sectors, increasingly those stakeholders are also interested in more specific information regarding which areas within a given study region have greater potential for oil and gas development. Interested stakeholders include entities responsible for resource management such as state and federal land and wildlife managers, and individuals and groups with varied interests in resource use such as recreational interests, private property owners, and wildlife advocates. In addition, increasing emphasis in much of the western U.S. is focused on other energy resources, including geothermal energy and renewable resources such as wind and solar power, presenting a similar set of questions, as well as the potential complexity of conflict or synergy between energy resource development and existing uses and benefits provided by the landscape.

Accurately predicting energy resource development activity in space and in time is not a realistic goal, as actual energy industry activities depend on a wide array of economic, regulatory, and social factors in addition to geologic, environmental, and a suite of logistical considerations. However, it is possible to develop an approach that identifies locations where energy development is more likely based on a set of predictor variables, in order to address stakeholder questions on resource development potential and desirability. The task of addressing this stakeholder need will require synthesis of energy resource information (geologic and petroleum-related, as well as solar and wind potential), along with consideration of existing uses, ecological attributes, terrain, roadways, infrastructure, and associated factors. The result will not be a prediction of specific development that will occur, but rather a set of mapped variables that can be used to estimate the relative potential and suitability for resource development. This information will be useful to inform decisions regarding the optimal management of public lands.

The development of a decision support tool that addresses energy development potential will draw on the science products and expertise from multiple USGS programs and Mission Areas and collaborations with the National Renewable Energy Laboratory. Though the participating science centers are largely based in the USGS Rocky Mountain Region, the topic of interest is national in scope and potentially involves all USGS regions. The demand for this sort of decision support tool is long-standing, and interest in these products will likely increase as land-use decisions and energy resource concerns continue to gain prominence. Development of USGS products to address these needs will be a long-term activity, with the near-term objective of addressing immediate stakeholder interest, followed by opportunity to hone these products and improve their applicability to stakeholder decision-making needs. 


\section{Decision Support}

Due to the challenges of estimating energy resource development potential in a meaningful manner, current tools are limited, and no USGS science directly addresses this question. As such, the development of a scientifically based decision-support tool that delivers information on development potential, including co-located resources and associated tradeoffs, will be an improvement. By synthesizing relevant science and information, the USGS can provide "actionable intelligence" that stakeholders can confidently rely on for use in their planning and decision making. Because the USGS is the recognized authority on many of the relevant scientific components, we are the logical organization to synthesize this information; we are best equipped to understand both the capabilities and the limitations of these data.

The myriad factors that influence energy resource development patterns, and the complex interplay between them, dictates that a decision-support tool will only be useful if developed through close interaction with stakeholders to ensure that their needs are being addressed, on timeframes that are useful to them, and to effectively communicate both capabilities and limitations of the analyses. Through interaction and communication between scientists and decision makers, we can ensure that stakeholder desires are met and that the decision support product honors scientific limitations.

\section{Science Capabilities and Advancement Opportunities}

The objective of this work is to address stakeholder questions regarding development potential for energy resources of all kinds, with initial focus on oil, gas, solar, and wind. The work draws on data and expertise from across much of the USGS, and from outside sources.

The USGS Energy Resources Program (ERP) produces oil and gas resource assessments that include a wealth of geologic and petroleum related data and analysis in addition to the assessment results themselves. This information includes geologic maps and cross sections, petroleum production data, and expert analysis of these data. In addition, the ERP also studies patterns of water use and water production, yielding another set of data for decision-support questions where water-related topics are relevant. Together, this information provides a strong foundation for the petroleum-related parts of the synthesis work.

For solar and wind energy, USGS will collaborate with the National Renewable Energy Laboratory (NREL) to better parameterize their existing energy development tools. NREL has developed two models to forecast energy development. First, Regional Energy Deployment System (ReEDS) forecasts national energy demand and the "mix" of energy generation meeting this demand across a wide array of future scenarios. Second, for solar and wind energy generation, the Renewable Energy Potential (reV) Model, includes wind and solar resource maps, localized costs of energy generation, system performance as estimated capacity factors, and spatial exclusions based on socio-political and land use constraints, to estimate the amount of annual technical potential $\left(\mathrm{MWh} / \mathrm{km}^{2}\right)$ for wind turbines, photovoltaic solar, and concentrated solar production. USGS will contribute refined land-use and land-cover information, and improved information on environmental constraints (for example, sage grouse core areas), to allow more refined predictions of renewable energy potential in specific regions. In the long term, the spatial constraint algorithms in reV could be modified and applied in USGS Assessments Units when considering oil and gas development potential.

Predicting energy resource development potential further requires information on the social-ecological system(s) in which it will be embedded. Factors such as water source and use areas, key wildlife habitat areas, protected areas, transmission infrastructure, and established socio-economic uses such as recreation, aesthetic enjoyment, water-quality regulation, and others are all key to establishing the existing use of a landscape and hence the energy resource development potential. A variety of USGS programs have expertise in social-ecological systems, including Land Change Science, Science Decisions Center, Energy and Wildlife, Water Availability and Use, and Social \& Economic Analysis Branch. These groups will work together to characterize the social and ecological dimensions that must be weighed against resource potential to identify the tradeoffs and evaluate development potential. Stakeholder input will be used to establish the weighting schemes.

\begin{tabular}{|l|l|l|}
\hline \multicolumn{1}{|c|}{ USGS Mission Area/Other } & \multicolumn{1}{c|}{ USGS Programs } & \multicolumn{1}{c|}{ Role } \\
\hline \multirow{2}{*}{ Energy and Minerals } & Energy Resources & Petroleum resource assessment \\
\cline { 2 - 3 } & Science Decisions Center & Decision analysis, economics \\
\hline \multirow{2}{*}{ Ecosystems } & Land Change Science & Social-ecological systems \\
\cline { 2 - 3 } & Energy \& Wildlife & Biological resources \\
\cline { 2 - 3 } & Social \& Economic Analysis Branch & Social science \\
\hline Water Resources & Water Availability \& Use & Water resources \\
\hline Core Science Systems & Science Analytics \& Synthesis & Data delivery and decision support \\
\hline National Renewable Energy Laboratory & N/A & Solar and wind build-out models \\
\hline
\end{tabular}


Synthesizing and integrating the data described above in a meaningful manner represents a largely new scientific direction for the USGS, and it expands the kinds of contributions EarthMAP can make to those who rely on USGS for decision-relevant science. It will require development of new concepts for linking data and new ways to communicate possible future energy development and its consequences to stakeholders. It will also include development, through discussion with decision-makers, of definitions for "resource development potential," to address their particular concerns and interests. For example, some decision makers might want to know which areas are most favorable for oil, gas and wind power development based on wind, geologic and infrastructure considerations, whereas other decision makers in other areas might be interested to consider areas most favorable for gas and (or) solar development while minimizing impact to particular species of interest and (or) existing uses of public land. We envision a decision-support tool that has many "knobs" that can be used to adjust relative weighting of different map data, a tool that is mainly useful through thorough communication between scientists and decision makers.

\section{Operational Implementation and Technical Considerations}

This Use Case draws on a wealth of existing USGS and external information, but at the same time represents a new science direction. Determining the variables most important for resource development potential, and the relationships between them, will build from existing capabilities within the USGS and will require contributions from scientists in several USGS programs as described above. Specifically, the development of synthesis approaches for these diverse data, to provide a decision-support tool that is adjustable to the needs of a range of foreseeable decisions, will be an exciting new challenge.

Complementing USGS expertise, there will likely be a need for input on a few additional aspects of the work. For example, colleagues at the National Renewable Energy Laboratory are well on their way to an EarthMAP-like synthesis framework for renewables but need USGS expertise in wildlife ecology and land change science to better inform their geospatial constraints models. Regarding oil and gas development, we will need experts who can provide guidance regarding logistical aspects of oil and gas development, including drilling engineering and transmission/ transportation details.

Effective delivery of the resulting decision-support tool, and handling of the underlying data, will most likely be possible using existing web-based tools, possibly with need for some expansion of existing capabilities. It is not anticipated that the tool will require high computational power
(NRELs REEDS/reVs runs on their supercomputer), but data synthesis and effective delivery is an important component of this work.

\section{Longevity and Sustainability}

Energy resource development has long been a topic of interest for land management agencies and this is unlikely to change in the foreseeable future. Emphasis on particular energy resource types may vary over time, however, and this is a reason to include not just oil and gas but also solar and wind development potential in this Use Case. The need for greater information on resource development tradeoffs is ongoing both during planning stages of energy resource development and throughout the development process.

The decision-support tool that we describe herein is not limited to any particular geographic area, and its utility is anticipated to be essentially nation-wide (any area with energy resource development potential). By nature, a tradeoff exists with scale, where greater spatial specificity comes with greater uncertainty; that is, when applied at larger scales this decision support tool can be anticipated to provide more meaningful results. Expansion of the developed approaches to include additional energy resource types is feasible, though each resource type will require additional considerations related to the particular geologic, ecological, social, and other factors that control resource development likelihood and associated tradeoffs.

Primary Contact: Seth Haines, shaines@usgs.gov; Darius Semmens, dsemmens@usgs.gov; Jay Diffendorfer, jediffendorfer@usgs.gov; Karen Jenni, kjenni@usgs.gov

\section{Resource Assessment Platform}

This Use Case envisions providing decision makers with a centralized platform for various data and analytical tools needed for comprehensive resource management. A pilot in the Colorado River Basin is proposed, with the objective of prioritizing investments, optimizing efficiencies, and minimizing costs for Federal resource-management activities in the Basin.

\section{Demand and Relevance}

Stakeholders require data and tools to characterize and assess effects of past and current resource development activities at a variety of spatial and temporal scales, and to address future effects. Quantitative forecasts and predictions of supply, demand, and effects of development on resources are needed and used by stakeholders to evaluate resource availability, develop land and resource management plans, and assess environmental and economic effects of resource development. 
This Use Case will inform decisions such as determination of the environmental impacts of Federal agency actions under the National Environmental Policy Act (NEPA); Federal, Tribal, and State decisions regarding recovery and management of threatened and endangered species under the Endangered Species Act (ESA) and related state laws and regulations; Federal, Tribal, and State management of land, forestry, and energy resources, among others. Relevant decision-makers, stakeholders, and intended information recipients; their interests in and need for improved science information. The pilot Resource Assessment Platform (RAP) would have immediate application to sister agencies of the USGS, and a fully developed version could be of use to stakeholders at many levels including:

- USEPA - environmental protection and effects of resource development/use

- USACE - water supply, water operations, wetlands management

- Bureau of Reclamation (BOR) — water supply, water operations, development of water resources

- Land and resource managers (USFS, USFWS, BLM, NPS, Tribes, State land agencies)—resource occurrence and availability, socio-economic and environmental effects of resource development and land management scenarios, protection/conservation of other resources (endangered species, cultural/ historical, etc).

- Resource users (Municipalities, private entities, etc.) forecasting resource supplies.

Information synthesis necessary to provide actionable resource management intelligence requires seamlessly integrating existing data and models. Tools would be developed to allow users to synthesize and deliver those data of interest to them at speeds appropriate for timely decision making. Pilot-scale implementation of this Use Case in the Colorado River Basin would be related to and supported by current USGS initiatives including implementation of the USGS Water Resources Mission Area's Next Generation Water Observing Systems (NGWOS) and Integrated Water Availability Assessments (IWAAs) priorities. Several Department of Interior (DOI) agencies have priority initiatives in the Basin, so the pilot would also be designed to support DOI agency activities. Once demonstrated at the pilot scale, the RAP could be extended geographically to other regions or the nation, updated periodically or in real time with updated input data and machine learning, and expanded to support additional resource management decision-making needs.

\section{Improved Decision-Support}

The RAP will provide stakeholders a single location to support evaluation of various decisions such as prioritizing remediation activities and assessment of future development needs and potential impacts quickly and confidently knowing that the information and tools are the most current available.

The ability of stakeholders to evaluate multiple resources simultaneously will be improved, so that decisions will better reflect integrated conditions and impacts. Availability of a comprehensive, central source of data and tools sufficient for a wide variety of resource assessments will improve timeliness of decision delivery. Opportunities to work directly with decision-makers to define their integrated science needs and understand and meet their needs for science delivery. Scientists will need to work closely with decision-makers to better understand assessments of interest. This ongoing, direct interaction with decision-makers will enhance the usefulness of the platform and ensure it remains relevant as partner needs and priorities evolve. Expected impact of the improved decision or resulting management-action. Because this platform can be modified to focus on variables of interest, stakeholders will better be able to make decisions based on the variables of interest to them. This will have the benefit of reducing the duration and costs of resource assessments, improving timeliness and enhancing the consistency of management decision making.

\section{Science Capabilities and Advancement Opportunities}

This project would take advantage of existing USGS scientific and technological capacity in water resources, climate, land cover and land use, geology, mineral and energy resources, species and ecosystems, modeling and parameter estimation expertise, the Advanced Computing Cooperative, 3 -DEP, and other capacities. A wide variety of existing scientific, modeling, remote sensing, and computational capacities, both internal and external, could also support the project. This platform would require collaboration between USGS water, ecology, geology, mineral and energy, and social science centers sharing data and expertise. The Colorado River Basin pilot application would be integrated with ongoing USGS NGWOS/IWAAs efforts, and leverage previous work under the National Streamflow Information Program (NSIP), Regional Aquifer-System Analysis Program (RASA), NAWQA and FAS. 
For the initial pilot application existing USGS datasets include: National Water Information System (NWIS), Simplified Surface Energy Balance (SSEBOP), EPA's STORET, USGS National Cooperative Geologic Mapping Program, USGS Geolog Locator, Weather datasets-PRISM and DayMet, USGS National Land Cover Database, LANDFIRE, USDA STATSGO, USDA-Natural Resources Conservation Service (NRCS) SNOTEL (SNOwpack TELemetry), USGS 3D Elevation Program (3DEP), etc. Numerous partner datasets and models also exist and are available for synthesis. Existing and potential partners include: other USGS Science Centers, Federal agencies such as BOR, BLM, NPS, USFS, USFWS, USACE, State land, water, forest, energy and other natural resource management agencies, universities, local and municipal users of resources, and other stakeholders such as nongovernmental organizations.

\section{Operational Implementation and Technical Considerations}

The data and modeling platforms needed for many resource assessments already exist, both within USGS and with external partners. The challenge would be linking these tools and providing the interactive platform and delivery mechanisms for decision makers. The approach would be a cloud-based interactive dashboard or integrated system of data layers and modeling capabilities. Something like Tableau might be useable or adaptable to meet the objectives of this Use Case, or a new type of platform may need to be developed. Agreements would have to be reached among the agencies to enable their legacy data to be easily pulled into the RAP for use and analysis. To be useful to decision-makers the RAP would need to be reliable, easy to use, with well-documented data lineage and interactive data-delivery mechanisms.

Partnerships with IT experts and programmers in the Advanced Computing Cooperative, the Center for Integrated Data Analytics, and the Oklahoma-Texas WSC would be needed. For the Colorado River Basin DOI pilot, close collaboration with the other DOI agencies will of course be essential. This Use Case will require high-level IT and related expertise to develop, deliver and maintain the RAP, including programming expertise and data scientists. Artificial intelligence and machine learning technology and expertise may be required to create a platform that improves functionality through use.

\section{Longevity and Sustainability}

Once the initial framework is established, longevity and sustainability are maintained by a constant process of incorporating newly available data sources, and expanding the ways in which the data sources can be synthesized and interrogated to address emerging needs. The relevance of this Use Case will expand over time with the addition of newly available datasets and tools, and the organic adaptation of the framework and capabilities to address emerging needs. Once demonstrated at a pilot scale, the geographic and administrative scope could be expanded to cover additional geographic areas and decision-making needs. The usefulness of the RAP as a decision support toolbox will be enhanced by support from persons capable of adapting the framework to support emerging stakeholder needs as they become identified.

Successful development of the RAP will be enhanced by limiting the initial scope to a regional pilot scale. The ultimate vision is to develop an interconnected web of regional tools that allow interrogation across and between regions, ultimately scaling up to handle national level questions. Information that will be used to evaluate the effectiveness of this effort and define metrics of performance and success. Effectiveness of the effort will be evaluated by the ability of the RAP to deliver information in a way that addresses stakeholder needs, and that supports timely decision making on a variety of issues. Other problems, at different scales, different timeframes, and or in different geographies, will benefit from the science and technologies developed for this Use Case. Expanding the scope beyond the regional pilot scale will expand the ability of the RAP to address problems at expanding scales and differing geographies. Incorporation of longer timeseries datasets and $\mathrm{AI} /$ machine learning will allow questions to be asked at a point scale, delineate past trends, forecast into the future, and expand RAP functionality.

Primary Contact: Michael Johnson, msjohnson@usgs.gov .Anne Tillery, atillery@usgs.gov

\section{Southeast Region}

\section{Alabama Barrier Island Restoration Assessment}

The Alabama Barrier Island Restoration Assessment (ALBIRA) Project was a science-based, collaborative effort between the State of Alabama, the U.S. Geological Survey (USGS), and the U.S. Army Corps of Engineers (USACE) to investigate viable options for the restoration of Dauphin Island, Alabama that can increase island sustainability and restore vital habitats for species affected by the 2020 Deepwater Horizon (DWH) oil spill. Completed in 2020, the study was funded through a grant from the National Fish and Wildlife Foundation Gulf Environmental Benefit Fund and study results are intended to inform future restoration decisions. 


\section{Demand and Relevance}

Hurricane Katrina in 2005 and the Deepwater Horizon oil spill in 2010 are two major events that have affected habitats and natural resources on Dauphin Island, Alabama. The latter event provided hundreds of millions of dollars to Alabama for coastal restoration and prompted a collaborative effort between the USGS's Coastal and Marine Hazards and Resource Program (CMHRP) and Ecosystem and USGS Water Resources Mission Areas, the USACE, and the State of Alabama, funded by the National Fish and Wildlife Foundation, to investigate viable, sustainable restoration measures that reduce degradation and enhance the natural resources of Dauphin Island. The overarching goal of the ALBIRA was to document present conditions and forecast potential conditions under varying sea-level change and storm scenarios for a no-action alternative and for a variety of restoration measures including beach and dune restoration, marsh and back-barrier restoration, and placement of sand in the littoral zone. Scoping sessions with the public and expert elicitation engagement from academia, state and federal partners, and nongovernmental organizations helped communicate climate-related threats to Dauphin Island and identify shared objectives that maximize the sustainability of the system. The integrated science and synthesis components incorporated into the project to address the goals and objectives include: (1) conceptual ecological model development; (2) system-level data acquisitions; (3) integrated hydrodynamic, geomorphic, water quality, and habitat modeling on decadal timescales; (4) evaluation of restoration alternatives using structured decision making; (5) monitoring and adaptive management plan development; and (6) online data catalog and delivery using a web map.

Although this study was externally funded, this effort aligns with the priorities of the USGS Mission Areas and programs discussed above. For example, the study aligned with the Ecosystem Mission Area's Land Change Science Program's main goal of developing a comprehensive understanding essential for managers and policy makers on topics such as: (1) rates, causes, and impacts of sea-level rise; (2) changes in distribution of plant and animal communities; and (3) causes and consequences of land-cover change. Additionally, the effort aligned with the USGS CMHRP Level 2 requirements: (CM2.3) “...provide actionable information and reliable guidance on coastal change hazards processes and impacts and, in alignment with the USGS Risk plan, identify and communicate the vulnerability to potential hazards in relation to user needs"; and (CM3.3) “... provide integrated models and forecasts of impacts of episodic and extreme coastal processes, climate change, human disturbance and restoration activities on estuaries, wetlands and reefs."

The models developed for this project can be extended to predict impacts across different time scales and different management applications. This could include understanding impacts from a contemporary storm through hindcast analysis (for example, understanding the vulnerability of a barrier island and its habitats to coastal change), real-time forecasting (for example, predicting where overwash or breaching may occur, which could inform emergency management decision making such as road access), and post-storm recovery assessments (for example, identifying changes in sand movement and the ability of a barrier island and its habitats to recover to a pre-storm state). In addition to the management actions considered in this project, actions such as creating vegetation features or implementing living shorelines could also be assessed. The science and products developed from this effort are scalable to other barrier islands with local-level calibration, as well as broader areas to provide a more regional picture of coastal impacts. This is currently being accomplished through synergistic USGS projects focused on nearby regions in the northern Gulf of Mexico.

\section{Improved Decision-Support}

Restoration measures for this effort were developed to address multiple objectives related to the social and ecological concerns of stakeholders on Dauphin Island and surrounding areas. The stakeholder defined objectives were to: (1) maximize ecological function and physical processes (that is, sustainability); (2) minimize social impacts and costs; (3) maximize coastal and marine resources; and (4) minimize time that it would take for a restoration action to provide benefits for the island. In all, 23 restoration and land acquisition measures were developed and assessed relative to their ability to meet these objectives on Dauphin Island. Bayesian Belief Networks - probability-based decision tools - were used to evaluate restoration measures that best met these competing objectives. This objective-centered approach to decision making explicitly incorporated the human dimension to help assess trade-offs among restoration measures. Working with our decision-makers from Alabama, a storymap was developed to communicate the results of the restoration assessment to the citizens and other stakeholders of Dauphin Island. The State of Alabama used the findings of our assessment to target over $\$ 150$ million worth of restoration measures for implementation. 


\section{Science Capabilities and Advancement Opportunities}

This project relied on existing USGS expertise in field data collection, predictive coastal morphological modeling, habitat mapping and modeling, and decision analysis to address management decision making and stakeholder needs at Dauphin Island (fig. 5.11). Guided by a conceptual ecosystem model developed for Dauphin Island for this study, field data were collected to gain an understanding of the island's historical evolution as well as the physical, topographic, bathymetric, geologic, and oceanographic setting to help maximize the likelihood of restoration success. These factors have not only played an important role in understanding how the island has evolved over time but will regulate how it will respond in the future. Field data collected during this study included: (1) bathymetric and geologic surveys; (2) wave and current measurements; (3) water-quality data; (4) sediment distribution information; and (5) habitat data. This information was used to update baseline conditions and provide a primary source of data for model development and validation. Forecasting barrier island evolution provides decision-makers the ability to assess the resiliency of coastal environments to future climate change conditions. The suite of numerical models developed for Dauphin Island provided a quantitative understanding of the physical processes governing the past and present Dauphin Island barrier system, including the nearshore region adjacent to the barrier island complex. Morphologic models were used to describe barrier island evolution accounting for fair-weather conditions, storms, and post-storm recovery. The models were used to assess potential future states of the island considering changes in sea level and storm variability (frequency and intensity of tropical cyclones) over a decadal period. Habitats were predicted using a model that leveraged landscape position linkages (for example, proximity to shoreline, elevation, and relative topography) for barrier island habitats. A spatially explicit habitat suitability model was developed for nearby estuarine waters for oyster and seagrass habitats. Together, the predictive morphological and habitat models were used to assess the previously mentioned management actions developed by stakeholders through structured decision making to gauge their ability to meet multiple objectives for habitats, sustainability, residents, and conservation values.

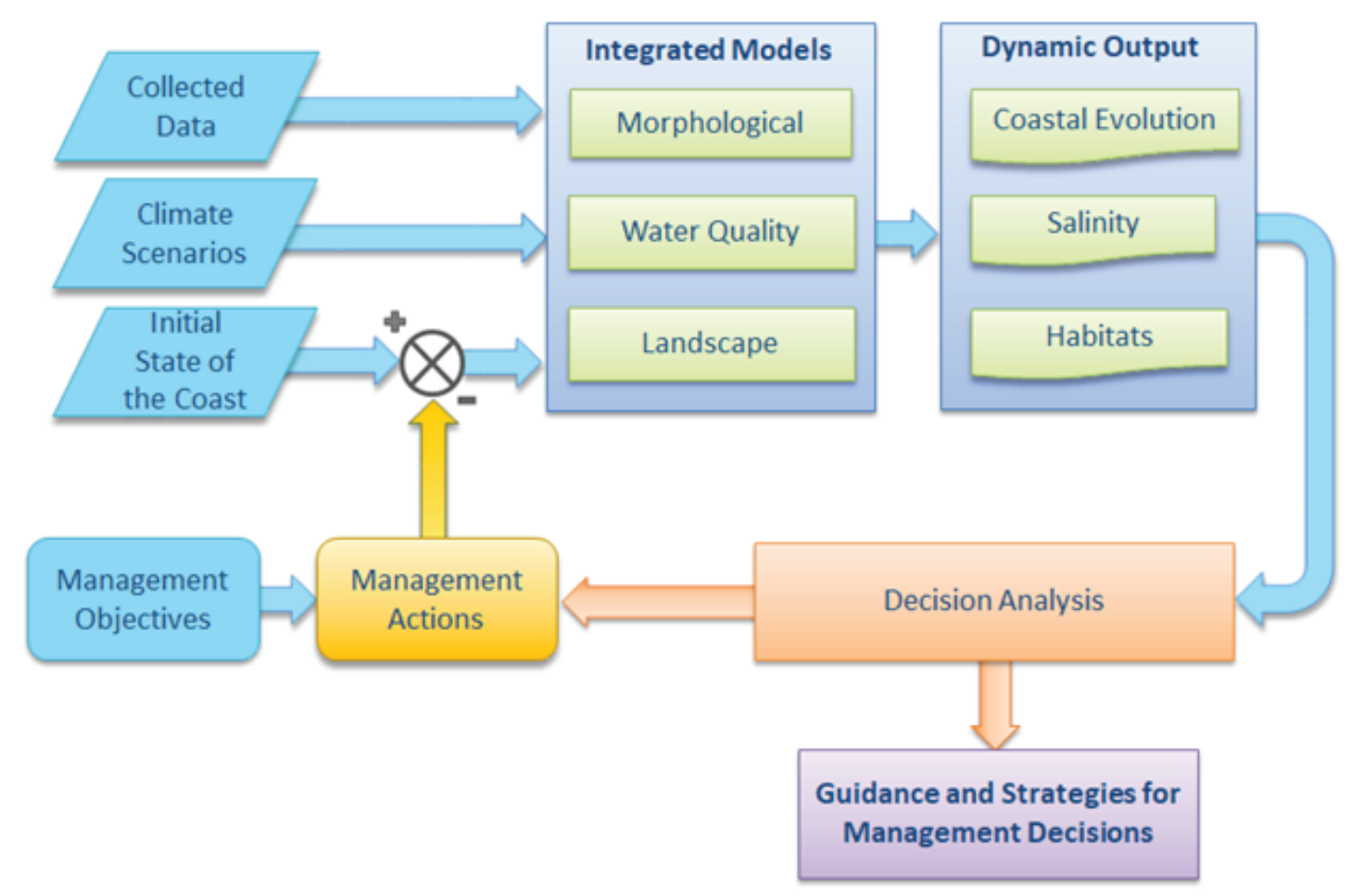

Figure 5.11. Overview of the Alabama Barrier Island Restoration Assessment study. 
This effort could be expanded by increasing our understanding of barrier island vegetation recovery and succession, including overwash response and marsh productivity and drowning, in response to sea-level rise and reoccurring storms. Insight from these analyses can be used to better calibrate models used in this study and lead to more thematic detail in habitat model outputs. In this study, we used expert elicitation to discern habitat importance to species of concern. Future work is needed to develop models that can be used to more explicitly understand how barrier island changes may impact important fauna (for example, shorebirds and sea turtles) that utilize these islands. Additional enhancements are needed and discussed more in the next section to increase the efficiency of data exchange between models and dissemination of outputs. Collectively, these enhancements could lead to more robust and efficient models that could be used to make operational decisions related to restoration, such as: (1) quickly running different scenarios; (2) addressing new morphologic change (for example, a newly formed breach); and (3) re-weighting stakeholder values in risk assessment.

\section{Operational Implementation and Technical Considerations}

The ALBIRA included a multidisciplinary, integrated science team that can support the planning, implementation, monitoring and adaptive management of barrier island restoration alternatives in Alabama. USGS also maintains the data storage, access and delivery of datasets and products to internal and external partners via a project site. For future efforts, there are opportunities to enhance data exchange workflows and model interoperability between modeling teams to increase the feasibility of operational implementation. For example, information exchange, particularly exchange between geomorphic and habitat models, could be enhanced through increased data interoperability and the use of open-source software and (or) developing scripts and tools to process information exchange between various model components. Additionally, the use of High-Performance Computing (HPC) would expedite numerical model simulations and allow for expansion to broader spatial scales and consideration of additional scenarios. As this analysis is expanded out to integrate more real-time analyses, tools could be developed to efficiently integrate and synthesize in situ observations, predictions of total water level and coastal change, and remote sensing data. Cloud services may be helpful for facilitating a more streamlined data exchange and decrease the latency of data product visualization when combined with custom open-source tools. This investment would not only help the science become operational but may also allow for more feedbacks between models (for example, development of post-storm vegetation recovery predictions for more accurate inputs for morphologic models).

\section{Longevity and Sustainability}

Coastal managers and decision-makers will continue to need scientific data regarding the potential impact of future coastal hazards (for example, extreme storms and sea-level rise) to make informed decisions for managing human and natural communities. Within the Gulf of Mexico, over $\$ 4.2$ billion in DWH funding has been allocated for the restoration of wetland, coastal and nearshore habitats, which our science can inform. This science requires integrated, multidisciplinary studies that incorporate various factors that impact coastal systems at local scales. The models developed for this study can be used to assess the present state and potential future conditions of barrier islands elsewhere. This could include assessments of the effect of future storms, sea-level rise, land use/land cover changes, and restoration alternatives such as hard structures or natural and nature-based features. Finally, the future-focused nature of this effort could be coupled with model outputs from assessments or predictions of the impacts of contemporary extreme storms for a full picture of the dynamic nature of coastal areas.

Primary Contact: Davina Passeri, dpasseri@usgs.gov; Nicholas Enwright, enwrightn@usgs.gov

\section{Mississippi Alluvial Plain Water Availability Study}

The Mississippi Alluvial Plain (MAP) Water Availability project, the pilot for the USGS Integrated Water Availability Assessments, is integrated across Water, Energy and Minerals, Hazards, and Core Science Mission Areas as well as our sister agency, Bureau of Reclamation, to coproduce water availability science with our stakeholders. The project uses a stakeholder driven approach to fully integrate water quality and quantity monitoring and modeling efforts across all hydrologic compartments to answer stakeholder questions in a timely matter and at a relevant scale (fig. 5.12). 


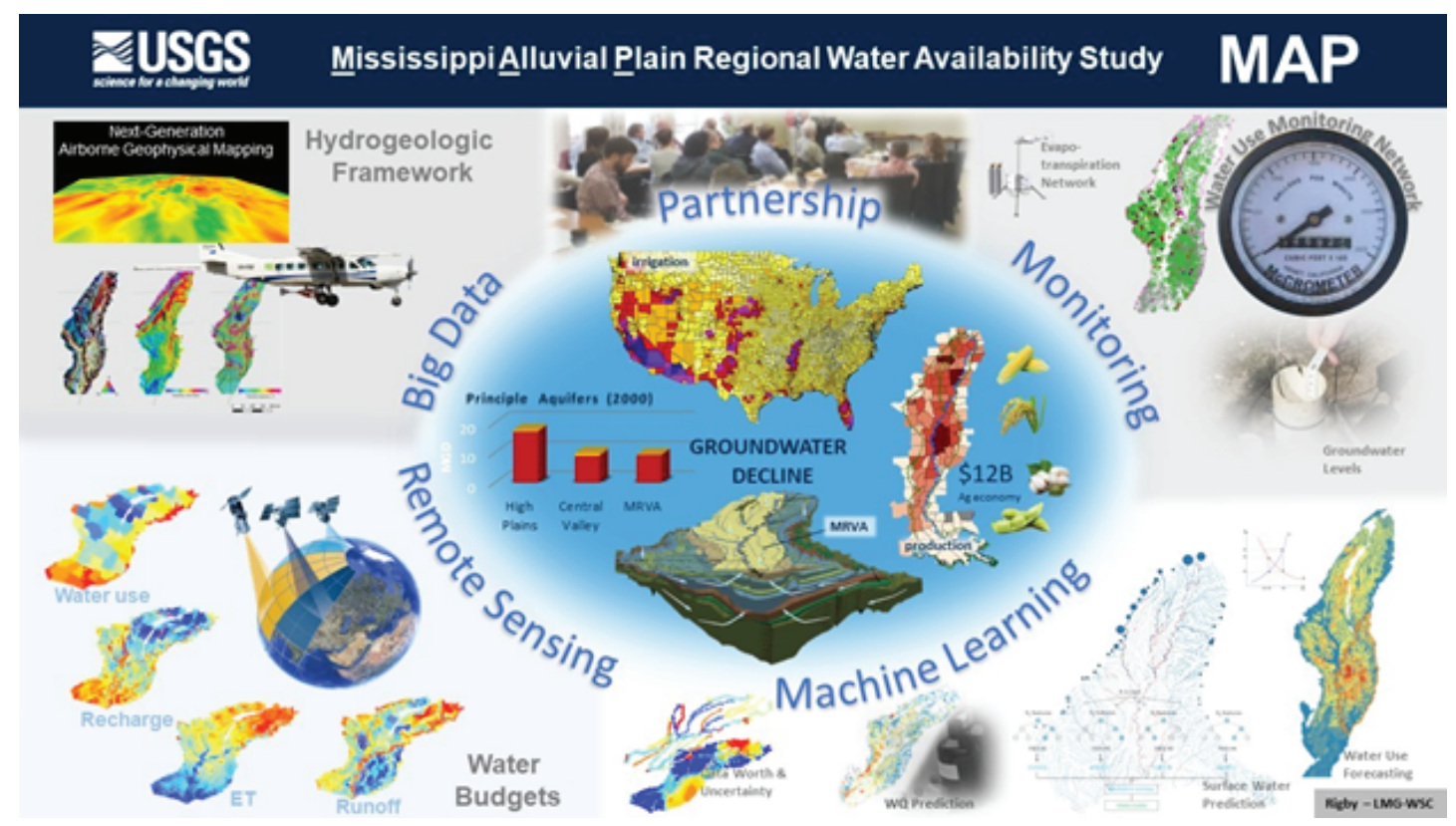

Figure 5.12. Conceptual diagram of MAP components and integration.

\section{Demand and Relevance}

Stakeholders are central to the Mississippi Alluvial Plain Water Availability project and have an active role in developing each year's workplan and scientific focus as well as assisting with community outreach efforts. Examples of local, state and federal stakeholders include Arkansas Natural Resources Department, Arkansas Department of Health, Arkansas Department of Environmental Quality, Louisiana Department of Transportation and Development, Louisiana Department of Environmental Quality, Mississippi Department of Environmental Quality, Delta Council, Delta F.A.R.M., Delta Wildlife, Yazoo Mississippi Delta Join Water Management District, Missouri Department of Natural Resources, U.S. Army Corp of Engineers (USACE), U.S. Department of Agriculture, The Nature Conservancy, University of Arkansas, University of Mississippi, Mississippi State University, Louisiana State University, Memphis State University. Through this intentional coproduction of information, the Mississippi Alluvial Plain Water Availability project has produced several examples of actionable science including; (1) collaboration with Hazards to provide data to evaluate the New Madrid seismic zone; (2) predictive modeling to inform a groundwater transfer injection project led by USDA and USACE; (3) development of a regional high frequency water use monitoring network with multiple partners; (4) working with the USACE to assess hydrologic infrastructure and ecological habitat and sediment transport in the Mississippi River; (5) optimization of surface and groundwater monitoring networks; and (6) coupling of hydrologic and economic models to inform stakeholders prioritizing water availability projects for the region.

Integrated science and information synthesis is a necessary component of the MAP project and includes the following:

a. Hydro-economics - integration of hydrologic models and socio-economic models and assessments

b. Integration of geophysical and component hydrologic models

c. Integration of remote sensing and physically based models

d. Co-production of decision support tools, integrating inputs and outputs of all modeling and monitoring efforts.

The MAP project directly addresses known stakeholder priorities through co-production of information (iterative product development). The MAP project then can address Program, Mission Area, bureau, departmental, and administration priorities by aligning and leveraging these priorities to meet Stakeholder needs. Examples include: 1.) the USGS Water Resources Mission Area (WMA) has a priority to predict water use at a daily time scale. This is also a priority to MAP stakeholders and can therefore be leveraged to meet both USGS and Stakeholder needs; and, 2.) DOI has a goal for the USGS to "Apply science to land, water, and species management"; the MAP project is directly addressing and advancing this goal with their Stakeholders, benefiting both DOI and local stakeholders. 
Modeling, analysis, and prediction in one of the most stressed hydrologic systems in the US demands a holistic approach that is scalable and efficient (near real-time science for near real-time decisions). For example, MODFLOW 6 allows for regional and local inset models to meet different needs (demands) related to difference scales and areas.

\section{Improved Decision-Support}

The MAP project has and continues to significantly improve stakeholder decision make via co-production of information and iterative science, allowing for improved investment in alternative water supply options and improved metrics for evaluating water availability in terms of current availability and changes in availability over time and space. Co-production of information with stakeholders, which involves describing problems and questions with stakeholders as the project is being proposed and developed requires frequent and regular engagement with stakeholders to go over preliminary models and output in order to ensure we are doing science with our partners and not to them. Additionally, the MAP project works with stakeholders to develop questions and scenarios that stakeholders want to understand to make decisions to secure future water resources (for example, how much will water availability decrease if we increase water use? Will water availability increase if we switch to alternative water resources? If we keep using water from this location, will we see saltwater encroachment?).

\section{Science Capabilities and Advancement Opportunities}

The MAP project is employing several 21st-century science capacities to achieve this goal; including the use of machine learning models for geology, groundwater, water-use, water quality, and streamflow; remotely sensed satellite and airborne geophysical data acquisition to inform and validate hydrologic models, integrated predictive modeling for scenario analyses and optimization including economic models, and decision support tools to help distill modeling and monitoring output to our stakeholders and the public.

Examples of existing foundational science capabilities include:

a. System-scale airborne geophysical surveys are enabling new capacity for mapping - 3D hydrogeologic structure. MAP has demonstrated this capability for the 1st time in the US, but highlights the need for developing an interdisciplinary community initiative to acquire national-scale subsurface data. Has potential to integrate with other large-scale airborne initiatives such as the Earth Mapping Resources Initiative (EarthMRI). Already interfacing with USGS Natural Hazards Mission Area and USACE for infrastructure mapping. b. Water-withdrawal monitoring in real-time providing verified irrigation activities for building relationships between withdrawals and drivers/triggers to define use. water withdrawal information available varied so greatly in the region due to state/local level management requirements. The metering/modeling effort is intended to consistently develop a method to identify irrigation activities and quantify the use uniformly for the region.

Advancement of science capabilities include:

a. Serving national/CONUS scale datasets that are "commonly" used in multiple models (soils, climate, etc.)

b. Machine learning models of water quality to quantify where water quantity is limited by quality

c. Water quality as a tool to identify recharge zones and variability in flow paths, especially in areas of high water use

Potential partnerships include: 1.) integrating state water-quality datasets (formerly done thru National Water Quality Portal [NWQP]), many states are not able to export data to the NWQP so there is a data lag between collection and consuming data into USGS models; and 2.) partnering with USACE and USDA on water availability issues, bringing all strengths to the table to provide the best information and products to stakeholders in a timely and economically efficient manner.

\section{Operational Implementation and Technical Considerations}

Science base is currently being used to help with delivery of products and serving datasets both internally (to USGS team members) and externally (to serve data to cooperators); stability of DOI links makes this achievable.

Internal:

- High performance computing (multiple modeling teams)

- Skilled work force for technical field sampling (meeting USGS Quality-Assurance/Quality-Control [QAQC] standards)

- Tools to efficiently track data collection and loading into NWIS and AQUARIUS

External:

- Access to private land and public supply systems for sampling and access for wells

- MAP has purposefully built an integrated science team that is intended to be greater than the sum of its parts through cross-team integration 
- Need to partner with software developers for better open-source tools for visualization and integration of multidisciplinary geological/geophysical/hydrologic data and models

- Think a bit more on timing of work elements in a Use Case like this... for example, airborne data acquisition should be planned a year or two ahead of other core activities like modeling so that work is not being done in parallel as much

\section{Longevity and Sustainability}

The MAP project is designed for long-term use, as it employs an iterative approach to provide the best modeling and monitoring information in near real-time while also continually working to optimize data collection and models. This requires multiple partners, funding sources, and engaged stakeholders. Since the MAP project addresses water availability in a holistic manner including stakeholders at the beginning of the effort, transferability to other areas or applications will be possible and is actively occurring; examples include: 1.) scripted workflows for pulling, processing, and serving datasets and models, which can be updated on the fly as new data are available (groundwater levels, water quality); 2.) Inset model tool for modeling water availability in higher resolution from regional-scale models; 3.) working towards an open-source workflow for airborne electromagnetic data, from acquisition/archiving to presentation and model integration; and 4.) the relationships built between irrigation withdrawal timing and quantity with drivers/triggers has transferability to regions of similar driver/ trigger/water use needs.

Information that will be used to evaluate the effectiveness of this effort and define metrics of performance and success include:

a. Intensity of stakeholder interaction

b. Use of products

c. Application of science on the ground (for example, GTIP)

d. Ability to bring together stakeholders from different agencies, states, etc.

e. Education and outreach opportunities

f. Stakeholder buy-in (Doing science with our partners)

g. Ability of partners to explain science to their stakeholders
Primary Contact: J.R. Rigby, jrigby@usgs.gov

\section{ALBIRA Acknowledgement Section}

The Alabama Barrier Island Restoration Feasibility Assessment was funded by the National Fish and Wildlife Foundation (project identifier 45719) to investigate viable, sustainable restoration options that protect and restore the natural resources of Dauphin Island, Alabama. This effort was completed in cooperation with the U.S. Army Corps of Engineers and in collaboration with the State of Alabama and the National Fish and Wildlife Foundation. This collaborative effort included efforts from many individuals from multiple agencies, including: (1) Elizabeth Godsey, Justin McDonald, and Brian Zettle from the USACE Mobile District; (2) Barry Bunch, William Butler, Victor Gonzalez, Thad Pratt, Michael Ramirez, Kim Sung-Chan from the USACE Engineer Development Research Center; (3) Julie Bernier, P. Soupy Dalyander, Nancy DeWitt, James Flocks, Jake Fredericks, Rachel Henderson, Robert Jenkins, Kyle Kelso, Joseph Long, Marci Marot, Rangley Mickey, Paul Nelson, Nathaniel Plant, Christopher Smith, Chelsea Stalk, David Thompson, Thomas Tuten, and Cathryn Wheaton from the USGS St. Petersburg Coastal and Marine Science Center; (4) Amy Gill and Richard Rebich from the USGS Lower Mississippi-Gulf Water Science Center; (5) Sinéad Borchert, Craig Conzelmann, Richard Day, Laura Feher, Christina Hunnicutt, Michael Osland, Michelle Meyers, Ann Hijuelos, Kathryn Spear, Spencer Stelly, and Hongqing Wang from the USGS Wetland and Aquatic Research Center; and (6) Kristie Coffman, Elise Irwin, Kelly Joyner, Quan Lai, and Clint Lloyd from the USGS Alabama Cooperative Fish and Wildlife Research Unit.

\section{Southwest Region}

\section{Enhancing Situational Awareness and Forecast Models for Post-Wildfire Runoff, Recharge, Water Quality, and Sedimentation}

Wildfires burning in the Western U.S. highlight key gaps in data and understanding that matter to local communities, as well as state and federal agencies. In the near term, those entities are looking for low-cost alerts for post-wildfire hazards and forecast models to help them prioritize erosion control to protect their water resources (fig. 5.13). Over longer time scales, communities need awareness of how landscapes recover from fire, in order to make decisions to protect water and ecosystem resources. 


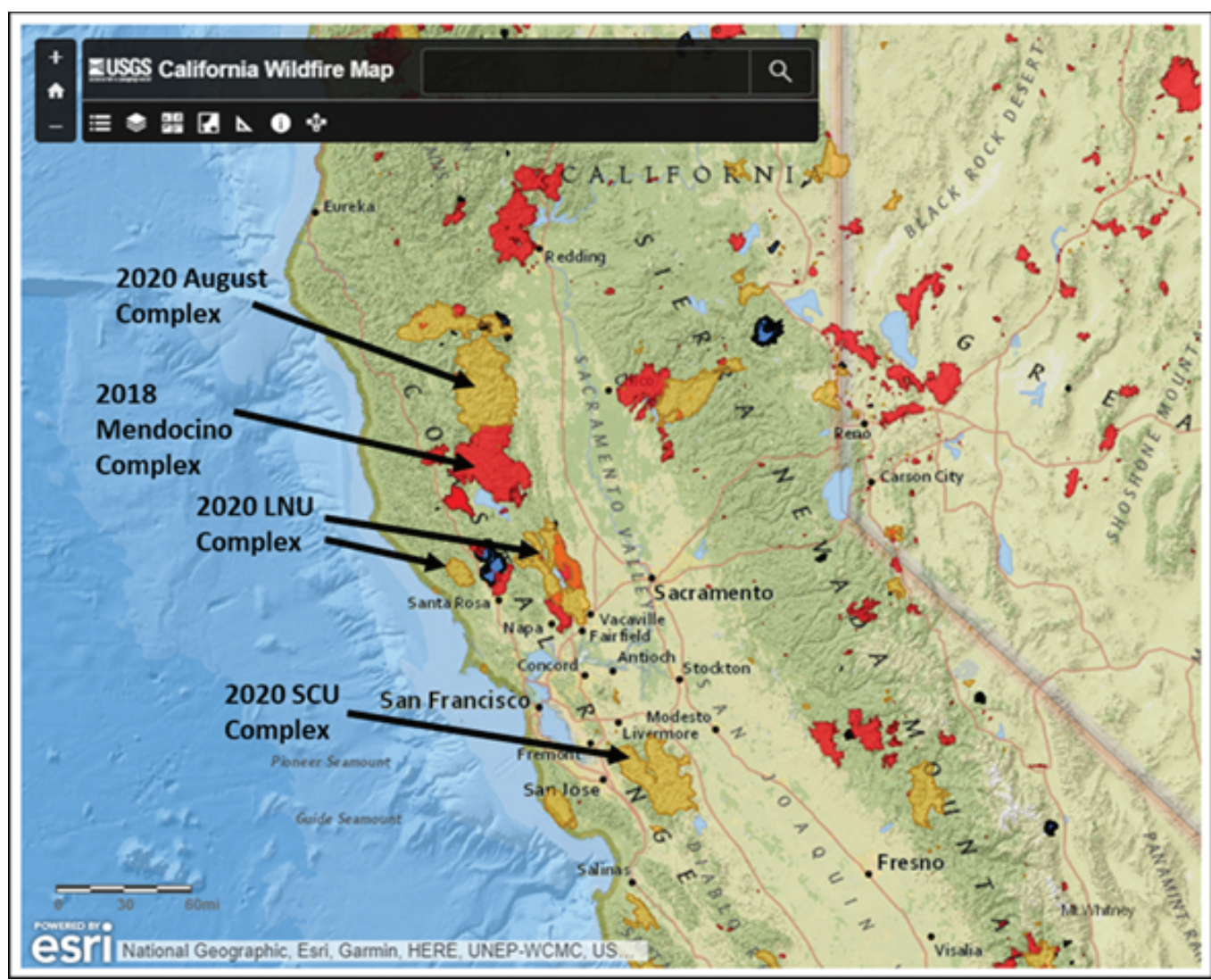

Map image is the intellectual property of Esri and is used herein under license. Copyright (C) 2020 Esri and its licensors. All rights reserved.

Figure 5.13. California Water Science Center interactive online California wildfire map showing northern California and Nevada wildfires in 2020 (yellow, includes LNU, SCU, and August Lightning Complex Fires as of September 10, 2020), 2019 (blue) and 2017-18 (red). On September 10, 2020, the August Complex became the largest recorded California wildfire, surpassing the 2018 Mendocino Complex Fire to its south. A large portion of the Coastal Range between the San Francisco Bay Area and Redding has burned in the last 4 years.

\section{Demand and Relevance}

Devastating wildfires and their after-effects are challenging the resources of local and state agencies in the west. These agencies must use their scare resources to protect lives and water resources from post-wildfire debris flows, runoff and sediment pollution from winter storms. The Bureau of Reclamation (BoR), U.S. Army Corps of Engineers (USACE), California Department of Water Resources (CA DWR), local utilities (PG\&E), Burned Area Emergency Response (BAER) teams and many county water management agencies have identified multiple needs for short- and long-term predictions of post-fire responses. These needs include:

1. How will I alert for flooding?

2. How much runoff can I expect, and for how long?
3. How much sediment can I expect, where will it come from, what is it composed of, and how will it impact channel ecosystem services, degrade water quality, or create other dangerous hazards downstream?

4. Will ecosystems and water quality recover quickly or do I need to make long-term investments, such as habitat restoration?

This Use Case will leverage the strengths of multiple USGS Science Centers and existing funding from Private and Public sources to build new partnerships to solve these post-wildfire challenges. Working together and with local, state, federal, and private partners, USGS scientists in the California Water Science Center (CAWSC), the Geology, Minerals, Energy, and Geophysics Science Center (GMEG), and the Western Ecological Research Center (WERC) are already in an excellent position to provide data and information needed to answer these questions. The USGS team has close working relationships with the partners in this Use Case and fully anticipates a co-development process. 


\section{Improved Decision-Support}

Currently local county agencies work with the BAER team to evaluate landscape response to burned areas and help in determining areas that are at risk of flooding, sediment pollution or debris flows. Local agencies then make decisions on how to protect infrastructure, inform local communities, and respond if a flood or debris flow takes place. Most local and state authorities don't have the expertise or tools available to monitor, forecast or predict what will happen in their watersheds. One of the few sources of real-time flood prediction is provided by the National Weather Service (NWS) at a very limited number of USGS streamgages (fig. 5.14). Unfortunately, the models used in these predictions do not incorporate changes in the landscape due to wildfire. Burn-severity, for example, is an important factor since highly burned areas can produce much more intense and damaging flooding. Post-wildfire flood predictions are also not readily available for many local agencies and may rely too heavily on outdated hydrological data or science. For example, in California, the BAER team frequently relies on post-wildfire flood predictions from tabulated values derived from a 1949

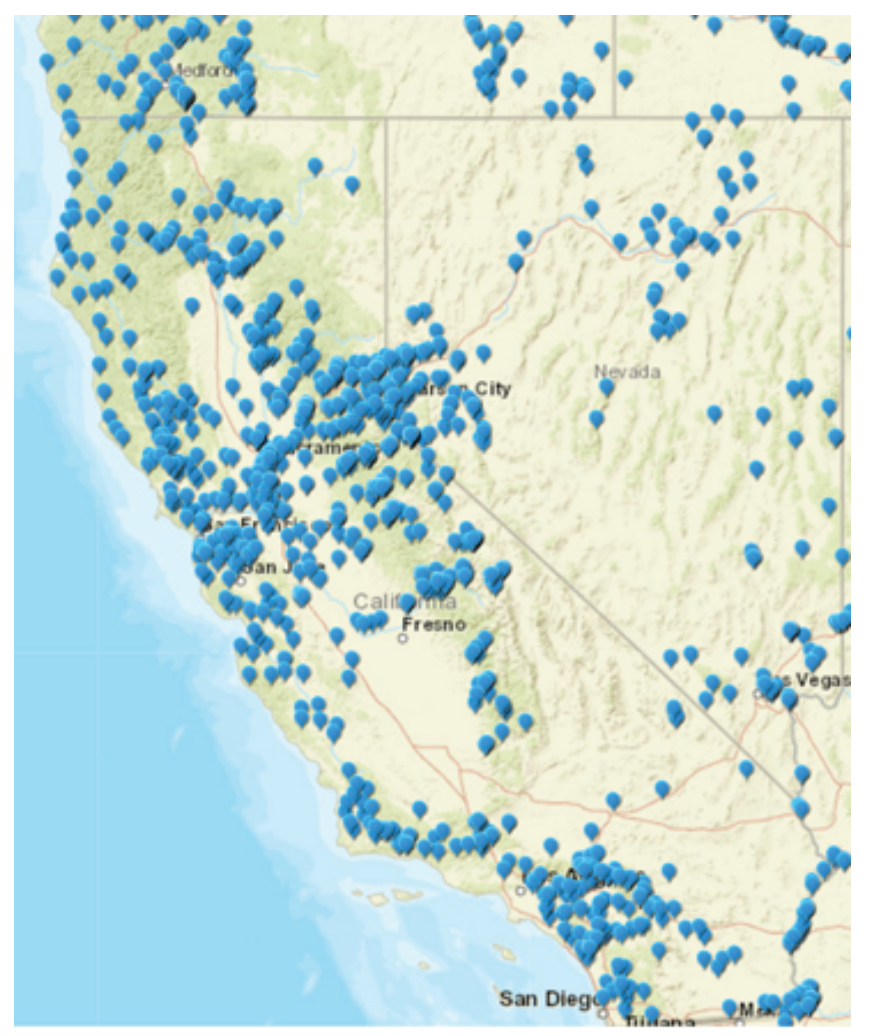

Figure 5.14. Inset map shows current streamgage locations in California, Nevada, and southern Oregon (blue dots).
USDA-Forest Service report. These data should be updated to incorporate the last 70 years of hydrologic data and more recent scientific understanding of landscape response as well as real-time streamgage data.

This type of information is vital to local and state agencies to predict which communities or infrastructure may be at-risk from floods. It also needs to be accurate and timely to avoid exposing the public to unnecessary risk while also avoiding "evacuation fatigue" from sending out too many unnecessary warnings. Real-time information is particularly relevant to emergency responders when a flood is occurring, but other local agencies may need to be more proactive about conveying flood risk, shoring up levees, protecting infrastructure, and finally warning the public to imminent threats. Most of the public investment to date has been in statistically based estimates of runoff responses but the emerging challenge of evolving western wildfire landscape is underscoring the need to shift investments to process-based forecast models.

\section{Science Capabilities and Advancement Opportunities}

The USGS currently has the scientific expertise to support this Use Case with experts in runoff modeling, hillslope and stream sediment transport, and streamgaging both here in California and nationally. The foundation of this new, integrated modeling effort will be real-time streamgaging and real-time flood inundation mapping in low-lying, susceptible communities. In addition, real-time rainfall runoff modeling would ingest quantitative rainfall forecast data (NWS already does this at some streamgages) as well as metrological and soil-moisture data. The model would also reflect changes to the landscape due to wildfire, particularly burn severity. The USGS has previously developed several distributed watershed runoff models in areas of California with recent and historical burns. Models for selected study areas would need to be updated to include a wildfire component. In addition, the BAER team uses other watershed models for emergency response such as those developed by the USDA-Agricultural Research Service (ARS) and USACE. After every fire, the parameters of the model would change and need to be updated. The streamflow models (for example: 1- to 7-day predictions) would not only forecast stage/ discharge at discrete points, but those points can be used in a flood-inundation forecast map to know where flooding will occur. As the USGS improves the scientific understanding of hillslope response in a post-wildfire landscape, we'll be able to gradually improve these prediction models. 
The USGS is also currently working on developing a low-cost streamgaging network system that can be deployed rapidly in a post-wildfire burn area (or anywhere) and can easily increase the number of gages in an area by an order of magnitude, in addition to incorporate other sensors (soil moisture, rainfall, water quality, etc.). These new environmental sensing data would feed into the real-time component of the post-wildfire EarthMAP as well as the forecasting component. The USGS has already formed partnerships with organizations that manage reservoirs and downstream habitat to improve understanding of long-term watershed responses to fire and has completed several studies specifically focused on their organization needs, including reservoir and downstream water quality, the effects of sedimentation on long-term water supply reliability, and below-dam fish habitat. Improvements to these process-based forecast models of sediment transport associated with post-fire runoff will be immediately useful for these longer-term questions as well.

The key areas for scientific advancement lie in pairing new fire-specific data with existing models to base short- and long-term forecasts on actual measurements, expanding the historical database of post-fire runoff, and improving the delivery of model forecasts.

The USGS already has formed many partnerships that will be leveraged for this post-wildfire EarthMAP Use Case, from technology development, to understanding the real-world management decisions that can be improved upon using these types of data. Both the USGS National Innovation Center (NIC) and CAWSC have partnered with Carnegie Mellon University and NASA to develop new sensor and telemetry tools. We've already partnered with Sonoma County to use their area as a test bed for these innovative streamgaging technologies. We're also regularly communicating with California Department of Water Resources to understand how we can improve situational awareness (related to flooding) in areas hit hard by recent wildfires.

\section{Operational Implementation and Technical Considerations}

This EarthMAP Use Case will leverage many capabilities that the USGS already has and is currently developing as well new technical capabilities which are within reach. Previous work, particularly at the TXWSC have improved visualizations of real-time streamgage data with real-time overlays of radar and other observational weather data. In addition, the CAWSC is currently working to expand the observation network for streamgaging and flood monitoring which incorporates recent advances in low-power computing and telemetry technologies. Perhaps more importantly, this team of USGS scientists already has strong relationships with the resource managers working on post-fire issues across California and a good understanding of their needs.

The NIC, DOD, and CAWSC have been working with Carnegie Mellon and NASA to deploy a low-cost, LP-WAN (Low Power Wide Area Network) system of non-contact stream-height sensors. These small, low-cost sensors report stream heights at 5-minute or shorter time increments using a spread-spectrum technology called LoRa, optimized for low- power consumption over low distances. Installation of added network uplink sites, around which many low-cost stream sensors can be sited, would provide real-time alerts for post-wildfire hydrologic risks, including flooding (Sonoma Water request). These sensors can be placed downstream of hillslope infiltration monitoring sites where measurements every 1- to 2-months will show changing infiltration thresholds for runoff and recharge, providing a watershed-scale check on hillslope data. Data showing runoff thresholds through time, validated by non-contact stream-height sensors, can be used to improve runoff and water availability forecast models such as the new hydrologic model developed by CAWSC and their partners, as demonstrated in the pilot project in the Russian River.

CAWSC and GMEG could leverage existing support from PG\&E, Sonoma Water, DWR, State Water Resources Control Board (SWRCB), and USGS NIC to extend post-wildfire infiltration monitoring networks and low-cost non-contact stream gaging sensors. Data showing infiltration rates and runoff response through time will be used to improve existing hydrologic models in the Russian River, improving post-wildfire flood and water availability awareness and forecasting. Support can also be leveraged from PG\&E and DWR/BoR/SFPUC (San Francisco Water Power Sewer)/ USACE to improve our ability to forecast sediment supply and subsequent transport that impacts reservoirs that supply drinking water for the San Francisco Bay Area communities. Data showing changes in hillslope sediment supply and stream transport will be used to improve forecasts of sediment threatening reservoirs, and to provide potential hillslope mitigation strategies. Data showing changes in stream channel metrics that matter to spawning and other ecosystem services will be compared to hillslope sediment supply measurements and models. The goal will be to improve forecasts of which channels have the highest susceptibility to post-wildfire impacts, in order to focus cost-effective mitigation efforts. 


\section{Longevity and Sustainability}

The need to improve situational awareness and forecast capability for post-wildfire flooding and water supply is an ongoing and cross-regional. Multiple aspects of expected scientific products will be transferable to other parts of the Western U.S. where similar monitoring networks, water availability, and sediment transport models are available. Specifically, the innovative low-cost, dense real-time observational network will be easily portable. The key to the scalability of this Use Case across California will be to clearly characterize how the extra investment in process-based forecasts can help resource managers reduce local risk, stabilize watershed resources, and improved regional water supply reliability by using the new science. Such an assessment must be led by our Public and Private sector partners, whose existing funding and support are keys to longevity and sustainability. The long-term viability of this Use Case will also depend on support from resource managers. Additional measures of success of this Use Case will be determined by whether the BAER teams and other resource management partners use the information products to communicate between different organizations and long-term assessments of hazard and risk reduction.

Primary Contacts: Anke Meuller-Solger, amueller-solger@usgs.gov; Jonathan Stock, jstock@usgs.gov

\section{A Drought Data Explorer for the Colorado River Basin: Integrated and Dynamic Web-Based Delivery of Actionable Information}

Intensification of drought across the Colorado River Basin is causing ecosystem stress and catastrophic transformations that result in increasing challenges for resource management. The Terrestrial Drylands Ecology (TDE) Branch of the Southwest Biological Science Center (SBSC) recognizes that dynamic delivery of integrated data, models and tools resulting from their collective dryland and drought-based research is needed to maximize the actionable utility of their science and to support the diverse stakeholders who rely upon this work. An essential step to increase the utility of this science is an interactive web-based Drought Data Explorer to help managers with their critical decisions to mitigate, adapt, and prepare for drought-induced changes to Colorado River Basin landscapes.

\section{Demand and Relevance}

Drought in the Colorado River Basin (hereafter Basin) has increased in frequency, intensity, and extent in the early 21 st century, and is expected to continue intensifying under climate change (fig. 5.15). Drought can severely degrade the ecosystems and natural resources that land managers are charged with sustaining for future generations. Despite the growing threat and effects of these interacting stressors, managers often have limited resources to effectively respond. While many adopt a "wait and see" or reactive management strategies, forward-thinking land and resource management is essential to prepare for impacts and allow for greater flexibility in decision making. Anticipatory management strategies are growing in importance, as abrupt and unexpected transformations of ecosystems have been increasingly observed and are likely to continue across the Basin. TDE scientists have produced models, datasets, and tools useful for decision making under increased drought. However, these products are generally project driven and have not been integrated in one platform that is accessible broadly to stakeholders.

The TDE researchers conduct cross-disciplinary science often in collaboration with Ecosystem and Water Resources Mission Area scientists in other Southwest and Rocky Mountain Region based centers and with academic partners nationally and internationally. They produce products that provide ecologically meaningful metrics of drought, understanding of how drought interacts with other stressors (wildfire, invasive plants and animals, insect outbreaks, etc.), evaluations of how drought and associated stressors impact natural resource conditions (for example, soil erosion and dust, ecosystem productivity and health, phenology, and water use), and assessments of how management actions can exacerbate, mitigate, prioritize, and (or) prepare for changes to natural resource conditions. Integration of existing and anticipated data and tools resulting from these scientific activities in a Drought Data Explorer will make drought-related information more readily accessible to our federal, state, tribal, and private stakeholders across the Basin. Drought and the need for salient and accessible information for management of the numerous ecological, safety, and public health and societal issues drought causes is a top priority from local to national levels of government and has been so identified within the USGS with a focus on drought in the Basin. 


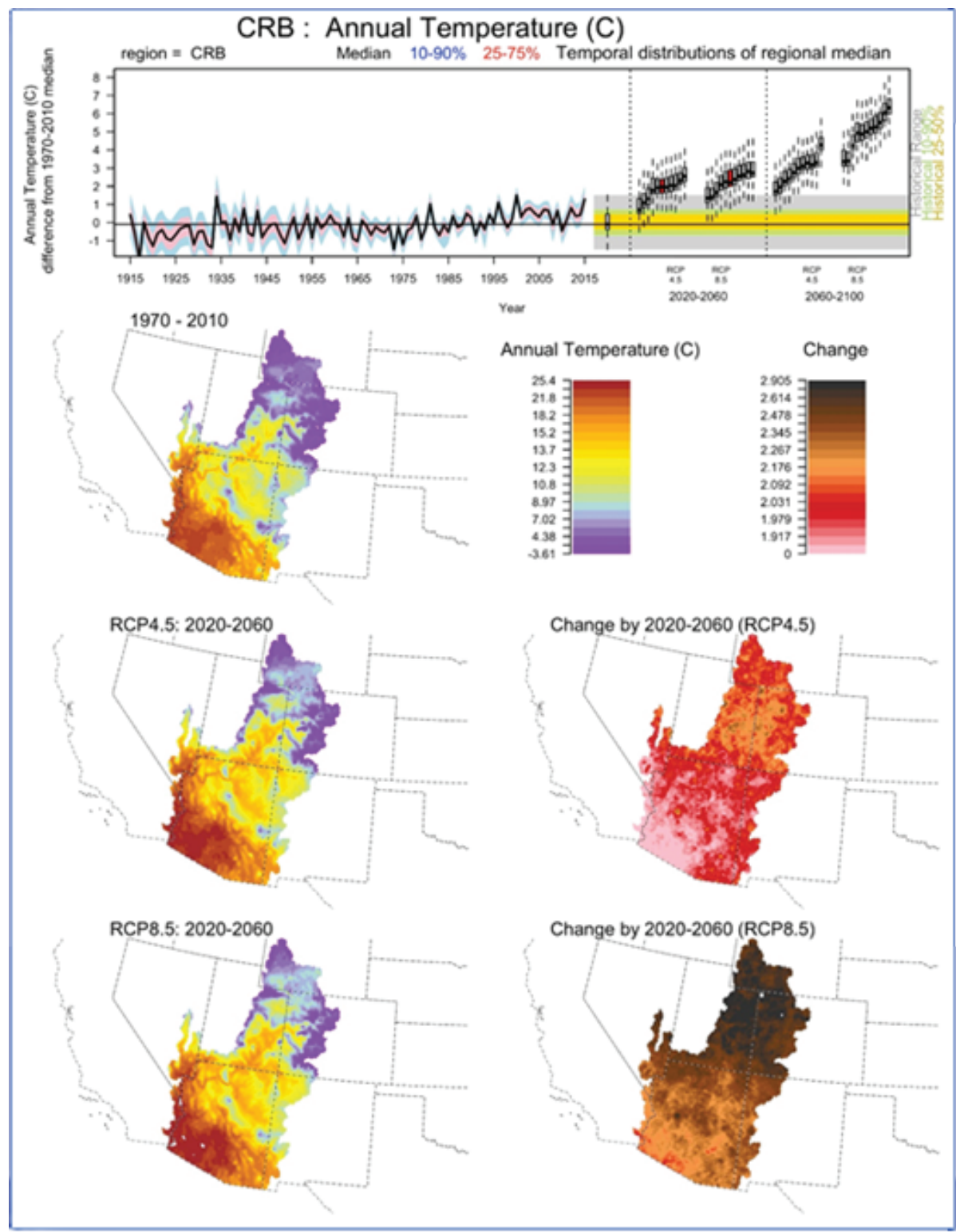

Figure 5.15. Increasing temperatures predicted for the Colorado River Basin (CRB) will result in long and enduring drought conditions. All components of the ecosystem will be responding to this stress and managers will require the type of data, models, and tools produced by U.S. Geological Survey scientists, particularly scientists working directly in the Basin. Graphics courtesy of John Bradford, SBSC TDE. 


\section{Improved Decision-Support}

A Drought Data Explorer delivering related data and tools will improve stakeholder decision making at different stages of drought intensity. The Drought Data Explorer platform can allow broader integration of drought-relevant science across the region, linking TDE produced products with related data from other centers and Mission Areas. Management decisions typically mirror ecosystem changes and when drought intensity is low, management typically follows a "business as usual" approach, in which management actions are conducted as they have been historically. As drought intensity increases, managers seek out and adopt mitigation approaches, which are aimed at minimizing the severity of these stressors, including planning activities to mitigate risk and conducting vulnerability assessments to target need for proactive management. For example, increasing drought intensity can trigger managers to adopt adaptation approaches in which they attempt to mediate the consequences of drought, such as altering livestock management, reducing tree or shrub density, treating invasive species, limiting soil disturbance, and other preventive measures. When ecosystem transformations are inevitable, land managers may prepare by reallocating resources to degraded areas and conduct ecosystem restoration, potentially including assisted migration of species.

An integrated Drought Data Explorer can be used to identify areas in the Basin that are susceptible to elevated temperatures, decreased rainfall, and low soil moisture. These projections can be then coupled with metrics of vegetation production, actual evapotranspiration, wind erosion vulnerability and dust emission risk, altered fuel loads, and wildfire risk; all developed or being developed by TDE researchers. The Drought Data Explorer will quantify the drought vulnerability of ecosystems under different land uses and management treatments, including projections of the impact of different management action scenarios. Once ecosystems have undergone transformations to degraded states, land managers typically switch their strategies to facilitate the replacement of species and communities that may perform well under the altered climate and wildfire regimes. A Drought Data Explorer could help prioritize areas of land with the potential to respond favorably to restoration and reclamation efforts and develop new methods and techniques to increase restoration success. While many of the preliminary data layers and tools that would contribute to a Drought Data Explorer have been or are being developed by TDE researchers, this information to support decisions are currently being presented to managers on a per-project basis.

The Drought Data Explorer will enhance the capacity of USGS to deliver cutting-edge, interdisciplinary science that serves the Basin's management community. TDE scientists have extensive experience engaging land and resource managers through one-on-one meetings, conferences, workshops, and field trips. These ongoing forms of stakeholder engagement have generated vast interest and great enthusiasm for developing novel and forward-thinking strategies to manage diverse ecosystems in the face of growing drought risk. The Drought Data Explorer will be developed from start to finish by maintaining these forms of engagement and the open communication TDE scientists have with managers in the Basin.

\section{Science Capabilities and Advancement Opportunities}

TDE scientists study how ecological drought manifests in the following specialties: climate and soil moisture; evapotranspiration in natural and degrade ecosystems; biogeochemical cycles and ecosystem productivity; soil erosion and dust emissions; vegetation composition and cover; plant and wildlife species of concern; changes to fuel load and wildfire activity; and invasive plants and insects. Also, extensive research and outreach are being conducted by TDE scientists in collaboration with land and resource managers to develop restoration and rehabilitation methods to address the consequences of drought and associated stressors. 
The products developed by TDE researchers are multi-faceted and will be additive when integrated within a Drought Data Explorer. For example, data and information ready to integrate, include applications of the SOILWAT2 ecosystem water balance model to assess temporal and spatial patterns of ecological drought, both in the past and in the future under a suite of climate change scenarios. An extension of this would be parametrization of SOILWAT2 with cutting edge predictive soil maps, recently developed by another TDE team, for the Basin above Lake Mead recently developed. Another example of data ready to incorporate into a Drought Data Explorer are those resulting from a collaboration with EROS and the Western Geographic Science Center. In that collaboration scientists have integrated a vast network of historical permanent vegetation plots across the Basin with Landsat and weather station archives to produce coupled data layers of drought effects on vegetation production. In an upcoming project, TDE researchers will integrate land surface, atmospheric, and hydrologic processes in the Weather Research and Forecasting model using remote sensing products to evaluate controls of these processes over the 57-year Landsat archive. TDE scientists are collaborating with the Geosciences and Environmental Change Science Center who maintain a dust monitoring network and a one-of-a-kind dust-on-snow sample archive which can be used for dust-source provenance and is readily available for model integration with TDE products. Another example of existing and supporting data are within PlotNet, which contains 11,000 upland vegetation and soil cover monitoring datasets from the western US, including the Basin and the results from RestoreNet, a networked experiment co-produced with land managers to improve restoration strategies across the Basin. TDE products being developed in collaboration with the University of Arizona, will provide high-resolution and dynamic change maps of greenness in both riparian and terrestrial Basin ecosystems and annual and growing-season evapotranspiration metrics. Through the work of TDE scientists and their collaborators, there is a large amount of soil moisture, land cover, land use, vegetation change predictions, vegetation water use, and soil data across the Basin that can be and should be integrated.

Partnerships will be strongly leveraged to make the Drought Data Explorer operational, including current partnerships with other USGS Science Centers (for example, EROS, FORT, Arizona WSC, WGSC, WERC, FRESC, GECSC), and with federal (BLM, BIA [Bureau of Indian Affairs], NPS, USFWS, DOE, DoD, FWS, USDA), state (Arizona Game and Fish Department [AZGFD], Arizona Department of Forestry and Fire Management, Utah Division of Natural Resources), tribal (for example, Navajo Nation Division of Natural Resources, Hopi Department of Natural Resources, Ute Mountain Ute tribe, and academic (for example, NAU [Northern Arizona University, UofA [the University of Arizona], ASU [Arizona State University], USU
[Utah State University], the University of Utah [UU], BYU [Brigham Young University], NMSU [New Mexico State University]) stakeholders throughout the Basin.

\section{Operational Implementation and Technical Considerations}

TDE scientists have substantial capability to support this Use Case with their ongoing field, lab, and modeling investigations, their strong relationships with agency and university scientists and management partners, and the research infrastructure they maintain in experiments, datasets, and equipment. While a great deal of this work is ongoing, with additional resources TDE could leverage synergies among, what are now, discrete research projects, and create a much-needed data and information delivery platform. The envisioned Drought Data Explorer will leverage data delivery frameworks that have been developed with individual projects. For example, an interactive, searchable Lower Colorado River Data Explorer is being developed within one project and is envisioned to be expanded to include additional river and upland ecosystems and transboundary, borderlands ecosystems. Other elements which have been developed with TDE science lead include the Smart Energy web platform and the DART tool.

In order for our research and outreach to evolve and become a fully functional and an operational service for scientists, land, water, cultural, and resource managers, and the general public, development of the Drought Data Explorer requires: a) adequate data storage, b) processing resources, c) web and tool developer time, and 3) long-term hosting. The Southwest Biological Science Center has made a commitment to advancing data science within the center and has supported the hiring of science support staff with the skills to develop advanced data delivery. We envision that the Drought Data Explorer can be developed with a modular design so it can accept new data and models as they are developed and is responsive to stakeholder needs for updated information.

\section{Longevity and Sustainability}

TDE scientists have been performing applied, drought-relevant research throughout the Basin for decades. Because of their strong partner relationships and history of success, the viability of this science is high, and the technologies developed are utilized currently and have high probability of generating continued need for use as long as water availability is an issue in the Basin. Adequate staffing is a primary need for this Use Case. The TDE permanent staff or researchers is small ( 8 researchers) and high productivity is achieved where technician and post-doc support can be maintained and adequate support staff (data and computer scientists) are dedicated to development of advanced data delivery and decision support tools. 
Drylands, including those in the Basin, currently cover 40 percent of the Earth's land surface and are rapidly increasing in size. Drought threatens to increase the area of the nation and globe that must be managed as drylands. The production and delivery of drought-related science productsthe data, tools, and models - will be a common goal of the managers of these lands and their resources. A Drought Data Explorer will make existing TDE science more available for utilization and can provide a technical model for integrated data delivery and decision support.

\section{Predicting Water Quality and Environmental Health in Rivers of the Colorado River Basin Affected by Wildfire}

Water quality in streams and rivers can be affected by runoff from burned areas. Pulses of fire residue, such as ash to surface waters, can create large sags in dissolved oxygen (DO) levels and $\mathrm{pH}$, as well as extreme highs in turbidity and specific conductance which can lead to significant die-offs in aquatic life. Understanding the different geochemical and biologic pathways that contribute to sags in DO concentrations is important to predicting how pulses of fire residue will impact aquatic ecosystems and water quality.

\section{Demand and Relevance}

Wildfires will continue to increase in severity and frequency due to drought and a warming climate. Wildfires are a significant threat to all of the Western U.S. Regions and understanding and predicting wildfire risks are priorities for all stakeholders of these areas. Dissolved oxygen (DO) sags due to wildfire ash and debris flows entering aquatic habitats can cause significant die-offs. Understanding how different chemical reactions from burned materials can produce DO sags is a needed first step in developing a predictive tool of stream water-quality change that will help federal, state, and local resource managers preserve protected or endangered species, maintain valuable technological resources, and provide early warning to water treatment facilities.

The proposed Use Case will develop an empirical relation between geology, fire intensity, geochemical modeling, and DO concentrations. Once this relation is quantified, EarthMAP is the appropriate tool to integrate water-quality monitoring, geochemistry, fire science, geology, soils, precipitation, stream flow, and ecology, to predict how future wildfires could impact DO concentrations in downstream aquatic habitats and water resources. If fully developed, this tool could also help document and predict the timing, severity, and duration of fire effects on water quality in downstream networks and predict levels of trace metal concentrations that can be toxic to ecosystems and degrade drinking water quality.

\section{Improved Decision-Support}

Predicting DO sags is important to the preservation of endemic and endangered aquatic species. Once empirical relations are developed, EarthMAP could provide stakeholders with actionable information on downstream aquatic ecosystems that are in danger of having acute DO sags due to the geology of the burn area coupled with the intensity of the fire. This information could be supplied before a debris flow initiates, providing ample time to translocate species and remove or secure technological resources. Moreover, a predictive tool for stream water-quality change could improve stakeholder decision making by targeting habitats and in-stream operational facilities that are or are not likely to be impacted by changing stream quality.

\section{Science Capabilities and Advancement Opportunities}

Water quality in streams and rivers can be affected by runoff from burned areas. Pulses of fire residue can create large sags in dissolved oxygen levels and $\mathrm{pH}$, as well as extreme highs in turbidity and specific conductance (SPC). These pulses have been known to cause die-off of aquatic flora and fauna. Monitoring streams and rivers for $\mathrm{DO}, \mathrm{pH}$, turbidity, and SPC is needed to develop an empirical model and predict the timing and magnitude of change caused by surface water runoff from burned areas to downstream ecosystems. SPC can be used to determine the removal of soluble constituents in initial discharge water from burned areas, and changes in $\mathrm{DO}, \mathrm{pH}$, and turbidity can identify subsequent or stronger discharge pulses. River networks in burned areas should be continuously monitored for water-quality parameters to help document the severity and duration of effects due to fire.

Understanding the geochemical and biologic pathways that produce sags in DO concentrations is important to predicting how pulses of fire residue will impact river networks. Two pathways have been suggested that likely account for DO sags. The first pathway is the chemical oxygen demand of the fire debris. If sulfides and reduced metals are present in the burn areas or in runoff debris materials, water can react with these materials and decrease DO concentrations in streams and rivers. The second pathway is the biological oxygen demand where organic materials in the burn area or in the surface water runoff remove DO from the water through aerobic decomposition. 
Multidisciplinary data from different burn areas are needed for modeling the reaction pathways impacting DO concentrations in aquatic ecosystems downgradient from wildfires. Data collected from soil, ash materials, water, and sediments from burned areas and downgradient surface waters is needed. Concentrations and oxidation states of metals, concentrations of dissolved organic carbon and particulate organic carbon as well as continuous monitoring of water parameters of $\mathrm{DO}, \mathrm{pH}$, turbidity, and SPC from each burn area would be used in geochemical modeling efforts. The model results will help to determine the reaction pathways that are driving changes in redox potential, $\mathrm{pH}$, and $\mathrm{DO}$ concentrations that would provide information on the cause of DO sag. These model results would then be compared to the mineralogy of surface geology from the burned areas, and fire intensity to determine a relation between geology, soils, fire intensity, and stream water quality, particularly DO concentrations. Once the relationship is developed this information will provide a predictive tool to evaluate how water quality and ecosystem health will be affected in future burn area scenarios.

Wildfire debris has impacted river systems across the Colorado River Basin (CRB), and many of these systems are already being monitored. Little research has used this data to determine the pathways that account for sags in DO concentrations during and after fire debris flows. A review of data collected by USGS and others would be done to leverage already existing data. Existing data from across USGS science centers and other federal, state, and NGO stakeholders should be collated into a database to ascertain if these historic projects have necessary information to inform the predictive tool development for the Use Case. Some examples of repurposing historic data collection activities to determine effects on DO concentrations in downgradient streams include USGS Nevada WSC long-term monitoring of Clear Creek near Carson City, Nevada, and the effects from the Clear Creek fire; Army Corps of Engineers, USGS, and academics at the University of New Mexico study on the Rio Grande and the effects from the 2011 Las Conchas fire in the Valle Caldera, New Mexico; and USGS California WSC and USGS Western Ecological Research Center [WERC] work on the 2009 Fire Station fire in the Angeles National Forest, California.

USGS currently has the capabilities to perform the work for the proposed data collection and to store data in National Water Information System (NWIS). Collecting, analyzing, quality assuring, and storing real-time data, and water, sediment, and soil sample analyses is relatively inexpensive and is being performed by many federal, state, local, and NGO stakeholders. Utilizing USGS information and leveraging other stakeholder data as furnished records is fairly routine. Geochemical modeling capabilities are available with the USGS developed PHREEQC model.

Once enough cases from historic and current data have been evaluated, a relation can be established between different variables to inform the predictive tool that would be developed using EarthMAP capacities. Geospatially linking geologic maps, soil maps, burn areas and wildfire intensity maps, and at-risk aquatic systems and water supply systems should be able to be defined quickly and efficiently with current capabilities. The communication to stakeholders about the findings from individual locations would be rapid. The development of the predictive tool would require leveraging capacities and integrating these capacities in EarthMAP over time.

\section{Operational Implementation and Technical Considerations}

A main goal of implementing the proposed Use Case is to collect data from burn and downgradient areas rapidly to provide initial datasets for determining stream baseline conditions, the timing and duration of water and sediment inflows from burn areas, the magnitude of change to stream water quality and the geochemical and biologic reactions that degrade water quality. The targeted stream systems would ideally have variable geologic materials of burn sites and fire intensity and could be selected from across the entire CRB. Because of the unprecedented quantities of fires across the CRB landscape, the Use Case could leverage systems that are already being monitored through NGWOS, WMA, or other stakeholder projects, with additional data collection activities added where needed. Evaluation of data and geochemical modeling are fairly routine and establishing the relation between the different variables would be done case by case.

Once relations between data are established, building a predictive tool is well suited for EarthMAP priorities. The predictive tool would leverage interdisciplinary data that already exists from multiple agencies (USGS and other DOI agencies, USDA, DOE, DOD, NGOs, and academic institutions) such as geologic surficial maps, river network information, precipitation maps, soils map, sensitive and endangered ecosystems maps, and fire distribution and intensity maps, along with real-time monitoring of DO, pH, SPC, and turbidity in tributary watersheds, water quality, sediment and soil sampling. Linking these datasets in EarthMAP, along with predictive relations, provides information on how aquatic ecosystem health and water quality would be impacted by DO sags cause by future wildfires. 
Bottlenecks in data collection activities for the Use Case would include understanding what data are being collected by different stakeholders at project sites and how USGS can access these data. Communication within the USGS and with other federal, state, local, and NGO agencies can be lacking, resulting in duplicate data collection activities and a wide range of data storage repositories and quality assurance procedures. To leverage resources, it would be ideal to have good communication with all agencies and flexibility in planned data collection activities for each project site. Additionally, having access to furnished records from other agencies may be difficult and internal USGS data approval slow. The Use Case would work with USGS and stakeholder data managers to create semi-automated processes for accepting furnished records into NWIS. Using a semi-automated process could help to move data through the procurement and USGS quality assurance procedures to ensure timely delivery of data.

\section{Longevity and Sustainability}

The long-term viability and sustained relevance of this Use Case is high. When complete, the Use Case would provide a predictive tool for land and resource managers to determine effects of wildfires on surface water DO concentrations, a primary driver of aquatic ecosystem health and a priority constituent for downstream water supply or treatment systems. Predicting the effects of wildfires on DO concentrations utilizes current data collection activities and capacities. Once the relations between geology, fire intensity, and DO is established, the predictive tool should be able to scale up to cover all burn area geographies. To determine effectiveness of the predictive tool, downgradient water quality in streams and water systems could be monitored to determine longer-term change, duration, and return to pre-fire conditions.

Primary Contact: Rebecca Frus, rfrus@usgs.gov

\section{Data and Modeling Platform to Support Forecasting California Water Project Effects}

Surface water diversions from the Sacramento-San Joaquin Delta serve 25 million California residents and irrigate millions of hectares of agricultural land, and their effects on wildlife and other natural resources have been a potent source of controversy for over 50 years. This proposal argues that a major USGS effort to draw together physical, chemical, and biological monitoring data streams in the watershed and to provide a set of peer-reviewed, public domain environmental models linking surface water flows, sediment dynamics, and physical geography to the distribution and quality of habitats and ecosystem services support for fish, wildlife, and birds would provide the best, most reliable scientific basis for California water management and planning decision making that has been available to date.

\section{Demand and Relevance}

Most of California is relatively dry, and most residents and nearly all of the state's highly productive agricultural sector depend on water delivery from two remote sources: the Central Valley and the Colorado River. Surface water operations in California's Central Valley are essential to serving those needs. Because demand exceeds supply except in wetter years, release and diversion curtailments needed to protect fish and wildlife and their habitat impose significant and often expensive limitations to water availability for human purposes, especially agriculture. Water operations limitations for environmental purposes occur at multiple time and space scales, ranging from long-term reservation of water behind dams to support off-channel wetlands and seasonal flow and temperature profiles in the Sacramento River system to narrower real-time adjustment of Delta flows and export rates to prevent undue entrainment of fishes and degradation of aquatic habitat within the Delta. This layered challenge to water operations, and the associated costs of foregone water delivery, create a strong and durable demand for data and analytical products that can help water and fish and wildlife managers assess the cascade of effects of operations decisions from the physical environment to the native species and ecosystems that depend on it. Good information can inform more efficient resource management decisions that conserve species and habitats while providing water for people and farms. Improved science can also elevate high-profile regional planning for a future burdened by both a growing population and regional hydrology that continues to shift due to global climate change.

We propose to develop a data platform that draws together relevant long-term monitoring datasets and monitoring streams representing the physical, chemical, biological, and ecological state of the Sacramento-San Joaquin and associated watersheds, and to build and maintain upon that platform an integrated environmental model suite to support forecasting the response of wetlands ecosystems, aquatic ecosystem services, and fish and wildlife distributions and abundances to water management and policy choices and in the presence of ongoing climate change and land use development. 
Because the fundamental goal of this EarthMAP proposal is to draw together a large array of relevant environmental data and develop from it an integrated model suite that can be applied to reliably forecast the ecological effects of water and related resource management decisions, we expect the effort will need to draw on the expertise of at least three USGS Mission Areas: Ecosystems, Water Resources, and Natural Hazards. Development of this Use Case will require collaboration with the Bureau of Reclamation and California Department of Water Resources, which manage the state and federal water projects in Central California, and the U.S. Fish and Wildlife Service, NOAA Fisheries, and the California Department of Fish and Wildlife, which implement of the Endangered Species Act, its California analogue, and have other resource stewardship mission responsibilities.

This Use Case directly addresses a central goal in the recent project descriptions and governing biological opinions pertaining to water operations in California: to support decision making that optimizes water operations while respecting the state's water rights system and land-use requirements, and not threatening the continued existence or critical habitat of state and federally protected plant and animal species. It is a goal that the involved agencies and others have, for at least the last two decades, spent more than $\$ 50$ million in science support dollars annually pursuing, most of it to field a large suite of long-term monitoring projects spanning the gamut from hydrodynamics and water quality to fish and bird abundances and distribution. These field programs have provided a magnificently detailed data resource for the Bay-Delta that is not available in most other systems. Some of the physical and chemical monitoring is conducted by the USGS; as well, we are relied upon by all the agencies named in this proposal for advice and assistance with the development of models, visualizations, and other analytical products needed to support various operations and public policy decisions. These partners are likely to be extremely supportive of a thoughtfully developed USGS plan to build a data platform and system of integrated environmental models to forecast water operations effects on ecological services and the habitats and native species living within the system.

\section{Improved Decision-Support}

We expect that this EarthMAP application would benefit stakeholders and their decision making in several ways.
1. Forecasts. At present, Central Valley water operations decisions to achieve environmental objectives are generally not based on quantitative forecasts, and some are based only on rules of thumb; this approach would offer an alternative methodology expressly designed to quantitatively forecast the integrated effects of complex interconnected processes that arise from surface water operations.

\section{Co-production and decision support benefits. We} envision that agency staff would be partners in the development of applications of the water operations integrated environmental model; the collaboration would ensure that products are on-point and useable by stakeholders while also providing in-house expertise regarding their use within their agencies.

3. Reliability. Use of the common data platform and analytical framework envisioned here would increase confidence that analytical products perform as intended, with predictable characteristics and error distributions.

4. Timeliness. The envisioned platform would make it easier and quicker to develop analytical products than would otherwise be the case, allowing for faster, substantive decision support in short turn-around situations. Such situations are not rare.

5. Transparency. We expect that this Earthmap application and its derivative analytical products would be publicly accessible; the common platform elements would enhance stakeholder and (informed) public understanding of the uses and limitations of the platform and its products.

6. Defensibility. We expect that the application and its derivative products would be broadly subjected to formal expert review processes, making it easier for stakeholders to adopt the products in important and potentially controversial official decisions.

7. Portability (maybe). We see a significant chance that the approaches adopted in implementing this Earthmap application could be applied in or shared among other estuaries with a strong USGS presence, such as the Florida Everglades, the Chesapeake Bay and Watershed, the Delaware River system, and Puget Sound/Salish Sea. The Everglades system is particularly similar to the Bay-Delta in being driven by tension between habitat conservation and water supply goals. 


\section{Science Capabilities and Advancement Opportunities}

The core foundations required for this EarthMAP application are already available. Data management is "old hat" for USGS, and both the skilled personnel and some of the relevant data are already housed at the California Water Science Center. Relevant Sacramento-San Joaquin watershed monitoring programs and historical datasets are numerous; most are concentrated at a few locations, including the California Data Exchange (CDEC), the Interagency Ecological Program, and within our California Water Science Center. The water operations-focused integrated environmental model suite we envision in this proposal has already been prototyped as an objective of the USGS CASCaDE (Computational Assessments of Scenarios of Change for the Delta Ecosystem) projects. The application we envision here is perhaps somewhat less daunting than CASCaDE in that the focus is exclusively on water operations effects in the Delta and valley tributaries. The degree of difficulty would depend on details of the forecasting capabilities and other factors that we would have to sort out in collaboration with stakeholder agencies during implementation. It is possible, even likely, that advancement of this project would lead to the identification of new monitoring needs or changes to existing monitoring programs in order to appropriately serve the model suite.

This project offers the promise of integrating and curating the relevant data sources across disciplines and organizations expressly to support the envisioned integrated environmental model suite, and to erect a purpose-built model suite to support the range of quantitative water operations-related questions that are the core interest of this proposal. In all, the Use Case we envision would represent an exciting extension of the integrative, multidisciplinary work USGS has already done to address management challenges in this region to date, and offers a chance to provide clearly better tools to stakeholders charged with making high-profile, high-value decisions pertaining to California water, ecosystems, and species.

\section{Operational Implementation and Technical Considerations}

We anticipate that implementation of this proposal would require a supra-regional team effort engaging scientists and data managers at the California Water Science Center (CAWSC), the Water Resources Mission Area branch staff in Menlo Park and at Moffett Field, the Pacific Coastal and Marine Science Center, the Western Geographic Science Center, the Western Ecological Research Center, the Western Fisheries Research Center, and possibly others. Because success depends on accurately linking the envisioned capabilities to specific partner/stakeholder requirements, we expect that a strong collaborative steering engagement with Reclamation, the California Department of Water Resources, the Delta Stewardship Council, the State Water Resources Control Board, and the three federal and state fish and wildlife agencies would need to be organized at the outset to ensure that specific first-generation products and realistic timelines could be identified very early on. We do not anticipate any new technology needs at this time, though there may be a need for AI/ML support depending on the implementation objectives. Data platform requirements can be developed in collaboration with stakeholders.

\section{Longevity and Sustainability}

This EarthMAP Use Case is inherently a long-term proposition, and gains value as the commitment is extended. The tension between California water management imperatives has existed for decades and will unquestionably continue for the indefinite future. We believe that careful, effective implementation of the proposed data platform and integrated environmental model suite would create an analytical resource that stakeholders would adopt and internalize in their planning and decision processes, improving water management for the benefit of the public and creating lasting demand for both the products and USGS expertise and technical assistance. It is plausible that progress on the envisioned Use Case in the Bay-Delta could lead to expansion of the Use Case to cover multiple estuaries, though it has high value even as a stand-alone effort. We expect that the most appropriate performance metric for the proposed Use Case is the degree of adoption of its products by Bay-Delta stakeholders, and their reliance upon them in mission-critical resource management decisions.

Primary Contact: Mike Chotkowski, mchotkowski@usgs.gov

\section{Improving Daily Modeled Estimates of High Elevation Snowpack to Boost Runoff Forecast Accuracy in Colorado Basin Watersheds}

Effective reservoir management in basins where runoff is dominated by spring and summer snowmelt is often hindered by inaccurate estimates of patchy high elevation snow water equivalent (SWE), which is typically not represented by in situ observation networks and is poorly resolved by coarse resolution modeling and remote sensing. A combination of higher spatial resolution physics-based modeling and remote sensing can be used to more accurately estimate high elevation SWE, improve snowmelt runoff forecasts for the basin, and enable better reservoir management decisions. 


\section{Demand and Relevance}

Many reservoirs across the Western United States are fed primarily by runoff generated by high elevation alpine snow cover in the spring and summer months. Accurate predictions of snowmelt runoff that will occur during the late spring and summer months allow managers to optimize reservoir operations, water deliveries, hydropower production, and other water management objectives, such as balancing supply and demand.

Improved accuracy for estimates of high elevation stored snow water equivalent and runoff will also facilitate management of aquatic species that require minimum flow volumes or stream temperatures. With higher confidence in the volume of runoff expected to be generated from remaining high elevation snowpack in the spring, reservoir managers would have more leeway to release water earlier in the year in order to meet aquatic species management objectives, such as approximation of a natural flow regime.

Finally, flood forecasters would also benefit significantly from improved estimates of stored snow water equivalent. Over the short term (daily to weekly), flood forecasters would be able to more accurately predict near term hazards by combining near term weather forecasts with gridded snow water equivalent to predict runoff and identify potentially hazardous situations. Over the medium term (monthly), flood forecasters could use gridded estimates of snow water equivalent to drive simulation models in the early spring that would help identify specific weather events (for example, a large rain-on-snow event, a rapid-onset sustained warming event) likely to result in hazardous flooding well ahead of the potential onset of these events. This could allow for earlier warning of potential flood events. Notably, once validated, the Use Case described here could provide these benefits not only for basins feeding reservoirs, but for all basins with high elevation snow cover, including basins lacking the in-situ instrumentation that would otherwise aid in flood prediction. This could enable more specific flood predictions aimed at specific smaller basins.

Making predictions of spring and summer snowmelt runoff is notoriously difficult in basins that include a substantial amount of terrain above the alpine treeline. In these basins, estimates of stored snow water equivalent (SWE) that drive runoff forecasts are often deficient for several reasons. First, automated existing in situ observations of SWE (such as those provided by the NRCS SNOTEL network) are typically only available from lower elevation sites below the alpine treeline and may not reflect snowpack conditions at the higher elevations. Second, modeling and remote sensing used to estimate SWE is generally conducted at relatively coarse (500-1000 m) spatial resolution that is not sufficient to resolve the mosaic of patchy alpine snow cover that generates the majority of the late spring and summer runoff. The combination of these two factors often leads to inaccurate estimates of high elevation SWE. Novel approaches such as those developed for NASA's Airborne Snow Observatory
(ASO) have been demonstrated to be highly effective, but require regular airborne surveys that may be cost prohibitive in many areas. Federal agencies (such as the Bureau of Reclamation) and local water districts responsible for reservoir management, as well as agencies responsible for issuing snowmelt runoff forecasts (such as the Natural Resources Conservation Service), stand to benefit significantly from an approach that can improve the accuracy of snowmelt runoff forecasts without requiring expensive regular airborne surveys. The primary benefit of the approach proposed in this EarthMap Use Case would be increased accuracy in seasonal runoff forecasts for the upcoming spring and summer months of the same year. In addition, the proposed Use Case could also inform longer term decision making by reservoir managers when used to simulate the volume and timing of snowmelt runoff that would be expected over the longer term (decades) under different climate conditions as well as in the case of substantial land cover changes.

\section{Improved Decision Making}

The approach proposed here has the potential to improve both the spatial resolution and accuracy of stored SWE estimates for basins with substantial above-treeline terrain. Developing and implementing a standardized modeling and remote sensing approach and resulting data product would be of great benefit to reservoir managers in districts with more limited financial and technical resources. This data product would consist of a regularly updated near-real time gridded estimate of SWE produced by a validated modeling and remote sensing approach that would otherwise be beyond the capability of reservoir managers who did not have access to a staff with the capability to develop and apply a complex, physically-based model that incorporates regular updates from spaceborne remote sensing. This approach would deliver a product that reservoir managers from the Bureau of Reclamation and local water districts have indicated would allow them to make better, more informed reservoir management decisions in basins where spring and summer runoff is generated from patchy, high elevation snow cover.

\section{Science Capabilities and Advancement Opportunities}

The USGS currently (2020) has the capacity to implement the proposed remote sensing and physically based modeling approach. The needed datasets, key models, and remote sensing techniques that will form the backbone of the approach are already available from a combination of sources within and outside of the USGS. Successful implementation of the approach will require developing a standardized approach for acquiring the required input datasets and remote sensing imagery as well as incorporating them into the physically-based snow evolution model. 
The scientific and technological capacity to implement this Use Case exists primarily within the USGS. Dr. Graham Sexstone (Colorado Water Science Center) has extensive experience with implementation, calibration, and validation of physically-based snow models, such as SnowModel, developed by Dr. Glen Liston (Colorado State University). Dr. David Selkowitz (Nevada Water Science Center) lead the development of the recently released USGS Landsat Level 3 Fractional Snow Covered Area product and is currently working to create a combined Landsat-Sentinel fractional snow covered area product that would provide a much higher temporal resolution than the existing Landsat product. USGS employees and contractors based at the EROS Data Center have extensive experience with the production and distribution of Landsat-derived datasets and are currently developing a Sentinel 2 surface reflectance product that would enable the production of the combined Landsat-Sentinel snow covered area product. Additional datasets required for implementation of the physically based snow model include the National Elevation Dataset and the National Land Cover Database (land cover and forest canopy layers), produced by the USGS, as well as the North American Land Data Assimilation System (NLDAS-2) available from NASA. Daily in situ SWE measurements from the Natural Resources Conservation Service SNOTEL network would also be used for calibration and validation of this approach (fig 5.16).

Future improvements to required remote sensing and modeling datasets have the potential to increase the accuracy of modeled snow water equivalent. In particular, increases in the spatial resolution and accuracy of the National Elevation Dataset, such as those associated with the ongoing and expanding acquisition of LiDAR data across the nation, would improve the accuracy of simulated wind redistribution of alpine snow cover. Higher frequency updates of the National Land Cover Database (land cover and forest canopy layers) could also lead to additional increases in the accuracy of modeled SWE. Finally, daily streamflow observations available from USGS will serve as a crucial dataset for validation of modeled snowmelt runoff. Streamflow observations may also be augmented by the inclusion of reservoir inflow data from agencies such as the Bureau of Reclamation as well as local water districts.

\section{Operational Implementation and Technical Considerations}

The technical capacity to execute the proposed Use Case is currently available within the USGS. Development of the combined modeling and remote sensing approach would be conducted by Sexstone and Selkowitz, who will soon begin work funded by the Bureau of Reclamation for related work in two pilot basins in Colorado. Scaling the approach to a larger number of basins in the Western United States would require high performance computing (HPC) resources and staff, and near real time implementation of the approach for numerous basins would require the expertise of staff at EROS who have extensive experience producing and distributing regularly updated gridded datasets.

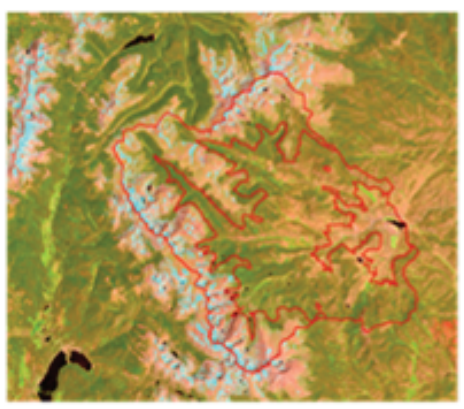

Landsat Surface Reflectance

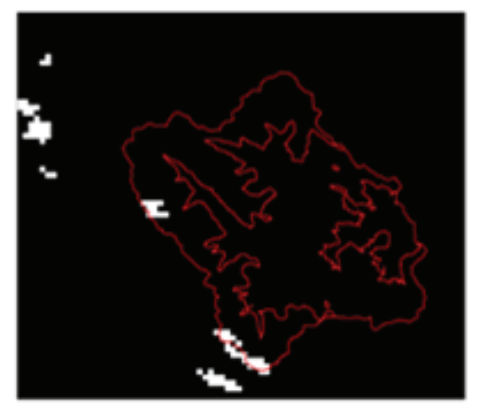

MODIS SCA $(500 \mathrm{~m})$

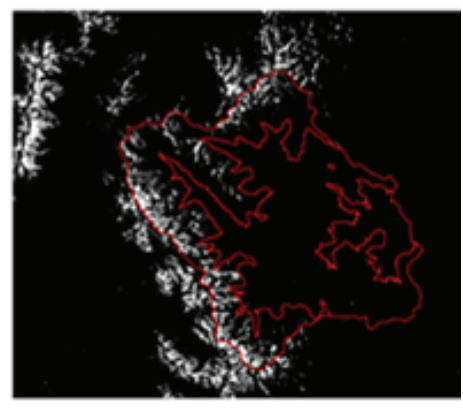

Landsat SCA $(30 \mathrm{~m})$

Figure 5.16. Coarse resolution remote sensing is not well suited for capturing patchy alpine snowpack that represents a large fraction of spring and summer runoff in some basins. In the example above covering a portion of Rocky Mountain National Park in Colorado, the 30-m Landsat snow covered area product identifies substantial high elevation snow cover not captured by the 500-m MODIS snow cover product. Assimilation of higher resolution snow covered area data derived from Landsat or Sentinel 2 data into a high-resolution modeling approach can substantially improve the accuracy of runoff forecasts in basins where forecasting has been particularly challenging in the past. 


\section{Longevity and Sustainability}

We anticipate this Use Case will be highly relevant over the next several decades. High mountain snowpacks currently serve as a large natural reservoir that fills up over the winter and spring and then delivers water over the period of highest demand in the summer. Projections indicate the timing of snowmelt will be earlier in the spring across much of the Western U.S., resulting in a larger mismatch between peak flows and peak demand later in the summer. Optimization of reservoir operations would thus become even more crucial, with little room for error. At the same time, climate conditions outside the range of conditions covered by the historical record of in situ snow observations may lead to decreased reliability of statistically-based snowmelt runoff prediction approaches, such as those currently used by the Natural Resources Conservation Service. In extraordinarily warm years, significant snow accumulation could even be confined entirely to the highest elevations which lack a historical record of in situ SWE observations. In this situation, statistically-based forecasting approaches would no longer be viable, while the physically-based modeling and remote sensing approach described here would continue to be effective.

The ability to sustain the modeling approach proposed here would be dependent on continuous acquisition of the USGS Landsat and European Space Agency Sentinel 2 remote sensing data. With a modest effort, the approach could be adapted to work with other optical remote sensing instruments that offered similar spectral bands, spatial resolution, and imaging frequency. Additionally, continued near real time production of the NASA NLDAS-2 model would be necessary, although the Use Case approach could be adapted to use an alternative model that produced similar outputs.

Once validated, the approach described here could also be adapted for implementation in high elevation watersheds outside of the United States. Estimates of stored snow water equivalent derived from physically based modeling and remote sensing for basins lacking in situ instrumentation or a record of historical snow cover observations would enable runoff forecasting that would not have been possible otherwise.

The primary metric for evaluation of this Use Case would be validation of modeled snowmelt runoff from stream gauges and reservoir inflow monitoring. The accuracy of the modeled snow water equivalent could also be directly evaluated via comparison with airborne snow survey results for basins where this type of data became available. The ultimate measure of success for this Use Case, however, would be increased snowmelt runoff prediction accuracy in basins where existing forecasts had previously been relatively poor.

Finally, it is worth noting that the science and technology developed for implementation of this Use Case would have broad applicability for a variety of problems where a combination of high spatial resolution remote sensing and physically-based modeling could be used to more accurately monitor a specific hydrological or ecological process. The combination of remote sensing data from Landsat and Sentinel 2 remote sensing data would be particularly applicable for monitoring irrigation and evapotranspiration.

Primary Contact: David Selkowitz, dselkowitz@usgs.gov

\section{References Cited}

Alexander, R.B., and Gorman Sanisaca, L., 2019, RSPARROW-An R system for SPARROW modeling [Software release]: U.S. Geological Survey data release, https://doi.org/10.5066/P9UAZ6FO.

Ator, S.W., 2019, Spatially referenced models of streamflow and nitrogen, phosphorus, and suspended-sediment loads in streams of the Northeastern United States: U.S. Geological Survey Scientific Investigations Report 2019-5118, 57 p., https://doi.org/10.3133/sir20195118.

Berman, E.E., Graves, T.A., Mikle, N.L., Merkle, J.A., Johnston, A.N., and Chong, G.W., 2020, Comparative quality and trend of remotely sensed phenology and productivity metrics across the Western United States: Remote Sensing, v. 12, no. 16, 27 p., https://doi.org/10.3390/rs12162538.

Cartwright J., and Johnson H.M., 2018, Springs as hydrologic refugia in a changing climate? A remotesensing approach: Ecosphere, v. 9, no. 3, 22 p., https://doi.org/10.1002/ecs2.2155.

Crausbay, S.D., Betancourt, J.L., Bradford, J., Cartwright, J.M., Dennison, W.C., Dunham, J.B., Enquist, C.A.F., Frazier, A.G., Hall, K.R., Littell, J.S., Luce, C.H., Palmer, R., Ramirez, A.R., Rangwala, I., Thompson, L., Walsh, B.M., and Carter, S., 2020, Unfamiliar territory-Emerging themes for ecological drought research and management: One Earth, v. 3, no. 3, p. 337-353, https://doi.org/10.1016/j.oneear.2020.08.019.

Delie, J. and Biedenweg, K., 2019, Understanding stakeholder values and perspectives for management of Malheur Lake: U.S. Fish and Wildlife, Malheur National Wildlife Refuge, 26 p., https://kellybiedenweg.weebly.com/uploads/9/4/0/6/ 94065145/understanding_stakeholder_perspectives_for_ malheur_wildlife_refuge_2019.pdf.

Dunham, J.B., Angermeier, P.L., Crausbay, S.D., Cravens, A.E., Gosnell, H., McEvoy, J., Moritz, M.A., Raheem, J., and Sanford, T., 2018, Rivers are social-ecological systems-Time to integrate human dimensions into riverscape ecology and management: WIREs Water, v. 5, no. 4, 10 p., https://doi.org/10.1002/wat2.1291. 
Eagles-Smith, C.A., Willacker, J.J., Nelson, S.J., Flanagan Pritz, C.M., Krabbenhoft, D.P., Chen, C.Y., Ackerman, J.T., Campbell Grant, E.H., and Pilliod, D.S., 2020, A national-scale assessment of mercury bioaccumulation in United States national parks using dragonfly larvae as biosentinels through a citizen-science framework: Environmental Science \& Technology, v. 54, no. 14, p. 8779-8790, https://doi.org/10.1021/acs.est.0c01255.

Ewert, J.W., Diefenbach, A.K., and Ramsey, D.W., 2018, 2018 update to the U.S. Geological Survey national volcanic threat assessment: U.S. Geological Survey Scientific Investigations Report 2018-5140, 40 p., https://doi.org/10.3133/sir20185140.

Ewert, J.W., Guffanti, M., and Murray, T.L., 2005, An assessment of volcanic threat and monitoring capabilities in the United States-Framework for a National Volcano Early Warning System: U.S. Geological Survey, Open-File Report 2005-1164, 62 p., https://doi.org/10.3133/ofr20051164.

Halofsky, J.E., Andrews-Key, S.A., Edwards, J.E., Johnston, M.H., Nelson, H.W., Peterson, D.L., Schmitt, K.M., Swanston, C.W., and Williamson, T.B., 2018, Adapting forest management to climate change-The state of science and applications in Canada and the United States: Forest Ecology and management, v. 421, p. 84-87, https://doi.org/10.1016/j.foreco.2018.02.037.

Hammarstrom, J.M., Dicken, C.L., Day, W.C., Hofstra, A.H., Drenth, B.J., Shah, A.K., McCafferty, A.E., Woodruff, L.G., Foley, N.K., Ponce, D.A., Frost, T.P., and Stillings, L.L., 2020, Focus areas for data acquisition for potential domestic resources of 11 critical minerals in the conterminous United States, Hawaii, and Puerto Rico-Aluminum, cobalt, graphite, lithium, niobium, platinum-group elements, rare earth elements, tantalum, tin, titanium, and tungsten, chap. B of U.S. Geological Survey, Focus areas for data acquisition for potential domestic sources of critical minerals: U.S. Geological Survey Open-File Report 2019-1023, 67 p., https://doi.org/10.3133/ofr20191023B.

Hyer, K.E., and Phillips, S.W., 2021, USGS Chesapeake Science Strategy 2021-2025: U.S. Geological Survey Fact Sheet 2021-3037, 6 p., https://doi.org/10.3133/fs20213037.

Integrated Rangeland Fire Management Strategy Actionable Science Plan Team, 2016, The integrated rangeland fire management strategy actionable science plan: Washington D.C., U.S. Department of the Interior, 128 p., https://www.fs.usda.gov/treesearch/pubs/53265.
Kovach, R.P., Dunham, J.B., Al-Chokhachy, R., Snyder, C.D., Letcher, B.H., Young, J.A., Beever, E.A., Pederson, G.T., Lynch, A.J., Hitt, N.P., Konrad, C.P., Jaeger, K.L.,

Rea, A.H., Sepulveda, A.J., Lambert, R.M., Stoker, J., Giersch, J.J., and Muhlfeld, C.C., 2019, An integrated framework for ecological drought across riverscapes of North America: BioScience, v. 69, no. 6, p. 418-431, https://doi.org/10.1093/biosci/biz040.

Jackson, S.T., 2011, Walking through time in the Lake Michigan dunes: Frontiers in Ecology and Environment, v. 9 , no. 9 , p. 526-527, https://doi.org/10.1890/15409295-9.9.526.

Jaeger, K.L., Burnett, J., Heaston, E.D., Wondzell, S.M., Chelgren, N., Dunham, J.B., Johnson, S., and Brown, M., 2020, FLOwPER user's guide-For collection of FLOw PERmanence field observations: U.S. Geological Survey Open-File Report 2020-1075, 40 p., https://doi.org/10.3133/ofr20201075.

Jaeger, K.L., Sando, R., McShane, R.R., Dunham, J.B., Hockman-Wert, D.P., Kaiser, K.E., Hafen, K., Risley, J.C., and Blasch, K.W., 2019, Probability of streamflow permanence model (PROSPER) - A spatially continuous model of annual streamflow permanence throughout the Pacific Northwest: Journal of Hydrology X, v. 2, 19 p., https://doi.org/10.1016/j.hydroa.2018.100005.

Martin, J.T., Pederson, G.T., Woodhouse, C.A., Cook, E.R., McCabe, G.J., Anchukaitis, K.J., Wise, E.K., Erger, P.J., Dolan, L.S., McGuire, M., Gangopadhyay, S., Chase, K.J., Littell, J.S., Gray, S.T., St. George, S., Friedman, J.M., Sauchyn, D.J., St. Jacques, J.-M., and King, J.C., 2020, Increased drought severity tracks warming in the United States' largest river basin: Proceedings of the National Academy of Sciences, v. 117, no. 21, p. 11328-11336, https://doi.org/10.1073/pnas.1916208117.

Miller, M.P., Clark, B.R., Eberts, S.M., Lambert, P.M., and Toccalino, P., 2020, Water priorities for the Nation-U.S. Geological Survey Integrated Water Availability Assessments: U.S. Geological Survey Fact Sheet 2020-3044, 2 p., https://doi.org/10.3133/fs20203044.

Moore, R.B., Johnston, C.M., Robinson, K.W., and Deacon, J.R., 2004, Estimation of total nitrogen and phosphorus in New England streams using spatially referenced regression models: U.S. Geological Survey Scientific Investigations Report 2004-55012, 42 p., https://doi.org/10.3133/sir20045012.

Mullaney, J.R., 2016, Nutrient, organic carbon, and chloride concentrations and loads in selected Long Island Sound tributaries-Four decades of change following the passage of the Federal Clean Water Act: U.S. Geological Survey Scientific Investigations Report 2015-5189, 47 p., https://doi.org/10.3133/sir20155189. 
Murphy, S.F., McCleskey, R.B., Martin, D.A., Holloway, J.M., and Writer, J.H., 2020, Wildfire-driven changes in hydrology mobilize arsenic and metals from legacy mine waste: The Science of the Total Environment, v. 743, 15 p., https://doi.org/10.1016/j.scitotenv.2020.140635.

Ostroff, A.C., Muhlfeld, C.C., Lambert, P.M., Booth, N.L., Carter, S.L., Stoker, J.M., and Focazio, M.J., 2017, USGS integrated drought science: U.S. Geological Survey Circular 1430, 24 p., https://doi.org/10.3133/cir1430.

Overpeck, J.T., and Udall, B., 2020, Climate Change and the acidification of North America: Proceedings of the National Academy of Sciences, v. 117, no. 22, p. 11856-11858, https://doi.org/10.1073/pnas.2006323117.

Pearson, J., 2020, Carp, climate, and water quality: Applying systems modeling to ecosystem recovery in Malheur Lake, Oregon-An abstract for the dissertation of J. Person: Oregon State University, https://ir.library.oregonstate.edu/ downloads $/ 8336 \mathrm{~h} 8146$ ? locale $=$ en .

Read, J.S., Jia, X., Willard, J., Appling, A.P., Zwart, J.A., Oliver, S.K., Karpatne, A., Hansen, G.J.A., Hanson, P.C., Watkins, W., Steinbach, M., and Kumar, V., 2019, Process-guided deep learning predictions of lake water temperature: Water Resources Research, v. 55, no. 11, p. 9173-9190, https://doi.org/10.1029/2019WR024922.

Schwarz, G.E., Hoos, A.B., Alexander, R.B., and Smith, R.A., 2006, The SPARROW surface water-quality model-Theory, applications and user documentation: U.S. Geological Survey Techniques and Methods 6-B3, 248 p., https://doi.org/10.3133/tm6B3.

Smith, D.B., Solano, F., Woodruff, L.G., Cannon, W.F., and Ellefsen, K.J., 2019, Geochemical and mineralogical maps, with interpretation, for soils of the conterminous United States: U.S. Geological Survey Scientific Investigations Report 2017-5118, https://doi.org/10.3133/sir20175118.
Sohl, T., Dornbier, J., Wika, S., and Robison, C., 2019, Remote sensing as the foundation for high-resolution United States landscape projections-The land change monitoring, assessment, and projection (LCMAP) initiative: Environmental Modelling and Software, v. 120, 17 p., https://doi.org/10.1016/j.envsoft.2019.104495.

Steblein, P.F., Loehman, R.A., Miller, M.P., Holomuzki, J.R., Soileau, S.C., Brooks, M.L., Drane-Maury, M., Hamilton, H.M., Kean, J.W., Keeley, J.E., Mason, R.R., Jr., McKerrow, A.J., Meldrum, J.R., Molder, E.B., Murphy, S.F., Peterson, B., Plumlee, G.S., Shinneman, D.J., van Mantgem, P.J., and York, A., 2021, U.S. Geological Survey wildland fire science strategic plan, 2021-26: U.S. Geological Survey Circular 1471, 30 p., https://doi.org/10.3133/cir1471.

U.S. Department of the Interior, Bureau of Land Management, ed., 2016, The Federal Land Policy and Management Act of 1976, as amended: U.S. Department of the Interior, Bureau of Land Management, Office of Public Affairs, Washington, DC. 106 p., https://www.blm.gov/sites/blm.gov/files/ AboutUs_LawsandRegs_FLPMA.pdf.

U.S. Geological Survey, 2021, U.S. Geological Survey 21st-century science strategy 2020-2030: U.S. Geological Survey Circular 1476, 20 p., https://doi.org/10.3133/cir1476.

Webster, J.P., Kane, T.J., Obrist, D., Ryan, J.N., and Aiken, G.R., 2016, Estimating mercury emissions resulting from wildfire in forests of the Western United States: The Science of the Total Environment, v. 568, p. 578-586, https://doi.org/10.1016/j.scitotenv.2016.01.166.

Zell, W.O., and Sanford, W.E., 2020, Calibrated simulation of the long-term average surficial groundwater system and derived spatial distributions of its characteristics for the contiguous United States: Water Resources Research, v. 56, no. 8, 16 p., https://doi.org/10.1029/2019WR026724. 


\section{Appendix 6. Use Case Decision Support Statements}

Members of the Use Case Development team reviewed each of the Use Case summaries with a goal of extracting and summarizing the decision-support focus. The intent was to summarize the Use Case by filling in the elements in brackets in the following "Decision Support Statements": This Use Case will produce [a product or a result] that [will/ can] be used by [decision-maker(s)] to support [one or more decisions].

Most Use Cases could be summarized this way, although the statement template was modified in a few instances. These summaries are included later in the text, organized by region, and the full Use Case descriptions as submitted by the regions are included in appendix 5. These summary statements were not provided by the original authors of the Use Cases, nor have they been reviewed by those authors. They represent, instead, a "third party" summary of information presented in those write-ups. In any future outreach efforts to solicit this kind of information from scientists across the Bureau, it could be useful to ask the submitters to include their own summary table of this information.

\section{Southeast Region-Use Case Decision Support Statements}

\section{Use Case-Mississippi Alluvial Plain Water Availability Study}

This activity results in: Co-design and co-production of monitoring data and predictive models of water availability, water quality, ecological habitats, and socio-economic assessments of water use under various stakeholder defined scenarios.

Which can/will be used by: A wide array of federal (United States Army Corps of Engineers [USACE], U.S. Dept. of Agriculture), state (Arkansas, Louisiana, Mississippi, and Missouri) agencies and universities, and nongovernmental organization (NGO; The Nature Conservancy, Delta Wildlife) partners.

To support: Decisions to secure future water resources (for example, improved decisions about investment in alternative water-supply options).

\section{Use Case-Alabama Barrier Island Restoration Assessment}

This project produced ${ }^{1}$ : An evaluation of options for the restoration of Dauphin Island in Alabama to restore vital habitat for species affected by the 2010 Deepwater Horizon spill and increase island sustainability against sea level rise and coastal storms.

Which were used by: The State of Alabama.

To support: Targeting of over $\$ 150$ million worth of restoration measures for implementation.

\section{Midcontinent Region-Use Case Decision Support Statements}

\section{Use Case- Earth Monitoring, Analysis, and Prediction (EarthMAP) as a strategic, systematic interface between U.S. Geological Survey and the National Park Service Resource Stewardship Strategies (RSS) ${ }^{2}$}

Will produce: A system of data collection, analysis and prediction relevant to the elements of Park-specific RSS, such as current and predicted landscape and species conditions (for example, species diversity, biotic communities and their productivity, vulnerability). Initial activities would focus on more automated delivery of information on status of species and physical processes in the Indiana Dunes National Park (IDNP) through time.

Which can/will be used by: Resource managers of National Parks [the manager of the Indiana Dunes National Park].

To support: Evaluation, selection, and monitoring of actions to implement Resource Stewardship Strategies [unspecified].

\footnotetext{
${ }^{1}$ This Use Case described a completed project, so the template was modified slightly to reflect this as an activity that was completed and used.

${ }^{2}$ This Use Case described both a general need and a specific set of initial steps; the summary includes both, with the specifics shown in brackets.
} 
Use Case-U.S. Geological Survey Integrated Ecohydrology Science: A New Path to Advance Water Science, Ecosystem Science, and Earth-System Prediction

Will produce: Daily current and predicted streamflow discharge, stream-network connectivity, wetland extent, relative concentrations of ecologically relevant contaminants, and probabilistic risk of hazard (for example, flood or drought), presented at multiple relevant ecohydrologic scales (for example, reach, basin, region) for all locations in the Midcontinent Region, through an interactive Web-based interface.

Which can/will be used by: Land mangers (for example, foresters, agriculturists) and the public.

To support: Their decisions about how to mitigate and adapt to the impacts that changes in water supply/quality and ecosystem services that affect their work, livelihood and recreation.

\section{Use Case-Harmful Algal Bloom Monitoring and Prediction (HABMAP)}

Will produce: Predictions of the location and timing of high toxicity harmful algal blooms (HABs) and nuisance washup events, using real-time information.

Which can/will be used by: Land managers and managers of waters for municipal, industrial, and recreational purposes.

To support: Decisions to mitigate or minimize the environmental health and economic impacts of HABs (for example, by switching water sources, posting warnings, and so forth).

\section{Use Case-Legacy Sources of Nutrients and Implications for Management Strategies}

Will produce: Full descriptions of current and legacy pools of nutrients within watersheds (including age, chemical profiles, nutrient gradients and stoichiometry, areal distributions, and transport processes) and predictions of how best management practices for nutrient remediation and management will perform.

Which can/will be used by: Resource managers working to improve water quality across the Nation.

To support: Decisions about where, when, and how to implement the most effective best management practices considering both legacy and ongoing nutrient sources.

\section{Use Case-Expanding Environmental DNA Tracking of Pathogens and Invasive Species using the NowCast Framework}

Will produce: Updated NowCast products that include environmental DNA (eDNA) sampling results for aquatic species that indicate a threat to public health and aquatic communities (such as, E. coli, avian botulism, grass carp, Dreissena spp. mussels, lampreys, milfoils, and round goby), presented through map-based tools.

Which can/will be used by: Federal, state, and local resource managers.

To support: Decisions about public health advisories, warning, or closures to protect human health and sensitive aquatic native/endangered species from waterborne pathogens and nonindigenous species and where nonindigenous species control should be undertaken.

\section{Northeast Region-Use Case Decision Support Statements}

\section{Use Case-An Integrated Framework for Assessing Bird Populations to Better Understand Drivers of Population Change and Project Future Outcomes}

Will produce: An integrated modeling framework for assessing and visualizing national and regional bird population abundance and trends using both the North American Breeding Bird Survey (BSSS and eBird data, including projections of changes in population size and distributions based on different environmental and management scenarios, and specifically, will (1) increase the geographic and operational scope of recently developed integrated population models, (2) improve the precision of bird population estimates, and (3) provide forecasts at multiple temporal scales relevant to the Department of the Interior and partners.

Which can/will be used by: External partners engaged in bird management and conservation (for example, U.S. Fish and Wildlife Service, U.S. state agencies, Canadian Wildlife Service, Mexican National Commission for the Knowledge and Use of Biodiversity).

To support: Annual regulatory decisions on management of North American bird species and understanding of changes in bird populations over longer time scales in response to climate and land-use change. ${ }^{3}$

\footnotetext{
${ }^{3}$ Several Use Case descriptions mentioned a primary goal of supporting increased knowledge, rather than supporting decisions; this Use Case mentioned both.
} 
Use Case-Integrated Monitoring, Analysis, and Modeling for Enhanced Predictive Capability of Nutrient Loads from the Connecticut River Basin to Long Island Sound

Will produce: An integrated monitoring, analysis, and modeling framework to enhance predictive capability of nutrient loading from the Connecticut River Basin to Long Island Sound, incorporating dynamic (seasonal) transport processes and nonpoint-source loading of nutrients from groundwater into the modeling and analysis framework.

Which can/will be used by: International (Canada), national, state, and local resource managers.

To support: Decisions about how, where, and when to conduct mitigation measures aimed at improving water quality throughout the watershed and decisions about how to manage the impacts of nutrient loading to freshwater and marine wildlife, fisheries, vegetation, and dynamics of harmful algal blooms.

\section{Use Case-Sediment-Observation, Assessment, Prediction, and Operationalizing of a Regional Analysis of Sediment and Co-contaminate Flux and Sediment Sources in the Delaware River Basin}

Will produce: Improved estimates and predictions of sediment fluxes, sources, and co-contaminant flux in the Delaware River Basin and identification of specific reaches with high contributions to sediment problems (potentially expandable to other geographies).

Which can/will be used by: Local, state, regional, federal, academic and NGO stakeholders, including a broad array of resource managers from drinking water purveyors, to state environmental agencies, to industrial water users, as well as wildlife managers and sportsman.

To support: Decisions related to management of sediment population, associated co-contaminates, and decisions about restoration of aquatic ecosystems impaired by excess sediment.

\section{Use Case-Integrated Multidisciplinary Science to Inform Habitat Conservation and Management Efforts for Fish and Wildlife in the Delaware Watershed}

Will produce: Integrated ecosystem science related to recreational and commercially important fish species, waterbirds, benthic macroinvertebrates, other aquatic and semi-aquatic species, pollinators, and their habitats in the Delaware River Basin.
Which can/will be used by: The Delaware River Basin Commission, Delaware River Basin Restoration Program, and other stakeholders.

To support: Decisions on the conservation, management, and restoration of these resources/habitats to sustain the living resources in the ecosystem.

\section{Use Case-Improving National Fish Habitat Assessments using the National Hydrography Dataset Plus High Resolution: An EarthMAP Pilot Effort in the Delaware River Basin}

Will produce: Fine scale $(1: 24,000)$ hydrologic framework (National Hydrography Dataset [NHD] Plus High Resolution [HR]) and assessments of the status of fish habitats in the United States [starting with an application in the Delaware River Basin].

Which can/will be used by: Federal and state agencies and nongovernmental organizations (NGOs).

To support: The design and implementation of habitat assessments and associated conservation, mitigation, and restoration activities.

\section{Use Case-Optimization of Mineral Resource Data: Mine Wastes, Resource Assessments, and Focus Areas}

Will produce: Geospatial information on mineral resources, including where minerals have been mined in the past, the location of wastes associated with their production and processing, and prospective areas for future mineral production.

Which can/will be used by: unidentified decision makers.

To support: Decisions about where mineral resources should be extracted to (1) maximize economic efficiency, sustainability, and security of supply and (2) minimize hazards to human health and the environment and risks of supply disruption.

\section{Use Case-Collaborative Science to Enhance Resilience in Coastal Cities, Towns, and Rural Landscapes: The Urban Coastal Resilience Initiative (UCRI)}

Will produce: Predictive science products about coastal resilience to change linked to socio-economic information (starting with a compound flooding model applied to New York City and its boroughs). 
Which can/will be used by: Various natural resource and infrastructure managers, including municipal managers and planners, NGO environmental and health organizations, flood- or erosion-prone communities, transportation, health and human service managers (initially the New York City Department of Environmental Protection [NYC DEP] and Mayor's Office of Resiliency [MOR]).

To support: Development of coherent, long-term, location-specific resiliency plans for coastal urban areas subject to environmental disturbance (for example, best practice solutions for infrastructure, vulnerability assessment and response planning, flood protection). Specific decision support focus of NYC DEP and MOR to be developed.

\section{Alaska Region-Use Case Decision Support Statements}

\section{Use Case-Enhanced Delivery of Integrated Ecosystem Model Output}

Will produce: Predictions and visualization of vegetation response and associated wildfire risk under different climate change scenarios in sub-arctic and boreal landscapes in Alaska, as an enhancement to the existing Alaska Integrated Ecosystem Model.

Which can/will be used by: Bureau of Land Management (BLM), Alaska Fire Service, State of Alaska Division of Forestry, National Park Service, U.S. Department of Agriculture (USDA) Forest Service, regional and local planning departments, and Alaska Native communities.

To support: More informed assessments of future fire hazards, reduction of wildfire risk, enhanced ecological resilience, and improved wildlife habitat management, ultimately to support fire risk management decisions. ${ }^{4}$

\section{Use Case-Wildlife Tracking Portal to Improve Data Documentation, Delivery, and Analysis}

Will produce: An extension of the ASC Wildlife Tracking Data Collection to improve efficiency in wildlife satellite tag data management and metadata development and expanded delivery of tracking data (which will in turn support additional analyses, such as habitat use, distribution, and timing of migration). ${ }^{5}$

Which can/will be used by: U.S. Fish and Wildlife Service, Bureau of Land Management, Bureau of Ocean Energy Management, National Park Service, U.S. Forest Service, and state and local agencies.

To support: Land and resource management decisions informed by biogeographic analysis of sites such as patterns of habitat use and identification of repeated geographic locations important for monitored species and populations.

\section{Use Case-Machine Learning Analysis of Near Real-time Imagery of U.S. Volcanoes}

Will produce: Continuous automated assessment of incoming real-time images of monitored volcanoes to detect, classify, and notify sudden, remarkable changes, using machine learning techniques.

Which can/will be used by: The USGS Volcano Science Center and potentially other entities who monitor volcanoes.

To support: Improved timeliness and accuracy of volcano forecasts and warnings.

\section{Use Case-Customized Arctic Map Products for State, Federal, and International Use}

Will produce: Five new Arctic Research Policy Act Boundary map products, including marine and terrestrial domains that can be accessed using a new, interactive web application.

Which can/will be used by: USGS scientists, U.S. State Department, National Oceanic and Atmospheric Administration (NOAA), U.S. Coast Guard, and State of Alaska Department of Natural Resources.

To support: updated and integrated situational awareness and relevant modeling information for national and international stakeholders who are concerned about the features of a rapidly changing Arctic.

\footnotetext{
${ }^{4}$ Several Use Cases, including this one, indicated a primary goal of increased knowledge and more baseline understanding, that would then be analyzed further or combined with other information to support a variety of unspecified decisions.
} 


\section{Use Case-Building an Integrated Coastal Hazard Assessment and Mitigation Strategy with Bering Sea Communities of Alaska}

Will produce: Coastal storm inundation risk assessment maps (combining USGS and partner data).

Which can/will be used by: Local planners in Bering Sea communities as well as federal, state, tribal and local planners, resource managers, and emergency responders.

To support: Adaptation and resilience planning efforts.

\section{Southwest Region-Use Case Decision Support Statements}

Use Case-Enhancing Situational Awareness and Forecast Models for Post-Wildfire Runoff, Recharge, Water Quality, and Sedimentation.

Will produce: Monitoring networks and associated modeling to be rapidly deployed in burn areas to provide real-time streamflow data, flood inundation mapping, debris flow alerts, and short- and long-term forecasts of streamflow and sediment fluxes.

Which can/will be used by: Bureau of Reclamation, U.S. Army Corps of Engineers, California Department of Water Resources (CA DWR), local utilities (PG\&E), Burned Area Emergency Response (BAER) teams, and county water management agencies.

To support: Near-term post-fire decisions regarding prioritization of erosion-control measures to protect water resources and, over longer time scales, information about how landscapes recover from fire in order to make decisions to protect water and ecosystem resources.

\section{Use Case-Data and modeling platform to support forecasting California Water Project Effects}

Will produce: A set of peer-reviewed, public domain environmental models linking surface-water flows, sediment dynamics, and physical geography to the distribution and quality of habitats and ecosystem services in the Central Valley of California, presented in an integrated in a data platform.

Which can/will be used by: California water management entities (including the CA Water Project and individual operators within that system).

To support: Both long-term planning decisions and near-term operational decisions about water allocations.

\section{Use Case-A Drought Data Explorer for the Colorado River Basin: Integrated and Dynamic Web-based Delivery of Actionable Information}

Will produce: Interactive, web-based dynamic delivery of integrated data, models, and tools based on from the collective dryland and drought based research of the Terrestrial Drylands Ecology Branch of the Southwest Biological Science Center.

Which can/will be used by: Land managers from a variety of federal and state agencies, private entities, and academic partners.

To support: Decisions about how to mitigate, adapt, and prepare for drought-induced changes to the landscape.

\section{Use Case-Improving Daily Modeled Estimates of High Elevation Snowpack to Boost Runoff Forecast Accuracy in Colorado Basin Watersheds}

Will produce: Improved estimates of snow-water-equivalent (SWE) in high elevation areas using remote sensing and improved modeling (which in turn can be used to improve daily/weekly/monthly predictions of snowmelt runoff).

Which can/will be used by: Water resource managers, reservoir operations, flood forecasters, and hydropower operations.

To support: Decisions for optimizing reservoir operations, water deliveries, hydropower production, and water delivery volume and timing.

\section{Use Case-Predicting Water Quality and Environmental Health in the Rivers of the Colorado River Basin Affected by Wildfire}

Will produce: An empirical relation between geology, fire intensity, geochemical modeling, and dissolved oxygen (DO) concentrations in streams (supporting future development of a predictive model of post-wildfire stream quality).

Which can/will be used by: Other modelers and models (for example, of water-quality, geochemistry, fire science, geology, soils, precipitation, stream flow, and ecology, and ultimately unspecified decision-makers and stakeholders).

To support: Predictions of how future wildfires could affect DO concentrations in downstream aquatic habitats and water resources (to be used as part of a more comprehensive model to support decisions about fish or habitat conservation and human health protection related to water quality after wildfires). 


\section{Rocky Mountain Region-Use Case Decision Support Statements}

\section{Use Case-Integrated Science to Enhance Prediction of Post-fire Hazards, Risk Assessments, and Decision Making}

Will produce: Integrated predictive models for fire occurrence and severity, impacts of fire on social and ecological resources, potential for post-fire risks, such as debris flows and changes in water quality and quantity, and the effectiveness of post-fire recovery actions.

Which can/will be used by: Wildland fire and emergency management communities, represented by the Wildland Fire Leadership Council.

To support: Decisions about targeting pre- and during-fire protection and fire-fighting resources and decisions about post-fire mitigation and recovery.

\section{Use Case-Daily and Seasonal Forecasts of Groundwater and Surface Water Availability and Their Interactions in the Upper Colorado River Basin}

Will produce: A model of integrated hydrology of the upper Colorado River Basin (CRB) to produce continuously updated daily, monthly, and seasonal forecasts of water availability at a 1-square kilometer $\left(\mathrm{km}^{2}\right)$ scale that result from improved integration of groundwater, surface water, and atmospheric interactions.

Which can/will be used by: Federal, state, and local water resource managers.

To support: Meeting water delivery requirements and managing ecosystems while planning for potential changes in water use and future water supplies in changing climate conditions, including periods of excess water and droughts.

\section{Use Case-Integrating Environmental and Economic Data and Modeling to Develop a Multi-objective Resource Planning Tool}

Will produce: An interactive decision support tool that will integrate hydrologic, ecosystems, and economic data and models with scenario analyses (and specifically, starting by linking streamflow and water-quality models in the upper Colorado River Basin).
Which can/will be used by: State, tribal and federal entities with resource management responsibilities (and specifically, Upper Colorado River Basin [UCRB] entities responsible for implementing the Drought Contingency Plan).

To support: Various decisions involving reservoir operations, water allocations, habitat and ecologic flows where balancing multiple and competing management objectives is required.

\section{Use Case-Fire Impact on Water Quality and Ecosystem Function in the Rocky Mountain Region}

Will produce: A spatial risk analysis of post-fire impacts on surface-water quality and ecotoxicology related to trace metals released from soil during wildfires, identifying watersheds that are more sensitive to trace-metal mobilization following wildfire.

Which can/will be used by: USDA, United States Forest Service (USFS), National Park Service (NPS), USFWS, tribal fishery programs, state fish and wildlife agencies, and municipal water suppliers.

To support: Decisions on prioritization of monitoring sites and developing attainable post-fire monitoring goals for mitigation and risk reduction measures; further decisions to be explored with the stakeholder identified for this Use Case.

\section{Use Case-Energy Resource Development Potential and Tradeoffs, to Support Integrated Analysis for "All of the Earlier Mentioned" Energy Strategies}

Will produce: A decision support tool to estimate future energy development potential, including co-located resources and associated tradeoffs and locations where energy development is more likely based on a set of predictor variables; information on the associated social-ecological system(s), water source and use areas, key wildlife habitat areas, protected areas, transmission infrastructure, and established socio-economic uses, such as recreation, aesthetic enjoyment, water-quality regulation, and others.

Which can/will be used by: State and federal land and wildlife managers and individuals and groups with varied interests in resource use, such as recreational interests, private property owners, and wildlife advocates.

To support: Decisions and planning efforts on the future impacts of fossil fuel and renewable energy development on land, water, and socio-ecological resources. 


\section{Use Case-Predicting Future Water-Quality Changes in Mineralized Headwater Catchments in the Upper Colorado River Basin}

Will produce: An empirical geographic information system (GIS)-based modeling tool predicting the likelihood of future water-quality degradation in mineralized mountain headwater catchments within the UCRB because of climate warming.

Which can/will be used by: Local, state and federal agencies, such as municipal water resources managers, USFWS, and U.S. Environmental Protection Agency (EPA).

To support: Management of surface-water resources, fish populations, and abandoned mine site cleanup efforts.

\section{Use Case-Resource Assessment Platform}

Will produce: A centralized platform synthesizing and integrating existing data and analytical tools providing information on water resources, climate, land cover and land use, geology, mineral and energy resources, species, and ecosystems in the Colorado River Basin.

Which can/will be used by: A wide range of federal, tribal, and state resource managers working on the Colorado River Basin.

To support: The evaluation of environmental impacts of federal agency actions under the National Environmental Policy Act (NEPA) and decisions about:

Recovery and management of threatened and endangered species, and

federal, tribal, and state management of land, forestry, energy, and other resources.

\section{Northwest and Pacific Islands Region-Use Case Decision Support Statements}

\section{Use Case-Thunder and Lightning-Social, Ecological, and Hydrologic Assessment of Drought in the Donner und Blitzen River Watershed}

Will produce: A "visible riverscape" that allows stakeholders to view spatially and temporally variable meteorological, hydrologic, ecological conditions and current social landscapes (initially in the Donner und Blitzen watershed in Washington State).

Which can/will be used by: Managers with responsibilities to address drought, including the U.S. Forest Service, Bureau of Land Management, and National Park Service.
To support: Decisions to reduce the increasing threat posed by drought on social, ecological, and hydrological systems through, for example, long-term drought adaptation planning and seasonal to annual changes in water use for agriculture, livestock grazing, and fish and wildlife management.

\section{Use Case-Geography of change-Synthesizing Multiple Dimensions of Anthropogenic Global Change With Indicators of Ecological and Hydrological Conditions}

Will produce: A synthesis of existing data products to describe the intensity of anthropogenic change and predict its implications for natural communities and ecological services (starting with reach-to-watershed assessments of ecohydrological conditions in the Pacific Northwest).

Which can/will be used by: An unspecified range of local municipalities to federal agencies (for example, USFS, BLM).

To support: Identification of multi-dimensional refugia and inform prioritization decisions for conservation and climate mitigation.

\section{Use Case-From Water to Wildlife: Linking Water Timing and Availability to Subalpine and Alpine Vegetation and Wildlife Population}

Will produce: Prediction of the responses of sub-alpine and alpine vegetation and wildlife distributions to changes in water quality, availability, and seasonal timing, with an initial focus on the National Park Service units in Washington and Montana.

Which can/will be used by: Department of the Interior (DOI) partners, federal, tribal, and state agencies as well as adjacent land and property owners; NPS managers of Washington (WA) and Montana (MT) units.

To support: Decisions regarding the conservation, restoration, and maintenance of key wildlife and plant populations in sub-alpine and alpine environments.

\section{Use Case-Surface-Water Index of Permanence for Ecohydrology (SWIPE)}

Will produce: Risk-based interactive mapping tool for predicting surface-water permanence, stream-network connectivity, and invasive species and pathogen spread at multiple spatial and temporal scales and future climates.

Which can/will be used by: Land and water managers.

To support: Management of public lands and water resources, including management of water availability and quality, water rights, land use, invasive species and pathogens, and restoring connectivity among aquatic systems. 


\section{Use Case-Sustaining the Western Range: Actionable Science for Effective Management of the Sagebrush Biome}

Will produce: Data delivery and visualization platforms that integrate and make available past work of the Sage Working Group, spanning multiple western states.

Which can/will be used by: BLM, USFS, state Wildlife Agencies, local agencies, and NGOs who are part of the Sage Working Group.

To support: Land-use decisions affecting the sage biome, including conservation design and delivery, monitoring, and adaptive management across diverse and broad jurisdictional boundaries. 

For more information concerning the research in this report, contact the

Director, U.S. Geological Survey

12201 Sunrise Valley Drive, Reston, VA 20192,

Reston, VA 20192

https://www.usgs.gov/

Publishing support provided by the U.S. Geological Survey Science Publishing Network, Sacramento Publishing Service Center 


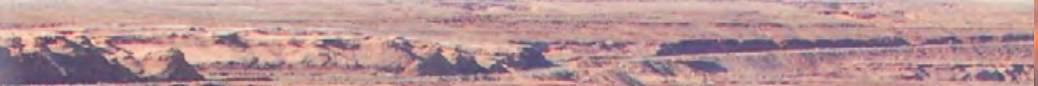

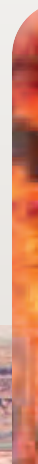

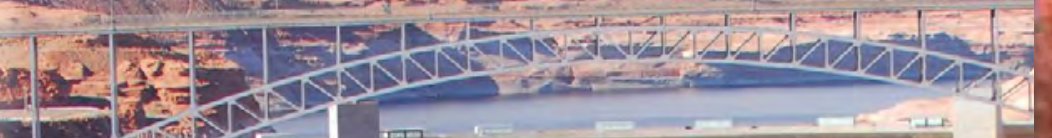

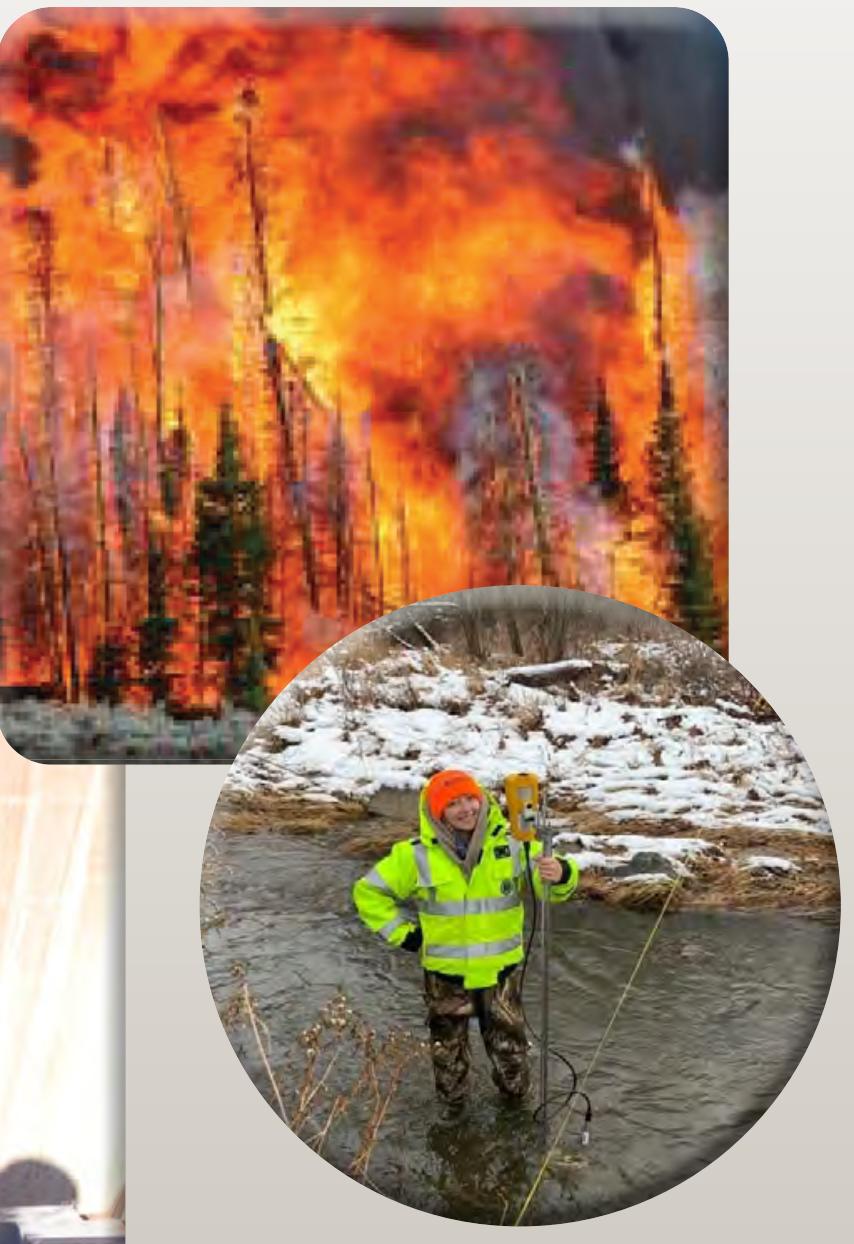
inis
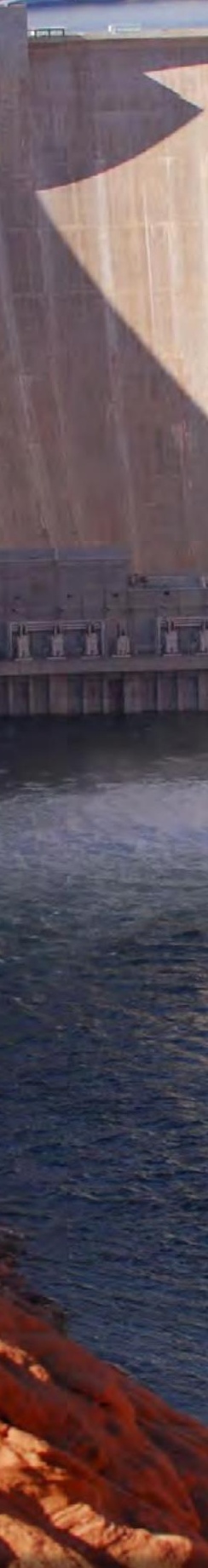